\title{
ELECTROWETTING AS A TOOL FOR TWO PHASE FLOW MICROFLUIDIC OPERATIONS
}

Arjen Michiel Pit 
Samenstelling promotiecommissie:

$\begin{array}{rlll}\text { Prof. Dr. } & \text { J.W.M. Hilgenkamp } & \text { (voorzitter) } & \text { Universiteit Twente } \\ \text { Prof. Dr. } & \text { F. Mugele } & \text { (promotor) } & \text { Universiteit Twente } \\ \text { Dr. } & \text { M.H.G. Duits } & \text { (co-promotor) } & \text { Universiteit Twente } \\ \text { Prof. Dr. } & \text { L.W.M.M. Terstappen } & \text { (opponent) } & \text { Universiteit Twente } \\ \text { Prof. Dr. } & \text { M.M.A.E. Claessens } & \text { (opponent) } & \text { Universiteit Twente } \\ \text { Prof. Dr. } & \text { J.C.T. Eijkel } & \text { (opponent) } & \text { Universiteit Twente } \\ \text { Prof. Dr. } & \text { J.M.J. den Toonder } & \text { (opponent) } & \text { Technische Uni. Eindhoven } \\ \text { Prof. Dr. } & \text { P.R. Onck } & \text { (opponent) } & \text { Rijksuniversiteit Groningen }\end{array}$

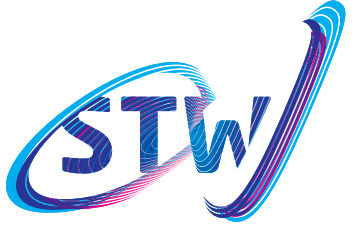

connecting innovators

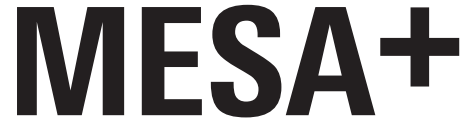

INSTITUTE FOR NANOTECHNOLOGY

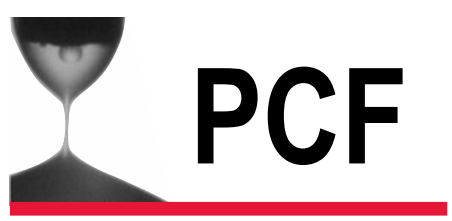

PHYSICS OF COMPLEX FLUIDS

The work in this thesis was carried out at the Physics of Complex Fluids group at the University of Twente. This research is supported by the Dutch Technology Foundation STW, which is part of the Netherlands Organization for Scientific Research (NWO), and which is partly funded by the Ministry of Economic Affairs.

Nederlandse titel:

Gebruik van electrowetting voor druppel manipulaties in twee-fasen microfluidica

Publisher:

Arjen Pit, Physics of Complex Fluids, University of Twente

P.O. Box 217, 7500AE Enschede, The Netherlands

www.utwente.nl/en/tnw/pcf/

Print: Proefschriftmaken.nl

(C) Arjen Pit, Enschede, The Netherlands, 2017

No part of this work may be reproduced by print photocopy of any other means without permission in writing from the publisher.

ISBN: 978-90-365-4431-3

DOI: $10.3990 / 1.9789036544313$ 


\title{
ELECTROWETTING AS A TOOL FOR TWO PHASE FLOW MICROFLUIDIC OPERATIONS
}

\section{PROEFSCHRIFT}

\author{
ter verkrijging van \\ de graad van doctor aan de Universiteit Twente, \\ op gezag van de rector magnificus, \\ Prof. dr. T.T.M. Palstra \\ volgens besluit van het College voor Promoties, \\ in het openbaar te verdedigen \\ op donderdag 23 november 2017 om 12:45 uur
}

door

\section{Arjen Michiel Pit}

Geboren op 18 april 1983

te Smallingerland 
Dit proefschrift is goedgekeurd door de promotoren:

Prof. Dr. F. Mugele

Dr. M.H.G. Duits 


\section{Contents}

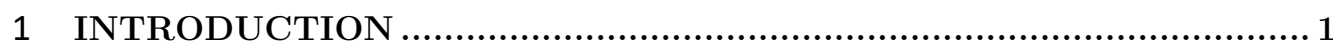

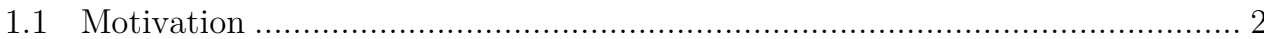

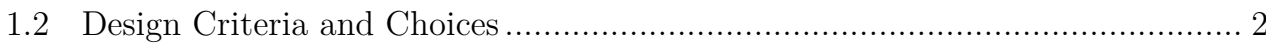

1.3 Manipulation of droplets using electrowetting ............................................. 3

1.3.1 Electrowetting Principle............................................................ 3

1.3.2 The electrowetting equation............................................................ 4

1.3.3 Applications of electrowetting ..................................................... 5

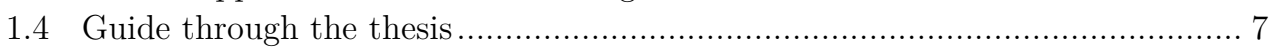

Appendix 1A: Derivation of the electrowetting equation...................................... 9

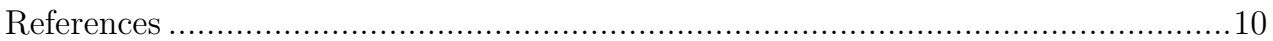

2 REVIEW: DROPLET MANIPULATIONS IN TWO PHASE FLOW

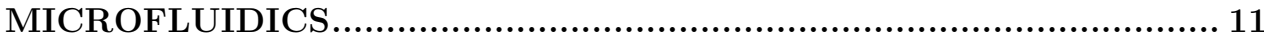

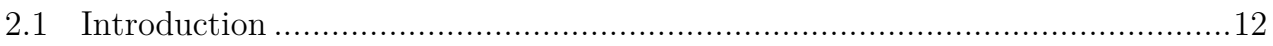

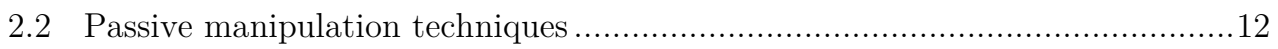

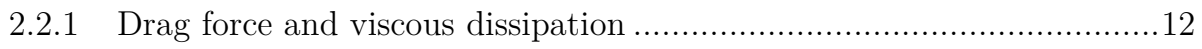

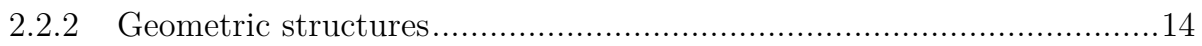

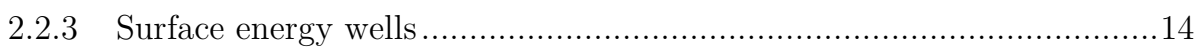

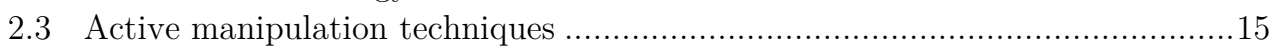

2.3.1 Pneumatic membrane (Quake) valves.............................................15

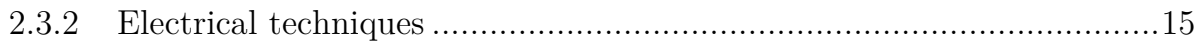

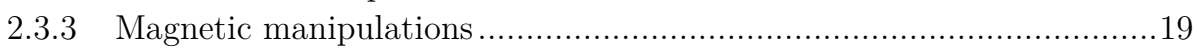

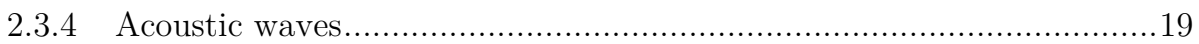

2.3.5 Optical manipulation techniques....................................................22

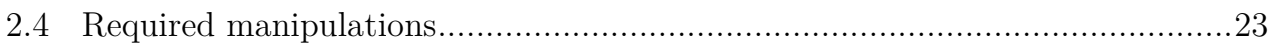

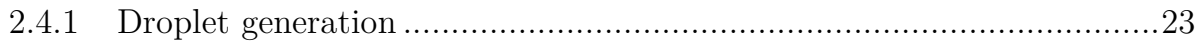

2.4.2 Droplet transport / guiding / steering ……....................................26

2.4.3 Droplet trapping and release .......................................................28

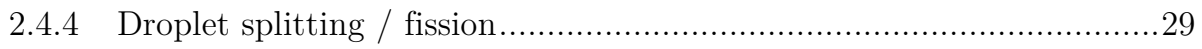

2.4.5 Droplet merging / coalescence / fusion ................................................ 31

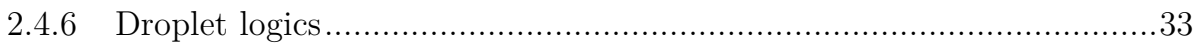

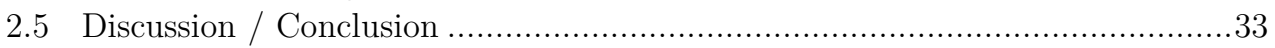

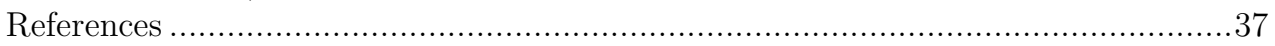

3 ELECTROSTATIC POTENTIAL WELLS ........................................... 43

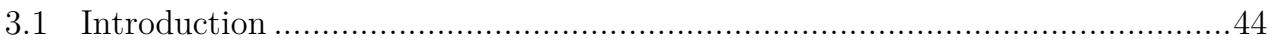

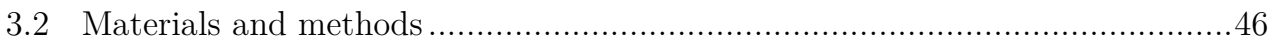

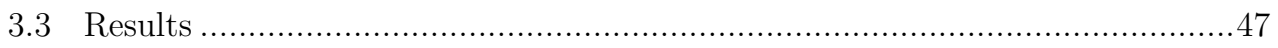

3.3.1 Principles of on-demand trapping and release ..................................4

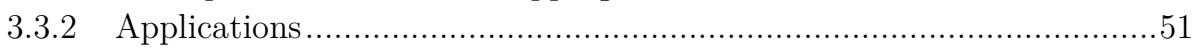

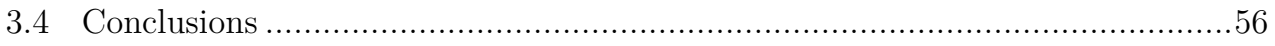

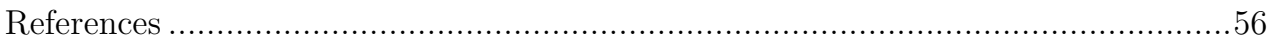




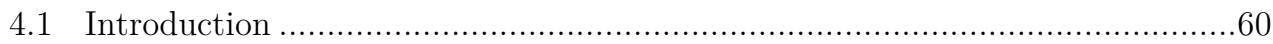

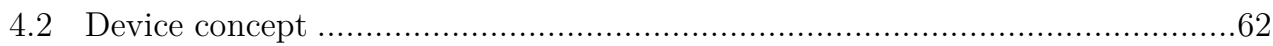

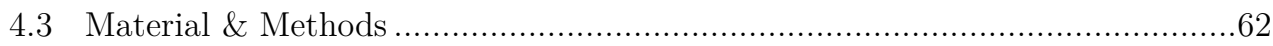

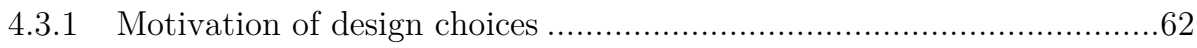

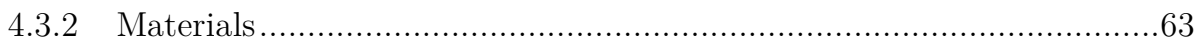

4.3.3 Electrowetting actuation surface (fabrication) ……………................63

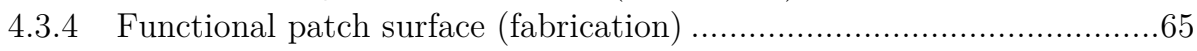

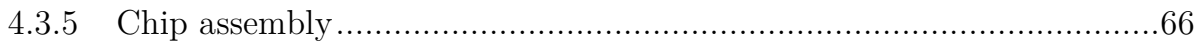

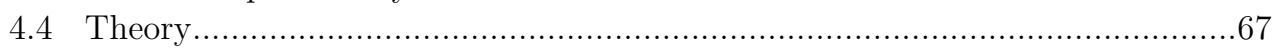

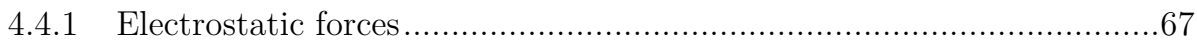

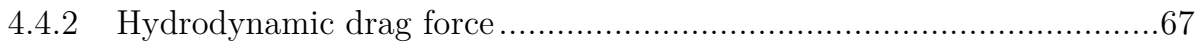

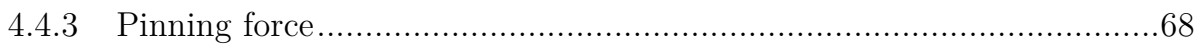

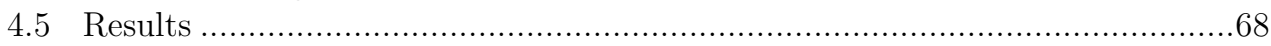

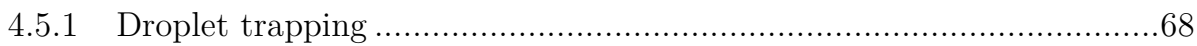

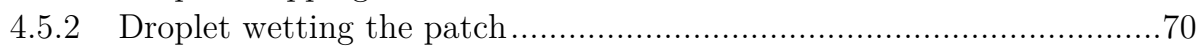

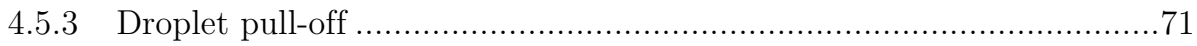

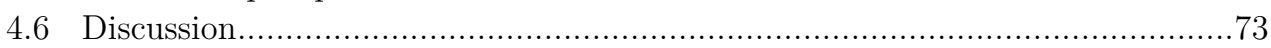

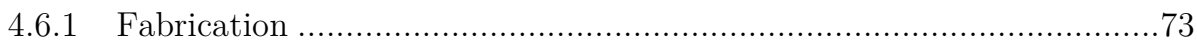

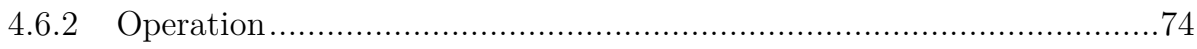

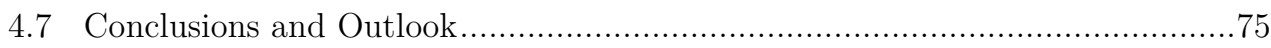

Appendix 4A: cleanroom masks for chip fabrication..........................................76

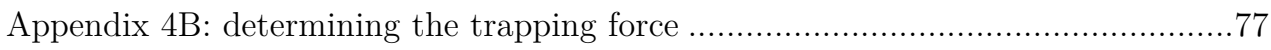

Appendix 4C: CA measurements for Cytop and MUA/gold..................................77

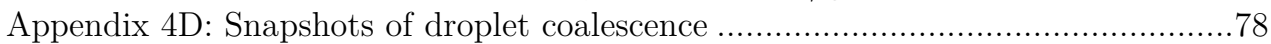

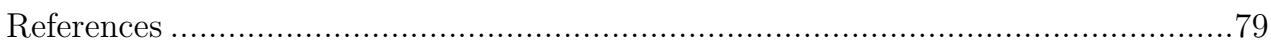

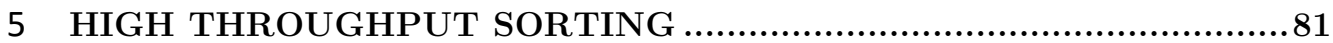

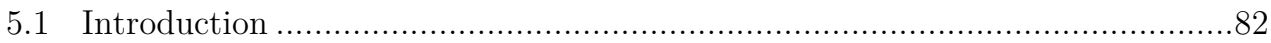

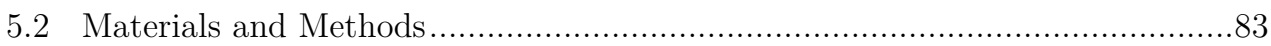

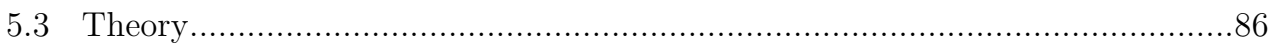

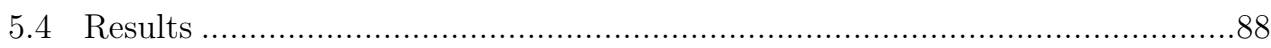

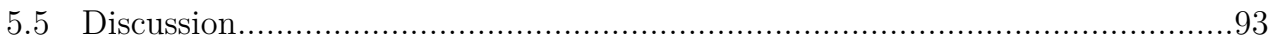

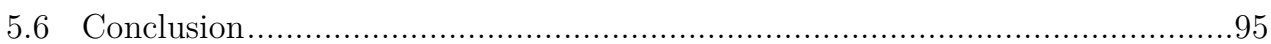

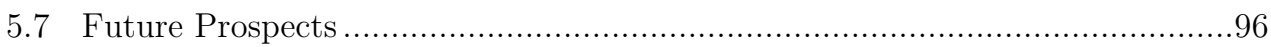

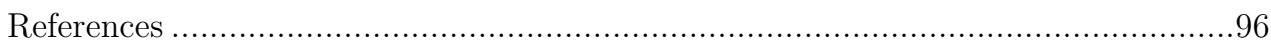

6 ELECTROWETTING IN THE DIELECTRIC REGIME ...................99

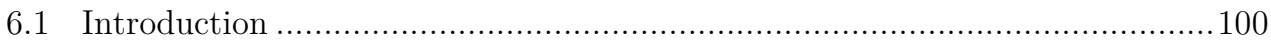

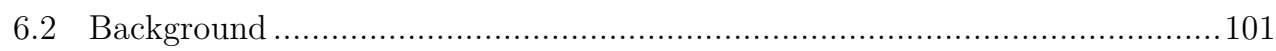

6.2.1 Frequency dependent contact angle …….................................... 101

6.2.2 Sorting efficiency for conductive and dielectric droplets...................102

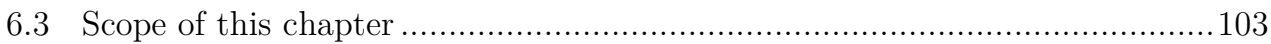

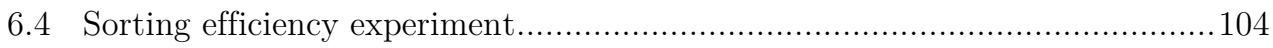




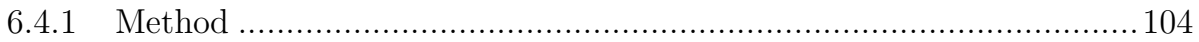

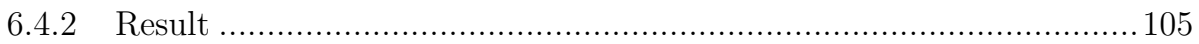

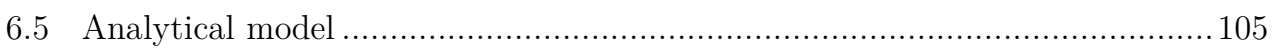

6.5.1 Lumped Element Model ............................................................ 106

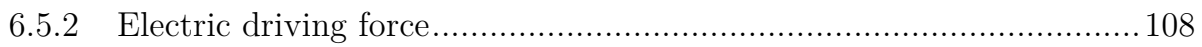

6.5.3 Results from LEM............................................................... 109

6.6 Numerical model ....................................................................... 111

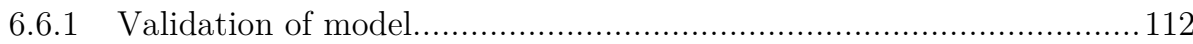

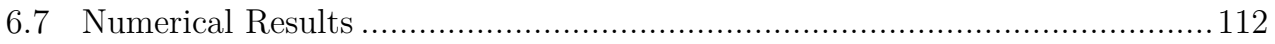

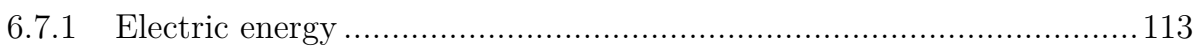

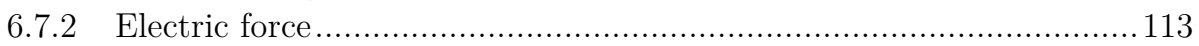

6.7.3 Normalization of electric energy .......................................... 115

6.7.4 Normalization of electric force ............................................ 115

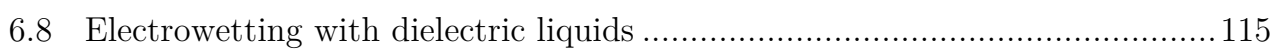

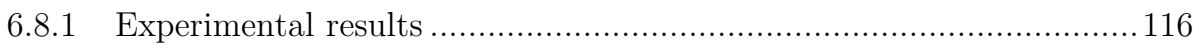

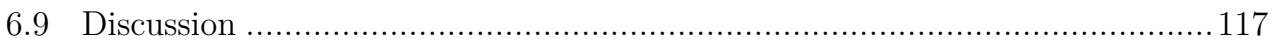

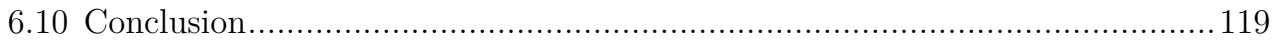

Appendix 6A: The lumped element model ................................................. 120

6A1 Describing the parallel plate system........................................ 120

6A2 The sum of electric field energy equals the total electric energy ........ 121

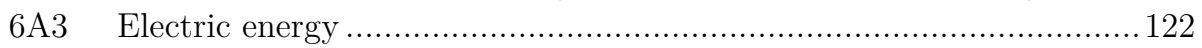

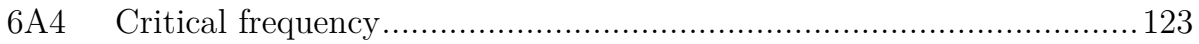

Appendix 6B: Electric potential and electric field lines .................................. 124

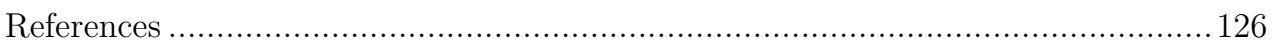

7 CONCLUSIONS AND OUTLOOK ......................................... 129

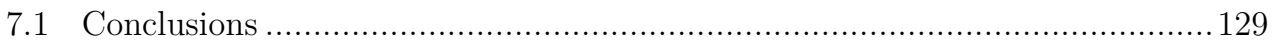

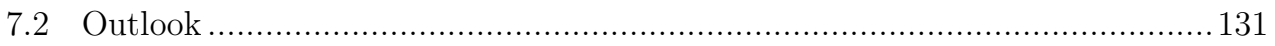

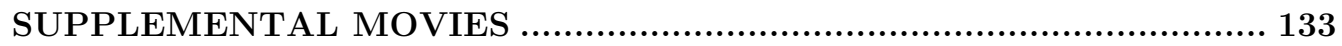

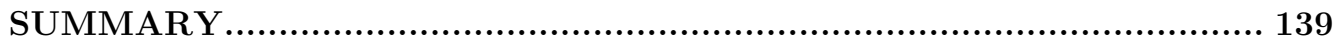

SAMENVATTING............................................................. 141

LIST OF PUBLICATIONS ................................................. 143

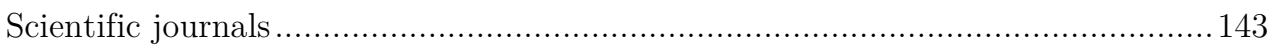

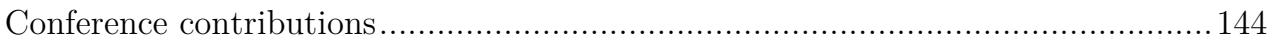

ACKNOWLEDGEMENTS ..................................................... 145

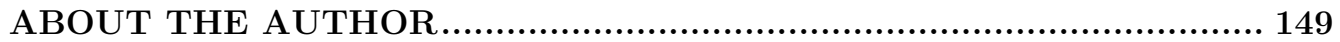





\section{1 \\ Introduction}

This thesis deals with the design, fabrication and implementation of a microfluidic chip capable of manipulating small water droplets in oil flow. The droplets are manipulated by electric forces that arise when applying an electric potential over electrodes embedded in the microchannel substrate. Different electrode geometries allow for different actuation scenarios. While dragged by oil flow, droplets can be guided along a rail, trapped at a specific location, split in two, merged to form larger droplets, and sorted at high speed based on droplet content. To put the technical developments described in this thesis in perspective, we first present a review of droplet manipulations in two-phase microfluidics in a broader sense, i.e. using other principles and designs. Manipulating droplets electrically turns out to be - not only very versatile, but also - applicable even beyond the usual AC frequencies. To understand this, we revisit the theory of electrostatics.

This introduction starts with our motivation for designing a microfluidic chip capable of electrical actuation, followed by the limitations imposed by our goals that need to be taken into account for the design. Next, a discussion of the theory of electronic actuation (electrowetting) is described shortly. Finally, it provides a more detailed guide through the thesis. 


\subsection{Motivation}

The goal of this thesis was to develop a technological platform for diagnostics of individual biological cells, which express specific proteins. A good example are cancer cells. The idea is that analyzing a single cancer cell can speed up the diagnostic process, because there would no longer be need for a cell culturing step. In advanced stages of cancer - when time is of the essence - a few cancer cells could be harvested from several milliliters of the patient's blood, the so-called circulating tumor cells, or CTCs. The speedy analysis of antigens, which are present on the cell membrane or are excreted/secreted by the cell, allows for a better targeted drug therapy.

As an example of a diagnostics tool, one can think of surface plasmon resonance imaging (SPRi). SPRi is a technique that is very sensitive to refractive index changes close to a (typically gold) substrate (Fig. 1-1). Therefore it is capable of measuring the interaction of cell excreted proteins or cell membrane molecules with analyte-specific antibodies conjugated on the gold coated prism used in SPR. The idea is to let the single cancer cell, or its excrements, interact with one of the ligand (antibody) patches and measure if there is a specific interaction and how strong the interaction is. The cell is then moved towards the next ligand patch which measures the expression of another protein, and so on. By comparing the responses on the different patches, a 'fingerprint' of the disease can be obtained.

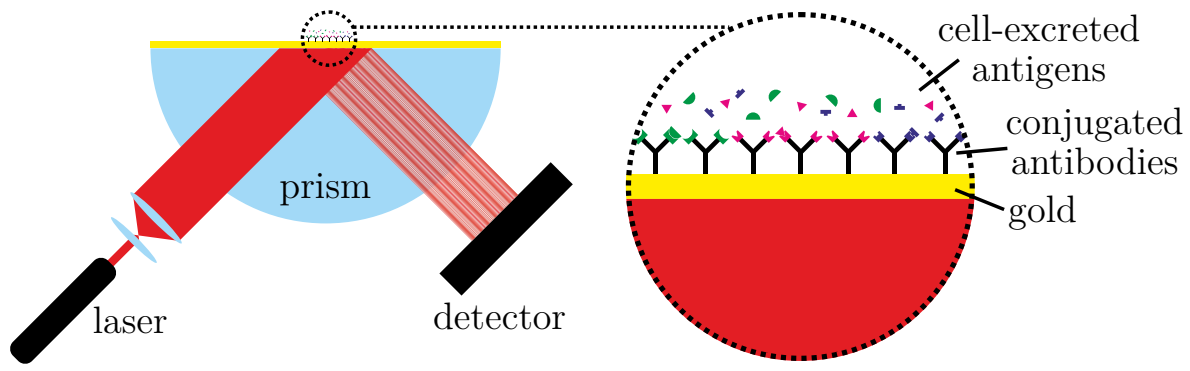

Fig. 1-1 Example of an SPR imaging scheme. Depending on the angle of incidence of the laser light and the refractive index of the volume near the gold interface a certain amount of the photonic energy will be transferred to create a surface plasmon wave. This loss of light intensity of the reflected light is then imaged on the detector. The detection principle lies in the change in refractive index that occurs when excreted molecules bind to the local antibodies on the specific spot.

\subsection{Design Criteria and Choices}

The proposed method for cell diagnostics puts some stringent limitations on the design of our device. To be able to measure the expression of a single cell it has to be completely isolated from any other cell inside the probed volume, i.e. a container. Also, to reach a sufficient concentration of analyte in a reasonable amount of time the volume of this container needs to be small, let's say in the order of 100 picoliters. This design criterion immediately brings us into the realm of microfluidics. Specifically two phase flow 
microfluidics, where water droplets are generated in an immiscible ambient fluid, e.g. air or oil, comes to mind as the easiest and most reliable technique to make these droplets.

Another problem that has to be overcome is the cell capturing efficiency. With most techniques that generate individual cells inside drops not each generated droplet will contain a cell, let alone the cell that is suitable for diagnostics (e.g. blood samples contain only several CTCs amidst millions of other cells). This brings the need for an active sorting mechanism where cell-containing droplets are separated from empty ones. In chapter 2 the existing techniques to manipulate droplets in two phase flow microfluidics are reviewed. Taking into account the force, reliability and controllability, electrostatic potential wells were decided to be the best option to reach our goals. The possibility of high speed sorting of droplets is explored in chapter 5 .

For analyzing the expression of cells one needs to screen for multiple proteins. This requires multiple small patches of printed ligands on the substrate, and the droplet needs to be transported towards these patches, held in place to interact with the patch, and be released and transported to the next patch. It would also improve the performance of our device if multiple cells could be screened in parallel. Parallel guiding and trapping is explored in chapter 3. The added interaction with a hydrophilic patch is performed in chapter 4 .

\subsection{Manipulation of droplets using electrowetting}

Manipulating small drops is not a simple task. Chapter 2 describes several techniques, each with its pros and cons, that might make it perfect for one application but unsuitable for another. Our application demands that we work with small, isolated aqueous droplets. Water droplets are naturally conductive, and even more so when physiological salt solutions are used. This contrast between conductive liquid and non-conductive ambient medium is perfectly exploited by actuation with electric fields.

\subsubsection{Electrowetting Principle}

As the name accurately suggests, electrowetting is the increase of the wettability between a conductive liquid and an (often insulated) electrode substrate caused by the electric field, applied between the liquid and the electrode. This increase of wettability is experimentally observable as a decrease of the contact angle of the droplet with respect to the substrate.

In the classical electrowetting setup a droplet is placed atop a flat electrode substrate, as depicted in Fig. 1-2a. The droplet is grounded by an immersed, conductive wire. Since the water is highly conductive, the droplet itself can be considered a grounded electrode. Then, an electric potential difference is applied between the two electrodes.

In the early days of electrowetting the water and the electrode were in contact. In this case by applying a potential an electric field is built up in the electric double layer (EDL) at the liquid-substrate interface. However, only a very small voltage $<1.23 V$ can be applied to this system before electrolysis occurs. This obstacle was overcome by covering the electrode substrate with a typically $100 \mathrm{~nm}$ to $10 \mu \mathrm{m}$ dielectric insulating layer. ${ }^{1}$ By insulating the electrode the applied potential mainly falls across the insulator, not the 
EDL. As a rule of thumb, dielectric materials can withstand $\sim 50 \mathrm{~V} / \mu \mathrm{m}$ before dielectric breakdown occurs. This means that a much higher potential may be applied before electrochemical decomposition occurs. It also ensures that a much larger amount of energy can be stored in the electric field, which is now mainly present in the dielectric material.

So, why is all this important? The power supply, i.e. battery, is able to provide energy to the electrowetting system by building up an electric field between the two electrodes. From electrostatics we know that in this process half of the energy provided by the power supply is stored in the electric field and still available for work, while the other half is lost to heating. The dissipation of electric energy effectively decreases the total free energy, which - as thermodynamics teaches us - is desirable. The electric energy $W_{e}$ that can be stored in an electric field, and is thus available for electric manipulation of the droplet, is given as $W_{e}=\frac{1}{2} C U^{2}$, with $C$ the system capacitance and $U$ the applied voltage. Altogether, we can state that a system that can store more energy in an electric field is the most energetically favorable. Therefore, the larger the capacitance the stronger the electrical manipulation.

(a)

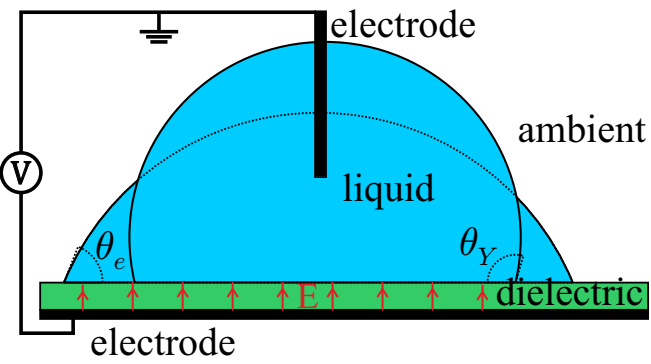

(b)

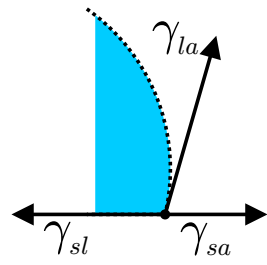

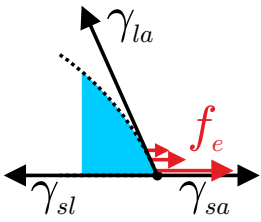

Fig. 1-2 The principle of electrowetting. (a) On the application of an electric potential, a liquid atop an insulator covered electrode decreases its contact angle to increase its ability to store electric field energy. (b) On the left, the force balance dictated by the liquid-ambient, solid-liquid and solid ambient interfacial tensions results in the droplet obtaining the so called Young's contact angle $\theta_{Y}$. On the right, the added electric tension pulling on the entire contact line spreads the droplet over the substrate. Volume conservation then dictates that the contact angle must decrease to the so called apparent electrowetting contact angle $\theta_{e}$.

\subsubsection{The electrowetting equation}

The electrowetting equation is used to describe the spreading of a droplet by application of an electric field. In the case of classical electrowetting the liquid electrode is capable of increasing the system's capacity, i.e. electric energy, by increasing its surface area in contact with the insulator. It also implies that the surface energy increases, because the total area of the droplet increases. Since the volume of the droplet is conserved during spreading this implies that the contact angle must adapt.

In electrowetting the reduction of electric energy by spreading is partly compensated by the decrease of surface energy. Energy minimization (See Appendix 1A) leads to the electrowetting equation, a.k.a. the Young-Lippman-Dupré equation, relating the contact 
angle due to electrowetting with the initial contact angle:

$$
\cos \theta_{e}=\cos \theta_{Y}+\frac{1}{2} c U^{2} / \gamma_{l a}
$$

The last part of this equation is the non-dimensional electrowetting number

$$
\eta=\frac{1}{2} c U^{2} / \gamma_{l a}
$$

which is the ratio of the electric stress and the liquid-ambient surface tension, both acting on contact line. It gives a measure of electrowetting 'strength' and enables comparison of different electrowetting experiments.

The electrowetting equation can be very useful in determining electric properties by analyzing the droplet shape. For instance, by measuring the contact angle $\theta$ as a function of voltage $U$, the thickness of the dielectric layer can be determined, assuming $\epsilon_{d}$ and $\gamma_{l a}$ are known. Or, it can be used to determine the electric force acting on the contact line simply by observing the contact angle and applying a force balance. We should note however, that the change in contact angle is merely an observable consequence of applying a voltage difference over the dielectric, which also adds an electric stress to a contact line. It is not the change in contact angle that induces a change in droplet shape or position. Since contact angles are difficult to measure in microfluidic devices, in most chapters in this thesis we will determine the electric energy directly.

Since the contact angle is determined by the balance of the solid-liquid, solid-ambient and liquid-ambient surface tensions at the contact line (see Fig. 1-2b), we could say that electrowetting modifies the liquid-solid interfacial tension. The electrowetting equation indeed seems to suggest that the electric stresses are acting directly on the contact line. This is not entirely correct, of course, because we cannot really change the nanoscopic molecular interactions between the liquid and substrate. The electric stresses are actually acting on an area around the contact line to a height comparable to the thickness of the insulating layer. Thus, zooming in on the contact angle, we should see that at the interface the contact angle is still the Young's angle, while the macroscopic angle is the electrowetting contact angle.

\subsubsection{Applications of electrowetting}

Electrowetting as described in the previous section has several useful practical applications. For instance, as described by Eral, et al. (2013)2 , AC electrowetting decreases contact line pinning. This principle can be used to enhance the shedding rate of droplets from airplane wings, preventing icicle formation, or the shedding of condensed droplets from heat collectors, ensuring an increased heat transfer rate. ${ }^{3} \mathrm{AC}$ electrowetting also enhances flow circulation inside the droplet by shaking the contact line, thus preventing suspended particles from sedimenting during droplet evaporation. As Kudina, et al. $(2016)^{4}$ show, this suppresses the coffee stain effect and concentrates the analyte after evaporation, resulting in a strong signal-to-noise enhancement for MALDI mass spectroscopy. As an added bonus, the increase of liquid-air surface area caused by the 
spreading of the droplet enhances the evaporation rate, decreasing the waiting time. Recently, electrowetting has also been used to make droplets jump from a substrate. ${ }^{5,6}$ Besides the 'grounded-wire-in-drop' electrowetting setup, several other electrode geometries have been designed for droplet manipulation. The most used geometry for realworld applications is the parallel plate geometry used in Digital microfluidics (DMF). This geometry consists of a substrate accommodating multiple, individually addressable electrodes and a grounded, parallel top plate electrode (See Fig. 1-3a). Activating an electrode when a droplet is nearby allows the creation of an electric field between the drop and that electrode. Electric energy will keep constantly increasing as the droplet covers more and more area above the activated electrode. Effectively, an electric force pulls on the contact line of the droplet until it covers the entire electrode. Using DMF allows for very accurate, on-demand control for droplet creation, transport, merging and splitting (See Chapter 2). In Movie 1-1 an example of DMF is shown using electrodes printed with silver ink using a regular inkjet printer. This 'weekend science experiment' was performed at the Physics of Complex Fluids group in collaboration with DigiBio."

Another option is the co-planar electrode geometry. This geometry puts the grounded and active electrodes in the same substrate. (See Fig. 1-3b) Besides a simpler device fabrication, another advantage is that this leaves one side open for other applications, e.g. microscopic imaging, fluorescence detection, ellipsometry or SPR imaging. The electric energy landscape is very different from the parallel plate geometry and is dependent on the droplet position relative to the gap between the electrodes. An electric energy well is formed, where electric energy is minimum when the drop is centered across the gap

(a)

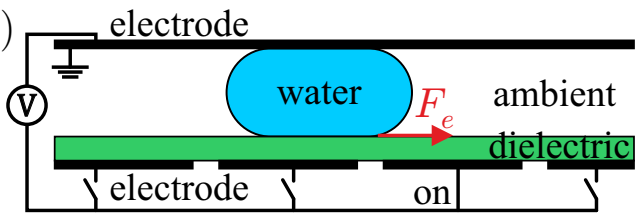

(b)

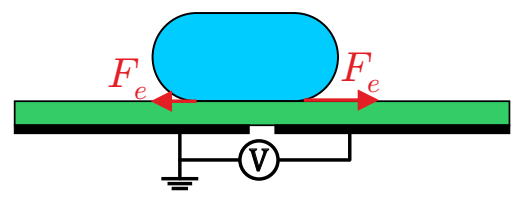

(c)

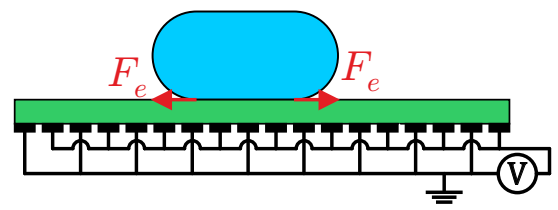

Fig. 1-3 Different electrode geometries used in electrowetting. (a) Parallel plate electrode geometry used in a Digital Microfluidics (DMF) setup. (b) Co-planar electrode geometry as used in to created Electrostatic Potential Wells. (c) Interdigitated electrode geometry.

* www.digi.bio 
between two electrodes. Effectively, the electric force will always push a droplet towards the gap. In Chapter 3 co-planar electrodes are combined with microfluidic flow for the guiding, trapping, release, sorting and splitting of droplets.

Yet another option is the interdigitated electrodes geometry (Fig. 1-3c). Here, alternatingly active and grounded electrodes with a pitch much smaller than the droplet size are incorporated in one substrate. Effectively, this geometry is very similar to the classical electrowetting geometry without the need for a grounding wire in the droplet.

\subsection{Guide through the thesis}

Chapter 2 contains a review analysis of the available techniques to manipulate droplets in two phase flow microfluidics. Passive and active techniques are discussed. Active techniques range from pressure driven to electrical, magnetic, acoustic and optical actuation. The working mechanisms and possibilities of these techniques are explained. The focus lies specifically on the manipulations required for our device: to generate, sort, guide, trap and release the droplets. Furthermore, the different techniques are compared for factors like reliability, force, controllability and cost. According to us the most predictable, controllable and reliable technique capable of performing all the required tasks in a microchannel is the use of electric potential wells.

In chapter 3 the theory and application of electric potential wells is discussed. They are used for the guiding and trapping of droplets in oil flow. Guiding is facilitated by an electrode geometry consisting of multiple electrodes that create different paths for droplets depending on which electrodes are actuated. In this case the electrodes branch into 6 paths which laterally guide droplets to 6 lanes in the microchannel. Consecutively, the guided droplets are trapped against the oil flow at a 6 by 6 array of trapping electrodes. Other actuations shown in this chapter are the splitting and sorting of droplets.

Chapter 4 is an extension of the capability to trap and release droplets. When using SPR as a diagnostics tool, the contents of a droplet will need to interact with antibodies printed on the substrate. These patches of antibodies will form hydrophilic areas in an otherwise hydrophobic channel. A dual-sided microchip is made with actuation electrodes in the bottom substrate, and hydrophilic gold patches on the hydrophobic top substrate. In this thesis, this dual-sized chip was the most challenging chip to make and therefore attention is given to the fabrication process. We focus on the trapping and release of droplets in the presence of a hydrophilic patch. Furthermore, it deals with the need to break up the oil film between the drop and the patch. Also, the relatively large pinning force generated by the hydrophilic patch of equal size as the droplet poses a unique challenge to overcome.

In chapter 5 we optimize the system for sorting droplets as described in chapter 3 to enable the sorting of droplets at high speeds. The working principle of our sorter is based on a contrast in conductivity between the drop and the continuous ambient phase, which ensures successful operation even for drops of highly conductive biological media like phosphate buffered saline. Moreover, since the electric field does not penetrate the drop, its content is protected from electrical currents and Joule heating. A simple capacitive model allows quantitative prediction of the electrostatic forces exerted on drops. The maximum achievable sorting rate is determined by a competition between electrostatic 
and hydrodynamic forces. Sorting speeds up to 1200 per second are demonstrated for conductive drops of $160 \mathrm{pL}$ in low viscosity oil. Finally, preliminary results of experiments dealing with the sorting of droplets containing fluorescent particles and fluorescently labeled cells are reported.

Chapter 6 is a more theoretical chapter on electrowetting and the difference in actuating at low or high voltage frequencies, the regime where a drop can be considered conductive or a dielectric. Motivation for this chapter is the surprising observation that our microfluidic sorter is capable of sorting non-conductive droplets, while in regular electrowetting setups the electric forces strongly decrease when going to this so-called 'dielectric regime'. By going back to the basics of electrostatics the parameters that dictate the magnitude of electric energy and electric force are described.

In chapter 7 overall conclusions are drawn over the work performed in this thesis. Also an outlook is given on the feasibility of using electric potential wells in more practical applications. 


\section{Appendix 1A: Derivation of the electrowetting equation}

The electrowetting equation is used to describe the spreading of a droplet by application of an electric field. In the case of classical electrowetting the liquid electrode is capable of increasing the system's ability to store electric energy by increasing its surface area in contact with the insulator. Since the volume of the droplet is conserved this implies that the contact angle must adapt. It also implies that the surface energy increases, because the total area of the droplet increases.

The decrease in electric energy $W_{e}$ for creating an electric field in the dielectric layer is given as:

$$
W_{e}=-\frac{1}{2} c A_{s l} U^{2}
$$

with $c=\epsilon_{0} \epsilon_{d} / d$ the capacitance per unit area, $A_{s l}$ the area where the liquid is in contact with the solid insulator, $\epsilon_{0} \epsilon_{d}$ the permittivity of the insulator, $d$ the insulator thickness and $U$ the applied potential.

The total surface energy $W_{s}$ is the sum of all interfacial areas times their respective interfacial tension:

$$
W_{s}=\gamma_{l a} A_{l a}+\gamma_{s a} A_{s a}+\gamma_{s l} A_{s l}
$$

Taking a set volume enclosing the electrowetting setup the bottom area is $A_{\text {bottom }}=$ $A_{s a}+A_{s l}$. The surface energy can now be written as:

$$
W_{s}=\gamma_{l a} A_{l a}+\left(\gamma_{s l}-\gamma_{s a}\right) A_{s l}+\gamma_{s a} A_{b o t t o m}
$$

where $\gamma_{s a} A_{\text {bottom }}$ is constant. When spreading the droplet the solid liquid interface increases with $\delta A_{s l}$. The liquid ambient area changes with $\delta A_{l a}=\cos \theta_{e} \delta A_{s l}$. The change in electric energy while spreading is:

$$
\delta W_{e}=-\frac{1}{2} c U^{2} \delta A_{s l}
$$

The change in surface energy while spreading is:

$$
\delta W_{s}=\gamma_{l a} \cos \theta_{e} \delta A_{s l}+\left(\gamma_{s l}-\gamma_{s a}\right) \delta A_{s l}=\gamma_{l a}\left(\cos \theta_{e}+\frac{\left(\gamma_{s l}-\gamma_{s a}\right)}{\gamma_{l a}}\right) \delta A_{s l}
$$

Which, given Young's equation $\frac{\left(\gamma_{s l}-\gamma_{s a}\right)}{\gamma_{l a}}=-\cos \theta_{Y}$, becomes:

$$
\delta W_{s}=\gamma_{l a}\left(\cos \theta_{e}-\cos \theta_{Y}\right) \delta A_{s l}
$$

The equilibrium contact angle is reached when $\delta W_{s}+\delta W_{e} \equiv 0$, thus when:

$$
\gamma_{l a}\left(\cos \theta_{e}-\cos \theta_{Y}\right)=\frac{1}{2} c U^{2}
$$




\section{References}

1. Berge, B., Electrocapillarity and Wetting of Insulator Films by Water. Comptes Rendus De L Academie Des Sciences Serie Ii, 1993. 317(2): p. 157-63.

2. Eral, H.B., D.J.C.M. 't Mannetje, and J.M. Oh, Contact angle hysteresis: a review of fundamentals and applications. Colloid and Polymer Science, 2013. 291(2): p. 247-60.

3. Baratian, D. and F. Mugele, Unpublished. 2017.

4. Kudina, O., B. Eral, and F. Mugele, e-MALDI: An Electrowetting-Enhanced Drop Drying Method for MALDI Mass Spectrometry. Analytical Chemistry, 2016. 88(9): p. 4669-75.

5. Cavalli, A., D.J. Preston, E. Tio, et al., Electrically induced drop detachment and ejection. Physics of Fluids, 2016. 28(2).

6. Wang, Z.T., D. van den Ende, A. Pit, et al., Jumping drops on hydrophobic surfaces, controlling energy transfer by timed electric actuation. Soft Matter, 2017. 13(28): p. 4856-63. 


\section{2}

\section{Review: Droplet manipulations in two phase flow microfluidics}

Even though droplet microfluidics have developed since the early 1980s, the number of applications that have resulted in commercial products is still relatively small. This is partly due to an ongoing maturation and integration of existing methods, but possibly also because of the emergence of new techniques, whose potential have not been fully realized. This review summarizes the currently existing techniques for manipulating droplets in two-phase flow microfluidics. Specifically, very recent developments like the use of acoustic waves, magnetic fields, surface energy wells, and electrostatic traps and rails are discussed. The physical principles are explained, and (potential) advantages and drawbacks of different methods in the sense of versatility, flexibility, tunability and durability are discussed, where possible, per technique and per droplet operation: generation, transport, sorting, coalescence and splitting.

This chapter has been published as:

A.M. Pit, M.H.G. Duits and F. Mugele, "Droplet Manipulations in Two Phase Flow Microfluidics", Micromachines 6(11), 1768-1793 (2015) 


\subsection{Introduction}

Droplet microfluidics has been around since the early 1980s, but the number of examples that have made it towards commercial applications are limited. In his 2006 overview, Whitesides ${ }^{1}$ stated that microfluidics was still in its infancy, but might offer revolutionary new capabilities for the future. Indeed great advances have been made since then, leading to commercial devices for genome sequencing, Polymerase Chain Reaction (PCR) and flow cytometry. Each of these techniques uses some form of microfluidics and droplet control for the automation of complex laboratory protocols.

The first tool required for droplet microfluidics is, of course, a system to generate droplets in the order of picoliter or even femtoliter ${ }^{2}$ volumes. To generate a droplet one needs a flow geometry (sometimes supplemented with an external field) that causes the continuous (mostly aqueous) phase to form a neck, followed by a break-up. This should preferably work in a highly controlled manner leading to a large continuous production of monodisperse droplets. Secondly, one or more methods of droplet manipulation are required, which force a droplet to execute the desired protocol. These methods do not necessarily have to be active. Some elegant passive methods have been developed for splitting, ${ }^{3,4}$ guiding, ${ }^{5}$ and trapping ${ }^{5-7}$ drops at a certain location. Thirdly, most protocols require a certain type of analysis or detection, which mostly is optical (scattering, transmission, adsorption, fluorescence, Raman, surface plasmon resonance) or electrical (measuring current, voltage, impedance).

Over the years, several excellent reviews on droplet microfluidics have been published. There are short overviews on available techniques for droplet generation, ${ }^{8-10}$ methods for single cell encapsulation, ${ }^{11}$ passive microfluidic techniques, ${ }^{12}$ manipulations using electrowetting, ${ }^{13,14}$ as well as on the fundamental challenges involved, ${ }^{15}$ on the (need for) integration of biology, chemistry and physics in biological assays, ${ }^{16-18}$ on the choice of optimal surfactants, ${ }^{19}$ and even comprehensive review articles ${ }^{20-22}$ and books ${ }^{23}$ covering the entire field of droplet based microfluidics. Also the theoretical fluid physics aspects behind microfluidics have been covered. ${ }^{24}$

The present review is oriented towards techniques that have recently become available to manipulate the droplets in microchannels - both actively and passively. We try to give an impartial view of what are the advantages of each technique, and the downsides, as far as researchers are willing to divulge these. Some of these techniques are still in the academic research stage, waiting to be used for practical and commercial applications. This paper is further organized as follows: we first discuss the existing techniques from the physics point of view, and then separately how they have been translated to passive and active manipulations for executing laboratory protocols for biophysical and biochemical purposes.

\subsection{Passive manipulation techniques}

\subsubsection{Drag force and viscous dissipation}

We define passive control as the use of a dedicated microfluidic geometry to control droplets entirely via the flow field, without any controller interference. In steady state 
flow the velocity of a droplet is governed by the drag exerted on the droplet by the continuous carrier medium. The terminal droplet velocity depends on how the two liquids flow through a channel. When a droplet is much smaller than the channel cross section the resulting terminal (i.e. steady state) velocity of the droplet equals the average local velocity of the continuous phase. In general, a small drop will follow the streamlines of the flow of the continuous phase. In Poiseuille flow this means that droplets move faster in the center of the channel.

When droplets take up a larger portion of the channel cross section they are confined by two or four walls, resulting in the formation of lubrication films around the slug. As Baroud, et al. $(2010)^{10}$ explain, the pressure drop $\Delta P$ across (and thus the terminal velocity of) slugs of droplets in a rectangular channel is influenced by corner flow of the continuous phase around the droplet. From this, it follows that not only viscosity and geometry play a role, but also the interfacial tension (thus deformability) influences the terminal velocity of the droplet. Using a microfluidic comparator, the influence of drop size, viscosity, interfacial tension and flow rate on the hydrodynamic resistance in a rectangular channel has been measured. ${ }^{25}$

As an example, let's assume a confined droplet in a microchannel that only undergoes small deformations. This assumption is often valid in microchannels where the capillary number is very small and capillary forces (surface tension) dominate over viscous forces (viscosity and velocity). Such a droplet experiences a friction force due to the confinement, balanced by a drag force due to the surrounding flow field. The drag force exerted on such a droplet can be written as $F_{d r a g}=\alpha L \mu \Delta v$, with $\alpha$ a dimensionless prefactor determined by the geometry, $L$ the characteristic length scale of the problem dependent on droplet radius and channel geometry, $\mu$ the dynamic viscosity, and $\Delta v$ the difference in velocity of the continuous and dispersed phase. For an example of such a situation the reader could examine the supplementary information of de Ruiter, et al. $(2014)^{26}$, where the drag force on a droplet confined in a hele-shaw channel geometry in oil flow is described analytically.

When droplet deformations are no longer negligible the relation between drag force and velocity is no longer linear because the deformation (and thus prefactor $\alpha$ ) becomes velocity dependent. This brings us to another related factor that can influence droplet velocity: the presence of surfactants at the interface. The circulating flow fields inside and outside the slug can transport surfactants across the interface resulting in accumulation of surfactants at the rear of the droplet. This surfactant concentration gradient at the interface results in Marangoni stresses, leading to increased dissipation, slowing down the droplet. The interplay of these different mechanisms can make it difficult to predict the terminal velocity of a slug. For instance, in the presence of surfactants it can be possible for an air bubble to have a lower terminal velocity than a water droplet. ${ }^{3}$

These basic insights can be helpful in designing passive control systems. As an example, one can use the different properties of droplets, and the resulting difference in drag forces exhibited on them, to change their behavior. For instance, drops of different viscosity will obtain a different terminal velocity. Partially confined, high viscosity droplets undergo a stronger viscous dissipation and therefore move slower than low viscosity droplets of equal radius (and confinement). This allows low viscosity droplets to catch up with high 
viscosity droplets, resulting in coalescence. ${ }^{27}$ Similarly, droplets of different radius behave differently. In the regime of terminal velocity, a drop with smaller radius will move faster than a larger drop. This is because in Poiseuille flow the velocity of the continuous phase is higher near the center of the channel. Therefore a smaller droplet in the center of the channel will obtain a larger average velocity than a larger droplet. The same principle allows for the hydrodynamic separation of particles of different sizes. ${ }^{28}$ In most cases however, droplets will neither have a different viscosity, nor a different size.

\subsubsection{Geometric structures}

It is possible to design the geometry of a channel such that the flow field alters the drop behavior. For instance the splitting of a droplet can be facilitated by dividing one channel into two branches, or using a pillar as a flow obstruction in the channel. Depending on channel widths and hydraulic resistances, this allows to create pairs of identical daughter droplets, or to split the volume of the mother droplet into a larger and a smaller fraction. ${ }^{4}$ As shown by the Vanapalli group, ${ }^{29}$ so-called microfluidic parking networks can be used to let one droplet alter the flow field (and hence also the fate) for consecutive drops. When given the choice to enter different channels, a droplet will tend to follow the path of least hydraulic resistance. However, if that path contains a constriction much smaller than the droplet size, trapping of the droplet will occur, because the pressure drop over the parallel channel is smaller than the large Laplace pressure required to pass through the constriction. The droplet then effectively blocks that channel for consecutive droplets, forcing them to take the parallel channel. Depending on ratios of channel resistances a regime can be found where for instance every first drop is captured, or even regimes where the first droplet goes into the bypass channel, effectively increasing the resistance of this parallel channel, so that only the second or third droplet are captured. By letting consecutive drops coalesce, Bithi, et al. $(2014)^{7}$ also showed the ability to control the droplet volume accurately (Fig. 2-5b), or change the (bio)molecular concentrations inside the droplet. Other geometric structures, like an array of traps, can be built in a wide microchannel as well; in that case chance dictates the trapping of droplets (Fig. 2-5a). ${ }^{6}$

Another great example of passive channel geometries has been shown by Korczyk, et al. $(2013)^{30}$ Only by changing the flows their channel geometries are capable to precisely meter a volume from a larger drop, merge droplets to create droplets of different concentration, delay a droplet and have a droplet shift register.

\subsubsection{Surface energy wells}

After a droplet is generated in a microchannel it is often confined by two walls and takes a pancake or disc shape. Alternatively, if it is confined by four walls it becomes a slug. If there had been no walls (or other external forces) the droplet would have taken the shape of lowest surface energy, a sphere. Therefore it stands to reason that if the squeezed droplet was able to decrease its interfacial area that such change would be energetically favored. Creating a small hole in the top of a microchannel allows a drop to reduce its curvature, and thus its interfacial energy by locally expanding into the hole. Once a droplet is lodged in this surface energy well it will require force to pull it out (See Fig. 2-1a). This makes it possible to trap a drop against the drag force, ${ }^{31}$ or split a droplet. ${ }^{32}$ 
Building further on this idea, also thin trenches or 'rails' were created, effectively creating elongated (directional) energy wells. These allow to steer a droplet in flow along these rails, ${ }^{33}$ or to fuse two droplets by using two converging rails. ${ }^{34}$ Other researchers used similar rails for the fusion and sorting of droplets. ${ }^{35}$ As is the case with other passive techniques: it is difficult to release the drop once trapped, or to select which way to steer a drop. Using active laser forcing (See section 2.3.5) these problems can be overcome. ${ }^{5}$

\subsection{Active manipulation techniques}

Active manipulation entails changing the behavior or fate of a droplet (in flow) by an external, user-controlled mechanism. Below we discuss the more common techniques that are used for active control, selected on their proven ability to control droplets accurately and reliably. Our main focus will be on techniques that can control a droplet after it has been formed, but some of these techniques can also be used before or during droplet generation.

\subsubsection{Pneumatic membrane (Quake) valves}

A common material for fabrication of microfluidic devices is polydimethylsiloxane (PDMS), a polymer that can be molded using standard soft lithography techniques. ${ }^{36,37}$ Multiple layers of PDMS are used as the basis for pneumatic membrane valves. One layer contains the microchannel structure through which the liquids will flow. Another layer consists of gas filled channels which cross over the microchannel. At the point where the channels cross, the two layers are separated by just a thin membrane of PDMS. By increasing the pressure of the gaseous phase the membrane expands into the liquid channel (See Fig. 2-1b). When enough pressure is applied the liquid channel can be blocked, effectively forming a pneumatic valve. ${ }^{38}$ This technique has been applied for creating a peristaltic pump,${ }^{38}$ for the sorting of droplets, ${ }^{39}$ and for droplet generation. ${ }^{40}$ Especially for droplet generation this technique has some advantages over other methods. Single droplets are generated on-demand, and the amplitude and duration of the pressure pulse can be used to control the size of the droplet. ${ }^{41}$ Placing multiple droplet generators in series allows merging droplets of different content and size. In turn this enables the creation of large sets of droplets, covering wide ranges in concentrations and content. ${ }^{42}$ Using many valves, such droplets can be individually guided and stored in a multiplex array of 95 wells for cell culturing or PCR analysis. ${ }^{43}$

\subsubsection{Electrical techniques}

\subsubsection{Dielectrophoresis}

Dielectropheresis (DEP) is the motion of polarizable objects caused by application of a non-uniform electric field. In microfluidics, DEP is mainly used to sort small particles with a different dielectric constant $\epsilon_{r}$ than the surrounding medium. ${ }^{44}$ There are multiple ways to understand how DEP works. For small droplets the easiest way to visualize DEP is to look at the charge distribution within the droplet. As we know, opposite charges attract. Thus, in an electric field any polarizable medium will orient its electric charges such that they oppose the electric field. Three main ways for a medium to polarize are: 
ionic, dipolar and electronic. If the medium contains ion pairs, like a salt dissolved in water, these can quickly shift the charges at the droplet interface. If the molecule is in itself a dipole, like a water molecule, it can rotate and orient itself against the electric field. Much faster, within each atom the positively charged nucleus and negative charged electron cloud can shift slightly to oppose the electric field.

Regardless of the polarization mechanism, the end result is a droplet that can be considered as a dipole which is oriented against the electric field. In an inhomogeneous field this means that the electrostatic attraction is larger at the side of the dipole where the electric field is stronger, resulting in a net force in the direction of increasing field strength (See Fig. 2-1c). There are analytical expressions for the DEP force on small spherical objects. ${ }^{45}$ For different (droplet) shapes and volumes numerical modeling is needed to calculate the electric field and potential, and subsequently the DEP force.

In practical DEP applications an electric field inside a microfluidic channel is generally created by applying a voltage across two (or more) electrodes just outside the channel. A simple way to get gradients in the electric field is to use a pointed electrode where all the electric field lines converge. Especially interesting for two-phase flow microfluidics is that water droplets in an electric field can on average be considered as a large dipole. The consequence hereof is that not only small particles, but also water droplets in oil can be sorted with DEP, even at high speeds $(>1 \mathrm{kHz}) .{ }^{46}$

When dealing with larger volumes instead of dielectric droplets or particles, it can also be insightful to describe DEP (and electrowetting as well, see Section 2.3.2.2) via a capacitive energy approach. From electrostatics we know that when a voltage source charges a capacitor, the total electrostatic energy of the system decreases: $W_{e}=-\frac{1}{2} C U^{2}$ with $C$ the total lumped capacitance and $U$ the applied potential. In microfluidic systems, a pair of electrodes next to a microchannel with either water, oil or another dielectric material in between also forms a capacitor. Since the electrostatic energy (locally) decreases with increasing capacitance, it is thermodynamically favorable to have a system with large capacitance. Using a lumped capacitance model, i.e. taking into account the capacitances of all different media between the electrodes, $W_{e}$ can be calculated. The simplest case is a parallel plate capacitor, for which $C=\frac{\epsilon_{0} \epsilon_{r}}{d} A$, with $d$ the thickness of the dielectric between the electrodes and $A$ the electrode area. Since displacement of the medium between the electrodes (e.g. oil) by one that has a higher polarizability (e.g. water) increases the lumped capacitance, it is energetically favorable for highly polarizable media to move towards the region of high electric field strength.

\subsubsection{EWOD / DMF}

In the context of microfluidics, ElectroWetting-On-Dielectric (EWOD) (also known as digital microfluidics, DMF) generally uses arrays of electrodes insulated by a thin dielectric layer to control conductive droplets. ${ }^{47}$ This enables highly accurate, on-demand control over individual droplets like transportation, generation, splitting, coalescence and mixing. ${ }^{22}$

The term electrowetting originates from the observation that a grounded water droplet atop an insulated electrode spreads when a voltage is applied, effectively decreasing the droplet contact angle with the substrate and thus 'wetting the surface better'. This 
concept is explained by the Young-Lippmann model, which balances the surface energy cost with the gain in electrostatic energy when a droplet spreads. The model allows to calculate the (oil/water) interfacial tension, or the insulator thickness when measuring the contact angle as a function of voltage. ${ }^{48}$ As an applied theoretical example the model also predicts the position of the droplet in an electrode wedge structure, dependent on applied voltage and the resulting droplet contact angle. ${ }^{49}$ In droplet based microfluidics, however, it is difficult or even impossible to determine the contact angle. This makes application of the Young-Lippmann model less useful. To explain the droplet motion, it might be better to use an electrostatic model, in this case the same lumped capacitive model as mentioned before for DEP.

Although it is not always mentioned, EWOD is very similar to DEP. ${ }^{50}$ Both techniques use the difference in response of the media to an applied electric field. One difference is that EWOD mainly uses aqueous droplets which are conductive due to the addition of ions. The high conductivity allows to consider the droplet as an electrode with resistance but negligible capacitance. This also makes a second difference between DEP and EWOD clear: the insulating layers are required to prevent the short circuiting of the electrodes. The continuous phase can be air, or an oil of low permittivity. In presence of a potential difference across electrodes but absence of a water droplet, the capacitance is intrinsically low. However, if the oil or air is replaced by a conductive drop, the total applied voltage now falls across the thin dielectric, insulating layers. The capacitance of the droplet can be ignored, and the electrostatic energy of the system can be calculated using the a similar formula as before: $W_{e}=-\frac{1}{2} \frac{\epsilon_{0} \epsilon_{r}}{d} A U^{2}$, but now with $d$ being the total thickness of the insulating layers, not the distance between electrodes. By switching on an electrode from the array of electrodes, the droplet will find a minimum energy by spreading across the activated electrode, increasing its capacitive area. The resulting force pulls on the threephase contact line allowing to move droplets (See Fig. 2-1d).

To further show the similarity between DEP and EWOD, the conductive liquid in EWOD can be described with an effective permittivity $\epsilon_{e f f}=\epsilon_{r}-j \sigma / \omega \approx \sqrt{\epsilon_{r}{ }^{2}+\sigma^{2} / \omega^{2}}$, with $\sigma$ the conductivity and $\omega$ the applied voltage frequency. At low conductivity or high frequency $\epsilon_{\text {eff }}$ reduces to $\epsilon_{r}$. This means that the water droplet can no longer be considered as a perfect conductor, and thus that the capacitance of the aqueous phase can no longer be neglected. Since the dielectric constant of the water phase must be taken into account EWOD has effectively become DEP. Unfortunately, for EWOD setups this generally means that the electric force decreases tremendously. In chapter 6 we will show ways to use electrowetting in this 'dielectric regime'.

The main advantage of EWOD is the sheer number of droplet manipulations that are possible on-demand. The fact that it works well with conductive droplets makes it ideal for biological and cell applications. Another advantage of EWOD is that it is a relatively basic electromechanical technique, which ensures that the control over water droplets is accurate, switchable, high speed and predictable. EWOD can be integrated in more complex (control) systems by using state-of-the-art techniques from the immense field of electronics. Nice examples are for instance the ability to print electrodes on thin films, allowing the manipulation of droplets on bent surfaces ${ }^{51}$ Also real-time detection of the droplet position by measuring the capacitance of a set of electrodes,${ }^{52}$ or electronic paper 
displays $^{53}$ illustrate this point. Also, thin film transistor arrays used in liquid crystal displays have been used for EWOD, giving control to over 4000 electrodes with impedance spectroscopy for droplet position detection. ${ }^{54}$

There are also some disadvantages to consider when using EWOD. One is the high voltage that is required for thick dielectric layers. Another is that relatively complex electronic systems are required when using many individually activated electrodes. Also the longevity of a device is strongly dependent on the dielectric materials used. Many different materials have been used aiming for good hydrophobicity, low hysteresis and high resistance to dielectric breakdown. Some researchers have focused on making the dielectric as thin as possible, to allow use of low voltages. This would make EWOD compatible with standard low voltage electrical systems (no need for expensive amplifiers and switches), while still allowing for high speed droplet actuation. ${ }^{55}$ Others have used relatively porous dielectrics like PDMS which are infused with the continuous oil phase, ensuring low hysteresis and sufficient hydrophobicity. ${ }^{26,56}$ It is interesting to see how an open source project like DropBot brings researchers together to make EWOD cheaper, automated, more reliable and effectively crossing the divide between laboratory research and commercial application. ${ }^{57}$

\subsubsection{Electrostatic potential wells}

EWOD devices are mostly used as large arrays of electrodes which completely control every motion of each droplet. In an attempt to acquire the strengths of both worlds, the high throughput of two-phase flow channel-based microfluidics has been combined with the individual drop control achieved using electrical actuation. ${ }^{26}$ Two phase flow allows for high speed droplet generation and the transport of droplets over large distances without the need for many electrodes. By having dielectric covered electrodes below the microchannel, the accurate on-demand control of EWOD is used to manipulate (conductive) aqueous droplets in flow. A disadvantage, as compared to EWOD alone, is that the ability to transport droplets upstream is lost.

The electrode geometries in this technique are chosen to be co-planar electrodes separated by a gap that is comparable to the thickness of the dielectric layer. When a potential is applied across such a gap, a passing droplet will try to equalize the areas of droplet contact above the electrodes since this maximizes the lumped capacitance. Effectively the electrodes form an energy well, where the electrostatic energy is minimal if the droplet centers over the gap (See Fig. 2-1e). The similarity with surface energy wells (Section 2.2.3) is clear, and therefore this method was coined 'electrostatic potential wells'. The technique has been able to trap, coalesce and release droplets in flow, split a droplet in two equal volumes, guide droplets to different lateral positions on electrostatic rails, ${ }^{26}$ and sort droplets at $>1 k H z .{ }^{58}$

\subsubsection{Pre-charging}

A third electrostatic manipulation technique that needs to be mentioned is the precharging of droplets. Several ways for precharging droplets have been described in literature. One method applies a DC electric field perpendicular to the flow in the channel. The positive and negative charges (ions) in the droplet separate to either side of the droplet. While the charges are separated the water droplet is split in two at the crossing 
of a T-junction, resulting in two oppositely charged water droplets. ${ }^{59} \mathrm{~A}$ second method uses two closely spaced electrodes below the channel. A positive or negative DC voltage is applied across the electrodes as a droplet passes and covers both electrodes. An opposite charge $q$ is induced in the droplet at the active, insulated electrode interface. The charges originate from the grounded electrode which has no insulating layer (See Fig. 2-1f). The charges remain in the droplet as it detaches from the electrodes resulting in a charged droplet. ${ }^{60}$

Charging a droplet in itself has little use. A secondary electrode pair further downstream the channel is required to apply another electric field. In this second electric field charged droplets will feel an electric force $\overrightarrow{\boldsymbol{F}}_{e}=q \overrightarrow{\boldsymbol{E}}$, with $\overrightarrow{\boldsymbol{E}}$ the electric field strength. Negatively charged droplets move towards the positively charged electrodes and vice versa. This technique is capable of sorting of pre-charged droplets into three different outlet channels at 3 drops per second. ${ }^{61} \mathrm{~A}$ secondary utilization of this technique arises from the coalescence of droplets. Two oppositely charged droplets will feel an attractive electrostatic force enabling an easier coalescence of droplets, even when the droplets are covered by surfactants. ${ }^{59}$

\subsubsection{Magnetic manipulations}

Similar to DEP where polarizable materials are influenced by an electric field, so can magnetic particles be manipulated by a magnetic field. One example is the magnetic tweezer (See Fig. 2-1g). Here, an electromagnet above the channel in combination with a ferromagnet built in the substrate below the channel generate a magnetic field with increasing intensity towards the ferromagnet. Paramagnetic particles inside droplets will orient to oppose the magnetic field and move towards the region of high magnetic field strength. In some cases the particles are capable to drag a droplet with them, which allows the movement of a droplet. In other cases the particles are extracted from the droplet and later merged with a consecutive droplet. By functionalizing the particles, for instance with antibodies, this allows the execution of biological assays. ${ }^{62,63}$

By integrating stripes of paramagnetic PDMS below a microfluidic channel, magnetic field gradients can be created around the stripes by providing an external, homogeneous magnetic field. Water droplets loaded with paramagnetic microparticles will then move towards the stripes, where the magnetic field gradient is highest. This technique provides an energy landscape very similar to the surface energy rails and the electrostatic potential rails discussed in Section 2.2.3 and 2.3.2.3, and has been used to guide and sort droplets in oil flow along the rails and trap and merge droplets at the end of the magnetic rail. ${ }^{64}$ Similarly, rotating external magnetic fields have been used to let ferrofluidic droplets perform automatic tasks, effectively creating logic gates (See section 2.4.6). ${ }^{65}$

\subsubsection{Acoustic waves}

\subsubsection{Surface acoustic waves}

As the name suggests, a surface acoustic wave (SAW) is an acoustic pressure wave that propagates across a substrate. It is created by interdigitated electrodes on a piezo-electric substrate. The resonance frequency of the interdigitated transducer (IDT) depends on the 
(a) SURFACE ENERGY WELL
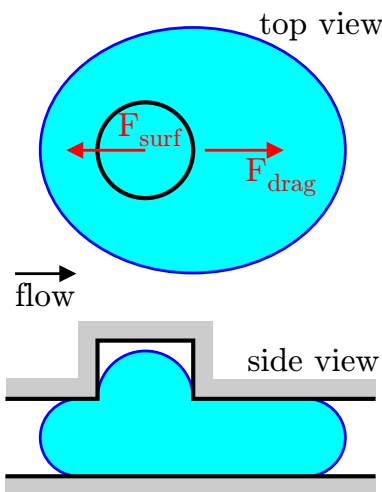

(d) EWOD / DMF

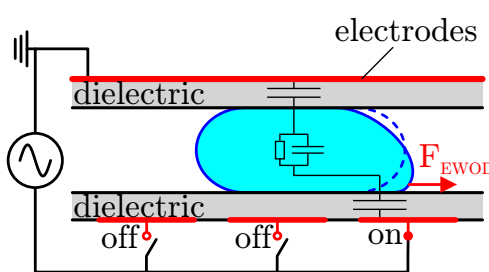

(g) MAGNETIC TWEEZER

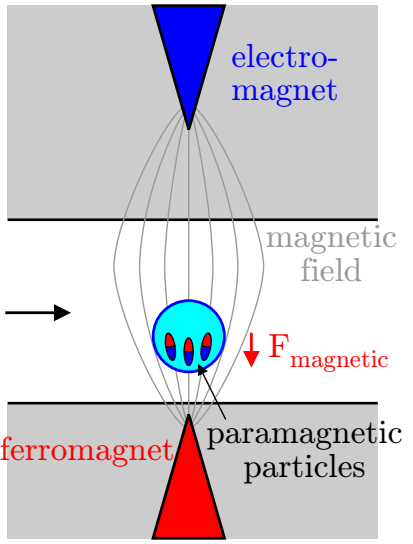

(b)PNEUMATIC MEMBRANE VALVES valve open

ow pressure $\mathrm{P}_{0}$

valve closed

$$
\mathrm{P}_{0}+\Delta \mathrm{P}
$$

(e)

ELECTROSTATIC POTENTIAL WELL

side view

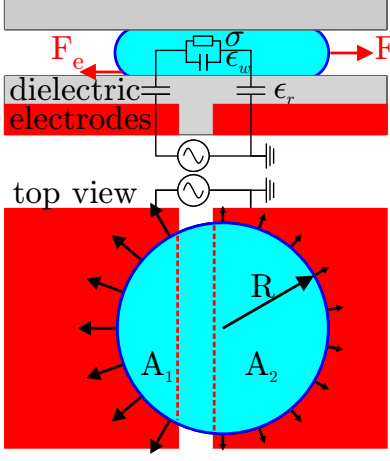

(h) SURFACE ACOUSTIC WAVE

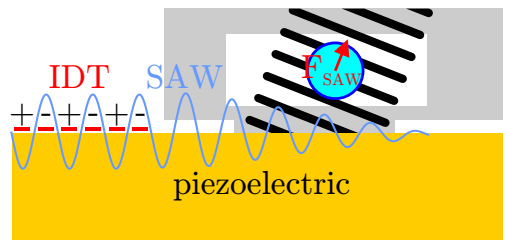

(i) BULK ACOUSTIC WAVE

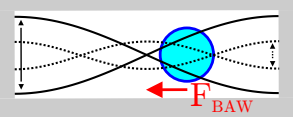

piezoelectric (c)

DIELECTROPHORESIS

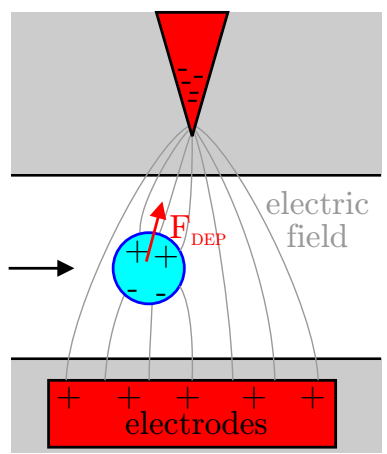

(f)

PRE-CHARGING

side view

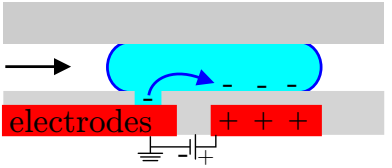

forcing charged droplet top view

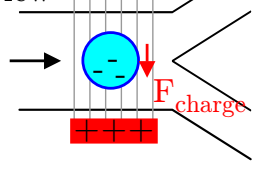

(j) OPTICAL TWEEZER

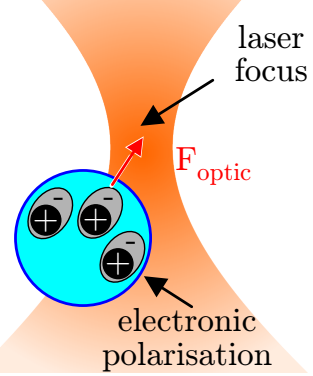


Fig. 2-1 on the left shows a schematic overview of the droplet manipulation techniques. (a) a squeezed droplet can decrease its interfacial energy by expanding in a hole; (b) increasing the air pressure above the local water pressure expands the membrane into the crossing microchannel, effectively blocking it; (c) a polarizable medium will move towards the region with highest electric field intensity; (d) the electrostatic energy will be minimal when a droplet covers the activated electrode; (e) a droplet passing two electrodes finds a minimum energy when centered above the electrodes; $(\boldsymbol{f})$ by taking charges from the open electrode a droplet can be precharged. A precharged droplet will move towards the oppositely charged electrode; (g) paramagnetic particles move towards the highest magnetic field strength and can drag a droplet along with them; (h) the interdigital transducer creates a surface acoustic wave that is attenuated at the PDMS pillar, creating an upwards pressure wave that can move droplets; (i) at specific frequencies the piezo can create resonating pressure waves in a channel, forcing a droplet towards the antinodes; (j) a medium with better polarizability at optical frequencies than the surrounding medium will move towards the region of high intensity laser light.

pitch between the electrodes and the characteristic velocity at which a sound wave travels across a specific surface material. When a potential is applied over the IDT at the resonance frequency a high pressure Rayleigh wave of $\mathrm{MHz}$ frequency and nanometer amplitudes is created, which propagates across the substrate. An encounter with a fluidic channel attenuates the wave by coupling its energy into the fluid. This results in a (standing or travelling) bulk pressure wave propagating through the liquid at an angle (the Rayleigh angle) that is determined by the differences in acoustic impedance (material density and wave speed) of the media. This bulk wave can generate an acoustic radiation force on droplets or cause acoustic streaming within a liquid. The first manipulates droplets, the latter influences particles in flow.

While the technique for creating SAWs has been demonstrated in the $1960 \mathrm{~s},{ }^{66}$ it has only recently been rediscovered as a means for external control in microfluidics. Firstly for the focusing of beads ${ }^{67}$ and soon after for the sorting of droplets. ${ }^{68}$ Very recently the technique has been used for steering of slugs ${ }^{69}$ By using a tapered electrode geometry localized pressure fields can be created in the channel. In this local field a droplet can be trapped temporarily and then merged with a consecutive droplet. ${ }^{70,71}$ Another way to localize the region where the pressure wave will occur is by using a PDMS pillar to connect the IDT substrate with the channel (See Fig. 2-1h). Only at the pillar the SAW will be attenuated and form an upwards pressure wave. This clever design allowed the sorting of droplets containing cells from empty droplets at a rate of $3 \mathrm{kHz} .{ }^{72}$ Combining multiple tapered IDTs with multiple pillars enables the sorting towards multiple outlet channels. Besides forcing individual droplets, the SAWs are also capable of providing enough pressure to break up a thin co-flowing stream of water in oil. This enabled to generate cell-containing droplets directly from co-flow. ${ }^{72}$

Numerical simulations can be used to predict the acoustic field in a channel, ${ }^{72}$ and the displacement of a surface by the SAW can be measured using Laser Doppler vibrometry. ${ }^{70}$ 
Recently, another optical technique has been developed which is able to measure the acoustic field in a microchannel directly. ${ }^{73}$ Advantages of SAW are that only small voltages are required to create very large forces, and that pressure waves will manipulate any object in the channel, independent of permittivity, conductivity, refractive index or size. A disadvantage could be that the brute force from SAWs is not localized and SAWs are therefore not capable for highly accurate control. Within these limits it is still possible to perform several protocols that do require accuracy, like splitting ${ }^{71}$ a droplet in two, by using multiple IDTs and carefully tuned voltage actuation sequences.

\subsubsection{Ultrasonic Acoustophoresis}

Besides SAWs it is of course also possible to create bulk acoustic waves by simply using a flat piezo-electric surface in contact with the channel walls, instead of the interdigitated transducer. This directly creates bulk acoustic waves (BAWs) in the microchannel. Unlike SAWs, BAWs are restricted to discrete, harmonic resonance modes such that the wavelength fits the channel dimension (See Fig. 2-1i). If a particle or droplet is large enough it will move towards the nearest anti-node. This allows to sort large objects from small particles. BAWs have also been used for stretching and mixing of droplets, ${ }^{74}$ as well as for sorting and coalescing droplets. ${ }^{75}$

\subsubsection{Optical manipulation techniques}

The use of focused laser spots generates various possibilities for droplet manipulation. Light waves can interact with objects in different ways. A focused light beam is in fact nothing more than an electric field gradient with the highest field strength in the center. Very similar to how dielectric objects respond to an electric field, as described in Section 2.3.2.1, so do objects of high permittivity move towards the center of the highest electric field strength created by the laser. The difference is that electric field frequency for laser light lies around 3 to $7 \cdot 10^{14} \mathrm{~Hz}$. The orientational and ionic polarization mechanisms of molecules cannot follow these frequencies and the permittivity thus only depends on the electronic screening abilities of the atoms. Perhaps the best known example of this principle are optical tweezers (See Fig. 2-1j). ${ }^{76}$ A collimated laser beam enters an infinity-corrected objective lens and is focused into a narrow beam in the focal plane. This focal spot is used to trap and transport a high permittivity particle or droplet. By changing the angle at which the collimated beam enters the objective the focal spot is translated laterally, while changing the incoming beam to slightly diverging or converging translates the focal spot axially. The technique is not limited to just water of course, but can for instance also manipulate liquid crystals, ${ }^{77}$ as long as there is a gradient in refractive index. To be able to manipulate multiple droplets or particles, multiple individually changeable beam wave fronts are required. Towards this end spatial light modulators have been used to create up to 400 dynamically addressable optical tweezers. ${ }^{78}$ A disadvantage of optical manipulation techniques is that the absolute force that can be generate is quite low in the order of $100 \mathrm{pN}$ or lower. ${ }^{79}$ This means optical techniques are probably only useful at low flow rates.

Electrostatic interaction is not the only influence light has on particles. Light waves also refract at the interface of a spherical particle of different refractive index than the 
surrounding medium. And because photons are known to have momentum this means that photons which are scattered or absorbed actually transfer this momentum to the particle or droplet and force it in the direction of laser beam propagation, as well as towards the beam center. This principle makes it possible to selectively sort droplets towards different outlets. ${ }^{80}$ To prevent optical trapping of the particle, the laser beam is not focused.

A different method to use focused laser light to manipulate droplets is using the light to locally heat the interface of surfactant covered droplets. This heating increases the local interfacial tension of the droplet, probably by removing or destroying the surfactant, effectively creating an interfacial tension gradient at the droplet interface. As a result Marangoni flows are created around the laser hot spot yielding a force that pushes the droplet away from the hot spot. This technique has been used for influencing droplet

generation, coalescence and fusion. ${ }^{81}$ It also has been used in combination with the passive surface energy wells described in section 2.2.3 to transport droplets from one rail to another, and to dislodge trapped droplets from their surface energy trap. ${ }^{5}$

The final optical technique worth mentioning is used for on-demand generation of droplets by using intense pulsed laser light to create rapidly expanding cavitation vapor bubbles. ${ }^{82}$ The cavity comes to existence when the strong optical field induces breakdown of the water molecules, forming a plasma. The energy quickly dissipates and the heat generated in this process creates a quickly expanding cavity vapor bubble of which the pressure wave moves through the water and perturbs a nearby oil-water interface. The water pushes into the oil phase to form an aqueous droplet. Droplet generation rates of up to $10 \mathrm{kHz}$ and droplet volumes of $1-150 \mathrm{pL}$ were realized. The authors report volume deviations of just $1 \%$, which would make this technique one of the most accurate ways for droplet generation.

\subsection{Required manipulations}

\subsubsection{Droplet generation}

The creation of droplets is the basis of any two-phase flow microfluidic chip. ${ }^{8-10}$ Especially in the early days of droplet microfluidics many different channel designs have been thought of to generate droplets. Most common techniques are based on hydrodynamic interaction between the two immiscible phases, where the continuous phase applies shear on the eventually dispersed phase.

One option is the $\mathbf{T}$-junction, ${ }^{83}$ where two perpendicular channels bring the two phases together. When the front of the dispersed phase fluid intrudes into the main channel, a pressure drop occurs between the front and rear of the emerging droplet. The droplet grows under the balance of pressure, interfacial tension and shearing force until the neck at the rear becomes thin enough - and the pressure low enough - to break into a small droplet. ${ }^{84}$ In practice, the T-junction creates slugs of droplets in the exiting channel with a minimal length of twice the channel width, or larger if the pressure in the dispersed phase channel is increased. Smaller droplet sizes can be obtained at higher flowrates, but these are generated by a Plateau-Rayleigh instability, which is generally not considered 

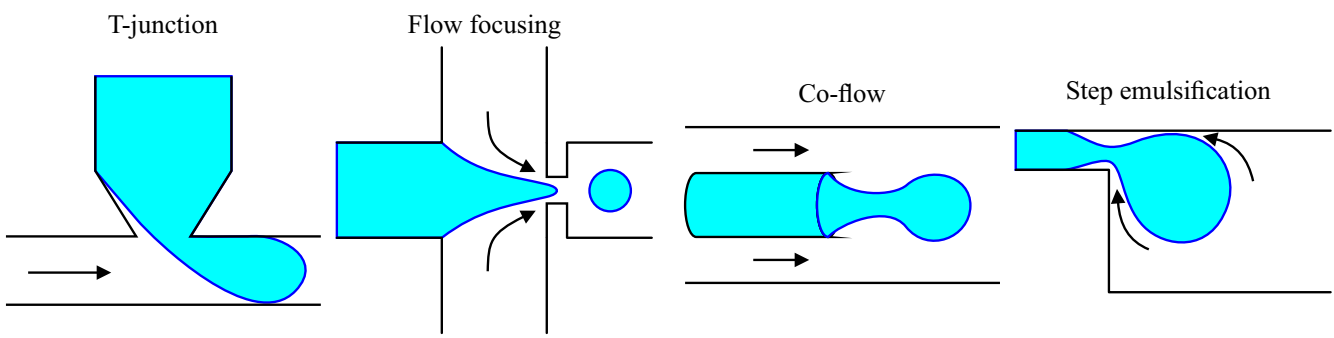

Fig. 2-2 Schematic representation of the most popular droplet generation methods. Black arrows indicate the flow direction of the continuous phase.

to be the best method to create monodisperse droplets.

Another option for generating droplets is the flow focusing device (FFD). ${ }^{85}$ In an FFD the dispersed phase is located in a center channel while the continuous phase enters from two perpendicular side channels. In this way, the dispersed phase is squeezed between the two continuous phase flows. By shear stress a neck is formed and broken from the dispersed phase creating a droplet. Often a smaller orifice through which all the liquids must flow is positioned behind the FFD. This creates a high local shear stress at the orifice, which ensures the neck is broken in this region. In practice, droplet size and generation frequency are influenced by the FFD geometry, the viscosities of the two phases and the flowrates. The droplet sizes are more uniform as compared to techniques that use the Plateau-Rayleigh instability to break the neck. Droplet generation frequencies can go up to several $\mathrm{kHz}$.

Very similar to the FFD, drops can also be generated by a co-flowing geometry. ${ }^{86}$ In this geometry a thin (glass) capillary or (metal) needle is inserted as a center channel inside the main channel. The dispersed phase - often water since it wets the glass capillary well - enters via the capillary and is segmented by the shear force of the external continuous fluid on the dispersed phase. At higher flowrates a jet is formed and droplet break up is again caused by Plateau-Rayleigh instability. ${ }^{87}$ One advantage is that the dispersed phase is completely surrounded by the continuous phase. This ensures that the dispersed phase does not interact with the channel walls, which can significantly increase the durability of the microfluidic channel when using biological media. Another advantage is the ability to create double or even triple emulsions-inside-emulsions. ${ }^{88}$

Another method is called step emulsification. ${ }^{89,90}$ This technique is based on the fact that a soon-to-be-dispersed liquid filling a thin channel is always confined by the (nonwetting) side walls. A confined liquid is forced into strong curvature, resulting in a large Laplace pressure drop across the interface. If a region is offered where the channel expands in both width and height (a step) the tip of the water phase will accelerate into the wider channel to decrease its high curvature. As it accelerates a neck is formed behind the droplet as the continuous phase flows upwards in the channel. ${ }^{91}$ When the neck has become thin enough, it eventually breaks due to Rayleigh-Plateau instability. The step can also be a gradual change in height where the gradient influences the eventual droplet size. ${ }^{34}$ The step emulsification method has the advantage that the drop size is mainly controlled by the geometry and the resulting change in Laplace pressure, and less influenced by 
pressure differences between the liquid phases. This allows to parallelize the step geometry and thus generate a high throughput of reasonably uniform droplets. ${ }^{34,89}$

Active control over the droplet generation frequency can easily be achieved by changing the flow rates or pressures of the liquid phases. A very elegant technique for actively controlling the droplet generation frequency is dielectrophoresis (DEP). ${ }^{92}$ By applying a high-frequency voltage across four electrodes positioned around the FFD an electrostatic force is applied on the water interface. Increasing the voltage increases this force, pulling the water interface further downstream in the FFD and effectively increasing the droplet generation frequency. Compared to syringe pumps, electrical control allows for much faster switching between generation frequencies.

So far, the discussed techniques generate continuous streams of drops. To generate single droplets on-demand active control is required. Firstly, the dispersed phase has to be static for this application. This can be achieved by stopping the flow, but in case of syringe pumps, control can be hindered by elastic effects from the tubing and is therefore not very fast or stable. A simpler approach is to use a pressure controller for the dispersed liquid phase. Stopping the soon-to-be-dispersed liquid phase can be facilitated by using a tapered channel: as the liquid traverses through the tapering channel, its curvature increases. Ultimately the increasing Laplace pressure will match the applied pressure on the liquid phase, causing the flow to stop. By applying a short pressure pulse a droplet can be generated on demand. Other active methods for on demand drop generation include electrowetting, ${ }^{13,}{ }^{93,94}$ SAWs, ${ }^{95}$ pneumatic valves, ${ }^{41,}{ }^{96,97}$ and laser-induced cavitation. ${ }^{82} \mathrm{~A}$ nice feature of the pneumatic valves is that consecutive pneumatically controlled droplet generators can directly combine different solutions into one droplet at controllable ratios. ${ }^{42}$
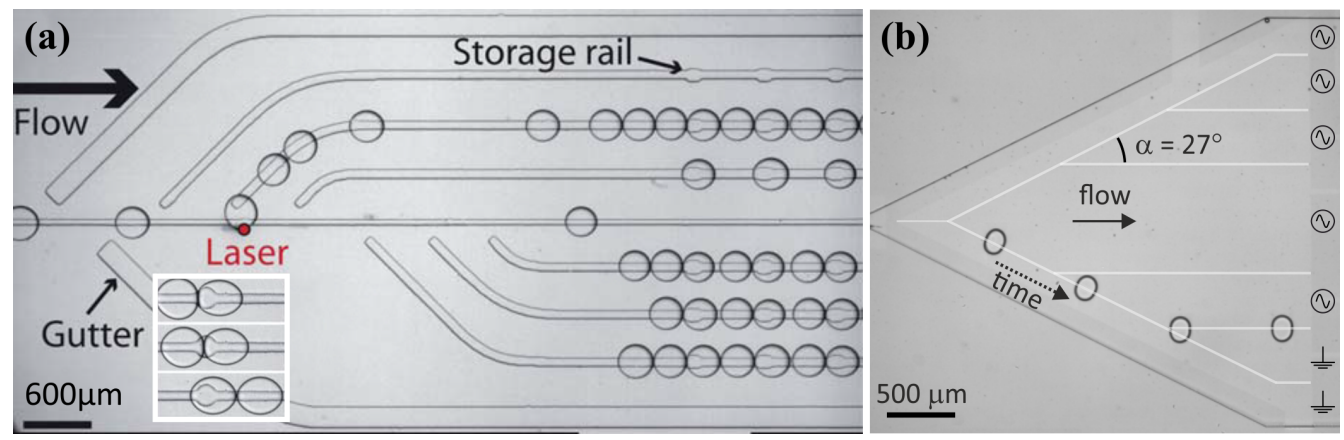

Fig. 2-3 (a) Guiding of droplets by surface energy rails. The laser spot is used to select and force droplets onto the rail. A local widening of the rail serves as a deeper surface energy well where trapping will occur. A droplet can be pushed out of the well by a consecutive droplet. Adapted from [5] with permission of The Royal Society of Chemistry. (b) Guiding of droplets by electrostatic potential rails. Depending on which electrodes are activated a droplet will be guided towards one of six lateral positions in the channel. Adapted from [26] with permission of The Royal Society of Chemistry. 


\subsubsection{Droplet transport / guiding / steering}

In some protocols it is required to transport a droplet to a specific location, for instance towards a position where it should be held for incubation, or where it can be analyzed by some optical technique, or the starting location of another protocol on the same chip. Both passive and active techniques have been devised to transport droplets in the desired direction. Arguably the most accurate technique that offers external, on-demand control over droplet transport is EWOD. Protocols for electrode actuation can be preset, but also changed by the user depending on what is needed. Optical tweezers are able to trap a droplet in the focal waist and by moving the focal spot the droplet can be transported.

The simplest passive technique for droplet transport is to let it follow the flow of the continuous phase. Microfluidic walls or obstructions determine where a droplet goes. More interesting are manipulation techniques that can be used in combination with flow. In Section 2.2.3 surface energy rails were discussed that can passively guide droplets, assisted by flow. Inspired by the simple physics behind these surface energy rails, electrostatic potential rails have been designed to actively guide droplets towards six different lateral positions in a wide channel, depending on which electrodes are activated. ${ }^{26}$ An intricate network of microchannels and pneumatic valves has been used to direct flows of drops towards 95 wells independently. ${ }^{43}$

\subsubsection{Droplet sorting}

The sorting of droplets is perhaps the most important application in microfluidics, especially for chemical or biological applications. Most researchers use fluorescence detection to determine whether a droplet should be sorted or not, called fluorescence assisted droplet sorting (FADS). Whether it is a fluorescently labeled cell in a droplet that needs to be separated from empty droplets, or proof whether a chemical reaction has taken place, fluorescence is an easy-to-use and fast technique enabling detection at high speeds.

The sorting of droplets is actually a subset of guiding droplets, so all previous techniques for active droplet transport can be used for sorting as well. Albeit a relatively slow process, the surface energy rails achieved active sorting control by laser forcing. ${ }^{5}$ Also the novel method of magnetic rails has room for improvement at sorting rates of 4 drops per minute. Pre-charging of droplets allowed to sort droplets at $3 \mathrm{~Hz}$ so far ${ }^{61}$ Standing SAWs have been shown to reach sorting frequencies of $222 \mathrm{~Hz} .{ }^{98}$ By using a pneumatic valve to close the waste outlet (with low hydrodynamic resistance) droplets can be sorted towards the other outlet at $250 \mathrm{~Hz} .{ }^{39}$ The electrostatic energy rails have been converted into a smaller electrode geometry allowing for active sorting at $1200 \mathrm{~Hz} \cdot{ }^{58}$ As mentioned before DEP was one of the first applied, active sorting techniques for droplets, ${ }^{46}$ later improved with another electrode geometry and laser detection to accomplish active sorting up to $2000 \mathrm{~Hz} \cdot{ }^{99}$ But the technique that is able to apply the largest force on a droplet is the travelling SAW, able to sort at $3000 \mathrm{~Hz}^{72}$

It must be noted that not all experiments have been done on comparable droplet systems. For instance the electrostatic energy rails used droplets with a two times larger radius than the DEP experiments, approximately multiplying the drag force that needs to be 

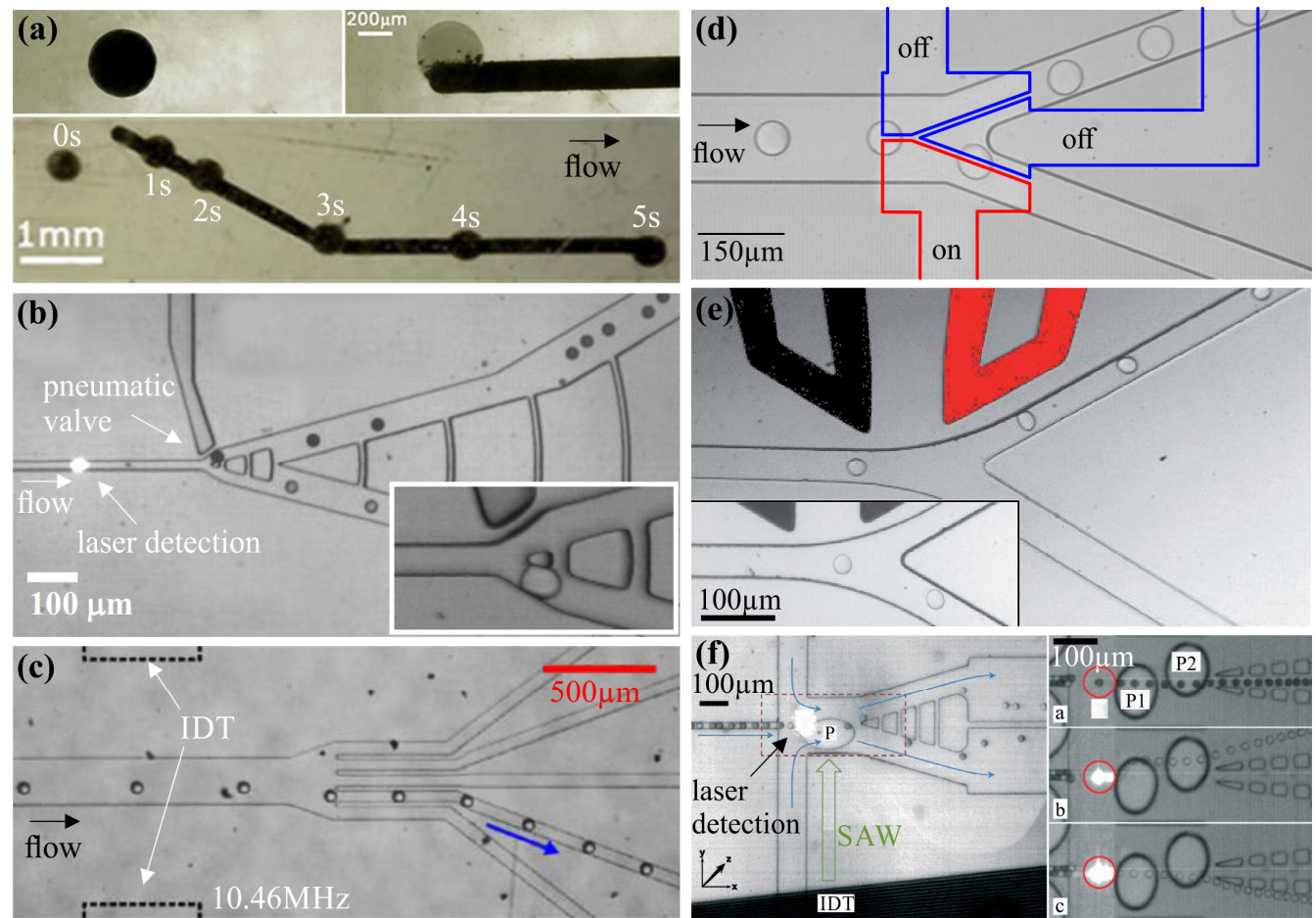

Fig. 2-4 Active sorting of droplets. (a) Magnetic particles in droplets force the droplet towards the magnetic rail when a magnetic field is applied over the entire chip. With kind permission from Springer Science+Business Media: Microfluidics and Nanofluidics, Selective handling of droplets in a microfluidic device using magnetic rails. 2015, 19, 1, 141-153, Teste, B. et al. (b) Applying a pressure to the pneumatic valve changes the path of least resistance from the upper to the lower channel. Reprinted with permission from [39]. Copyright 2014, AIP Publishing LLC. (c) Depending on the frequency of the SSAW droplets will be forced towards one of the five outlets. Adapted with permission from [98]. Copyright 2013, American Chemical Society. (d) By switching on or off the central electrode the electrostatic potential rails will force a droplet to the upper or lower channel. Adapted with permission from [58]. Copyright 2015, AIP Publishing LLC. (e) By activating the electrode (red) an electric field gradient is created forcing water droplets to the upper channel. In the inset the droplets go to the lower channel by default. Adapted from [99] with permission of The Royal Society of Chemistry. (f) left: The IDT generates a SAW, which is attenuated at the PDMS pillar. This locally creates a upwards pressure wave that forces a droplet to the upper channel; right: Using two IDTs and two pillars allows to sort to multiple outlets. Adapted from [72] with permission of The Royal Society of Chemistry. 
overcome by four. Also, for the travelling SAW experiments the authors claim that the maximum applied force was not limited by the technique itself, but by the fact that more force could harm the droplet content.

\subsubsection{Droplet trapping and release}

Holding droplets at a specific location in a microchip during flow can be used for several protocol steps. One step could be an incubation, where a droplet needs to wait for a chemical or biological process to reach completion. Or a step where droplets need to be analyzed over a longer time, which is easier if the droplets are not moving. As an example one could think of following the interaction kinetics of a (droplet based) protein to a ligand bound on one of the channel walls, using the change in refractive index near the surface as the signal (e.g. using SPR).

Optical tweezers can be used to trap one droplet at a time, and a two-electrode geometry is able to create an energy well and hold a droplet using DEP. ${ }^{100}$ Here however, only the more practical trapping techniques are mentioned, specifically the ones that are able to trap multiple droplets simultaneously which should allow for multiplex assays. For practical reasons, like re-using a chip or analyzing droplets off-chip, it is also useful to be able to release a droplet after analysis is complete.

Geometrical structures are the simplest method of trapping droplets. ${ }^{6}$ The microfluidic parking networks are a typical example of a passive, geometrical technique for trapping droplets at specific on-chip locations. ${ }^{29}$ Concentration gradients of trapped droplets can be created, and the trapped volume can also be rectified. ${ }^{7}$ Releasing of the droplets is not mentioned by the authors, but could perhaps be possible by reversing the flow.

Arrays of surface energy wells (holes in the microchip substrate) have shown their ability to trap multiple drops. ${ }^{33}$ One disadvantage is that the trapping is determined by chance and thus non-specific. This issue has been resolved by combining the traps with surface energy rails and laser forcing, allowing to select which droplets will be trapped at which position on the chip. The success of this method was demonstrated via the creation of an array of trapped droplets with increasing concentration gradient. ${ }^{5}$ The droplets can be released by increasing the flow rate, ${ }^{31}$ which can also break up the droplet, leaving behind a controlled volume in the trap, ${ }^{32}$ or, as before, the droplet can be actively released by laser forcing. ${ }^{5}$

An intricate network of microchannels and pneumatic valves has been used to route flows through the microchannels and selectively guide droplets towards 95 wells. The droplets can be loaded one by one, and as the authors mention, their chip allows for automated cross-contamination free release and recovery of the reaction products from the individual chambers for downstream analysis. Combined with the ability to easily create concentration gradients of droplet content, this technique can be very useful for automating multiplex laboratory protocols. ${ }^{43}$

It is clear that EWOD is intrinsically capable of trapping and release, since it controls all motions of the droplet. The only flow-based technique shown to be able to trap and release multiple droplets on demand without the need for geometric obstructions is the electrostatic potential well. By applying a potential over two channel-wide electrodes separated by a zig-zagging gap, droplets can be trapped at each lateral location where the 


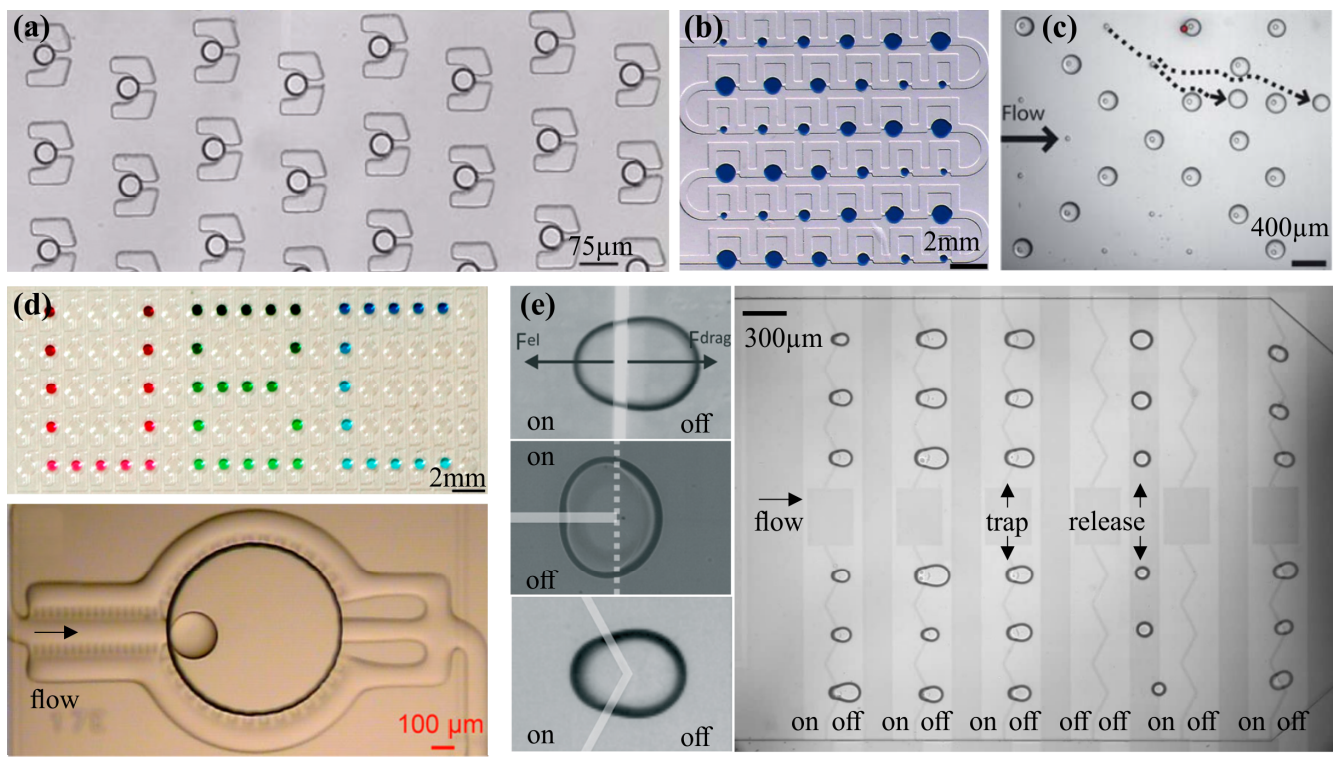

Fig. 2-5 Overview of trapping mechanisms of droplets in oil flow. (a) array of passive geometric traps. Reversing the oil flow releases the droplets. Adapted from [6] with permission of The Royal Society of Chemistry; (b) array of multiple, consecutive microfluidic parking networks of different volume. Reprinted with permission from [7]. Copyright 2014, AIP Publishing LLC; (c) surface energy wells, for trapping droplets and release by laser forcing. Adapted from [5] with permission of The Royal Society of Chemistry; (d) top: gradients of droplet content created and guided by pneumatic membrane valves to 95 individually addressable traps; bottom: magnification of the passive trapping geometry. Adapted with permission from [43]. Copyright 2012, National Academy of Sciences, USA; (e) left: three different electrode geometries for electrostatic potential wells; right: six by six electrostatic potential well arrays for the trapping and release of 36 droplets. Adapted from [26] with permission of The Royal Society of Chemistry.

gap forms a tip. Upstream the electrostatic potential rails are used to transport droplets laterally in the channel towards the desired trapping location. The angle of the zig-zag structure ensures that a droplet is corrected for errors in lateral position. One zig-zagging electrode geometry forms a column where 6 drops can be trapped on-demand by switching just one electrode. Releasing of the 6 droplets is simply done by turning off the active electrode. By placing multiple zig-zagging structures after another arrays of droplet traps are created. ${ }^{26}$

\subsubsection{Droplet splitting / fission}

The on-chip splitting of droplets could have several purposes. For instance if the volume of a droplet needs to be reduced, or if a protocol requires two identical droplets of which one for instance serves as the blank / control experiment. The first requires the asymmetric splitting of a droplet in controlled volume ratios, the second requires the 
splitting in two equally sized droplets. Since water in air or oil has a relatively high surface tension sometimes surfactants are added to the oil and/or water phase to reduce the interfacial tension which facilitates droplet splitting.

Basically, each technique used for the generation of droplets (Section 2.4.1) is capable of breaking up the droplet phase. Pneumatic valves can break up an existing droplet while it passes, or a flow focusing junction with smaller orifice or higher continuous flow rate can break a droplet into multiple smaller drops.

Many passive break up methods use a combination of flow and geometric structures. Splitting slugs of droplets can be done by dividing a channel in two, so a droplet can break up at the $\mathbf{T}$-junction. Breakup can be facilitated by adding a pointy structure to the T-junction. ${ }^{3}$ The volume ratios can be predetermined by having different hydrodynamic resistances of either outlet channel. Very similarly, a pillar obstruction in the channel can split droplets. Again changing the relative pathway resistances by placing the pillar off-center determines the volume ratio of the split droplets. ${ }^{4}$
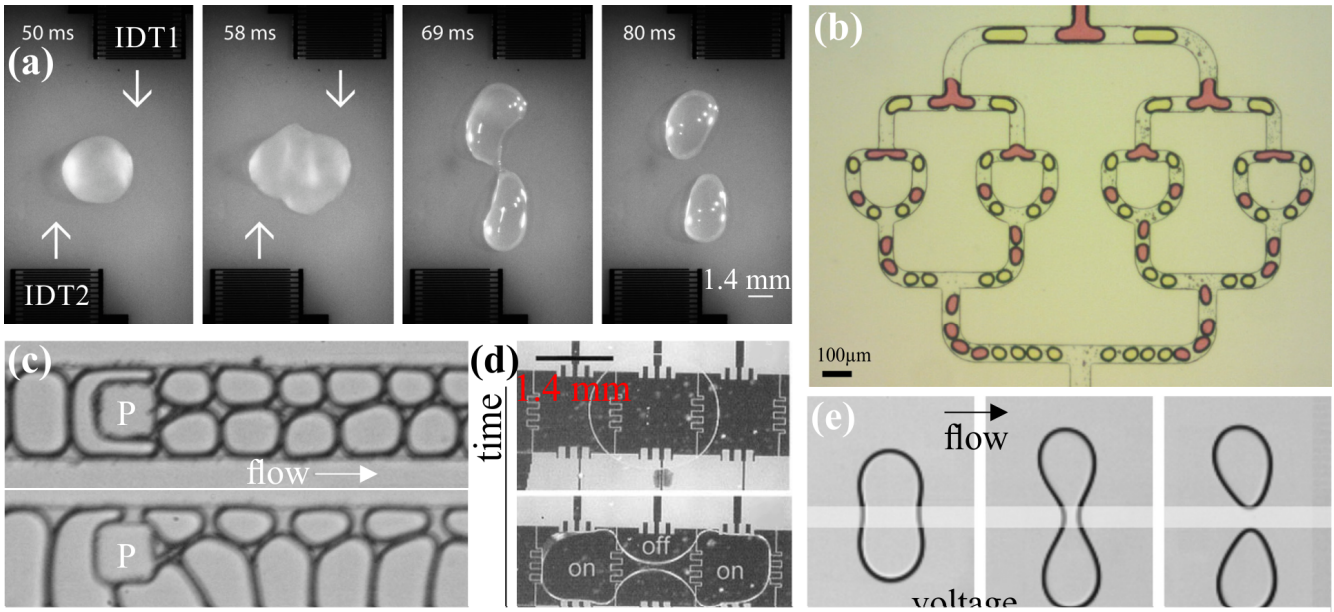

Fig. 2-6 Overview of splitting methods. (a) Two off-axis IDTs create two SAWs that apply a torque on a $3 \mu \mathrm{L}$ droplet, splitting it in two. Adapted from [71] with permission of The Royal Society of Chemistry. (b) Passively, using a multiple consecuative Tjunctions, a slug can be split into 16 equal sized droplets. Reproduced from [3] with permission of The Royal Society of Chemistry. (c) top: a pillar in the center of a channel splits droplets into equal volumes; bottom: an off-center pillar splits droplets in unequal volumes. Reprinted with permission from [4]: Link, et al., PRL 92 5, 2004. Copyright 2004 by the American Physical Society. (d) By activating a row of three EWOD electrodes a drop will elongate. After deactivating the central electrode, a bridge is formed, splitting the droplet in two equal volumes. Copyright 2003 IEEE. Reprinted, with permission, from [13]. (e) Similar to EWOD, a droplet in oil flow will split in two equal volumes if the voltage across the electrostatic potential rails is high enough. The drag force assists in the breakup of the neck. Reproduced from [26] with permission of The Royal Society of Chemistry. 
Active techniques capable of splitting droplets are quite rare. EWOD is good example for the splitting into equally sized droplets, even without need for surfactants. ${ }^{13,}{ }^{101}$ By activating 3 (or more) electrodes in a row a droplet will be elongated. Deactivation of the center electrode ensures the droplet wants to reduce its contact area with that particular electrode, while still being pulled towards the outer electrodes. This results in the droplet splitting in two equal volumes, or in discrete volume ratios depending on how many, and which electrodes used. Similarly the electrostatic potential wells have also been able to split a droplet in two equal volumes. ${ }^{26}$

SAWs can be used for droplet splitting as well. By using two off-axis IDTs on either side of a droplet SAWs can be created that apply a torque on the droplet. Low amplitude SAWs enable the stretching or rotation of a droplet. When followed by higher amplitude SAWs a droplet can be split in two. ${ }^{71}$ Depending on the ratio of amplitudes of each SAW it is possible to alter the eventual volume ratio of the split droplets. It must be noted that in this case the droplets were in the order of microliters, and not the more conventional picoliter range in droplet microfluidics.

\subsubsection{Droplet merging / coalescence / fusion}

Many protocols require the merging of droplets, e.g. two droplets with different content coalescing in order to start or stop a chemical reaction, or to dilute a sample. ${ }^{9}$ Passive techniques are available to aid in droplet coalescence. A simple passive approach is to widen the microchannel, and thus reducing the flow rate locally. This effectively reduces the distance between two consecutive droplets and promotes coalescence when the channel width is reduced once more. ${ }^{102}$ Theoretically, all that is required for droplets to coalesce is to bring them close together, and wait for the thin film between droplets to be squeezed out. Therefore any technique that can transport or trap droplets (Section 2.4.2 and 2.4.3) is capable of bringing two drops together and merge them. For instance optical forcing ${ }^{103}$ and $\mathbf{S A} \mathbf{W}^{70}$ have been used to trap a droplet until a secondary droplet flows against it. Optical tweezers have been used to fuse two femtoliter droplets together, ${ }^{104}$ while the more powerful SAW technique has also been used to push two microliter sized drops together. $^{71}$

Frequently, and especially in biological applications, surfactants are added to the oil and/or water phase, which separates droplets and helps to ensure that each droplet is one small reaction container less influenced by neighboring droplets. Surfactants can also mitigate wetting problems, and can provide a means for exchanging small molecules between droplets through the continuous phase. ${ }^{19}$ Specifically fluorinated surfactants can create highly stable emulsions and are capable of storing droplet emulsions for multiple years. ${ }^{105}$ To still be able to coalescence these surfactant stabilized droplets an external force that destabilizes the interface is required. For instance, as a chemical method an extra inlet for a destabilizing alcohol can locally increase the chance of coalescence. ${ }^{106}$ As a passive method, gaseous bubbles have been placed in between multiple water droplets of different content, serving to separate trains of droplets. Since a confined gaseous bubble moves slower through a channel than the surfactant covered water droplets, the water droplets are pushed together. Over time the thin oil film between the droplets is squeezed out and the droplets coalesce. ${ }^{3}$ Active coalescence is mostly achieved by electrical 

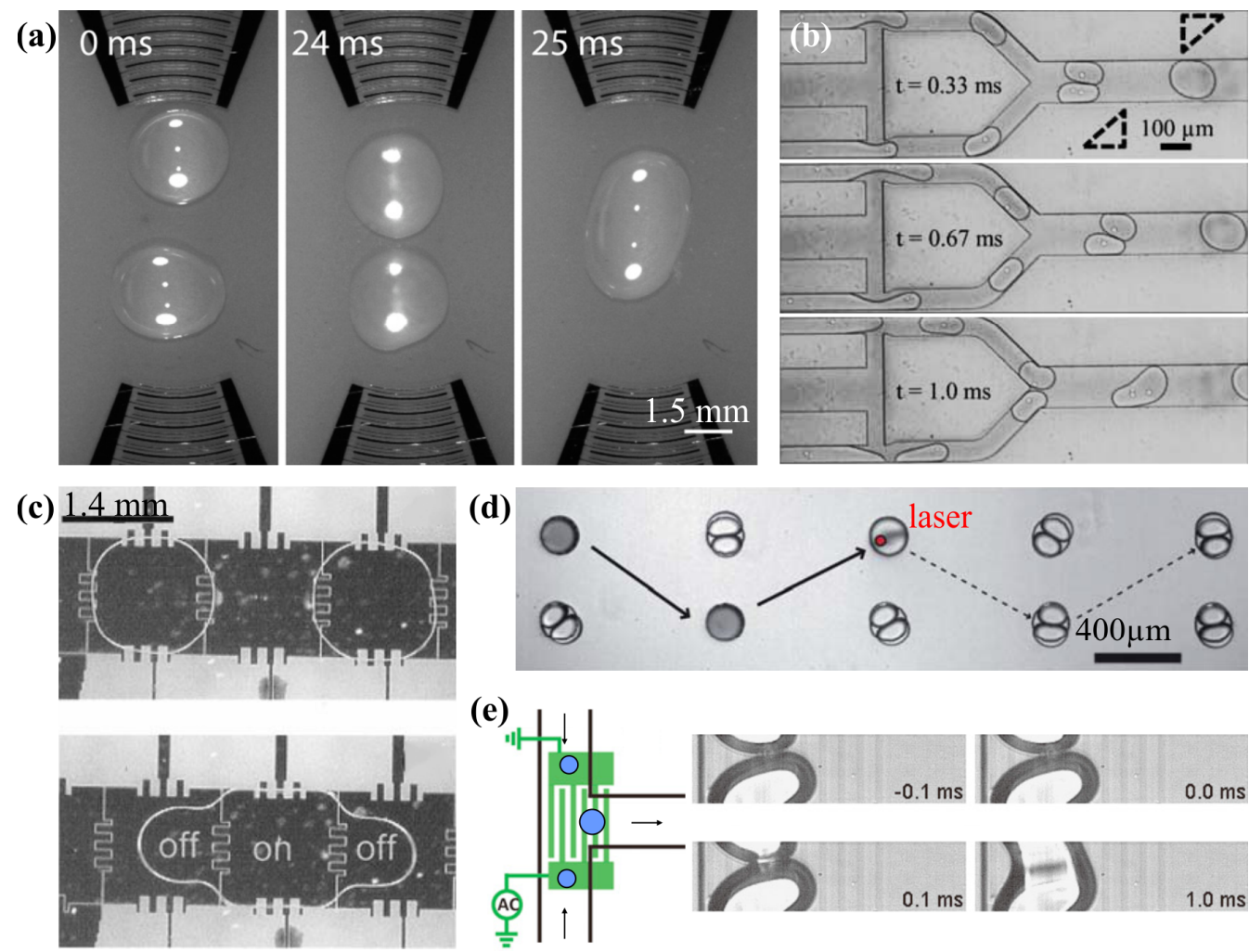

(d)

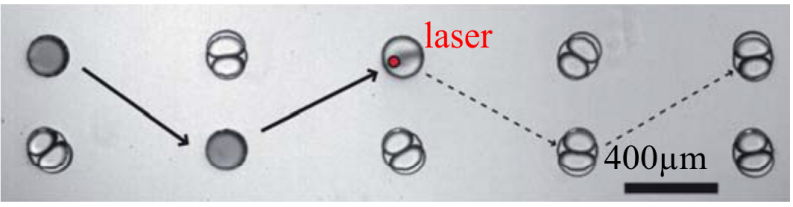

(e)

Fig. 2-7 Overview of droplet merging techniques. (a) Merging two microliter sized droplets using two SAWs focused by a tapered electrode geometry. Adapted from [71] with permission of The Royal Society of Chemistry; (b) Electrocoalescence of two cellcontaining, surfactant-covered droplets, while travelling through the electric field created by two electrodes as indicated by the dashed triangles. Coalescence frequency is $>1 \mathrm{kHz}$. Adapted with permission from [107]. Copyright 2014 by John Wiley 8 Sons, Inc; (c) EWOD electrode geometry used to bring two droplets together after splitting. Copyright 2003 IEEE. Reprinted, with permission, from [13]; (d) After trapping two surfactantcovered droplets with different content in surface energy wells, they are merged using a laser spot focused at the droplet-droplet interface. Adapted from [5] with permission of The Royal Society of Chemistry; (e) Similar to electrocoalescence, interdigitated electrodes below a microchannel will merge twosurfactant-covered droplets within milliseconds. Adapted with permission from [93]. Copyright 2011, AIP Publishing LLC.

actuation (electrocoalescence). First, two droplets are brought in close proximity for instance by the channel geometry. By using a DEP-like electrode geometry, two droplets in an electric field will polarize and charges accumulate at the surface. At the interface between the droplets the charges are opposite (if the droplet pair is aligned in the direction of the electric field), which results in an electrostatic force that brings the droplets closer 
together. The thin oil is squeezed out until the droplets merge. Applying a high frequency $(\mathrm{O}(\mathrm{kHz}))$ electric field perturbs the interface between the droplets increasing the efficacy of merging. ${ }^{107}$ In the same manner for EWOD and electrostatic potential wells two droplets have to be brought towards neighboring electrodes and the AC frequency of the applied voltage helps to destabilize the interface and speeds up the coalescence of droplets. ${ }^{13}$ Interdigitated electrode structures are capable of merging surfactant covered droplets on-demand within milliseconds. ${ }^{93}$ Surface energy wells have also been used to trap two surfactant covered, highly stabilized droplets. Laser forcing was required to break the interface and merge the droplets. ${ }^{5}$

\subsubsection{Droplet logics}

So far, in all active techniques the external control is governed by computers and electronics. Understandably so, since digital logics offer fast and reliable actuation. It could, however, make sense to build (part of) the logics into the microfluidic chip, where the presence or state of one drop is influenced by the state of other droplets, or the state of the chip. Theoretically, this could allow droplets to perform more complex routines automatically and determined by the current state of the microfluidic device. As an example, pneumatic membrane valves have been used to open and close different channels and force droplets to execute a certain protocol depending on which valves are activated or not. ${ }^{108}$ This allowed the creation of NOT, NAND and NOR gates, flip-flops, oscillators, self-driven peristaltic pumps, and a 12-bit shift register. Another method generates gas bubbles in the channel, and uses the hydrodynamic interaction between bubbles to automatically perform logic operations, forming AND/OR/NOT gates, a toggle flip-flop, a ripple counter, timing restoration and a ring oscillator. ${ }^{109}$ As a purely passive technique, a channel geometry has been designed that serves as a shift register for water droplet in oil. ${ }^{110,111}$ Very recently, ferrofluidic droplets atop a substrate incorporating multiple permalloy geometries were shown to automatically perform logic operations when a continuous rotating magnetic field is applied. ${ }^{65}$ This enabled AND, OR, XOR, NOT and NAND logic gates, fanouts, a full adder, a flip-flop and a finite-state machine without any external control. Specifically the interaction of serial and parallel logic gates could be useful in droplet microfluidics to perform the required protocols.

\subsection{Discussion / Conclusion}

One protocol step that is not extensively covered in this review is mixing. The homogenization of droplets with volumes in the picoliter regime generally requires $\sim 1 \mathrm{~s}$, assuming a molecular diffusivity of $5 \times 10^{-10} \mathrm{~m}^{2} / \mathrm{s}$, as is typical for small molecules. And that is not taking into account internal flows inside the droplet that are created by velocity gradients at the interface. It has been shown that droplets traversing a channel can passively mix within $25 \mathrm{~ms} .{ }^{112}$ Faster passive mixing (within $2 \mathrm{~ms}$ ) can be achieved by letting the droplet traverse a meandering channel: mixing is then further enhanced by the internal flow patterns. ${ }^{113,114}$ Active mixing could be achieved through application of an $\mathrm{AC}$ voltage, either in EWOD or in DEP. EWOD droplet mixers using electrode arrays have been demonstrated in closed chips ${ }^{115}$ while perturbation of the interface of a sessile 
droplet was found to enhancing mixing as well. ${ }^{116}$

The absolute forces in surface energy wells are comparatively small, which limits the operation speeds. However, the technique is easily understood, easily implemented in microfluidic devices and, perhaps it's best attribute, passive. This makes the surface energy wells very cheap to implement, since no expensive peripheral equipment (pressure regulators, amplifiers, DAq cards, laser optics etc.) are required.

EWOD (DMF) is a technique that offers the most versatile control, being able to perform all protocol steps mentioned in this review. It is also one of the more powerful techniques. The durability of EWOD still appears to be an 'Achilles heel', where dielectric breakdown or wetting can occur during prolonged application of high voltages. In general, DEP has the electrode geometry outside the channel, and therefore does not have such issues with durability. This geometry does however require (much) higher voltages, and cannot generate forces as large as with EWOD. Moreover with DEP it is not possible to perform each of the protocol steps.

SAW offers the largest forces from all techniques discussed, making it stand out as a powerful technique for sorting. Perhaps more focus could be put on making the high intensity pressure fields more localized in the channel, e.g. in a range comparable to the droplet radius, to obtain more precise control for the more subtle protocol requirements like splitting or trapping.

Pneumatic membrane valves offer an entirely different way of implementing the required protocol steps. The valves are capable of performing all the drop manipulations discussed in this review. Specifically the ability to actively change the flow direction of the continuous phase is quite unique as an active control method. One downside of the membrane valves is that they are generally an order of magnitude slower in operation than the electronically actuated methods of drop manipulation.

Optical techniques in general offer much smaller forces than the other techniques. Increasing the light intensity can generate larger forces, but also causes heating inside the liquid. Still, this heating can be used to generate droplets at high rates, or to assist in the coalescence of droplets. The use of focused laser light has also been demonstrated to be effective for several manipulations in case of surfactant covered droplets.

In short, many different methods have been devised to manipulate droplets in microchannels, each with their own specific (and sometimes inherent) advantages and drawbacks. We have given an overview of techniques that are well-established by now, and of methods which we currently consider to be amongst the most promising or ingenious. For many applications it would be desirable to use only passive techniques; each droplet would then simply execute its process without any need for outside control or feedback. Unfortunately, for the more complicated protocols that is simply impossible and external control is required. It is up to the chip designer to decide which technique will suit his purposes best, finding the optimum between the technique's possibilities, actuation speed, power, accuracy, reliability, ease-of-use, safety and costs. Table 2-1 gives an overview of the specific positive and negative aspects for each technique.

In some cases high performance applications have been realized by combining multiple techniques, as exemplified by the combination of (passive) surface energy wells and (active) laser forcing, or (active) EWOD and (passive) two-phase flow. EWOD has also 
been combined with antibody-coated paramagnetic particles (active) for the execution of complex immunoassays. ${ }^{117}$ The combination of (passive) microfluidic parking networks with (active) pneumatic membrane valves has resulted in parking networks with active control for merging, storage and release while reducing the number of required pneumatic valves. ${ }^{118}$ More of such developments towards high standard lab-on-a-chip applications can be expected, since none of the mentioned techniques seem to limit the application of another technique (a possible exception being the combination of SAW and EWOD, where it might not be practical to have EWOD electrodes on a piezo-electric material). 


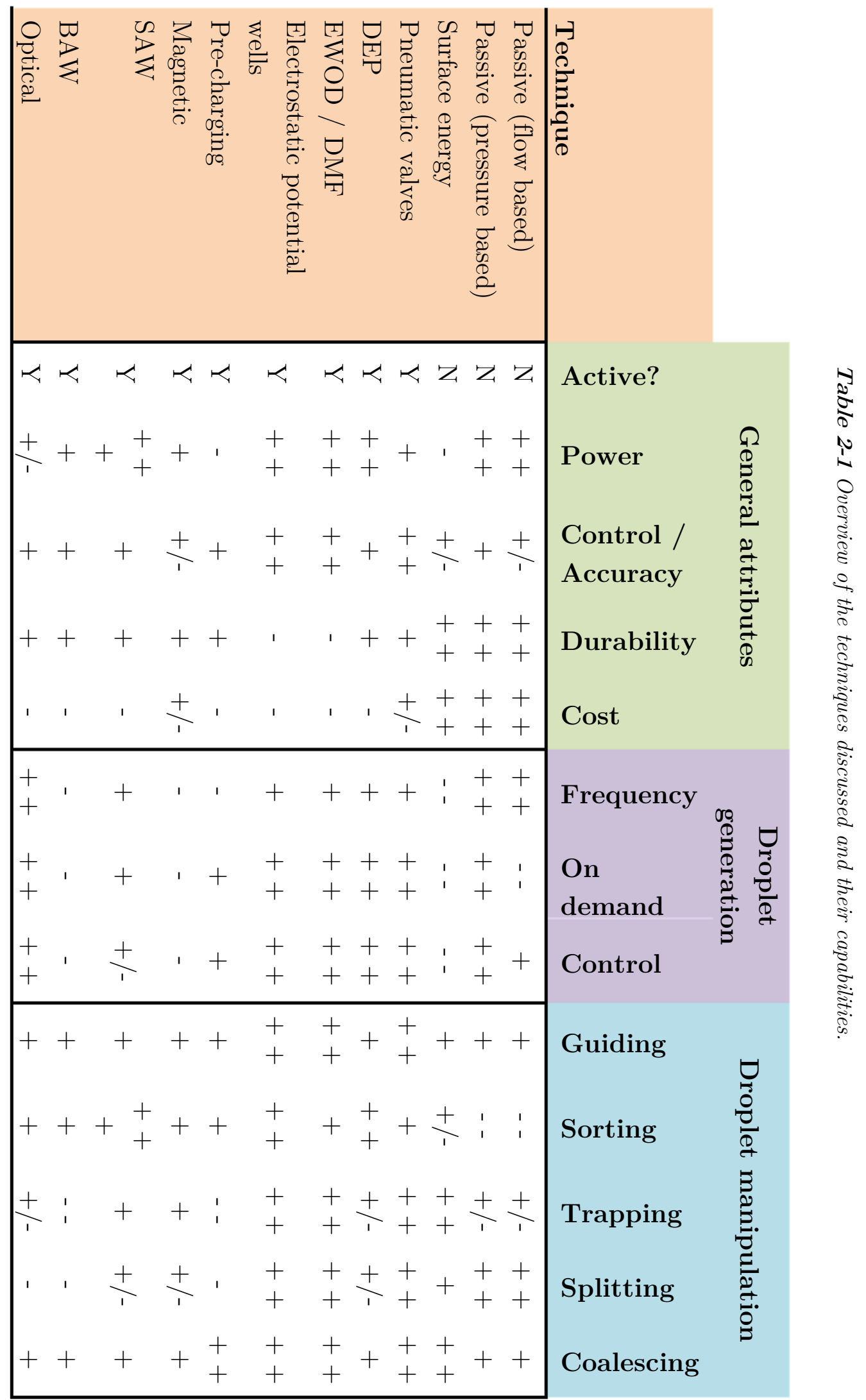




\section{References}

1. Whitesides, G.M., The origins and the future of microfluidics. Nature, 2006. 442(7101): p. 368-73.

2. Leman, M., F. Abouakil, A.D. Griffiths, et al., Droplet-based microfluidics at the femtolitre scale. Lab on a Chip, 2015. 15(3): p. 753-65.

3. Um, E., M.E. Rogers, and H.A. Stone, Combinatorial generation of droplets by controlled assembly and coalescence. Lab on a Chip, 2013. 13(23): p. 4674-80.

4. Link, D.R., S.L. Anna, D.A. Weitz, et al., Geometrically mediated breakup of drops in microfluidic devices. Phys Rev Lett, 2004. 92(5): p. 054503.

5. Fradet, E., C. McDougall, P. Abbyad, et al., Combining rails and anchors with laser forcing for selective manipulation within 2D droplet arrays. Lab on a Chip, 2011. 11(24): p. 4228-34.

6. Huebner, A., D. Bratton, G. Whyte, et al., Static microdroplet arrays: a microfluidic device for droplet trapping, incubation and release for enzymatic and cell-based assays. Lab on a Chip, 2009. 9(5): p. 692-8.

7. Bithi, S.S., W.S. Wang, M. Sun, et al., Coalescing drops in microfluidic parking networks: A multifunctional platform for drop-based microfluidics. Biomicrofluidics, 2014. 8(3): p. 034118.

8. Chen, J.S. and J.H. Jiang, Droplet Microfluidic Technology: Mirodroplets Formation and Manipulation. Chinese Journal of Analytical Chemistry, 2012. 40(8): p. 1293-300.

9. Gu, H., M.H.G. Duits, and F. Mugele, Droplets Formation and Merging in Two-Phase Flow Microfluidics. International Journal of Molecular Sciences, 2011. 12(4): p. 2572-97.

10. Baroud, C.N., F. Gallaire, and R. Dangla, Dynamics of microfluidic droplets. Lab on a Chip, 2010. 10(16): p. 2032-45.

11. Collins, D.J., A. Neild, A. deMello, et al., The Poisson distribution and beyond: methods for microfluidic droplet production and single cell encapsulation. Lab on a Chip, 2015. 15(17): p. 3439-59.

12. Casadevall i Solvas, X. and A. deMello, Droplet microfluidics: recent developments and future applications. Chemical Communications, 2011. 47(7): p. 1936-42.

13. Cho, S.K., H.J. Moon, and C.J. Kim, Creating, transporting, cutting, and merging liquid droplets by electrowetting-based actuation for digital microfluidic circuits. Journal of Microelectromechanical Systems, 2003. 12(1): p. 70-80.

14. Kaler, K. and R. Prakash, Droplet Microfluidics for Chip-Based Diagnostics. Sensors, 2014. 14(12): p. 23283-306.

15. Mugele, F., Fundamental challenges in electrowetting: from equilibrium shapes to contact angle saturation and drop dynamics. Soft Matter, 2009. 5(18): p. 3377-84.

16. Guo, M.T., A. Rotem, J.A. Heyman, et al., Droplet microfluidics for high-throughput biological assays. Lab on a Chip, 2012. 12(12): p. 2146-55.

17. Takinoue, M. and S. Takeuchi, Droplet microfluidics for the study of artificial cells. Analytical and Bioanalytical Chemistry, 2011. 400(6): p. 1705-16.

18. Theberge, A.B., F. Courtois, Y. Schaerli, et al., Microdroplets in Microfluidics: An Evolving Platform for Discoveries in Chemistry and Biology. Angewandte Chemie-International Edition, 2010. 49(34): p. 5846-68.

19. Baret, J.C., Surfactants in droplet-based microfluidics. Lab on a Chip, 2012. 12(3): p. 422-33. 
20. Seemann, R., M. Brinkmann, T. Pfohl, et al., Droplet based microfluidics. Reports on Progress in Physics, 2012. 75(1).

21. Song, H., D.L. Chen, and R.F. Ismagilov, Reactions in droplets in microfluidic channels. Angew Chem Int Ed Engl, 2006. 45(44): p. 7336-56.

22. Choi, K., A.H. Ng, R. Fobel, et al., Digital microfluidics. Annu Rev Anal Chem (Palo Alto Calif), 2012. 5: p. 413-40.

23. Day, P., A. Manz, and Y. Zhang, Microdroplet technology : principles and emerging applications in biology and chemisty. Intergrated analytical systems. 2012, New York: Springer. x, 243 pages.

24. Bruus, H., Theoretical microfluidics. Oxford master series in physics. 2008, Oxford ; New York: Oxford University Press. 346 p.

25. Vanapalli, S.A., A.G. Banpurkar, D. van den Ende, et al., Hydrodynamic resistance of single confined moving drops in rectangular microchannels. Lab on a Chip, 2009. 9(7): p. 982-90.

26. de Ruiter, R., A.M. Pit, V.M. de Oliveira, et al., Electrostatic potential wells for on-demand drop manipulation in microchannels. Lab Chip, 2014. 14(5): p. 883-91.

27. Jin, B.J., Y.W. Kim, Y. Lee, et al., Droplet merging in a straight microchannel using droplet size or viscosity difference. Journal of Micromechanics and Microengineering, 2010. 20(3).

28. Blom, M.T., E. Chmela, R.E. Oosterbroek, et al., On-chip hydrodynamic chromatography separation and detection of nanoparticles and biomolecules. Analytical Chemistry, 2003. 75(24): p. 6761-8.

29. Bithi, S.S. and S.A. Vanapalli, Collective dynamics of non-coalescing and coalescing droplets in microfluidic parking networks. Soft Matter, 2015. 11(25): p. 5122-32.

30. Korczyk, P.M., L. Derzsi, S. Jakiela, et al., Microfluidic traps for hard-wired operations on droplets. Lab on a Chip, 2013. 13(20): p. 4096-102.

31. Dangla, R., S. Lee, and C.N. Baroud, Trapping microfluidic drops in wells of surface energy. Phys Rev Lett, 2011. 107(12): p. 124501.

32. Amselem, G., P.T. Brun, F. Gallaire, et al., Breaking Anchored Droplets in a Microfluidic Hele-Shaw Cell. Physical Review Applied, 2015. 3(5): p. 054006.

33. Abbyad, P., R. Dangla, A. Alexandrou, et al., Rails and anchors: guiding and trapping droplet microreactors in two dimensions. Lab on a Chip, 2011. 11(5): p. 813-21.

34. Dangla, R., S.C. Kayi, and C.N. Baroud, Droplet microfluidics driven by gradients of confinement. Proceedings of the National Academy of Sciences of the United States of America, 2013. 110(3): p. 853-8.

35. Xu, L.F., H. Lee, R. Panchapakesan, et al., Fusion and sorting of two parallel trains of droplets using a railroad-like channel network and guiding tracks. Lab on a Chip, 2012. 12(20): p. 3936-42.

36. McDonald, J.C., D.C. Duffy, J.R. Anderson, et al., Fabrication of microfluidic systems in poly(dimethylsiloxane). Electrophoresis, 2000. 21(1): p. 27-40.

37. Duffy, D.C., J.C. McDonald, O.J. Schueller, et al., Rapid Prototyping of Microfluidic Systems in Poly(dimethylsiloxane). Anal Chem, 1998. 70(23): p. 4974-84.

38. Unger, M.A., H.P. Chou, T. Thorsen, et al., Monolithic microfabricated valves and pumps by multilayer soft lithography. Science, 2000. 288(5463): p. 113-6.

39. Abate, A.R., J.J. Agresti, and D.A. Weitz, Microfluidic sorting with high-speed single-layer membrane valves. Applied Physics Letters, 2010. 96(20). 
40. Lee, W.S., S. Jambovane, D. Kim, et al., Predictive model on micro droplet generation through mechanical cutting. Microfluidics and Nanofluidics, 2009. 7(3): p. 431-8.

41. Zeng, S.J., B.W. Li, X.O. Su, et al., Microvalve-actuated precise control of individual droplets in microfluidic devices. Lab on a Chip, 2009. 9(10): p. 1340-3.

42. Jambovane, S., D.J. Kim, E.C. Duin, et al., Creation of Stepwise Concentration Gradient in Picoliter Droplets for Parallel Reactions of Matrix Metalloproteinase II and IX. Analytical Chemistry, 2011. 83(9): p. 3358-64.

43. Leung, K., H. Zahn, T. Leaver, et al., A programmable droplet-based microfluidic device applied to multiparameter analysis of single microbes and microbial communities. Proc Natl Acad Sci U S A, 2012. 109(20): p. 7665-70.

44. Cetin, B. and D. Li, Dielectrophoresis in microfluidics technology. Electrophoresis, 2011. 32(18): p. 2410-27.

45. Pethig, R., Review article-dielectrophoresis: status of the theory, technology, and applications. Biomicrofluidics, 2010. 4(2).

46. Ahn, K., C. Kerbage, T.P. Hunt, et al., Dielectrophoretic manipulation of drops for high-speed microfluidic sorting devices. Applied Physics Letters, 2006. 88(2).

47. Mugele, F. and J.-C. Baret, Electrowetting: from basics to applications. Journal of Physics: Condensed Matter, 2005. 17(28): p. R705.

48. de Ruiter, R., P. Wennink, A.G. Banpurkar, et al., Use of electrowetting to measure dynamic interfacial tensions of a microdrop. Lab Chip, 2012. 12(16): p. 2832-6.

49. Baratian, D., A. Cavalli, D. van den Ende, et al., On the shape of a droplet in a wedge: new insight from electrowetting. Soft Matter, 2015. 11(39): p. 7717-21.

50. Jones, T.B., On the relationship of dielectrophoresis and electrowetting. Langmuir, 2002. 18(11): p. 4437-43.

51. Abdelgawad, M., S.L.S. Freire, H. Yang, et al., All-terrain droplet actuation. Lab on a Chip, 2008. 8(5): p. 672-7.

52. Bhattacharjee, B. and H. Najjaran, Droplet sensing by measuring the capacitance between coplanar electrodes in a digital microfluidic system. Lab on a Chip, 2012. 12(21): p. 4416-23.

53. Hayes, R.A. and B.J. Feenstra, Video-speed electronic paper based on electrowetting. Nature, 2003. 425(6956): p. 383-5.

54. Hadwen, B., G.R. Broder, D. Morganti, et al., Programmable large area digital microfluidic array with integrated droplet sensing for bioassays. Lab on a Chip, 2012. 12(18): p. 3305-13.

55. Banerjee, A.N., S.Z. Qian, and S.W. Joo, High-speed droplet actuation on single-plate electrode arrays. Journal of Colloid and Interface Science, 2011. 362(2): p. 567-74.

56. Caputo, D., G. de Cesare, N. Lo Vecchio, et al., Polydimethylsiloxane material as hydrophobic and insulating layer in electrowetting-on-dielectric systems. Microelectronics Journal, 2014. 45(12): p. 1684-90.

57. Fobel, R., C. Fobel, and A.R. Wheeler, DropBot: An open-source digital microfluidic control system with precise control of electrostatic driving force and instantaneous drop velocity measurement. Applied Physics Letters, 2013. 102(19).

58. Pit, A.M., R. de Ruiter, A. Kumar, et al., High-throughput sorting of drops in microfluidic chips using electric capacitance. Biomicrofluidics, 2015. 9(4): p. 044116.

59. Link, D.R., E. Grasland-Mongrain, A. Duri, et al., Electric control of droplets in microfluidic devices. Angewandte Chemie-International Edition, 2006. 45(16): p. 2556-60. 
60. Ahn, B., K. Lee, R. Panchapakesan, et al., On-demand electrostatic droplet charging and sorting. Biomicrofluidics, 2011. 5(2): p. 24113.

61. Rao, L., B. Cai, J.L. Wang, et al., A microfluidic electrostatic separator based on pre-charged droplets. Sensors and Actuators B-Chemical, 2015. 210: p. 328-35.

62. Ali-Cherif, A., S. Begolo, S. Descroix, et al., Programmable Magnetic Tweezers and Droplet Microfluidic Device for High-Throughput Nanoliter Multi-Step Assays. Angewandte ChemieInternational Edition, 2012. 51(43): p. 10765-9.

63. Teste, B., A. Ali-Cherif, J.L. Viovy, et al., A low cost and high throughput magnetic beadbased immuno-agglutination assay in confined droplets. Lab on a Chip, 2013. 13(12): p. 23449 .

64. Teste, B., N. Jamond, D. Ferraro, et al., Selective handling of droplets in a microfluidic device using magnetic rails. Microfluidics and Nanofluidics, 2015. 19(1): p. 141-53.

65. Katsikis, G., J.S. Cybulski, and M. Prakash, Synchronous universal droplet logic and control. Nat Phys, 2015. 11(7): p. 588-96.

66. White, R.M. and F.W. Voltmer, Direct Piezoelectric Coupling to Surface Elastic Waves. Applied Physics Letters, 1965. 7(12): p. 314-\&.

67. Shi, J.J., X.L. Mao, D. Ahmed, et al., Focusing microparticles in a microfluidic channel with standing surface acoustic waves (SSAW). Lab on a Chip, 2008. 8(2): p. 221-3.

68. Franke, T., A.R. Abate, D.A. Weitz, et al., Surface acoustic wave (SAW) directed droplet flow in microfluidics for PDMS devices. Lab Chip, 2009. 9(18): p. 2625-7.

69. Sesen, M., T. Alan, and A. Neild, Microfluidic plug steering using surface acoustic waves. Lab on a Chip, 2015. 15(14): p. 3030-8.

70. Sesen, M., T. Alan, and A. Neild, Microfluidic on-demand droplet merging using surface acoustic waves. Lab on a Chip, 2014. 14(17): p. 3325-33.

71. Collignon, S., J. Friend, and L. Yeo, Planar microfluidic drop splitting and merging. Lab on a Chip, 2015. 15(8): p. 1942-51.

72. Schmid, L., D.A. Weitz, and T. Franke, Sorting drops and cells with acoustics: acoustic microfluidic fluorescence-activated cell sorter. Lab on a Chip, 2014. 14(19): p. 3710-8.

73. Oever, J.v.t., R. Frentrop, D. Wijnperlé, et al., Imaging local acoustic pressure in microchannels. Applied Optics, 2015. 54(21): p. 6482-90.

74. Cheung, Y.N., N.T. Nguyen, and T.N. Wong, Droplet manipulation in a microfluidic chamber with acoustic radiation pressure and acoustic streaming. Soft Matter, 2014. 10(40): p. 812232 .

75. Leibacher, I., P. Reichert, and J. Dual, Microfluidic droplet handling by bulk acoustic wave (BAW) acoustophoresis. Lab Chip, 2015. 15(13): p. 2896-905.

76. Ashkin, A., J.M. Dziedzic, J.E. Bjorkholm, et al., Observation of a single-beam gradient force optical trap for dielectric particles. Opt Lett, 1986. 11(5): p. 288.

77. Sanders, J.L., Y.M. Yang, M.R. Dickinson, et al., Pushing, pulling and twisting liquid crystal systems: exploring new directions with laser manipulation. Philosophical Transactions of the Royal Society a-Mathematical Physical and Engineering Sciences, 2013. 371(1988).

78. Curtis, J.E., B.A. Koss, and D.G. Grier, Dynamic holographic optical tweezers. Optics Communications, 2002. 207(1-6): p. 169-75.

79. Zhao, Y.Q., G. Milne, J.S. Edgar, et al., Quantitative force mapping of an optical vortex trap. Applied Physics Letters, 2008. 92(16). 
80. Jung, J.H., K.H. Lee, K.S. Lee, et al., Optical separation of droplets on a microfluidic platform. Microfluidics and Nanofluidics, 2014. 16(4): p. 635-44.

81. Baroud, C.N., M.R. de Saint Vincent, and J.-P. Delville, An optical toolbox for total control of droplet microfluidics. Lab on a Chip, 2007. 7(8): p. 1029-33.

82. Park, S.-Y., T.-H. Wu, Y. Chen, et al., High-speed droplet generation on demand driven by pulse laser-induced cavitation. Lab on a Chip, 2011. 11(6): p. 1010-2.

83. Thorsen, T., R.W. Roberts, F.H. Arnold, et al., Dynamic pattern formation in a vesiclegenerating microfluidic device. Phys Rev Lett, 2001. 86(18): p. 4163-6.

84. Li, X.B., F.C. Li, J.C. Yang, et al., Study on the mechanism of droplet formation in T-junction microchannel. Chemical Engineering Science, 2012. 69(1): p. 340-51.

85. Anna, S.L., N. Bontoux, and H.A. Stone, Formation of dispersions using "flow focusing" in microchannels. Applied Physics Letters, 2003. 82(3): p. 364-6.

86. Cramer, C., P. Fischer, and E.J. Windhab, Drop formation in a co-flowing ambient fluid. Chemical Engineering Science, 2004. 59(15): p. 3045-58.

87. Guillot, P., A. Colin, A.S. Utada, et al., Stability of a jet in confined pressure-driven biphasic flows at low reynolds numbers. Physical Review Letters, 2007. 99(10).

88. Utada, A.S., L.Y. Chu, A. Fernandez-Nieves, et al., Dripping, jetting, drops, and wetting: The magic of microfluidics. Mrs Bulletin, 2007. 32(9): p. 702-8.

89. Mittal, N., C. Cohen, J. Bibette, et al., Dynamics of step-emulsification: From a single to a collection of emulsion droplet generators. Physics of Fluids, 2014. 26(8).

90. Li, Z., A.M. Leshansky, L.M. Pismen, et al., Step-emulsification in a microfluidic device. Lab on a Chip, 2015. 15(4): p. 1023-31.

91. Dangla, R., E. Fradet, Y. Lopez, et al., The physical mechanisms of step emulsification. Journal of Physics D-Applied Physics, 2013. 46(11).

92. Tan, S.H., F. Maes, B. Semin, et al., The Microfluidic Jukebox. Scientific Reports, 2014. 4.

93. Gu, H., C.U. Murade, M.H. Duits, et al., A microfluidic platform for on-demand formation and merging of microdroplets using electric control. Biomicrofluidics, 2011. 5(1): p. 11101.

94. Pollack, M.G., A.D. Shenderov, and R.B. Fair, Electrowetting-based actuation of droplets for integrated microfluidics. Lab on a Chip, 2002. 2(2): p. 96-101.

95. Collins, D.J., T. Alan, K. Helmerson, et al., Surface acoustic waves for on-demand production of picoliter droplets and particle encapsulation. Lab on a Chip, 2013. 13(16): p. 3225-31.

96. Lin, R., J.S. Fisher, M.G. Simon, et al., Novel on-demand droplet generation for selective fluid sample extraction. Biomicrofluidics, 2012. 6(2).

97. Lin, B.C. and Y.C. Su, On-demand liquid-in-liquid droplet metering and fusion utilizing pneumatically actuated membrane valves. Journal of Micromechanics and Microengineering, 2008. 18(11).

98. Li, S.X., X.Y. Ding, F. Guo, et al., An On-Chip, Multichannel Droplet Sorter Using Standing Surface Acoustic Waves. Analytical Chemistry, 2013. 85(11): p. 5468-74.

99. Baret, J.C., O.J. Miller, V. Taly, et al., Fluorescence-activated droplet sorting (FADS): efficient microfluidic cell sorting based on enzymatic activity. Lab on a Chip, 2009. 9(13): p. 1850-8.

100. Thomas, R.S., H. Morgan, and N.G. Green, Negative DEP traps for single cell immobilisation. Lab on a Chip, 2009. 9(11): p. 1534-40.

101. Pollack, M.G., R.B. Fair, and A.D. Shenderov, Electrowetting-based actuation of liquid 
droplets for microfluidic applications. Applied Physics Letters, 2000. 77(11): p. 1725-6.

102. Bremond, N., A.R. Thiam, and J. Bibette, Decompressing emulsion droplets favors coalescence. Phys Rev Lett, 2008. 100(2): p. 024501.

103. Jung, J.H., K.H. Lee, G. Destgeer, et al., In situ seriate droplet coalescence under an optical force. Microfluidics and Nanofluidics, 2015. 18(5-6): p. 1247-54.

104. Lorenz, R.M., J.S. Edgar, G.D.M. Jeffries, et al., Vortex-trap-induced fusion of femtolitervolume aqueous droplets. Analytical Chemistry, 2007. 79(1): p. 224-8.

105. Follana, R., D. Klein, M.P. Krafft, et al., Prolonged shelf stability and biocompatibility of a concentrated injectable fluorocarbon emulsion. Biomater Artif Cells Immobilization Biotechnol, 1992. 20(2-4): p. 1059-61.

106. Akartuna, I., D.M. Aubrecht, T.E. Kodger, et al., Chemically induced coalescence in dropletbased microfluidics. Lab on a Chip, 2015. 15(4): p. 1140-4.

107. Schoeman, R.M., E.W. Kemna, F. Wolbers, et al., High-throughput deterministic single-cell encapsulation and droplet pairing, fusion, and shrinkage in a single microfluidic device. Electrophoresis, 2014. 35(2-3): p. 385-92.

108. Devaraju, N.S.G.K. and M.A. Unger, Pressure driven digital logic in PDMS based microfluidic devices fabricated by multilayer soft lithography. Lab on a Chip, 2012. 12(22): p. 4809-15.

109. Prakash, M. and N. Gershenfeld, Microfluidic bubble logic. Science, 2007. 315(5813): p. 8325.

110. Zagnoni, M. and J.M. Cooper, A microdroplet-based shift register. Lab on a Chip, 2010. 10(22): p. 3069-73.

111. Schlicht, B. and M. Zagnoni, Droplet-interface-bilayer assays in microfluidic passive networks. Scientific Reports, 2015. 5.

112. Tice, J.D., H. Song, A.D. Lyon, et al., Formation of droplets and mixing in multiphase microfluidics at low values of the Reynolds and the capillary numbers. Langmuir, 2003. 19(22): p. $9127-33$.

113. Brouzes, E., M. Medkova, N. Savenelli, et al., Droplet microfluidic technology for single-cell high-throughput screening. Proceedings of the National Academy of Sciences of the United States of America, 2009. 106(34): p. 14195-200.

114. Song, H., J.D. Tice, and R.F. Ismagilov, A microfluidic system for controlling reaction networks in time. Angewandte Chemie-International Edition, 2003. 42(7): p. 768-72.

115. Paik, P., V.K. Pamula, and R.B. Fair, Rapid droplet mixers for digital microfluidic systems. Lab on a Chip, 2003. 3(4): p. 253-9.

116. Mampallil, D., D. van den Ende, and F. Mugele, Controlling flow patterns in oscillating sessile drops by breaking azimuthal symmetry. Applied Physics Letters, 2011. 99(15).

117. Ng, A.H.C., K. Choi, R.P. Luoma, et al., Digital Microfluidic Magnetic Separation for Particle-Based Immunoassays. Analytical Chemistry, 2012. 84(20): p. 8805-12.

118. Jin, S.H., H.H. Jeong, B. Lee, et al., A programmable microfluidic static droplet array for droplet generation, transportation, fusion, storage, and retrieval. Lab on a Chip, 2015. 15(18): p. $3677-86$. 


\section{Electrostatic potential wells}

Precise control and manipulation of individual drops are crucial in many lab-ona-chip applications, e.g. as motivated in Chapter 1.1 to control and diagnose cellcontaining droplets. In this chapter we present a novel hybrid concept for channelbased discrete microfluidics with integrated electrowetting functionality by incorporating co-planar electrodes (separated by a narrow gap) in the microchannel substrate. By combining the high throughput of channel-based two phase flow microfluidics with the individual drop control achieved using electrical actuation, we acquire the strengths of both worlds. The tunable strength of the electrostatic forces enables a wide range of drop manipulations, such as ondemand trapping and release, guiding, and sorting of drops in the microchannel. In each of these scenarios, the retaining electrostatic force competes with the hydrodynamic drag force. The conditions for trapping can be predicted using a simple model that balances these forces.

This chapter has been adapted from the publication:

R. de Ruiter, A.M. Pit, V. Martins de Oliveira, M.H.G. Duits, H.T.M. van den Ende and F. Mugele, "Electrostatic potential wells for on-demand drop manipulation in microchannels", Lab Chip 14, 883-91 (2014) 


\subsection{Introduction}

In various fields in (cell) biology and chemistry, microfluidic drops are currently used as microreactors of picoliter to nanoliter volume with an isolated and controlled content. ${ }^{1,2}$ By injecting the analyte fluid into T-junctions, ${ }^{3,4}$ or flow focusing devices, ${ }^{5}$ or using microchannel step emulsification, ${ }^{6-8}$ monodispersed liquid drops can be generated up to $k H z$ frequencies in channel-based discrete microfluidics. However, applications such as high-throughput screening of cells and analysis of chemical reactions require in addition high-speed manipulation and individual control over specific drops.

In flow or pressure driven microfluidics drops are often manipulated passively via topological modification of the channels, e.g. by using variations in the channel dimensions to modify the hydrodynamic resistance of different paths, or capillary valves to modify the pressure required for drop transport. Several drop manipulations have been demonstrated, such as transport, trapping, sorting, merging, and splitting (See also Chapter 2). ${ }^{1,9-11}$ An illustrative example is the trapping of drops in storage wells connected to the main channel, making use of a combination of capillary forces and differences in hydrodynamic resistance. An alternative flow path is present for the continuous phase in the form of bypass channels when the wells are occupied. ${ }^{12}, 13$ On the other hand, drops can also be trapped at specific locations in the main channel itself. Small holes have been introduced in the top wall of a wide and thin microchannel to function as anchors. Confined drops that are passing by expand into the hole to decrease their surface area and interfacial energy, generating an anchoring force that can hold the drop against the drag force of the surrounding flow. ${ }^{14,15}$ The merging of subsequent drops has been achieved for example by widening the channel ${ }^{16}$ or capturing drops in between rows of pillars, ${ }^{17}$ and splitting is accomplished in T-junctions or by introducing isolated obstacles in the flow. ${ }^{18-}$ ${ }^{20}$ Since these passive manipulations are in general non-selective and not (easily) tunable, precise control over individual drops is limited. For example, a drop trapped in a storage well or by a small geometric defect in the microchannel wall cannot be easily released by changing the trapping force. To remove the drop from the trap, a change in the direction or magnitude of the flow is required. ${ }^{12-15}$ Another possible disadvantage is that if arrays of drops are trapped, they will all be released simultaneously. A combination of active methods, e.g. laser forcing ${ }^{21}$ or dielectrophoresis ${ }^{22}$ is necessary to release single drops.

Precise control over individual drops can be achieved via electrowetting-based digital microfluidics (DMF), in which units of nanoliter to microliter volume are actuated using a series of adjacent electrodes. ${ }^{23-27}$ The same types of drop manipulation as described above are then performed via switching of individually addressable electrodes that change the local wettability. In DMF the electrodes provide the actuation, no driving flow is used. Devices are either based on a parallel plate set-up, in which drops are sandwiched between a substrate with insulated actuation electrodes and a grounded top plate, or on co-planar designs, where both the actuation and ground electrodes are located on the same substrate. Although high actuation speeds can be achieved in DMF, the throughput is always lower than in channel-based microfluidics and subnanoliter volumes are difficult to achieve. In addition, higher throughputs require extensive programming of a large number of electrodes.

In this chapter we combine the high throughput and low sample volumes used in channel- 
based microfluidics and the individual drop control achieved using electrical actuation by incorporating insulator-covered electrodes in the walls of micro-channels. The isolation of the electrodes from the aqueous drops offers clear advantages over designs in which there is direct contact between electrodes and fluid, ${ }^{28,29}$ as it prevents electrochemical reactions that could be detrimental to various (e.g. biological) samples. Electrodes can be positioned at various locations along the channel in several configurations to perform different drop manipulations, thereby fully integrating the two techniques spatially. This approach differs from a previous hybrid design where a co-planar digital microfluidics (DMF) unit was placed on the front end of a microchannel based continuous flow system ${ }^{30}$ and DMF was used to pre-process liquid drops. Then a small fraction was loaded into the channel for single phase chemical separations.

The voltages required for the electrical actuation of drops depend on the thickness of the dielectric layer, but they are generally much lower than the kilovolts used in dielectrophoretic (DEP) actuation, where polarizable drops are manipulated with nonuniform electric fields. ${ }^{22,31-33}$ In addition, electrical actuation offers a more direct and local control, allowing for drop trapping above the electrodes inside the main microchannel.

In previous work by our group, the combination of microfluidics and electrowetting (EW) was used to control the size and frequency of drop formation. ${ }^{34,35}$ We still incorporate this system in our microchannel, but also add a new feature: we exploit electrostatic potential wells to manipulate drops in a microchannel flow. Several operations can be implemented, such as on-demand trapping and release, which is crucial for the storage of drops that need to be analyzed in situ for some time. To create a trapping site we use two co-planar electrodes $^{36}$ separated by a narrow gap. A co-planar design allows for easy fabrication of the microfluidic device, as both the actuation and ground electrodes are situated on one of the channel substrates. In more complex lab-on-a-chip devices co-planar electrodes in one substrate can be combined with another functional technique in the vacant substrate, of which an example is researched in Chapter 4. For example, drops with cells can be trapped in specific positions where ligands are patterned in the opposing channel wall, allowing for the analysis of cell-excreted proteins using Surface Plasmon Resonance $(\mathrm{SPR})^{37}$ or other techniques.

When a conductive droplet is positioned above both insulator-covered electrodes, a series of two parallel-plate capacitors is formed, which generates an electrical potential well that centers the drop above the two electrodes. The associated attractive force is proportional to the square of the applied voltage, allowing for a tunable trapping strength. ${ }^{38}$ The equilibrium position of the drop (with respect to the trap) is governed by a force balance between the retaining and driving forces. The latter is the hydrodynamic drag force exerted by the continuous phase flow, which is adjustable via the flow rate. The trapping and driving forces are thus independently adjustable. In Section 3.3.1 we discuss the principle and modelling of the electrostatic trap and compare the trapping and driving forces under various experimental conditions. The versatility of our approach for several applications in lab-on-a-chip devices is illustrated in Section 3.3.2. We demonstrate the guiding and sorting of drops, offering a simple alternative to sorting by dielectrophoresis ${ }^{31}$, ${ }^{33}$ or surface acoustic waves. ${ }^{39}$ We also create arrays of electrode traps to stop and release multiple droplets. 


\subsection{Materials and methods}

The microchannels were fabricated from polydimethylsiloxane (PDMS, Sylgard 184) using standard soft lithography. ${ }^{40}$ Fig. 3 -1a gives an overview of the device, which consists of a flow focusing device (FFD) with a tapered water inlet where aqueous drops are formed and a main channel containing the electrodes. The height $h$ of the device is $50 \mu \mathrm{m}$, and the width $w$ of the main channel is $3 \mathrm{~mm}$. Due to the extreme aspect ratio the main channel can be considered as a Hele-Shaw cell. ${ }^{41}$

The bottom substrate is fabricated from indium tin oxide (ITO) coated glass substrates. Patterned electrodes are obtained via photolithography and etching in a solution of $18 \%$ $\mathrm{HCl}$, creating gaps between the electrodes that are $10 \mu \mathrm{m}$ wide. The substrate is spin coated with PDMS at $6000 \mathrm{rpm}$ for $600 \mathrm{~s}$ to obtain an approximately $3 \mu \mathrm{m}$ thick insulating layer that separates the aqueous drops from the electrode. While other insulating materials are generally preferable in EW, the use of PDMS was found to provide the most robust devices in terms of channel-to-substrate adhesion and leak-tightness. The
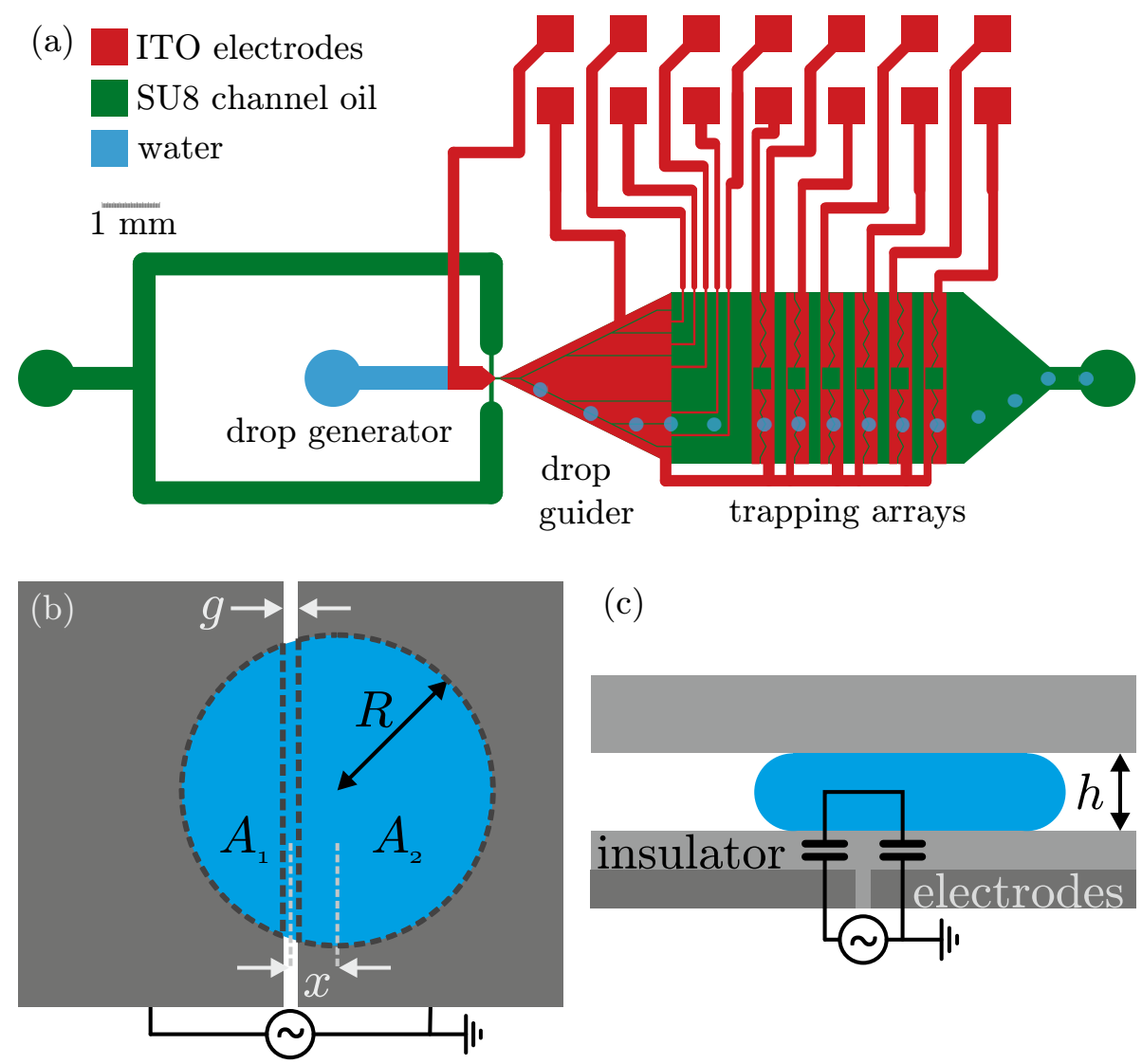

(c)

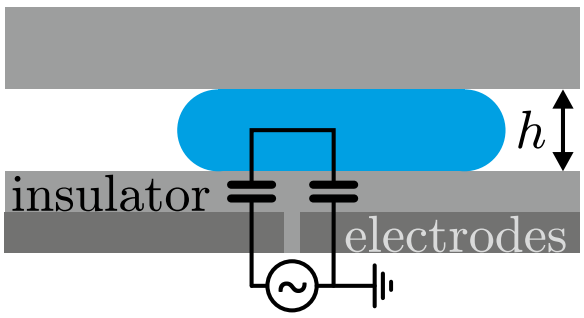

Fig. 3-1 Device geometry and generated electrostatic potential well. (a) Overview of one of the devices showing a drop formation unit and a main channel with guiding and trapping electrodes. (b) Top view and (c) side view of the co-planar electrode design. 
PDMS layer is partially cured at $75{ }^{\circ} \mathrm{C}$ for $20 \mathrm{~min}$ and subsequently connected to the microchannels. An alignment microscope is used to ensure the electrodes are aligned in the main channel. Hereafter, the curing is completed at $75^{\circ} \mathrm{C}$ for an additional 40 minutes. The properties of the dielectric layer are measured from the electrowetting response, i.e. the voltage-dependent $\cos \theta=\cos \theta_{Y}+\eta$ on a simultaneously fabricated evenly covered flat substrate. Here, $\theta_{Y}$ is the Young angle, and $\eta=\varepsilon_{0} \varepsilon_{d} U^{2} /(2 d \gamma)$ is the electrowetting number, ${ }^{23}$ where $\varepsilon_{0} \varepsilon_{d}$ is the dielectric permittivity of the substrate with thickness $d, U$ is the applied root mean square (RMS) voltage, and $\gamma$ is the interfacial tension of the oil/water interface. Using this method, the capacitance per area $c=\varepsilon_{0} \varepsilon_{d} / d$ of the dielectric layer is determined to be $\sim 5.8 \mu \mathrm{F} \mathrm{m} \mathrm{m}^{-2}$. The effective electrowetting number in the experiments is generally $<1$.

Mineral oil (viscosity $\mu \approx 31 \mathrm{mPa} s$ ) is used as the continuous phase, and the dispersed phase consists of de-ionized water (Millipore Synergy UV, $18.2 \mathrm{M} \Omega \mathrm{cm}$ ) with $\mathrm{KCl}$ added up to a conductivity $\sigma$ of $3 \mathrm{mS} \mathrm{cm}^{-1}(\sim 25 \mathrm{mM})$. The interfacial tension of the water/oil interface $\gamma$ is $50 \mathrm{mNm}^{-1}$, and the advancing and receding water in oil contact angles on PDMS are determined to be $148^{\circ}$ and $138^{\circ}$, respectively.

Continuous oil flows are driven by using a syringe pump. The dispersed water phase is brought to the flow focusing device (FFD) by a secondary syringe pump or by adjusting the water pressure. The required pressure depends on the applied oil flow rate and the hydraulic resistance of the channels. Drops are thus either formed continuously by application of a continuous flow rate, or they are formed on demand by generating short air-pressure pulses using a pressure regulator (Parker Hannifin Corp.), a three-way solenoid valve (Takasago Electric Inc.), and a solid state relay (International Rectifier) which are controlled using home-made circuitry, a data acquisition (DAq) card (National Instruments), and a custom-written LabView program. The air pressure can be regulated from about 1-100 $\mathrm{kPa}$. There is also another mechanism for drop generation: application of an $\mathrm{AC}$ voltage to an electrode below the water phase. More information on droplet generation is described in Section 3.3.2.1.

To generate electrostatic forces on the drops, an alternating (AC) voltage (frequency: 1 $k H z$, RMS voltage: $0-350 \mathrm{~V}$ ) is applied between the active and grounded electrodes in the main channel. The actuation of multiple electrodes is accomplished by a home-made switchbox containing solid state relay switches (LH1502BAC, Vishay). The switches are controlled by TTL pulses using a LabVIEW program and DAq card. The device is observed using an inverted microscope (TI-U, Nikon), and the behavior of the generated drops is recorded using a high speed camera (FASTCAM SA5, Photron).

\subsection{Results}

\subsubsection{Principles of on-demand trapping and release}

\subsubsection{Electrostatic potential wells}

We first discuss the potential wells as generated by two co-planar electrodes separated by a gap. This case has already been described by 't Mannetje, et al. $(2013)^{38}$ for sphericalcap shaped drops resting on an open surface. In our case, the drops are confined between 
the bottom and top walls of a microchannel, where the radii of the drops in the horizontal plane are larger than the height of the channel $(R>h)$. Nevertheless, the underlying physical principles are the same for these two traps.

Briefly, when a confined drop of an electrically conductive liquid 'touches' (i.e. capacitive couples to) both electrodes, an electrical circuit containing two parallel plate capacitors in series is formed (Fig. 3-1c). For the salt concentrations and AC frequencies considered in our experiments, the liquid can be considered as a perfect conductor (i.e. surface charges completely screen the interior of the drop from the electric field). ${ }^{42}$ The capacitance between the drop and the electrodes is dominated by the area of the drop-substrate interface with negligible contributions from the contact line. Restricting our analysis to confined water drops with $R>h$, the contribution of oil intervening between the drop and the solid wall is reduced to a thin film that is negligible compared to the much thicker dielectric layers. ${ }^{43}$ We note, however, that our device is also capable of trapping drops with $R<h$ - albeit with somewhat reduced trapping forces as compared to the analysis presented below.

The overall capacitance is $C(x)=C_{1}(x) C_{2}(x) /\left(C_{1}(x)+C_{2}(x)\right)$, where the individual capacitances are given by $C_{1,2}(x)=\left(\varepsilon_{0} \varepsilon_{d} / d\right) A_{1,2}(x)$. Here, $A_{1,2}(x)$ is the area of the squeezed drop above the respective electrode. The areas depend on the position $x$ of the center of mass of the drop (see Fig. 3-1b). Charging the capacitors by applying a voltage generates an electrostatic free-energy landscape $W_{e}(x)=-C(x) U^{2} / 2=-c A U^{2} f(x)$, where $A=\pi R^{2}$ is the total contact area of the drop. The dimensionless function $f(x)$ can be derived from geometry and accounts for the variations in the areas. In Fig. 3-2a the black line shows $f(x)$ for the perpendicular gap electrode geometry. For this geometry the energy landscape it is shaped as a potential well.

The corresponding trapping force $F_{e}(x)=-\mathrm{d} W_{e}(x) / \mathrm{d} x$ has a maximum value $F_{e, \text { max }}=$ $-a c R U^{2}$. We thus find that the maximum trapping force scales with the capacitance per area, the radius of the (squeezed) drop, and the square of the voltage, with a position dependent proportionality constant $a$ of order unity that depends on the electrode configuration. In Fig. 3-2a the dashed red line shows $a$ for the perpendicular gap electrode geometry. As can be seen, the electric force will always be oriented towards the gap.

As we can see from Fig. 3-2a, for a gap between two (effectively) infinite electrodes, the electrostatic energy is minimal when the drop is centered above the gap. For a negligible gap width $(g \ll R)$, the trapping force reaches a maximum $F_{e, \max }= \pm 0.58 c R U^{2}$ at $x / R \approx \pm 0.67$. In a microchannel with the gap oriented perpendicular to the flow direction, the hydrodynamic forces will tend to push the drop through the potential well, leading to a counteracting force in the downstream half of the potential and the possibility of drop trapping between $x / R=0$ and $x / R \approx 0.67$. If the drag force exceeds the maximum value of the electrostatic force, the drop will pass the trap. In case the gap runs parallel to the flow direction in between two finite electrodes (See center of Fig. 3-2b), the hydrodynamic forces will push the drop towards the terminal at the edge of the electrodes. The areas of the drop above each electrode, which are always equal due to the centering effect of energy minimization, decrease as the drop is passing the trap. The electrostatic energy is now minimum if the drop is still completely in contact with the electrodes, and increases continuously afterwards. The trapping force reaches its 
maximum $F_{e l, \max }=-1 / 4 c R U^{2}$ at $x / R=0$, i.e. when the drop is halfway down the edge of the electrodes (note that this maximum force is $57 \%$ lower as compared to the perpendicular gap).

In this simplified analysis the voltage-dependent change in contact angle and the resulting changes in the drop shape are neglected. Estimates show an overestimation of $F_{e, \max }$ of maximum $20 \%$ for most experiments. More accurate values for the trapping force are obtained by taking into account the finite gap width between the electrodes. A gap width $g$ introduces a correction factor of $(1-g / 2 R)$ to the trapping force, which for our experiments with $10 \mu \mathrm{m}$ gaps and drops of $R>50 \mu \mathrm{m}$ is always larger than 0.85 . Drop deformations during trapping are neglected.

(a)

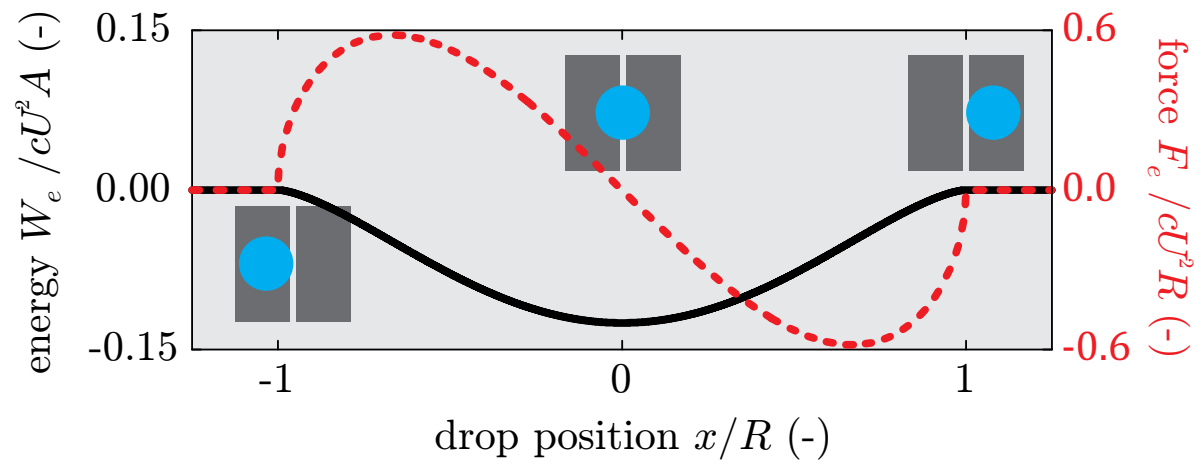

(b)
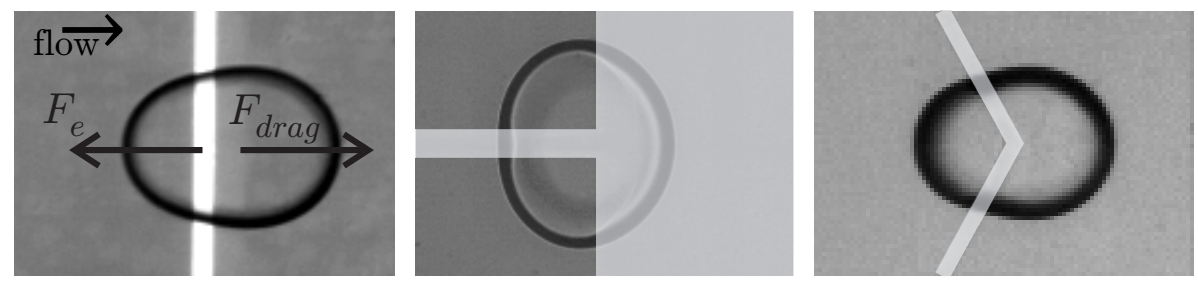

Fig. 3-2 Drop trapping in electrostatic potential wells. (a) The electrostatic contribution to the free energy of the system (black solid line) and the resulting electrostatic force (red dashed line) for two co-planar electrodes separated by a narrow gap $(g \rightarrow 0)$ oriented perpendicular to the flow direction. (b) Examples of drop trapping with various co-planar electrode designs: perpendicular gap, parallel gap, and V-shaped gap. The gaps between the electrodes are indicated in white.

Fig. 3-2b shows drops that are trapped using different configurations: perpendicular (left) and parallel gaps (middle) and a V-shaped gap (right). The choice of electrode configuration should be made in relation to the desired application. While the perpendicular gap yields the largest trapping force, the drops are free to move along the gap after trapping. In contrast, the parallel gap has a smaller trapping strength but has 
the benefits of keeping the drops on a predefined track in the microchannel and confining the trapped drop to a specific position. The V-shaped gap directs the drop to the middle of the trap upon trapping, combining the strong force from the perpendicular trap with the predetermined trapping location of the parallel gap.
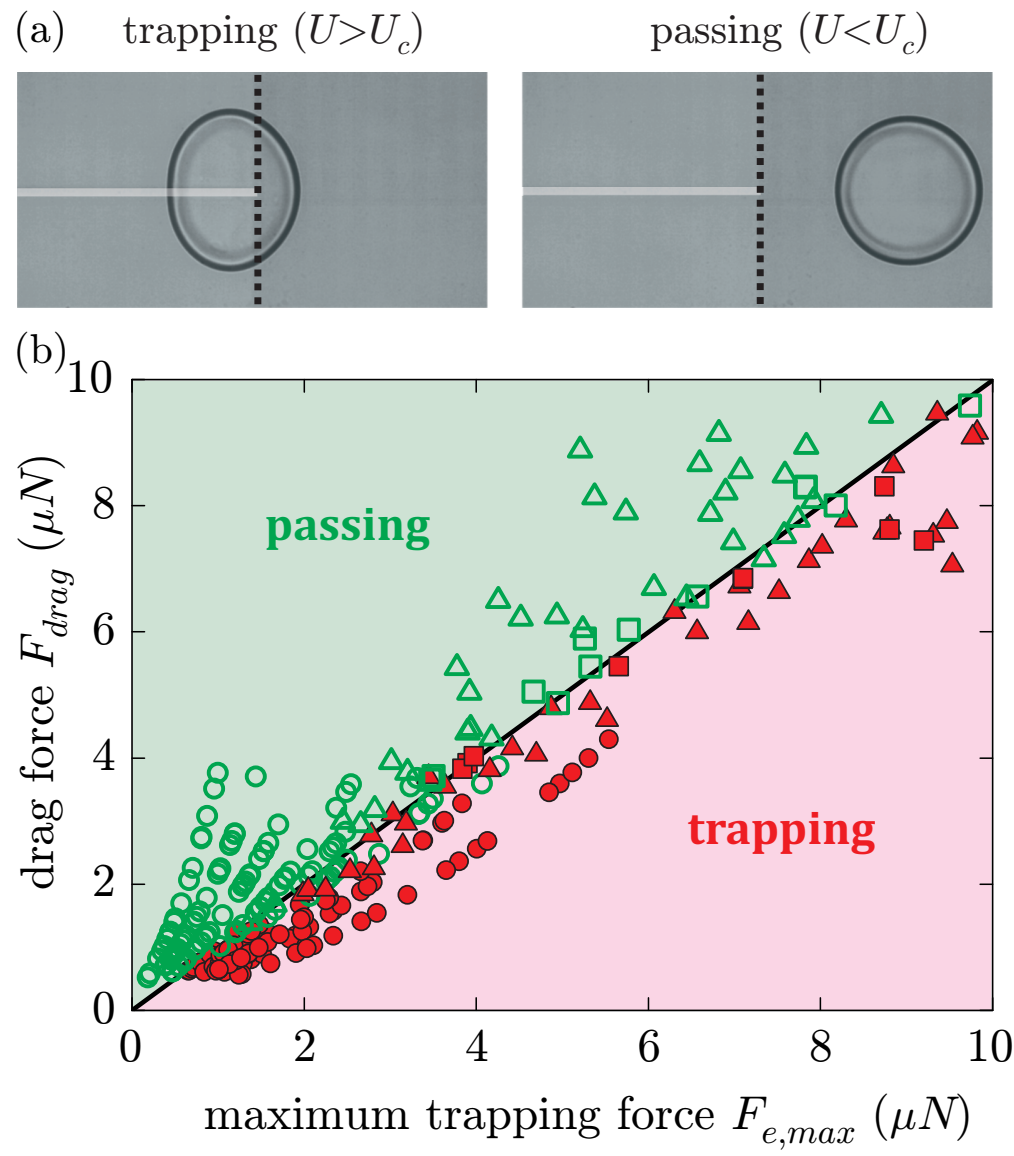

Fig. 3-3 (a) Images showing droplets of similar radius being trapped or not trapped depending on applied voltage. (b) Drag force versus maximum trapping force for the experimental results using different variables $u=0.8 \mathrm{~mm} \mathrm{~s}^{-1}$ (circles), $u=2.5 \mathrm{~mm} \mathrm{~s}^{-1}$ (triangles) with variable $R$, and at different flow velocities for a set $R=125 \mu \mathrm{m}$ (squares)). The transition from passing (green open symbols) to trapping (red closed symbols) occurs when both forces are equal.

\subsubsection{Hydrodynamic drag}

To predict the trapping of droplets we don't only need to consider the maximum electrostatic trapping force, but also how it balances with the hydrodynamic driving force. The hydrodynamic drag force exerted by the ambient flow is obtained from the Stokes 
equation. Since $h \ll w$ the microchannel can be modelled as a Hele-Shaw cell. An analytical calculation of the drag force on a cylinder with no-slip boundary condition in an infinitely wide channel and where $R \gg h$ yields $F_{d r a g}=24 \pi \mu u R^{2} / h$. For $R \approx h$ the viscous drag needs to be considered as well. ${ }^{44}$

When the drop size becomes of the order of the channel width $w$, the droplet blocks a significant fraction of the channel width. This results in a drastic increase of the pressure difference along the drop, resulting in a diverging drag force. More details about these calculations are found in the study of Vanapalli, et al. (2009) ${ }^{45}$ and the Supplementary Information of de Ruiter, et al. $(2014)^{44}$

The analytic expressions show that the electric force scales with $R$ and $U^{2}$, while the hydrodynamic drag force scales with $R^{2}$. This means that the critical voltage $U_{c}$ required for trapping a droplet will increase with $R$. This in turn means that the electric trap is size-selective; for each given voltage we find a critical drop radius $R_{c}$ above which the drop cannot be trapped anymore. This relation is more clearly shown in Chapter 4, Section 4.5.1.

\subsubsection{Force balance}

Given these analytic expressions, the electric and hydrodynamic force can be determined by extracting the droplet radius from experiments. Drop sizes are extracted using edge detection in MATLAB. We chose the parallel gap for the force determination. In this case, droplets are always centered on the gap and show little deformation, which facilitates the calculation of the forces acting on trapped drops. Fig. 3-3 shows the drag force against the trapping force as experimentally determined for different flow velocities $u$ and droplet radii $R$. It shows that as long as the maximum electric trapping force exceeds the drag force a droplet will be trapped. Note that these electric forces are one to two orders of magnitude larger than the forces of 0.1 to $1 \mu N$ reported for geometric traps. ${ }^{15}$ Moreover, electrostatic traps offer the additional advantage of being switchable, enabling on-demand trapping and release of drops by changing the applied voltage.

\subsubsection{Applications}

\subsubsection{Droplet generation}

In our microfluidic chip droplets are generated by a flow focusing device (FFD). In a FFD water droplets are generated by the shear stress exerted by the oil phase (set at a certain flow rate) on the water interface. Changing the pressure balance between the oil and water phase changes generation frequency, inter-droplet distance and droplet size. Assuming the oil flow rate is set, droplet generation can be controlled in three ways.

Firstly, by using a syringe pump a continuous water flow can be provided, which results in a continuous generation of droplets. This mode is very stable and is thus particularly useful to create large amounts of droplets of uniform size at high frequency for longer periods of time.

Secondly, instead of using a syringe pump the pressure of the water channel can be governed by a pressure controller. At a certain pressure, the water interface will remain stationary at the tapered FFD-cross section. Providing a short pressure pulse to the water phase allows the generation of one droplet on demand. The size of the droplet is 
determined by the pulse duration $\Delta t$ and pressure difference $\Delta P$.

Thirdly, at the FFD our device is equipped with a single electrode below the water phase. The water phase itself is grounded, completing the electric circuit. Providing a voltage pulse to the electrode below the water phase actively pulls on the water interface, allowing the generation of droplets on demand. ${ }^{35,46}$ Similar to the pressure pulse, the size of the droplet is determined by the pulse duration $\Delta t$ and the voltage difference $\Delta U$.

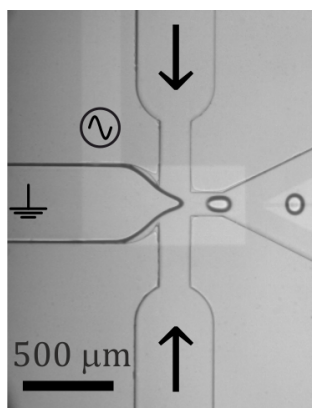

Fig. 3-4 In a flow focusing device water droplets are generated by the shear stress exerted by the oil phase (set at a certain flow rate) on the water interface. Droplet generation can be controlled in three ways: (1) a continuous water flow provided by a syringe pump; (2) a pressure pulse to the water phase gives on demand droplet generation; (3) a voltage pulse to the electrode below the water phase actively pulls on the water interface.

\subsubsection{Guiding of drops}

The principle that the electrostatic force will pull a drop towards the middle of a gap (as explained in Section 3.3.1.1) can be used to manipulate droplets. An example hereof is presented in Fig. 3-5. By placing the electrode gap at an angle $\alpha$ with respect to the flow direction, the electrostatic trapping force competes with the projection of the hydrodynamic driving force in the direction perpendicular to the gap. In terms of a simple force balance between the trapping and the driving force, this means that we can use the equations for a perpendicular gap, in which, however, the ratio between the hydrodynamic force and the maximum electrostatic force is now decreased by a factor of $\sin \alpha$. This allows for the guiding of drops even in strong flows, by designing a small value for $\alpha$. By combining several electrodes in a branching geometry, drops can be guided laterally along various predefined tracks, depending on which electrodes are activated. Only $n+1$ electrodes are needed to obtain $n$ possible directions. The path of subsequent drops can be easily altered by switching between the electrodes, which offers increased flexibility as compared to guiding along topological rails. ${ }^{14}$

\subsubsection{Parallel trapping of drops}

To further extend the trapping capability and practical usefulness of electric potential wells, we created six electrode arrays, each containing 6 trapping locations, as shown in Fig. 3-6. Firstly, drops are formed by the FFD, and are then guided along rails to 6 different parallel tracks. Further downstream droplets can be stopped using the independently operated arrays of electrostatic trapping wells located at the terminus of the guiding geometry. For each trapping array the gap between electrodes has a zigzagged shape that determines the trapping location for 6 droplets along the 6 parallel tracks. Fig. 3-6 shows the electrode array while trapping 36 droplets of different sizes. Switching off the potential releases all drops on an array which are subsequently dragged by the flow towards the next trapping array. 
This combination of guiding and trapping is very suitable in scenarios where several drops have to be chemically treated or analyzed in parallel for a certain amount of time; an example is the analysis of biomolecules in drops using local sensor surfaces (as e.g. in $\left.\mathrm{SPR}^{14}\right)$.
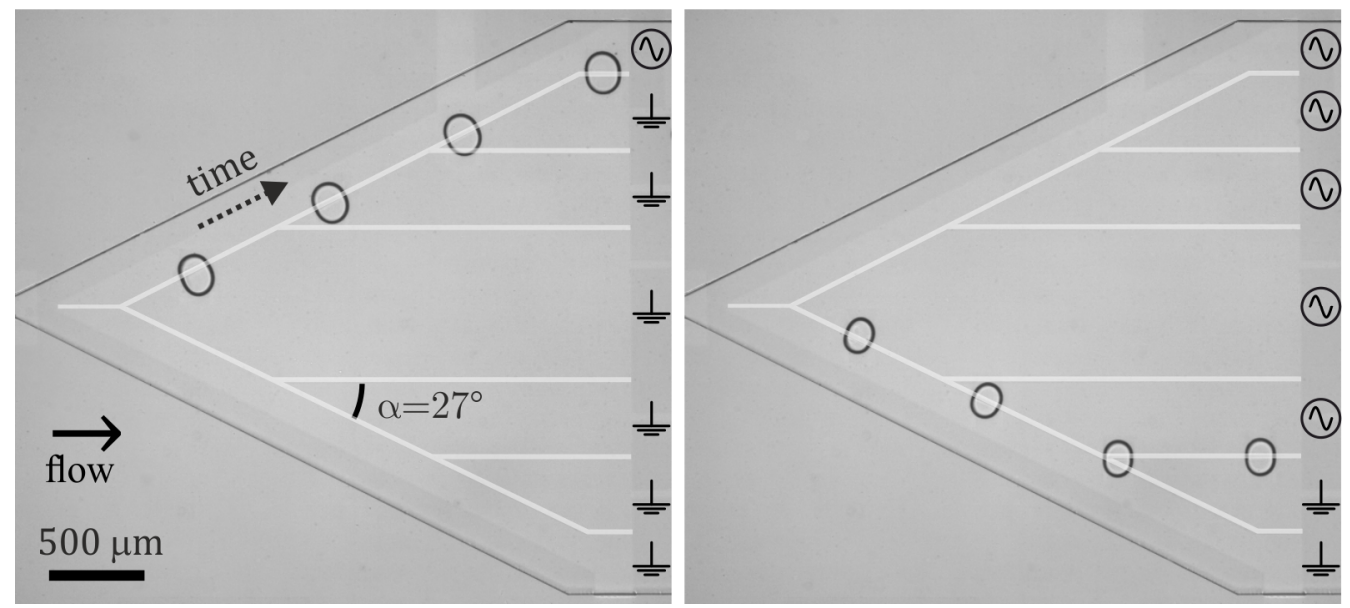

Fig. 3-5 Guiding of drops along various tracks in a microchannel using a series of individually addressable electrodes. These pictures are made by combining four time frames.

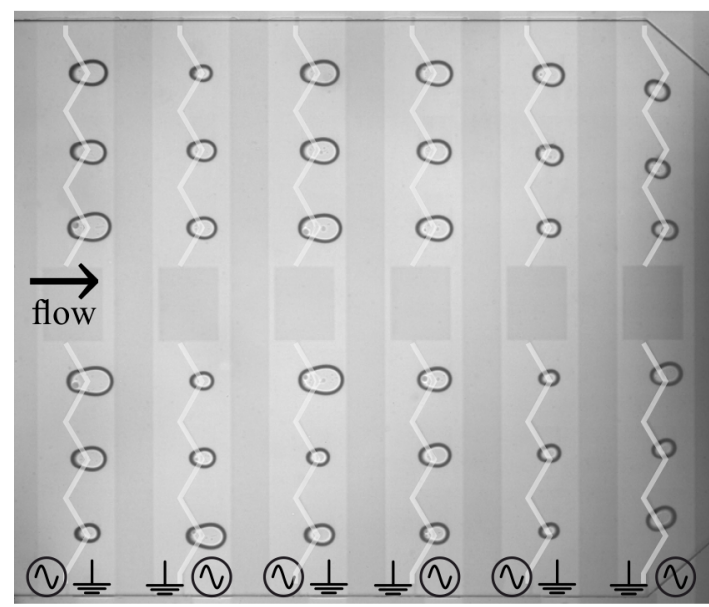

Fig. 3-6 Downstream of the droplet guider are trapping arrays for multiple droplets. The gaps between the electrodes are indicated in white. The trapping arrays can function as temporal storage and/or as a spot for diagnostics.

\subsubsection{Drop sorting}

The guiding of drops as shown in the Section 3.3.2.2 has an intrinsic speed limitation. As one track is activated, no other parallel track is accessible. Also, at higher flow speeds, drops are unable to follow the guide due to the drag force. To reduce the relevant component of the drag force, a smaller inclination angle is required, which results in longer 
tracks for reaching a certain lateral displacement. In lab-on-a-chip applications that involve sorting rather than slow analysis, for instance, selecting between empty and cellcontaining drops or between fluorescently labelled and non-labelled drops, higher handling speeds are desirable. This can be achieved by optimizing the electrode and channel design. To this end, we designed a three-electrode branching geometry underneath a Y-shaped channel (height $55 \mu \mathrm{m}$, width $400 \mu \mathrm{m}$ ) as shown in Fig. 3-7. An AC potential difference is applied continuously across the upper and lower electrodes, while the middle electrode is switched between the applied potential and ground electrode, effectively switching the path of the drop from one outlet to the other. Drops approach the Y-junction exactly in the middle of the channel and only require a small deflection to be directed to either outlet.
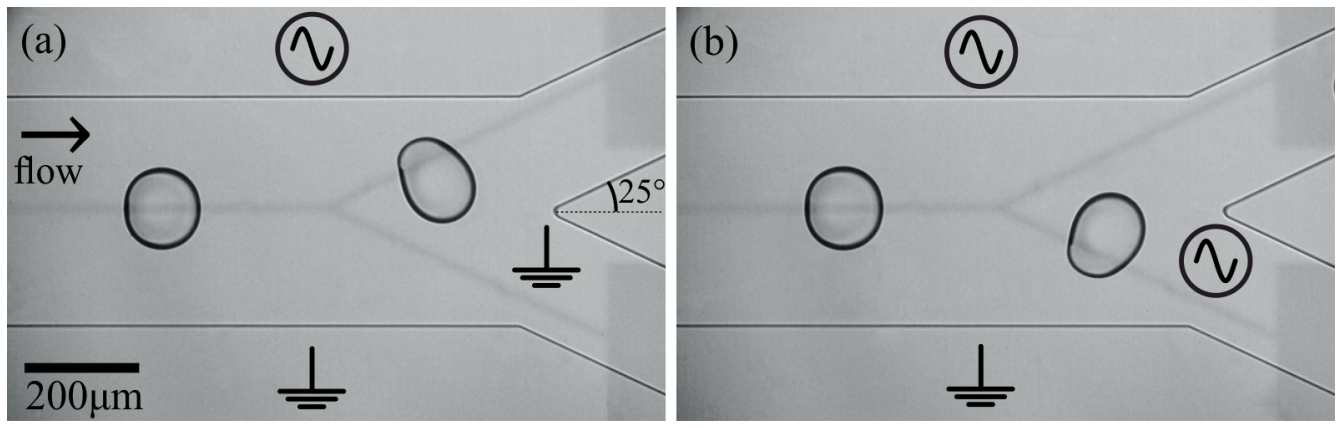

Fig. 3-7 Top view of a three-electrode sorting geometry below a Y-shaped channel. Drops are continuously generated using a flow focusing device. By switching the middle electrode (a) on and (b) off, a drop can be forced towards either outlet.

Similar to the previous experiments, we generate water drops in mineral oil $(\mu \approx 30$ mPas) using a flow focusing device. Depending on the flow rates of the water and oil phase, confined drops can be generated at 10 to $1000 \mathrm{~Hz}$. To avoid hydrodynamic interactions, the distance between subsequent drops can be increased by increasing the oil flow rate. The maximum electrostatic force is exerted when the center of the drop is at a distance of $\sim 0.67 R$ from the gap (see Section 3.3.1.1). The optimum design for deflecting drops that flow at a high speed (and hence experience large hydrodynamic forces) requires that the gaps are not centered below the outlet channels, but rather are offset towards the outer walls. With the gaps incorporated in this way, we observe that the drops end up approximately in the centers of the outlet channels. The applied potential is a $10 \mathrm{kHz}$ sine wave at $225 \mathrm{~V}$. For the middle electrode, the sine is modulated by a square waveform. When the middle electrode is grounded $(0 \mathrm{~V})$, drops are directed to the upper channel, and when it is at $255 \mathrm{~V}$, drops are directed to the lower channel. Modulating the middle electrode at half the frequency of drop generation allows alternate sorting of drops. It is also possible to select a single drop from a train of drops by applying a short pulse lasting one period of drop generation. In combination with a detection technique, e.g. optical or electrical, ${ }^{47}$ our device could be used as an active sorter. 
With the current device and materials used, we were able to sort 100 drops per second. At higher drop generation rates, the drag force becomes too large for the electrostatic force to pull the drop towards the desired outlet. However, the geometry of the channel and the electrodes could be optimized to achieve higher sorting rates. Since the drag scales linearly with the viscosity of the continuous phase, the drag could be reduced (and hence the sorting frequency is increased) by using oils with a lower viscosity. Another option is to decrease the inclination angle of the branching electrodes. In Chapter 5 these adaptations are implemented to increase the sorting frequency.

Electrostatic potential wells and dielectrophoresis (DEP) ${ }^{31,33}$ both make use of electric fields for drop sorting. DEP comprises the motion of liquid drops, particles, or biological cells in a non-uniform electric field and relies on differences in the (frequency-dependent) dielectric constant with the continuous phase. Obtaining the force for the actual device geometry thus requires calculation of the electric field gradients. In addition, sorting cellcontaining drops from empty drops in ambient oil involves the use of physiological strength buffers with a relatively large conductivity, i.e. typically $15 \mathrm{mS} \mathrm{cm}^{-1}$. Manipulation using DEP requires a frequency of hundreds of $\mathrm{MHz}$ in this case. ${ }^{48,}{ }^{49}$ In contrast, manipulation using electrostatic potential wells relies on the sharp contrast between conductive drops in an insulating medium. Our method is thus especially suitable for this application, while using lower frequencies and lower voltages as compared to DEP.

\subsubsection{Drop splitting}

Even though we ignored deformation in our force analysis, under the influence of electric actuation a droplet will of course change its shape via minimization of the sum of the electrostatic and interfacial energies. As the drop deforms perpendicular to the gap, the total area above the electrodes increases at the cost of the area above the gap. The resulting decrease in electric energy is balanced by the increase in interfacial energy associated with the increase in surface area. Thus, the presence of a gap in the co-planar geometry causes an indentation in the droplet shape. Minimizing the total energy then yields an equilibrium shape that is dependent on the applied voltage. This reasoning predicts larger drop deformations for wider gaps, higher voltages, and lower interfacial tension. For the purpose of limiting deformation in general we chose narrow gap widths and a high interfacial tension (i.e. no surfactants).

Extreme drop deformations result in the splitting of drops as shown for high voltages in Fig. 3-8. So while our simple force-based model would imply no limitations on the applied voltage and the absence of a role for the interfacial tension, it is the splitting of drops that limits the voltage that can be applied and thus the maximum electrostatic trapping force that can be exerted. In situations in which larger trapping forces are required or drops are sensitive to break-up (e.g. when surfactants are used that lower the interfacial tension), the use of electrodes in a parallel plate set-up could be considered. Here, the actuation electrodes are patterned on one substrate, while the ground electrode is on the other, which eliminates the presence of a gap. This approach can be used for both trapping and guiding of drops in channels. Although it is a restriction in the possibilities for drop trapping, the drop splitting itself offers another interesting drop manipulation in microchannels due to its symmetry. The original drop centers above the electrode gap due to energy minimization, and thus always splits into two equally-sized drops. 


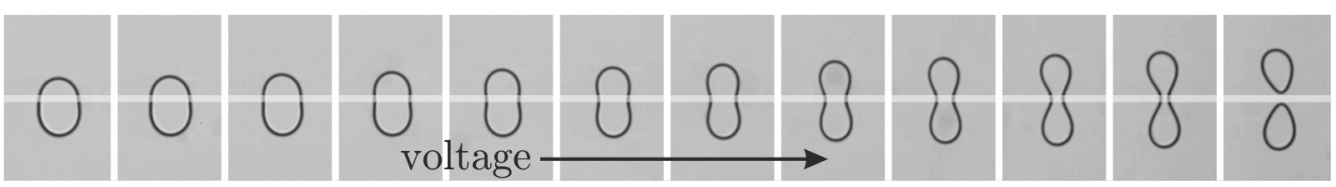

Fig. 3-8 Droplet deformation and splitting in oil flow at high voltage and large gap width. The gap between the electrodes is indicated in white.

\subsection{Conclusions}

We presented a hybrid concept for channel-based microfluidics with integrated electrowetting functionality to achieve individual control over pico- and nanoliter aqueous drops at high throughput. The trapping of drops using a co-planar electrode configuration is successfully predicted using a simple model that balances the maximum electrostatic force exerted by the trap with the hydrodynamic drag force exerted by the surrounding flow. Compared to droplet manipulation techniques using small geometric defects, the maximum forces are an order of magnitude larger and tunable. In addition, we showed trapping, guiding and sorting of drops. We anticipate that this hybrid technology platform will enable further flexible devices combining high throughput and individual drop control.

\section{References}

1. Song, H., D.L. Chen, and R.F. Ismagilov, Reactions in droplets in microflulidic channels. Angewandte Chemie-International Edition, 2006. 45(44): p. 7336-56.

2. Guo, M.T., A. Rotem, J.A. Heyman, et al., Droplet microfluidics for high-throughput biological assays. Lab on a Chip, 2012. 12(12): p. 2146-55.

3. Garstecki, P., M.J. Fuerstman, H.A. Stone, et al., Formation of droplets and bubbles in a microfluidic T-junction - scaling and mechanism of break-up. Lab on a Chip, 2006. 6(3): p. 437-46.

4. Thorsen, T., R.W. Roberts, F.H. Arnold, et al., Dynamic pattern formation in a vesiclegenerating microfluidic device. Physical Review Letters, 2001. 86(18): p. 4163-6.

5. Anna, S.L., N. Bontoux, and H.A. Stone, Formation of dispersions using "flow focusing" in microchannels. Applied Physics Letters, 2003. 82(3): p. 364-6.

6. Sugiura, S., M. Nakajima, S. Iwamoto, et al., Interfacial tension driven monodispersed droplet formation from microfabricated channel array. Langmuir, 2001. 17(18): p. 5562-6.

7. van Dijke, K., G. Veldhuis, K. Schroen, et al., Parallelized edge-based droplet generation (EDGE) devices. Lab on a Chip, 2009. 9(19): p. 2824-30.

8. van Dijke, K., R. de Ruiter, K. Schroën, et al., The mechanism of droplet formation in microfluidic EDGE systems. Soft Matter, 2010. 6(2): p. 321-30.

9. Baroud, C.N., F. Gallaire, and R. Dangla, Dynamics of microfluidic droplets. Lab on a Chip, 2010. 10(16): p. 2032-45.

10. Seemann, R., M. Brinkmann, T. Pfohl, et al., Droplet based microfluidics. Reports on Progress in Physics, 2012. 75(1). 
11. Gu, H., M.H.G. Duits, and F. Mugele, Droplets Formation and Merging in Two-Phase Flow Microfluidics. International Journal of Molecular Sciences, 2011. 12(4): p. 2572-97.

12. Boukellal, H., S. Selimovic, Y.W. Jia, et al., Simple, robust storage of drops and fluids in a microfluidic device. Lab on a Chip, 2009. 9(2): p. 331-8.

13. Sun, M., S.S. Bithi, and S.A. Vanapalli, Microfluidic static droplet arrays with tuneable gradients in material composition. Lab on a Chip, 2011. 11(23): p. 3949-52.

14. Abbyad, P., R. Dangla, A. Alexandrou, et al., Rails and anchors: guiding and trapping droplet microreactors in two dimensions. Lab on a Chip, 2011. 11(5): p. 813-21.

15. Dangla, R., S. Lee, and C.N. Baroud, Trapping Microfluidic Drops in Wells of Surface Energy. Physical Review Letters, 2011. 107(12).

16. Tan, Y.-C., Y.L. Ho, and A.P. Lee, Droplet coalescence by geometrically mediated flow in microfluidic channels. Microfluidics and Nanofluidics, 2007. 3(4): p. 495-9.

17. Niu, X., S. Gulati, J.B. Edel, et al., Pillar-induced droplet merging in microfluidic circuits. Lab on a Chip, 2008. 8(11): p. 1837-41.

18. Link, D.R., S.L. Anna, D.A. Weitz, et al., Geometrically mediated breakup of drops in microfluidic devices. Physical Review Letters, 2004. 92(5).

19. Salkin, L., A. Schmit, L. Courbin, et al., Passive breakups of isolated drops and onedimensional assemblies of drops in microfluidic geometries: experiments and models. Lab on a Chip, 2013.

20. Protiere, S., M.Z. Bazant, D.A. Weitz, et al., Droplet breakup in flow past an obstacle: A capillary instability due to permeability variations. Epl, 2010. 92(5).

21. Fradet, E., C. McDougall, P. Abbyad, et al., Combining rails and anchors with laser forcing for selective manipulation within 2D droplet arrays. Lab on a Chip, 2011. 11(24): p. 4228-34.

22. Wang, W., C. Yang, Y.S. Liu, et al., On-demand droplet release for droplet-based microfluidic system. Lab on a Chip, 2010. 10(5): p. 559-62.

23. Mugele, F. and J.C. Baret, Electrowetting: From basics to applications. Journal of PhysicsCondensed Matter, 2005. 17(28): p. R705-R74.

24. Fair, R.B., Digital microfluidics: is a true lab-on-a-chip possible? Microfluidics and Nanofluidics, 2007. 3(3): p. 245-81.

25. Pollack, M.G., R.B. Fair, and A.D. Shenderov, Electrowetting-based actuation of liquid droplets for microfluidic applications. Applied Physics Letters, 2000. 77(11): p. 1725-6.

26. Cho, S.K., H.J. Moon, and C.J. Kim, Creating, transporting, cutting, and merging liquid droplets by electrowetting-based actuation for digital microfluidic circuits. Journal of Microelectromechanical Systems, 2003. 12(1): p. 70-80.

27. Jebrail, M.J., M.S. Bartsch, and K.D. Patel, Digital microfluidics: a versatile tool for applications in chemistry, biology and medicine. Lab on a Chip, 2012. 12(14): p. 2452-63.

28. Zagnoni, M. and J.M. Cooper, On-chip electrocoalescence of microdroplets as a function of voltage, frequency and droplet size. Lab Chip, 2009. 9(18): p. 2652-8.

29. Priest, C., S. Herminghaus, and R. Seemann, Controlled electrocoalescence in microfluidics: Targeting a single lamella. Applied Physics Letters, 2006. 89(13).

30. Abdelgawad, M., M.W. Watson, and A.R. Wheeler, Hybrid microfluidics: a digital-to-channel interface for in-line sample processing and chemical separations. Lab Chip, 2009. 9(8): p. 1046-51.

31. Ahn, K., C. Kerbage, T.P. Hunt, et al., Dielectrophoretic manipulation of drops for high-speed 
microfluidic sorting devices. Applied Physics Letters, 2006. 88(2).

32. Wang, W., C. Yang, and C.M. Li, On-demand microfluidic droplet trapping and fusion for on-chip static droplet assays. Lab on a Chip, 2009. 9(11): p. 1504-6.

33. Baret, J.C., O.J. Miller, V. Taly, et al., Fluorescence-activated droplet sorting (FADS): efficient microfluidic cell sorting based on enzymatic activity. Lab on a Chip, 2009. 9(13): p. $1850-8$.

34. Malloggi, F., S.A. Vanapalli, H. Gu, et al., Electrowetting-controlled droplet generation in a microfluidic flow-focusing device. Journal of Physics-Condensed Matter, 2007. 19(46).

35. Gu, H., F. Malloggi, S.A. Vanapalli, et al., Electrowetting-enhanced microfluidic device for drop generation. Applied Physics Letters, 2008. 93(18).

36. Yi, U.C. and C.J. Kim, Characterization of electrowetting actuation on addressable single-side coplanar electrodes. Journal of Micromechanics and Microengineering, 2006. 16(10): p. 20539.

37. Malic, L., T. Veres, and M. Tabrizian, Nanostructured digital microfluidics for enhanced surface plasmon resonance imaging. Biosensors \& Bioelectronics, 2011. 26(5): p. 2053-9.

38. 't Mannetje, D., A. Banpurkar, H. Koppelman, et al., Electrically tunable wetting defects characterized by a simple capillary force sensor. Langmuir, 2013. 29(31): p. 9944-9.

39. Franke, T., A.R. Abate, D.A. Weitz, et al., Surface acoustic wave (SAW) directed droplet flow in microfluidics for PDMS devices. Lab on a Chip, 2009. 9(18): p. 2625-7.

40. Duffy, D.C., J.C. McDonald, O.J.A. Schueller, et al., Rapid prototyping of microfluidic systems in poly(dimethylsiloxane). Analytical Chemistry, 1998. 70(23): p. 4974-84.

41. Hele-Shaw, H.S., On the motion of a viscous fluid between two parallel plates. Trans. R. Inst. Nav. Archit., 1898. 40(218).

42. Jones, T.B., K.L. Wang, and D.J. Yao, Frequency-dependent electromechanics of aqueous liquids: Electrowetting and dielectrophoresis. Langmuir, 2004. 20(7): p. 2813-8.

43. Staicu, A. and F. Mugele, Electrowetting-induced oil film entrapment and instability. Phys Rev Lett, 2006. 97(16): p. 167801.

44. de Ruiter, R., A.M. Pit, V.M. de Oliveira, et al., Electrostatic potential wells for on-demand drop manipulation in microchannels. Lab Chip, 2014. 14(5): p. 883-91.

45. Vanapalli, S.A., A.G. Banpurkar, D. van den Ende, et al., Hydrodynamic resistance of single confined moving drops in rectangular microchannels. Lab on a Chip, 2009. 9(7): p. 982-90.

46. Gu, H., C.U. Murade, M.H. Duits, et al., A microfluidic platform for on-demand formation and merging of microdroplets using electric control. Biomicrofluidics, 2011. 5(1): p. 11101.

47. Kemna, E.W.M., L.I. Segerink, F. Wolbers, et al., Label-free, high-throughput, electrical detection of cells in droplets. The Analyst, 2013. 138(16): p. 4585-92.

48. Jones, T.B., J.D. Fowler, Y.S. Chang, et al., Frequency-based relationship of electrowetting and dielectrophoretic liquid microactuation. Langmuir, 2003. 19(18): p. 7646-51.

49. Pethig, R., Review article-dielectrophoresis: status of the theory, technology, and applications. Biomicrofluidics, 2010. 4(2). 


\section{4 \\ Hydrophilic patch}

In two-phase flow microfluidics there is an increasing interest in technologies which enable the encapsulation of biological cells into aqueous drops, and the subsequent study of their molecular (excretion or lysis) products. One not yet available but very promising analysis method is the use of biospecific surface patches embedded in the wall of microfluidic channels. In this paper we tackle some technological challenges encountered in the development of such applications. In the detection protocol, each drop must be enabled to wet the designated patch, be held in contact long enough for biomolecular detection, and subsequently be released. This is engineered via a combination of well-defined chemical sites in the walls of the flow channel, and insulated micro electrodes. The tunability of the local electric field allows to modify the competition between chemical pinning forces which tend to immobilize the drop, and hydrodynamic forces which oppose this process. We developed a prototype microfluidic device which offers this functionality. A channel structure is sandwiched between an actuation surface with electrowetting $(E W)$ electrodes on one side and a detector surface with a hydrophilic patch amidst a hydrophobic environment on the other. Two pairs of carefully aligned EW electrodes are used: one for drop adherence, and another one for the subsequent release. We demonstrate these operations, and discuss the required voltage signals in terms of the forces on the drop. Finally we discuss possible steps for further improvement of the device.

This chapter has been published as:

A.M. Pit, S. Bonestroo, D. Wijnperlé, M.H.G. Duits and F. Mugele, "Electrodeassisted trapping and release of droplets on hydrophilic patches in a hydrophobic microchannel", Microfluid Nanofluid 20(9): 123 (2016) 


\subsection{Introduction}

Microfluidics - in particular droplet based microfluidics - has become a very popular tool since its advent in the 1980s. Advantages include miniaturization, parallelization and compartmentalization, which - together with high speeds and the ability of upscaling result in cost reduction and enable the use of statistical analysis. ${ }^{1,2}$ Great examples of the use of microfluidics are large quantity emulsification (in food processing), inkjet printing and whole genome sequencing. ${ }^{3-5}$

One of the key application areas in microfluidics is biomedical diagnostics. ${ }^{6-8}$ In this field challenges arise in combining material properties with biochemical molecules, and in the detection of small quantities of analyte in complex samples. In particular the analysis of individual cells has become a topic of interest. ${ }^{9}$

The most common detection techniques are optical or electrical. ${ }^{10}, 11$ The dominant technique remains the fluorescence assay. However, the need to label with (large) fluorescent molecules can influence the chemical process under analysis. For this reason several non-labeling techniques for affinity-based pathogen sensing have been developed. ${ }^{12}$ One of these techniques is surface plasmon resonance imaging (SPRi). SPRi measures the change of refractive index which occurs when antigens from the water phase specifically bind to antibodies which are printed on gold. ${ }^{13,14}$

Continuous water flow SPRi has been proven capable of detecting and quantifying excreted molecules from single cancer cells over a period of 10 hours. ${ }^{15}$ For practical application it is preferable to do the analysis in a shorter time. The problem here is the dilution of the analyte in the fluid phase. This implies that, by using microfluidics to create small droplets $(<100 \mathrm{pL})$ SPRi could be capable of quickly measuring the excreted proteins of individual cancer cells.

While conventionally used for continuous flow measurements, SPR has previously been combined with droplet microfluidics. One thing must be noted: by using protocols involving individual droplets that have to perform complicated tasks, a manipulation method is required. Many methods have been developed. ${ }^{16-18}$ For combination with SPR pneumatic membrane valves ${ }^{19}$ and electro-wetting-on-dielectric (EWOD) ${ }^{20,21}$ have been used.

A key obstacle for the further development of these promising droplet-based SPR experiments is, that once the aqueous droplet interacts with the (hydrophilic) biomolecules on the substrate, the pinning force may be so strong that the droplet cannot be removed again. This issue has so far been mitigated by using relatively large droplets compared to the hydrophilic patch size. When using 100pL droplets, however, and keeping in mind that the technique of SPRi requires gold patches to be at least in the order of tens of microns, ${ }^{22}$ the size of patch and droplet are practically equal. This underlines that important cases exist, in which the problem of the strong pinning force cannot be circumvented by using larger droplets; another strategy is needed.

We are particularly interested in the combination of droplet based microfluidics with SPRi for diagnosis of various excreted products of an individual cancer cell, while the droplet is still in flow. Key challenges are: 1) droplets have to be held in place at a detections site long enough for analysis, 2) they have to be released and transported to the next measurement site. For the trapping of droplets passive ${ }^{23,24}$ and active ${ }^{25,26}$ methods 

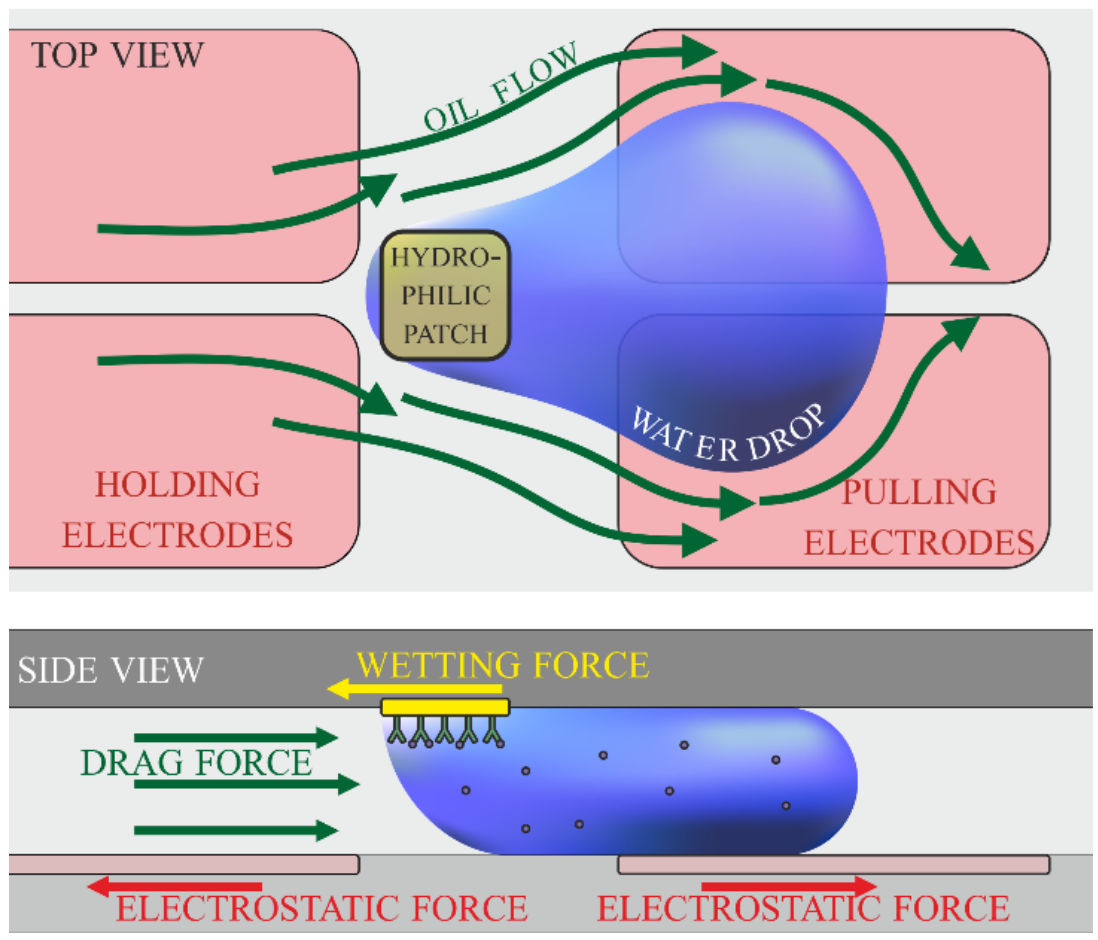

Fig. 4-1 Schematic overview of the desired functionality of a microchip where a droplet interacts with an antibody covered gold patch while in oil flow and with electrodes for holding a drop, and pulling it off.

have been developed. For active release the options are mainly restricted to using higher flowrates, of which the pressure changes can be impractical, e.g. for droplet generation, in microfluidic chips.

In this paper we explore a new method for controlling both the adherence and the detachment of droplets on designated hydrophilic patches inside a microfluidic chip. Here, the droplets are in the pL size regime, and ambient oil is used to isolate and transport the droplets. These design aspects are very well suited for applications with individual cells in drops; not only are the dimensions in the proper range, but also the use of an oil flow meets the practical requirements very well: droplets can be generated and sorted at high rates, ${ }^{27-29}$ and both losses of water and biomolecules to the environment are minimized. This high speed droplet generation and screening are for instance required for isolating circulating tumor cells, of which a patient has very few.

The key novelty is the design of a multifunctional chip that contains hydrophilic patches amidst a hydrophobic environment, and two sets of insulated electro-wetting electrodes for drop manipulation. Multiple challenges were overcome with regards to fabrication, material properties, alignment and sealing. Several micro and macro fabrication techniques are combined and developed, which are explained in detail.

Besides the design and fabrication of the chip, the theory of the relevant forces is described. The forces are calculated and compared to experiments involving the trapping 
of droplets, the pinning of a droplet to the hydrophilic patch and the subsequent release from the patch by the secondary electrode pair.

\subsection{Device concept}

Our final, envisioned device will incorporate a drop generator, cell sorter ${ }^{29}$ and various storage and detection arrays, as shown in de Ruiter, et al. $(2014)^{26}$ Here, we focus on overcoming the difficulties that result from working with wetting defects. The principal features of our method are illustrated in Fig. 4-1. Each pair of EW electrodes separated by a narrow gap serves as an energy well for the aqueous drop, where the depth of the well (or equivalently the attractive force) can be controlled via the voltage difference between the two. When properly designed, droplets can be manipulated by the electrodes without the need to change the oil flow rate.

Two key issues have to be addressed:

\section{The thin oil film between droplet and channel wall}

A stationary droplet in oil will wet a substrate to obtain an equilibrium contact angle. However, when a droplet flows in oil (in an oil-wetting channel), a thin oil film is present between the droplet and the wall. This means that a droplet flowing over a hydrophilic patch will be lubricated by a thick oil film. It will most likely not feel the presence of that patch and pass without being trapped. We therefore use a pair of electrowetting electrodes to trap the droplet above the patch until the oil film breaks up.

The competition between electric -, pinning -, and drag force

Before the oil film can break up, the droplet first has to be trapped above the patch. Therefore, the electric (trapping) force will have to be stronger than the viscous drag force due to the flowing oil. Once the droplet has spread over the hydrophilic patch the pinning force has to be stronger than the drag force. In this way the droplet can be passively held in place. Finally, to remove the droplet from the patch the combination of electric pulling (by activating the electrodes downstream of the patch) and the drag force together need to exceed the pinning force to release the drop (See Fig. 4-1).

For creating a microfluidic prototype we focus on solving the aforementioned issues. We have previously shown that electrodes are capable of trapping droplets in oil flow, ${ }^{26}$ and thus it makes sense to use electrodes as the active force for holding a droplet in flow, until it wets the hydrophilic patch. Likewise, a second pair of pulling electrodes (together with the oil drag) should be capable of pulling the pinned droplet off the patch.

\subsection{Material \& Methods}

\subsubsection{Motivation of design choices}

A thin oil film between electrode and droplet can actively be broken up using electrowetting. ${ }^{30}$ However, SPR requires a flat substrate with few refractive index changes. It is preferred to apply gold (and chromium) directly to glass, without a layer of electrodes, 
insulator, or hydrophobicity in between or on top. For this reason it was decided to make a two-sided microchip, and to make the two sides modular. The actuation chip half contains the electrode geometries aligned to a thin slab of PDMS, which forms the (open) microchannel and simultaneously functions as the insulating layer required for electrowetting. The sensing chip half consists of a hydrophobized glass slide with inlets, and small hydrophilic gold patches, which resemble printed antibodies as used in SPRi.

This modularity increases the universality of our microfluidic device, where the two chip halves are individually replaceable. Modularity also makes integration of the electrode and open channel chip with other existing diagnostic tools easier. Changing from a onesided to a two-sided device does however require a completely new fabrication protocol. Instead of attaching a PDMS channel to electrodes already insulated by $3 \mu \mathrm{m}$ of PDMS, ${ }^{29}$ we now create a thin $(\sim 55 \mu \mathrm{m})$ slab of PDMS functioning as microchannel and insulator in one. This slab is directly spun, aligned, and cured atop the electrode structure, as explained in the following sections. Eventually a fabrication protocol was developed that incorporates all the chip requirements and results in nearly perfect chip alignment without failure.

\subsubsection{Materials}

An overview of the microfluidic device is given in Fig. 4-4. Upstream, a flow focusing device (FFD) allows for continuous or on-demand generation of droplets. The water phase is phosphate buffered saline (PBS, $\sigma=15 \mathrm{mS} / \mathrm{cm}$ ). The continuous phase, controlled by a syringe pump, is light mineral oil $(\mu=31.5 \mathrm{mPa} s)$, which wets both the PDMS and Cytop well. By applying a controlled pressure pulse (voltage, duration) to the water phase it is possible to create droplets on-demand with a radius ranging from 15 to $40 \mu \mathrm{m}$. Applying a continuous higher pressure allows to generate uniform droplets continuously at $0-300 \mathrm{~Hz}$ rates.

The electrode geometry consists of only three large electrodes of which one is grounded, and the other two can be grounded or active (see Fig. 4-4d and Appendix 4A for the masks). These three electrodes form five consecutive areas where a droplet can be trapped and released. Each structure has two functions. The first electrode pair is designed for temporarily trapping the droplet. The second electrode pair is designed for release of the droplet from the hydrophilic patch.

The gold patches are located with the same pitch as between the electrode structures, and at the same distance from the inlets. Since the powder blasted inlets are $\sim 500 \mu \mathrm{m}$ in diameter, this allows plenty of error for the final alignment of the gold patches to the electrode geometry, which does have to be precise.

\subsubsection{Electrowetting actuation surface (fabrication)}

The actuation chip half consists of a glass substrate with ITO patterned electrodes, a PDMS insulating layer and microchannel. The fabrication process is schematically shown in Fig. 4-2. Firstly the mold for the channel is created. A $52 \mu \mathrm{m}$ layer of SU8 is spun over a silica wafer. Using standard photolithography the shape of the inversed channel surrounded by support pillars are soft baked, exposed to UV and crosslinked. Before dissolving the uncured SU8 a secondary layer of $3 \mu \mathrm{m}$ SU8 is spun over the first layer. 
(a)
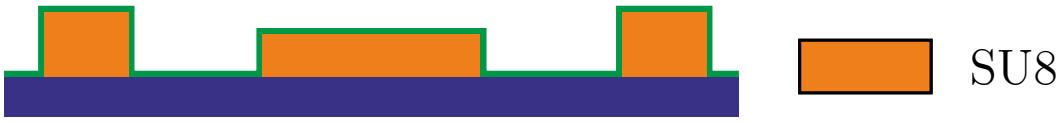

(b)
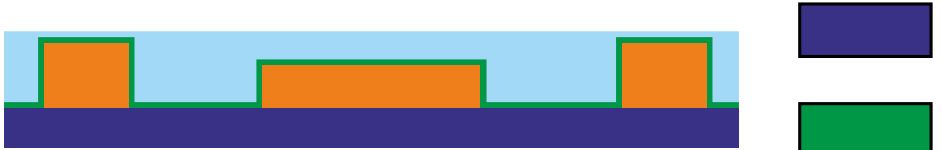

Silica

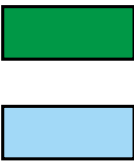

FOTS

(c)

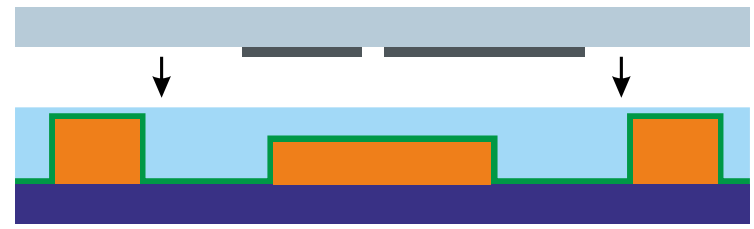

PDMS

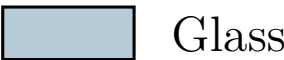

ITO

(d)

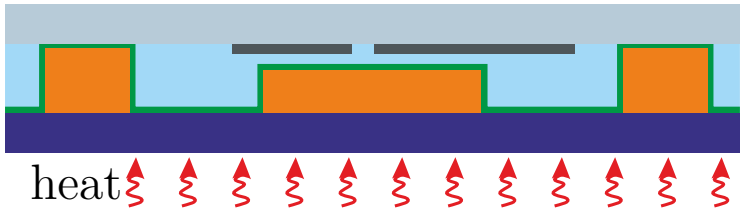

(e)

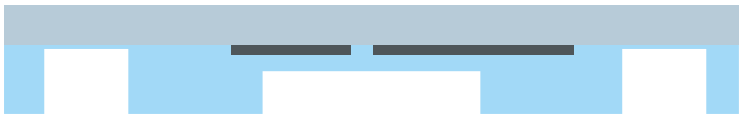

Fig. 4-2 Fabrication steps of first chip half. (a) Start with hydrophobized SU8 channel mold atop silica. (b) Spin slight excess of PDMS over channel and pillars. (c) Align ITO electrodes with the channel and press together. (d) Use Peltier element to cure PDMS at $70^{\circ}$ for 2 hours. (e) Remove the mold to be left with ITO electrodes, covered by a $\sim 3 \mu \mathrm{m}$ dielectric layer and perfectly aligned in the center of the PDMS channel.

For this second layer only the pillar structures next to the channel are exposed and crosslinked. After developing the uncured SU8 a mold is left with a $52 \mu \mathrm{m}$ high channel structure surrounded by support pillars that are $3 \mu \mathrm{m}$ higher. The mold is then coated with a monolayer of FOTS by vapor deposition under vacuum. This ensures hydrophobicity and facilitates easy removal of the cured PDMS layer from the following process steps.

Next, a $58 \mu m$ layer of PDMS with 10:1 PDMS:curing agent (Sylgard 186) ratio is spun over the mold ensuring a slight excess of PDMS covering the channel and pillars. Using a homebuilt stereomicroscope, which includes 2 translational stages $(x, y, z), 1$ rotational stage, and vacuum suction to hold both the upper and lower substrate in place, the PDMS covered mold is placed atop a Peltier element on the microscope and is subsequently aligned to a substrate with patterned ITO electrodes. The ITO pattern contains - besides the electrowetting electrodes - alignment markers which follow the exact shape of the channel to aid in the alignment process (See Appendix 4A and Fig. 4-4d).

The Peltier element, mold and electrodes are pressed together on the stereomicroscope, taking care not to encapsulate air bubbles in the corners of the microchannel. Since the 
PDMS is still liquid, an accidental imperfect alignment can still be corrected by simply moving the translational and rotational stages of the alignment microscope. Lodged on the microscope, the Peltier is now set to $70{ }^{\circ} \mathrm{C}$ for 2 hours to ensure the PDMS is fully cured. Since the PDMS bonds covalently to the glass and ITO electrodes, whereas the mold is coated with FOTS, the two substrates are effortlessly separated after curing, allowing the mold to be reused. Because the support pillars of the mold around the channel are $3 \mu \mathrm{m}$ higher than the channel, the electrodes are covered by a $3 \mu \mathrm{m}$ layer of PDMS, which is needed as insulation for electrowetting. The final actuation chip half consists of a glass substrate with electrode pattern, insulating layer and aligned channel.

\subsubsection{Functional patch surface (fabrication)}

The sensing chip half consists of a hydrophobized glass substrate with hydrophilized gold patches and comprises access holes for the liquids. The fabrication process is schematically shown in Fig. 4-3. Firstly tapered access holes for the water and oil inlets and the outlet are powder blasted in a glass wafer. The access holes are temporarily closed off by scotch tape, and $1 \mu \mathrm{m}$ of Cytop is spun on the opposite side. Putting the wafer in the oven at $80{ }^{\circ} \mathrm{C}$ for 30 minutes ensures the solvent is evaporated. Then the oven is set to $180^{\circ}$ for 90 minutes, which ensures the glass transition temperature of Cytop is reached, creating a solid hydrophobic layer of homogeneous thickness. The oven is allowed to slowly cool down to room temperature to prevent cracks forming in the Cytop layer. A shadow mask with square holes, created by $\mathrm{KOH}$ etching of a silicon wafer, is aligned with the access holes. The glass wafer and shadow mask are placed in a reactive ion etching (RIE) machine where the Cytop is etched by $\mathrm{O}_{2}$ plasma at the locations where eventually the hydrophilic patches will be in the microchannel. Then chromium $(5 \mathrm{~nm})$ and gold $(100 \mathrm{~nm})$ are sputter deposited atop the mask. The mask is removed leaving behind $\sim 45$ by $45 \mu \mathrm{m}$ square patches of gold on glass surrounded by Cytop. The substrate is diced in individual

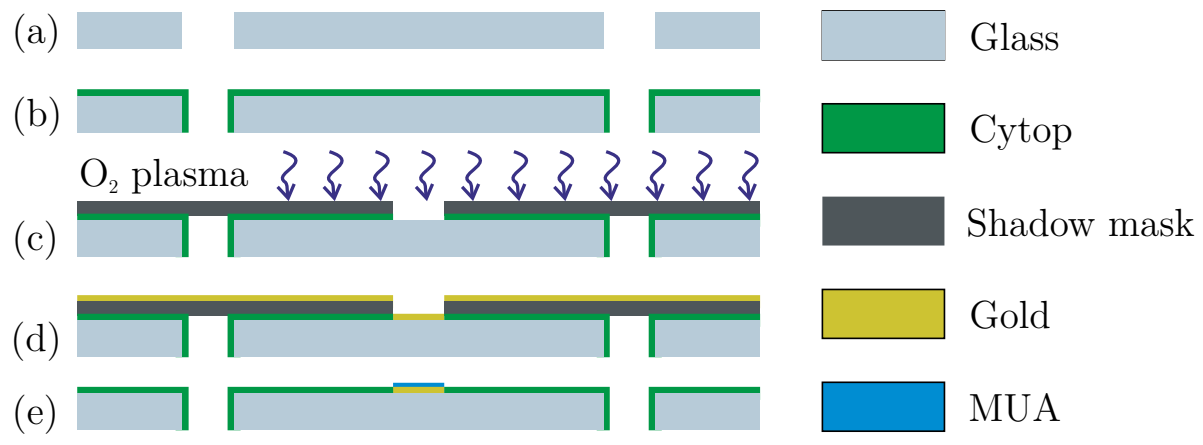

Fig. 4-3 Fabrication steps of second chip half. (a) Start with glass slide with powderblasted inlets. (b) Spin and cure $1 \mu \mathrm{m}$ of Cytop. (c) Use shadow mask to burn away patches of Cytop with $\mathrm{O}_{2}$ plasma. (d) Sputter gold. (e) Remove mask and place substrate in 2\% 11-mercaptoundecanol (MUA) to hydrophilize the gold patches. Rinse with ethanol to remove excess MUA and be left with a hydrophobic substrate with hydrophilic gold patches. 
chips. These chips are put in a $1 \mathrm{mM}$ solution of 11-mercapto-undecanol (MUA) in ethanol for 30 minutes, which creates a covalently bound hydrophilic monolayer on the gold patches. The chips are flushed with ethanol and dried with nitrogen. The final sensing chip half consists of a glass substrate with microfluidic access holes, a completely hydrophobic Cytop coating, except for the gold patches which are coated with a hydrophilic monolayer of MUA.

\subsubsection{Chip assembly}

The stereomicroscope is used to align the two chip halves so the hydrophilic gold patches are centered between the trapping and release electrodes. While the connection between PDMS and Cytop provides enough friction to prevent lateral movement after alignment, there is no chemical bonding between the PDMS channel and the Cytop. As an advantage, the chip halves can still be separated and realigned if alignment is not satisfactory. On the other hand, to prevent leaking of the microchannel neodymium magnets are used on all sides of the microchannel that press the chips together.

For connecting tubing to the inlets a PDMS block with punched holes is used. The PDMS block and microchip are treated by oxygen plasma for $30 s$ and then immediately brought together to form a permanent seal. The tubing can be directly pressed into the access holes for a leak free connection. Finally electric wiring is glued to the electrode patches using silver glue, as seen in Fig. 4-4c.

(a)

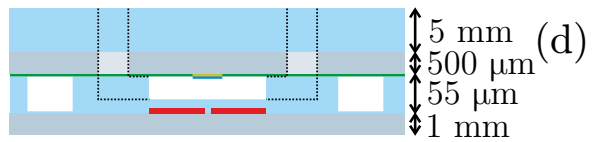

(b)

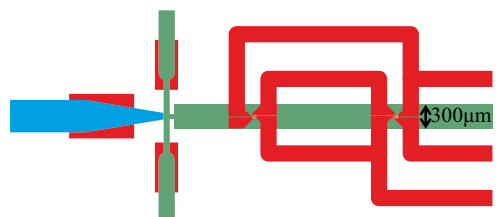

(c)

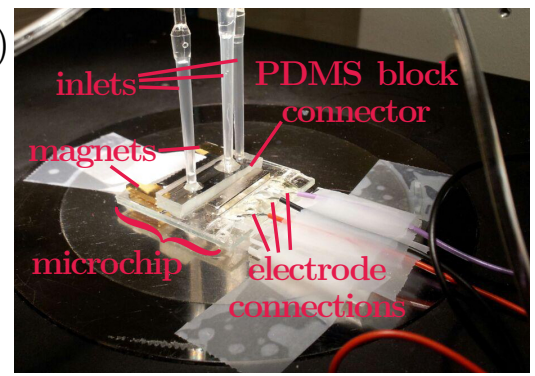

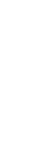

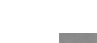




\subsection{Theory}

In our microfluidic device there are three important forces acting on the droplets: the electrostatic, drag and pinning force. Estimations of these forces have been presented previously $^{26,31,32}$ and we give only a short synopsis here.

\subsubsection{Electrostatic forces}

The electrostatic force, also named electrowetting or dielectrophoretic force, originates from the difference in capacitive energy when the material between two electrodes is displaced by another material with higher permittivity (a larger dielectric constant). In our microfluidic channel it is a conductive acqueous droplet that displaces the oil, while it spreads over two co-planar electrodes. For simplicity we ignore the electrostatic energy when oil is between the electrodes. Then, the electrostatic energy that is gained when a conductive droplet displaces the oil is given by the capacitance of, and voltage applied over, the dielectric layer of PDMS atop the electrodes: ${ }^{33}$

$$
W_{e}=-\frac{1}{2} C U^{2}=-\frac{1}{2} \frac{\epsilon_{0} \epsilon_{r}}{d} \frac{A_{1} A_{2}}{A_{1}+A_{\imath}} U^{2}
$$

with $\epsilon_{0} \epsilon_{r}$ the permittivity of PDMS, $d$ the thickness of the PDMS layer, $A_{1}$ and $A_{2}$ the position dependent areas the droplet covers over the grounded and active electrode, and $U$ the applied potential over the electrodes. The electrostatic force is the derivative of the change in energy with respect to position, with a maximum given as:

$$
F_{e, \max }=-\frac{d E_{e l}}{d x} \approx a \frac{\epsilon_{0} \epsilon_{r}}{d} R U^{2}
$$

with $a$ a geometrical factor depending on electrode geometry and $R$ the droplet radius. The direction of the electrostatic force is as follows: when a droplet covers two electrodes of different potential, the force is directed towards the gap between the electrodes. When a droplet is pushed of the electrodes by for instance drag, the electrostatic force will oppose this motion.

\subsubsection{Hydrodynamic drag force}

The hydrodynamic drag force $F_{d}$ on a stationary drop can be estimated by modelling the drop as a (cylindrical) pillar with no-slip boundary conditions in an infinitely wide channel. Since the drop diameter is about one sixth of the channel width we chose to neglect the effect of the channel walls on the drag force. This yields: ${ }^{26}$

$$
F_{d}=\left(\frac{24 \pi \mu u_{o i l} R^{2}}{h}\right)\left(1+\frac{2 K_{1}(q)}{q K_{0}(q)}\right)
$$

with $\mu$ the viscosity of the oil, $u_{\text {oil }}$ its velocity, $R$ the pillar (i.e. drop) radius, $h$ the channel height, and $K_{1}$ and $K_{2}$ modified Bessel functions of the second kind with $q=$ $2 \sqrt{3} R / h$. We also assume a constant oil velocity in the center region of the channel. 


\subsubsection{Pinning force}

The pinning force originates from a difference in wettability where a droplet interacts with the substrate. A droplet will prefer to be present on a hydrophilic patch and thus a force will be required to remove the droplet from this patch. This force can be estimated as: ${ }^{31}$

$$
F_{w e t}=-\gamma_{w o} \cdot\left(\cos \theta_{\text {phobic }}-\cos \theta_{\text {philic }}\right) \cdot \frac{d A_{p}(x)}{d x}
$$

with $\gamma_{w o}$ the water oil interfacial tension $(\sim 50 \mathrm{mN} / \mathrm{m}), \theta_{\text {phobic }}$ and $\theta_{\text {philic }}$ the Young angles on the hydrophobic Cytop, and the hydrophilic patch, respectively, and $A_{p}$ the position dependent area of the droplet overlapping the patch.

Assuming the droplet completely covers the entire width of the patch at some point, the maximal pinning force becomes:

$$
F_{\text {wet }, \text { max }}=\gamma_{w o} \cdot\left(\cos \theta_{\text {philic }}-\cos \theta_{\text {phobic }}\right) \cdot w
$$

with $w$ the width of the patch.

\subsection{Results}

\subsubsection{Droplet trapping}

In the first set of experiments, we use a microfluidic device containing the actuation surface but not the patch surface to study the electrostatic trapping of droplets, against the drag force. Maintaining the oil flow rate at $400 \mu L / h$, and setting the water pressure to continuously generate droplets of $R \sim 30 \pm 4 \mu \mathrm{m}$. The $\mathrm{EW}$ voltage $U$ was increased from 50 to $250 V_{R M S}$ while the $\mathrm{AC}$ frequency was kept at $4 \mathrm{kHz}$. At increasing voltages droplets are trapped and will coalesce with consecutive droplets (see also Fig. 4-6a and Movie 4-1) until the hydrodynamic drag force exceeds the trapping force and the droplet is pulled off. The radius $R^{*}$ of these droplets was measured downstream of the trapping site and is plotted against $U^{2}$ in Fig. 4-5.

The graph shows that below $130 \mathrm{~V}$ the droplets do not get trapped, although a reduction in the droplet velocity while passing the electrodes is observed. At $140 \mathrm{~V}$, primary droplets are trapped if the electrodes are not yet occupied. When a secondary droplet arrives, an instantaneous coalescence occurs, after which the merged droplet is released. This can be understood from a shift in the force balance: the drag force increases more strongly with increasing drop radius than the electric trapping force. Further raising the voltage from 140 to $190 \mathrm{~V}$ we see a linear increase of $R^{*}$ with the (mean) squared voltage. This is in good accordance with the theory, which predicts that the drag force scales as $R^{2}$, while the maximal electrostatic force scales as $R U^{2}$. Since a droplet should be released when $F_{d}>F_{e, \max }$ the slope in the green region $R^{*} / U^{2}$ in Fig. 4-5 should roughly be constant.

To analyze the force balance in more detail, we calculated the electrostatic and viscous forces for those (coalesced) droplets that are on the brink of being dragged off the 


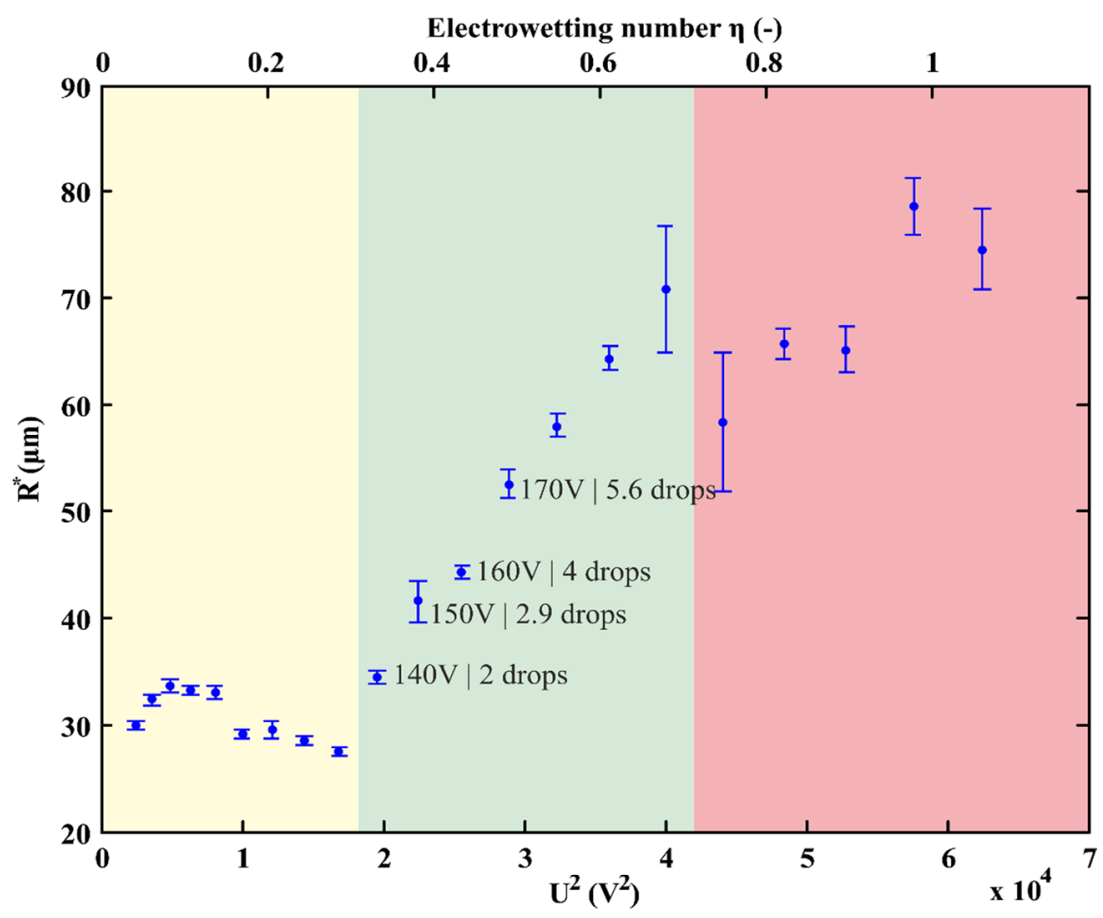

Fig. 4-5 Radius of the droplets after interaction with the (the first pair of) trapping electrodes at potential $U$. The yellow region corresponds to primary droplets passing the electrodes. In the green region drops are trapped, and consecutive drops coalesce with the trapped droplet until the drag force overcomes the electrostatic force. Also the actual applied voltage and the average amount of droplets trapped are given for the first four measurements in this region. In the red region (corresponding to $E W$ numbers $\geq 2.9$ ) saturation occurs, a common observation in electrowetting.

electrodes. The drag force was obtained from Eq. 4.3, by inserting the half width of the (slightly deformed) drop for the radius $R$. The electrostatic force was calculated numerically, since the analytic Eq. 4.2 (right hand side) considers neither the gap between the electrodes, nor the electrode shape explicitly. Using image analysis, we first extracted the shape of the droplet, the electrode geometry and the areas $A_{1}$ and $A_{2}$ of the trapped drop on the active and ground electrodes. This allowed to calculate the electrostatic energy from Eq. 4.1. Then we determined the local energy landscape, by artificially moving the droplet shape over the electrode geometry (a few pixels back and forth in the flow direction). This enabled us to obtain the electrostatic force (See Appendix 4B) by taking the numerical derivative as in Eq. 4.2.

The results are presented in Table 4-1. Even though we should expect the forces to be nearly equal (the drag force should only be slightly favored), it turns out that (for all four cases) the calculated electrostatic force is a factor 3 larger than the calculated drag force. Given the approximations in the theory and slight errors in input parameters, this 
systematic deviation is to be expected.

Besides the linearity between $R^{*}$ and $U^{2}$, the green region of Fig. 4-5 also shows the ability to coalesce 2, 3, 4 or 5 droplets at the trapping electrodes. Compared to other methods for electric field induced merging of droplets, ${ }^{34,}{ }^{35}$ our technique has two advantages: the number of coalescing droplets is flexible and can be controlled via the voltage, and the droplets do not need to reach the electrodes simultaneously: the first droplet is simply trapped and waits for the consecutive droplet(s) to merge, after which the final drop is released automatically (See Appendix 4D and Movie 4-1).

For voltages above $190 \mathrm{~V}$, a saturation effect is observed: raising the voltage further does not seem to increase the electrostatic (trapping) force. This is a common occurrence in EW, where the three phase contact line becomes unstable as the electric field strength increases near the sharp contact angle. ${ }^{36}$ Charges - attracted to the interface by the electric field - start repelling each other so strongly that small (charged) satellite droplets are ejected from the mother droplet. This behavior was also observed in our experiments, at voltages above $190 \mathrm{~V}$, and specifically when the droplet was dragged off the electrode (the electric field is strongest at that point). Electrowetting phenomena are generally mapped using the EW number $\eta=\epsilon_{0} \epsilon_{r} U^{2} / 2 d \gamma,{ }^{36}$ with $\gamma$ the oil-water interfacial tension $(50 \mathrm{mN} / \mathrm{m})$. Since our geometry uses two co-planar electrodes it is common to use a correction factor of $\frac{1}{4}$ in the definition of $\eta$ to account for the reduced voltage at each electrode contact $\eta=\epsilon_{0} \epsilon_{r} U^{2} / 8 d \gamma$. This quantity is plotted as the upper axis in Fig. 4-5. The regime where our experimental data level off, corresponds to $\eta \geq 0.7$. Note, however, that the actual 'effective' voltage is higher if the overlap area between the drop and two electrodes is not symmetric. ${ }^{32}$

Another observation at these high voltages, is that wetting defects (locations where droplets tend to adhere without control) are formed in the direct vicinity of the electrodes. Since we did not observe any signs of dielectric breakdown (like gas bubble formation), we consider it most likely that these defects occur because water is pulled into the porous PDMS layer, displacing oil which previously filled the PDMS.

\subsubsection{Droplet wetting the patch}

In the following experiments the two-sided chip as described in Section 4.3 was used, containing electrode structures for EW trapping and release of droplets on one side, and

Table 4-1 Calculated maximal electrostatic and drag forces on trapped droplets at increasing voltages $U$ and the corresponding radii $R$

\begin{tabular}{|c|c|c|c|}
\hline $\boldsymbol{U}(\boldsymbol{V})$ & $\boldsymbol{R}(\boldsymbol{\mu m})$ & $\boldsymbol{F}_{\boldsymbol{e}}(\boldsymbol{\mu} \boldsymbol{N})$ & $\boldsymbol{F}_{\text {drag }}(\boldsymbol{\mu N})$ \\
\hline 140 & 34 & 0.85 & 0.26 \\
150 & 42 & 1.4 & 0.37 \\
160 & 44 & 1.6 & 0.42 \\
170 & 52 & 2.2 & 0.59 \\
180 & 58 & 2.3 & 0.73 \\
\hline 190 & 64 & 2.9 & 0.90 \\
\hline
\end{tabular}


hydrophilized gold patches on the other. Droplets of Phosphate Buffered Saline were generated on demand using pressure pulses. After setting the flow rate of the mineral oil to $285 \mu \mathrm{L} / \mathrm{h}$, the potential of the trapping electrodes was adjusted to $300 V_{R M S}$ to ensure a stable trapping. The requirement of a higher voltage (compared to the trapping experiments in Fig. 4-5) indicates that the dielectric layer was thicker this time (see our earlier remarks). The AC frequency of the applied voltage signal was $4 \mathrm{kHz}$.

In the absence of a trapping voltage, the droplets flow past the patch without showing any visible change in shape or velocity (see Movie 4-2). Apparently the aqueous droplets do not have enough time to 'sense' the hydrophilic patch, and squeeze out the hydrodynamically entrapped oil. This confirms that the trapping electrodes are needed to force the drop into direct contact with the sensing patch. The electrostatic trapping of droplets proceeds in a similar way as with the other chip: as long as the maximum electrostatic force $(\propto R)$ remains larger than the drag force $\left(\propto R^{2}\right)$, the generated droplets keep collecting and merging at the trap (see Fig. 4-6a). Due to the use of pressure pulses, the primary droplets now had a radius $R \sim 16 \mu \mathrm{m}$. Up to 7 primary droplets could be collected in this way.

It turned out that for droplets with $R \sim 32 \mu \mathrm{m}$, a trapping time of a few seconds was sufficient to achieve wetting of the patch. This was not directly visible on the microscope, but became apparent after grounding both trapping electrodes: the droplet was not picked up by the flow, but did undergo a small downstream displacement. In this process the droplet slowly increased its coverage of the patch until it was complete. The droplet remained stuck to the patch, indicating that the pinning force was larger than the drag force (see Fig. 4-6b and Movie 4-3).

\subsubsection{Droplet pull-off}

To detach the drop from wetted the patch, we employed the second set of EW electrodes (situated on the right hand side of the patch in Fig. 4-6a). As in the previous experiments, the oil flow rate was kept constant at $285 \mu L / h$. Initially we applied a similar AC voltage signal as for trapping $(300 \mathrm{~V}, 4 \mathrm{kHz})$. Here we observed that, although the droplet did get deformed as the EW force pulled on the droplet, it remained stuck on the patch (not shown). This indicates that the pinning force is so strong that it can even resist the sum of the electrostatic and drag forces.

In most EW applications, the AC frequency is chosen to be high $(>1 \mathrm{kHz})$. This serves to 'shake' the contact line of the drop and hence facilitate depinning, ${ }^{37}$ without causing a vigorous center-of-mass motion. Since the droplets could not get released at $4 \mathrm{kHz}$, we explored a much lower frequency, expecting that this would result in a mechanical excitation of the entire droplet which was also sufficiently prolonged to allow a large displacement per half cycle. Choosing the frequency well below that of the mechanical droplet resonance retains the advantage of AC signals, while effectively presenting a DC signal: the droplet has enough time to adapt to the slowly changing voltage. This also means that the highest effective voltage is now the peak voltage itself, instead of the RMS voltage, which is $\sqrt{2}$ times smaller.

Indeed, application of an AC frequency of $50 \mathrm{~Hz}$ resulted in stronger deflections of the contact line; close inspection of video frames taken at $7000 \mathrm{fps}$ revealed that the right- 
hand side contact line of the droplet showed periodic motion in the direction of the activated electrodes. The frequency of this oscillation was $100 \mathrm{~Hz}$, in agreement with expectations: within each $\mathrm{AC}$ cycle, the condition where the two electrodes have the same potential, occurs twice. A few cycles were sufficient to release the drop. As a mechanistic explanation we propose that the sinusoidal deflection of the contact line (See Fig. 4-6c) at the lower side of the drop is transmitted to the upper side, resulting in depinning from the patch. The release process is also illustrated in Fig. 4-6d.

Remarkably, just after being released from the patch the droplet oscillates at $50 \mathrm{~Hz}$ between the active and grounded electrodes (see Movie 4-3). Since the EW / DEP electrostatic force depends on the square of the applied potential, it would shake the droplet at $100 \mathrm{~Hz}$. Moreover this force would tend to center and stretch the droplet, which is also in contradiction with the observations.

Coulomb forces caused by a (positive or negative) net charge of the droplet, would produce an alternating motion at the frequency at which the electrode potentials are
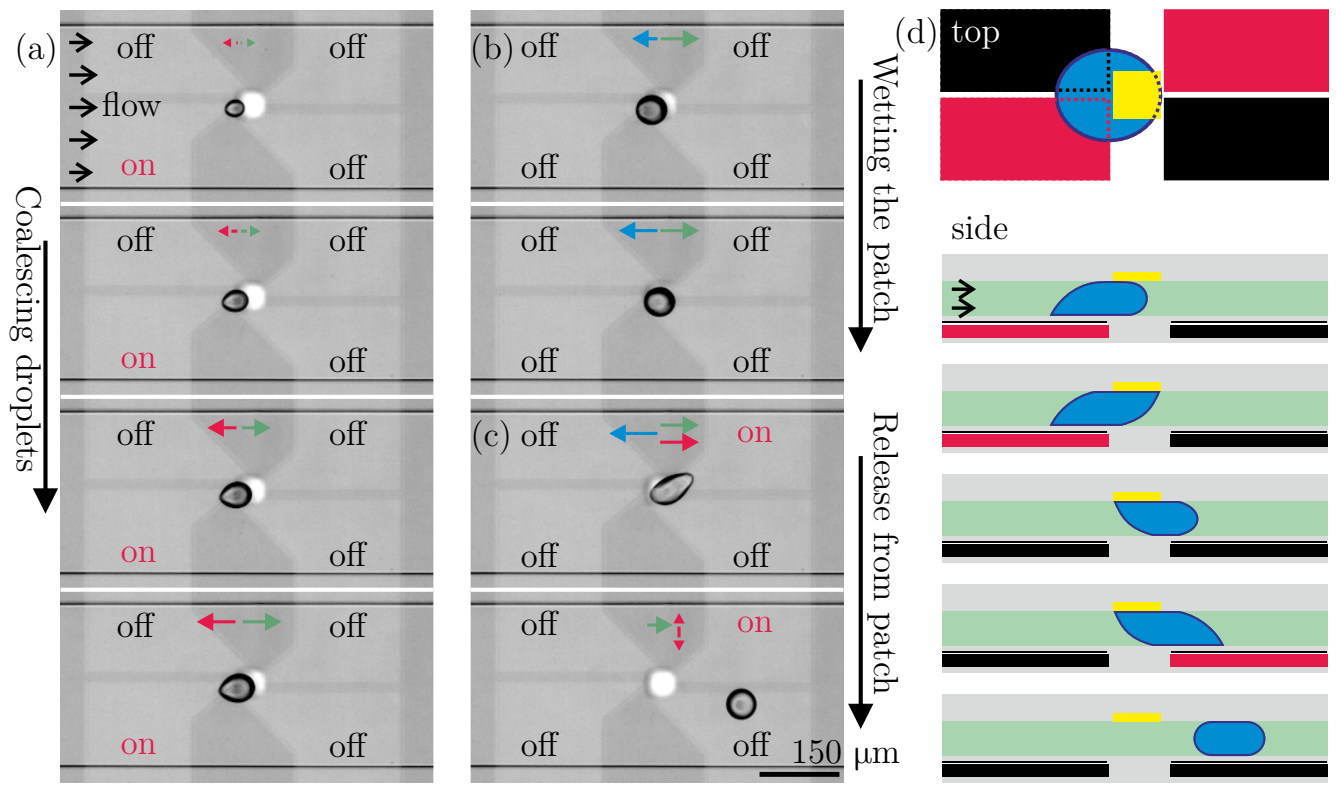

Fig. 4-6 Microscopic top view of the steps during trapping and release of a droplet on a hydrophilic patch. Oil flow rate $285 \mu \mathrm{L} / \mathrm{h}$. (a) Trapping of droplets with electrodes (300 $V, 4 \mathrm{kHz}$ ). Four consecutive droplets coalesce to form a larger droplet. (b) The electrodes are turned off. The droplet now slowly spreads over the hydrophilic patch. (c) The release electrodes are activated $(300 \mathrm{~V}, 50 \mathrm{~Hz})$ and the droplet is pulled off the patch. Hereafter the droplet oscillates at $50 \mathrm{~Hz}$ between the electrodes. (d) Schematic representation (top and side views) of the experiment. In the side views the droplet gets trapped by electrodes, wets the patch, remains adhered to the patch after the trap is switched off, and gets pulled of the patch by electrodes. Movie 4-3 shows a recording of the experiment. 
switched between positive and negative, and thus produce the observed $50 \mathrm{~Hz}$. Since no indications were obtained that the droplets were charged during trapping and pinning, the implication would be that charges are introduced during the interaction with the pulloff electrodes. The formation of charged droplets by EW electrodes is known to occur in scenarios where the droplet is so quickly split in two parts, that the separated (counter) charges residing near the two EW electrodes do not have sufficient time to recombine. ${ }^{38}$ Thinking along this line, it might be that during the detaching of the drop, a tiny aqueous volume (too small to observe microscopically) with a residual charge is left behind (with the counter charge residing in the released drop). Another possibility would be that electrically charged satellite droplets are ejected from the main droplet, similar to our observations at high voltages in Section 4.5.1.

For a typical experiment as shown in Fig. 4-6 the relevant forces can be estimated, using equations 4.3 and 4.5 for the drag and pinning forces, and a numerical calculation of the electrostatic force (see Appendix 4B). Additional measurements were done to complete the set of input parameters: a flow velocity $u_{\text {oil }}$ of $8.5 \mathrm{~mm} / \mathrm{s}$ (approximated at the centerline of the channel), an oil-water interfacial tension $\gamma_{\text {ow }}$ of $50 \mathrm{mN} / \mathrm{m}$, a patch width of $45 \mu \mathrm{m}$ and water contact angles on the Cytop $\theta_{\text {phobic }}$ of $160^{\circ}$ and a receding angle on the MUA $\theta_{\text {philic }}$ of $50^{\circ}$ (see Appendix $4 \mathrm{C}$ ). This resulted in:

$$
F_{d} \approx 0.33, F_{\text {wet }} \approx 3.6, \quad F_{\text {el, } \max } \approx 3.2
$$

These numbers, which have to be considered as crude estimates, corroborate that the maximum electrostatic force is larger than the drag force, and that droplets can be trapped with electrodes. Similarly, the pinning force is found to be larger than the drag force, in agreement with our observation that droplets remain on the patch in spite of the flow. Finally, the resultant force of the drag and electrowetting is about as large as the pinning force, explaining the difficulty in removing the droplet from the patch.

\subsection{Discussion}

\subsubsection{Fabrication}

In the search for a microfluidic device that contains electrodes as well as hydrophilic patches many different fabrication protocols have been explored. Finally, we have found a protocol that - with some practice on the experimenter's side - generates a well-aligned, bi-functional, two-sided microchip nearly every time. Several key aspects to this improved fabrication process have to be mentioned. First, the use of $3 \mu \mathrm{m}$ higher pillars around the channel mold automatically ensures the presence of a thin dielectric layer of PDMS atop the electrodes. This simplifies the process and saves time and effort compared to other protocols to create a thin, homogenous dielectric, like separately spinning a PDMS layer, evaporating parylene-c, or dipcoating Teflon AF atop the electrodes. Second, since the electrodes and channel are pressed together with liquid PDMS, the mold and electrodes can be moved with respect to each other even after pressing them together. This ensures a near-perfect alignment. Specifically, because, third, the in-situ curing of the liquid 
PDMS by use of a Peltier element on the stereomicroscope ensures that the chip halves do not misalign before curing is complete (for instance during transport to an oven). Fourth, by using Cytop as a sturdy hydrophobic material for the hydrophilic patch side, instead of for instance Teflon AF, it is now possible to use a shadow mask without damaging the hydrophobic layer. This simplifies the local removal of Cytop by oxygen plasma and the sputtering of gold, without the need for additional photolithographic steps. Also the specific bonding of mercapto-undecanol to gold allows to rinse the Cytop effortlessly, creating a hydrophobic substrate with low contact angle hysteresis around the hydrophilic patches. The elasticity of the $55 \mu \mathrm{m}$ PDMS layer allows to seal the chip, as long as pressure is applied homogeneously around the channel. For ease of use, in this setup magnets provided this pressure, which formed a leak-free seal for the oil flowrates and oil type used in this paper.

\subsubsection{Operation}

As for qualitative functionality, the microchip worked as expected. Trapping electrodes are needed to allow for the passive breakup of the thin oil film and attachment of the droplet to the hydrophilic patch. Even though this process takes less than a few seconds, other applications might require wetting to occur faster. In that case it would be possible to place the hydrophilic patch on the electrode chip half. The electrostatic force would then actively pull the droplet towards the hydrophilic patch, ensuring an active squeezeout of the oil film within several milliseconds. ${ }^{30}$

After the droplet wets the patch, the pinning force is larger than the drag force, as indicated by the fact that the droplet in flow remains on the patch for prolonged periods of time. This also confirms the need for release electrodes to pull the droplet off the patch.

The trapping/pinning/release experiments were successfully performed multiple times for droplets consisting of 2, 3, 4 and 5 merged primary droplets. Using just one primary droplet only succeeded if the droplet was not given enough time to completely spread over the patch. We can explain this by the fact that the pinning force for a droplet spread over the patch is determined by the width of the patch (independent of droplet size), while the electrostatic and drag force are determined by the radius of the droplet. Likely due to the non-optimal choice of electrode geometry it proved to be impossible to drag the droplet from the patch using high frequency actuation only. The shift to a low frequency actuation signal finally enabled the release of droplets of radius 19 to $31 \mu \mathrm{m}$ from the patch.

The novel functionality of our device has several advantages over previous combinations of electrowetting and SPR. Compared to only using electrowetting for all droplet manipulations, ${ }^{20}$ the addition of two-phase flow microfluidics helps in the release of a droplet from a patch. Furthermore, microfluidics enables the high speed generation of picoliter sized droplets, ${ }^{18}$ which for instance can contain single cells. ${ }^{39}$ These small volumes are required to quickly reach sufficient concentrations of cell-excreted molecules inside the droplets to enable SPR detection.

We supply formulas for the estimation of the forces related to our work, which are helpful guidelines for designing a microfluidic chip combining wetting patches and electrowetting. 


\subsection{Conclusions and Outlook}

We successfully designed and fabricated a multifunctional, two sided microchip capable of forcing picoliter sized droplets to adhere to an artificial wetting defect, and thereafter control the release of the droplet to flow towards a next measurement site. This demonstrates the capability of our technique for future implementation with measurement techniques that require surface molecular interaction.

In the trapping experiments we observed that the EW and drag forces scale in the expected way with electric field and drop radius. Although quantitative agreement with the used (approximative) theoretical expressions is not achieved, large enough EW trapping forces could be generated, by adapting the voltage to the drop size and flow rate.

Under (our) flow conditions, wetting of the hydrophilic patch by the aqueous drop does not occur spontaneously, even though it is thermodynamically favored. This is ascribed to the presence of an oil film, which does not have sufficient time to be squeezed out. However, using EW forces it was possible to hold the drop long enough under the patch, for wetting to occur.

The pinning forces from the patch turned out to be strong enough to resist the drag force and hence immobilize the drop. Pull-off from the patch could be achieved with the designated electrodes, however not via EW alone. This can be rationalized with our approximate calculations, which indicate that the pinning and EW forces are of comparable magnitude. This limitation is however not inherent. It could be overcome via an improved design of the electrode geometry.

In the current design only three electrode connections were used to create multiple trapping and release stations. This is efficient, but also poses limitations to the EW force. Use of individually addressable electrode pairs (see Fig. 4-6d) per trapping and release geometry would take away this limitation.

Our device should be well suited for studies of cells in droplets (or cell supernatants) with SPRi, where it is desirable to perform multiplex analysis of the same droplet on subsequent sensor patches, and to reuse the chip after replacement of either actuation or detector surface. Exchange of $\mathrm{O}_{2}$ and $\mathrm{CO}_{2}$ produced by the cell during this time is favored by the relatively large amount of oil surrounding the drops. Enhancement of the throughput could be achieved via parallelization, i.e. the use of multiple traps side by side. This is straightforward to accomplish, as we have previously shown in de Ruiter, et al. $(2014)^{26}$, where droplets were laterally guided and trapped atop multiple arrays of electrodes. This principle can be adapted for the use of multiple trapping and release geometries as well. 


\section{Appendix 4A: cleanroom masks for chip fabrication}

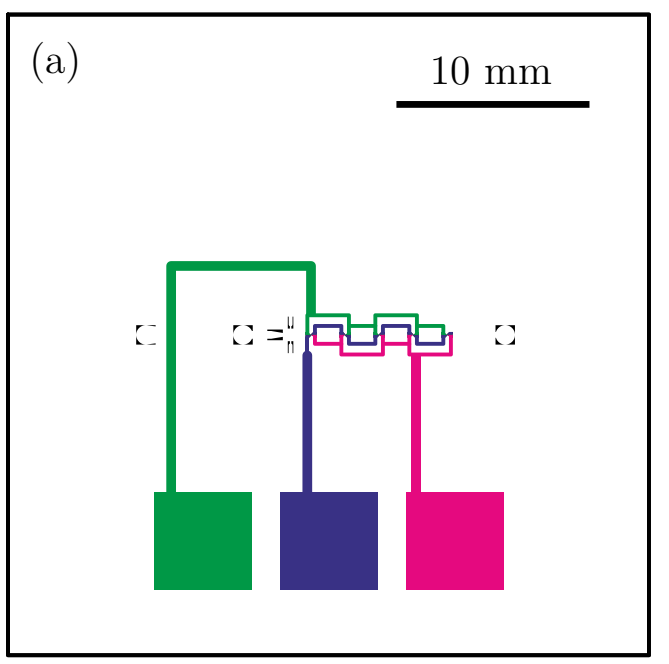

(b)

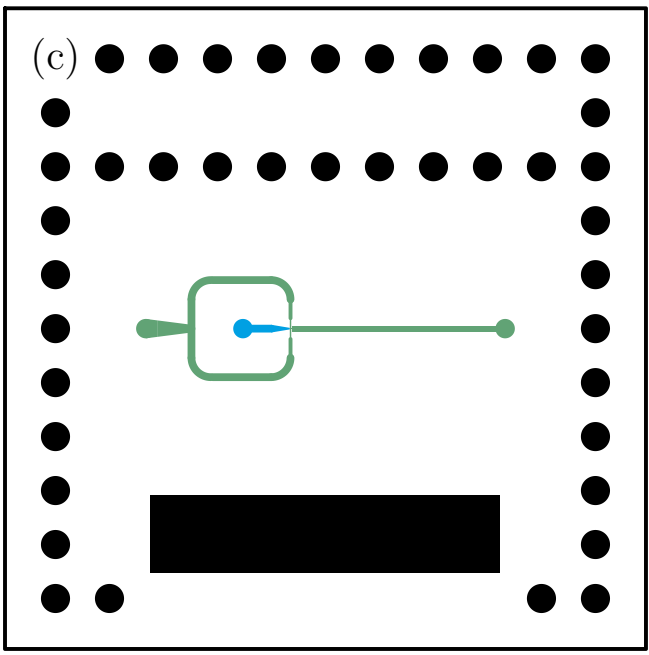

(a) Mask for ITO electrode structure. The 3 main electrodes forming trapping and release geometry. The different colors illustrate the different electrodes. Black are the alignment markers in the ITO. (b) Green: mask for the powderblasted inlets and electrode access in the top glass plate, Yellow: mask for the creation of the shadow mask in silicon for etching of Cytop and the deposition of the gold patches. Tiny black squares in the center of the yellow squares are the actual gold patch sizes (only visible in digital version). (c) Masks for the channel structure and surrounding $3 \mu \mathrm{m}$ higher pillars. Colors only indicate which part will be for water (blue) or oil (green). Black is present in the original mask with channel, as well as a secondary mask for only increasing the pillar height. 


\section{Appendix 4B: determining the trapping force}

(a)

(b)
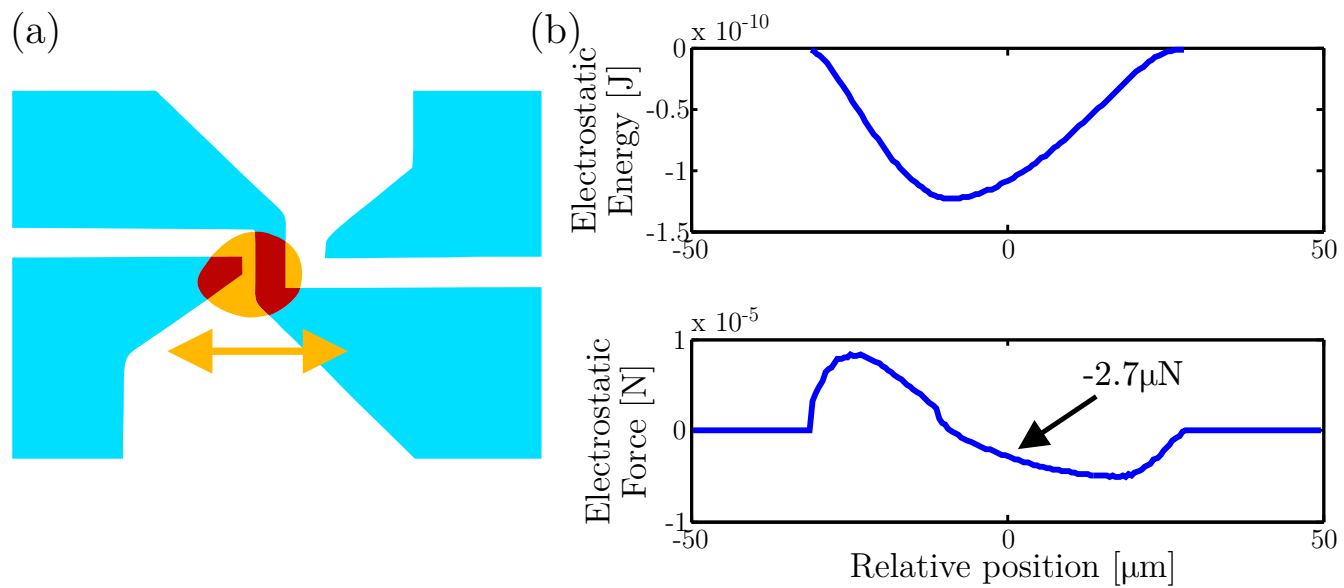

Determination of the electrostatic energy and derivation of the electrostatic force. (a) Image analysis of measured droplet being trapped. Light blue: electrode geometry. Orange: droplet area not above electrode. Red: droplet area above electrodes. The orange arrows indicates the direction in which the droplet area is artificially translated to determine the electrostatic energy based on the area of grounded and active electrode covered by the droplet. (b) Top: The calculated electrostatic energy for a droplet translated w.r.t. the trapping position. Only around 0 the data is valid, but the entire graph does show the standard shape of an electrostatic potential well. Bottom: The numerically determined electrostatic force from the derivative of the electrostatic energy. Again, only around 0 the data is relevant, but the entire graph does show the standard electrostatic force curve associated with a droplet moving on and off 2 electrodes. Around 0 the force is $-2.7 \mu N$.

\section{Appendix 4C: CA measurements for Cytop and MUA/gold}
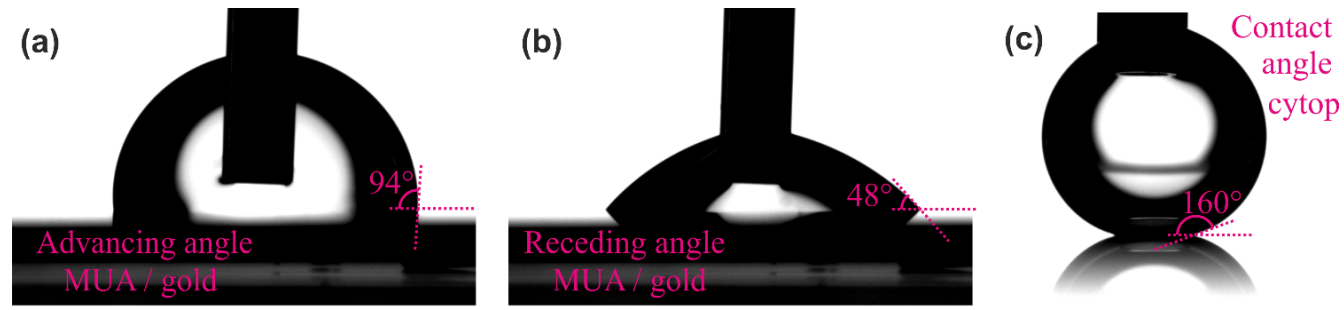

$(\boldsymbol{a}, \boldsymbol{b})$ Advancing and receding contact angle (ARCA) measurements of water on top of hydrophilized gold in light mineral oil. Results: $\boldsymbol{\theta}_{\mathbf{a d v}} \sim \mathbf{9 4}^{\circ}, \boldsymbol{\theta}_{\mathbf{r e c}} \sim \mathbf{4 8}^{\circ}$. (c) For water atop Cytop under light mineral oil the contact angles are $\boldsymbol{\theta}_{\mathbf{a d v}} \sim \mathbf{1 6 4}^{\circ}, \boldsymbol{\theta}_{\text {rec }} \sim \mathbf{1 5 7}^{\circ}$. 


\section{Appendix 4D: Snapshots of droplet coalescence}

The coalescing of multiple droplets generated on demand with a pressure pulse to the water inlet. See also first part of Movie 4-3.

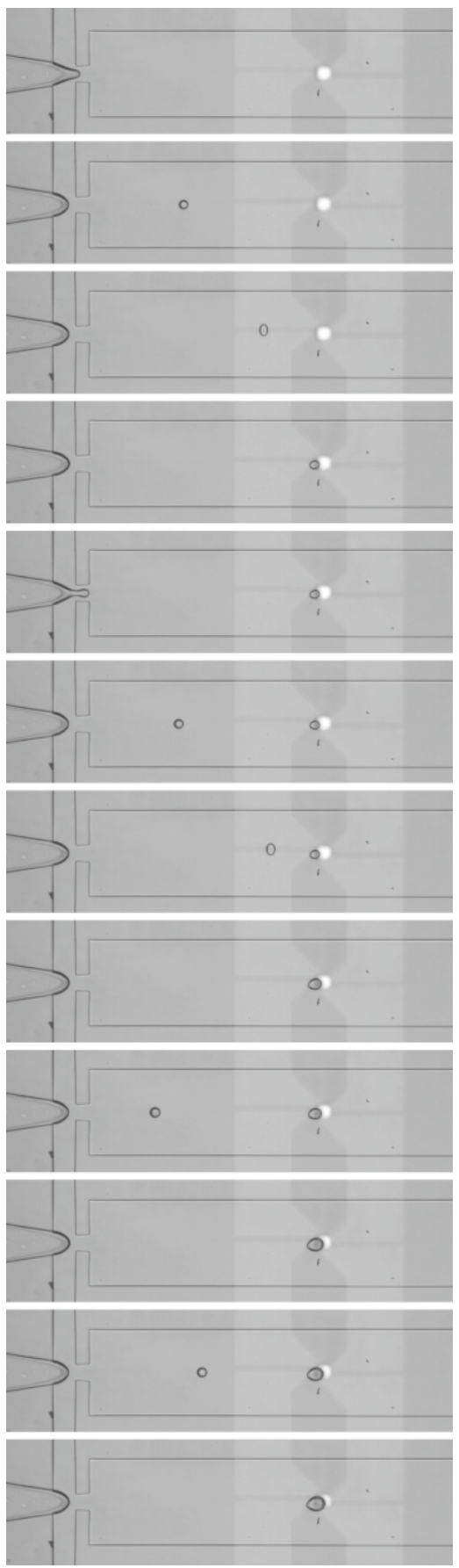

Generation of first droplet

First droplet dragged along the channel

Guided towards the end of the trap

One drop trapped by electrowetting

Generation of second droplet

Second droplet dragged along the channel

Guided towards the end of the trap

Two drops merged and trapped

Third droplet dragged along the channel

Three drops merged and trapped

Fourth droplet dragged along the channel

Four drops merged and trapped 


\section{References}

1. Casadevall i Solvas, X. and A. deMello, Droplet microfluidics: recent developments and future applications. Chemical Communications, 2011. 47(7): p. 1936-42.

2. Seemann, R., M. Brinkmann, T. Pfohl, et al., Droplet based microfluidics. Reports on Progress in Physics, 2012. 75(1).

3. Nisisako, T. and T. Torii, Microfluidic large-scale integration on a chip for mass production of monodisperse droplets and particles. Lab Chip, 2008. 8(2): p. 287-93.

4. Calvert, P., Inkjet Printing for Materials and Devices. Chemistry of Materials, 2001. 13(10): p. 3299-305.

5. Margulies, M., M. Egholm, W.E. Altman, et al., Genome sequencing in microfabricated highdensity picolitre reactors. Nature, 2005. 437(7057): p. 376-80.

6. Song, H., D.L. Chen, and R.F. Ismagilov, Reactions in droplets in microfluidic channels. Angew Chem Int Ed Engl, 2006. 45(44): p. 7336-56.

7. Guo, M.T., A. Rotem, J.A. Heyman, et al., Droplet microfluidics for high-throughput biological assays. Lab on a Chip, 2012. 12(12): p. 2146-55.

8. Theberge, A.B., F. Courtois, Y. Schaerli, et al., Microdroplets in Microfluidics: An Evolving Platform for Discoveries in Chemistry and Biology. Angewandte Chemie-International Edition, 2010. 49(34): p. 5846-68.

9. Takinoue, M. and S. Takeuchi, Droplet microfluidics for the study of artificial cells. Analytical and Bioanalytical Chemistry, 2011. 400(6): p. 1705-16.

10. Kuswandi, B., Nuriman, J. Huskens, et al., Optical sensing systems for microfluidic devices: a review. Anal Chim Acta, 2007. 601(2): p. 141-55.

11. Erickson, D., S. Mandal, A.H.J. Yang, et al., Nanobiosensors: optofluidic, electrical and mechanical approaches to biomolecular detection at the nanoscale. Microfluidics and Nanofluidics, 2008. 4(1-2): p. 33-52.

12. Mairhofer, J., K. Roppert, and P. Ertl, Microfluidic Systems for Pathogen Sensing: A Review. Sensors, 2009. 9(6): p. 4804-23.

13. Stojanovic, I., R.B. Schasfoort, and L.W. Terstappen, Analysis of cell surface antigens by Surface Plasmon Resonance imaging. Biosens Bioelectron, 2014. 52: p. 36-43.

14. Rich, R.L. and D.G. Myszka, Higher-throughput, label-free, real-time molecular interaction analysis. Anal Biochem, 2007. 361(1): p. 1-6.

15. Stojanovic, I., T.J. van der Velden, H.W. Mulder, et al., Quantification of antibody production of individual hybridoma cells by surface plasmon resonance imaging. Anal Biochem, 2015. 485: p. 112-8.

16. Chen, J.S. and J.H. Jiang, Droplet Microfluidic Technology: Mirodroplets Formation and Manipulation. Chinese Journal of Analytical Chemistry, 2012. 40(8): p. 1293-300.

17. Cho, S.K., H.J. Moon, and C.J. Kim, Creating, transporting, cutting, and merging liquid droplets by electrowetting-based actuation for digital microfluidic circuits. Journal of Microelectromechanical Systems, 2003. 12(1): p. 70-80.

18. Pit, A.M., M.H.G. Duits, and F. Mugele, Droplet Manipulations in Two Phase Flow Microfluidics. Micromachines, 2015. 6(11): p. 1768-93.

19. Luo, Y., F. Yu, and R.N. Zare, Microfluidic device for immunoassays based on surface plasmon resonance imaging. Lab Chip, 2008. 8(5): p. 694-700. 
20. Malic, L., T. Veres, and M. Tabrizian, Two-dimensional droplet-based surface plasmon resonance imaging using electrowetting-on-dielectric microfluidics. Lab on a Chip, 2009. 9(3): p. $473-5$.

21. Malic, L., T. Veres, and M. Tabrizian, Nanostructured digital microfluidics for enhanced surface plasmon resonance imaging. Biosens Bioelectron, 2011. 26(5): p. 2053-9.

22. Yeatman, E.M., Resolution and sensitivity in surface plasmon microscopy and sensing. Biosensors \& Bioelectronics, 1996. 11(6-7): p. 635-49.

23. Bithi, S.S., W.S. Wang, M. Sun, et al., Coalescing drops in microfluidic parking networks: A multifunctional platform for drop-based microfluidics. Biomicrofluidics, 2014. 8(3): p. 034118.

24. Fradet, E., C. McDougall, P. Abbyad, et al., Combining rails and anchors with laser forcing for selective manipulation within 2D droplet arrays. Lab Chip, 2011. 11(24): p. 4228-34.

25. Leung, K., H. Zahn, T. Leaver, et al., A programmable droplet-based microfluidic device applied to multiparameter analysis of single microbes and microbial communities. Proc Natl Acad Sci U S A, 2012. 109(20): p. 7665-70.

26. de Ruiter, R., A.M. Pit, V.M. de Oliveira, et al., Electrostatic potential wells for on-demand drop manipulation in microchannels. Lab Chip, 2014. 14(5): p. 883-91.

27. Baret, J.C., O.J. Miller, V. Taly, et al., Fluorescence-activated droplet sorting (FADS): efficient microfluidic cell sorting based on enzymatic activity. Lab on a Chip, 2009. 9(13): p. 1850-8.

28. Schmid, L., D.A. Weitz, and T. Franke, Sorting drops and cells with acoustics: acoustic microfluidic fluorescence-activated cell sorter. Lab Chip, 2014. 14(19): p. 3710-8.

29. Pit, A.M., R. de Ruiter, A. Kumar, et al., High-throughput sorting of drops in microfluidic chips using electric capacitance. Biomicrofluidics, 2015. 9(4): p. 044116.

30. Staicu, A. and F. Mugele, Electrowetting-induced oil film entrapment and instability. Phys Rev Lett, 2006. 97(16): p. 167801.

31. Cavalli, A., M. Musterd, and F. Mugele, Numerical investigation of dynamic effects for sliding drops on wetting defects. Phys Rev E Stat Nonlin Soft Matter Phys, 2015. 91(2): p. 023013.

32. 't Mannetje, D., S. Ghosh, R. Lagraauw, et al., Trapping of drops by wetting defects. Nat Commun, 2014. 5: p. 3559.

33. 't Mannetje, D., A. Banpurkar, H. Koppelman, et al., Electrically tunable wetting defects characterized by a simple capillary force sensor. Langmuir, 2013. 29(31): p. 9944-9.

34. Schoeman, R.M., E.W. Kemna, F. Wolbers, et al., High-throughput deterministic single-cell encapsulation and droplet pairing, fusion, and shrinkage in a single microfluidic device. Electrophoresis, 2014. 35(2-3): p. 385-92.

35. Gu, H., C.U. Murade, M.H. Duits, et al., A microfluidic platform for on-demand formation and merging of microdroplets using electric control. Biomicrofluidics, 2011. 5(1): p. 11101.

36. Mugele, F. and J.-C. Baret, Electrowetting: from basics to applications. Journal of Physics: Condensed Matter, 2005. 17(28): p. R705.

37. Li, F. and F. Mugele, How to make sticky surfaces slippery: Contact angle hysteresis in electrowetting with alternating voltage. Applied Physics Letters, 2008. 92(24).

38. Baret, J.C. and F. Mugele, Electrical discharge in capillary breakup: controlling the charge of a droplet. Phys Rev Lett, 2006. 96(1): p. 016106.

39. Chabert, M. and J.L. Viovy, Microfluidic high-throughput encapsulation and hydrodynamic self-sorting of single cells. Proc Natl Acad Sci U S A, 2008. 105(9): p. 3191-6. 


\section{5 \\ High throughput sorting}

We analyze the new approach, as described in Chapter 3, for the sorting of aqueous drops with biological content immersed in oil, using a microfluidic chip that combines the functionality of electrowetting with the high throughput of twophase flow microfluidics. In this electrostatic sorter, three co-planar electrodes covered by a thin dielectric layer are placed directly below the fluidic channel. Switching the potential of the central electrode creates an electrical guide that leads the drop to the desired outlet. The generated force, that deflects the drop, can be tuned via the voltage. The working principle is based on a contrast in conductivity between the drop and the continuous ambient phase, which ensures successful operation even for drops of highly conductive biological media like phosphate buffered saline. Moreover, since the electric field does not penetrate the drop, its content is protected from electrical currents and Joule heating. A simple capacitive model allows quantitative prediction of the electrostatic forces exerted on drops. The maximum achievable sorting rate is determined by a competition between electrostatic and hydrodynamic forces. Sorting speeds up to 1200 per second are demonstrated for conductive drops of $160 \mathrm{pL}$ in low viscosity oil.

This chapter has been published as:

A.M. Pit, R. de Ruiter, A. Kumar, D. Wijnperlé, M.H.G. Duits and F. Mugele, "Highthroughput sorting of drops in microfluidic chips using electric capacitance", Biomicrofluidics 9(4): p. 044116 (2015) 


\subsection{Introduction}

In the past decade droplet microfluidics has matured from "having great potential" to real-life applications. ${ }^{1}$ Small drops used as micro reactors require minute amounts of reaction material while fast drop generation allows for numerous samples in a short time. A topic of high interest in the field is the analysis of biological cells encapsulated in drops. Drops provide an attractive means to separate a cell from its surroundings and assess its individual characteristics. These include not only markers that are localized on the cell membrane, but also concentrations of biomolecules excreted by the living cell or released after cell lysis. Such applications often involve large populations (thousands to millions) of cells, implying the generation and inspection of at least an equal number of drops. While significant progress has been made in handling such staggering numbers of drops, ${ }^{2}$ there are also several issues remaining that still need to be addressed.

One of these issues is obtaining populations of drops which all contain one cell. Simply generating drops from a cell suspension leads to distributions in the occupancy per drop: empty, single, double, etc. due to the inherent Poisson statistics. The concentration of the cell suspension and the volume of the drops then determine the relative probabilities. ${ }^{3}$ One approach to circumvent this issue has been to passively arrange the cells before injection. Techniques like close-packed ordering ${ }^{3}$ and Dean flow ordering ${ }^{4}$ have been applied with some success, but do require fine-tuning of oil flows and the final cell-in-drop population is not $100 \%$ for prolonged periods of time.

An alternative, and potentially more reliable, approach is to actively sort out drops that contain only one cell. To this end several techniques have been developed, for instance sorting based on contrast in permittivity (dielectrophoresis, DEP), ${ }^{5,6}$ the use of surface acoustic waves $(\mathrm{SAW}),{ }^{7}$ using pressure pulses,${ }^{8}$ electrically pre-charging the drops, ${ }^{9}$ laser induced sorting, ${ }^{10}$ and the use of membrane valves. ${ }^{11} \mathrm{~A}$ drawback of all these methods is that the number of drops to be sorted is even higher than the already staggering number of cells: in a typical injection of a cell suspension, the average occupancy per drop is often in the range 0.1-0.2. Clearly, this prompts the need for high sorting speed devices.

A second issue concerns the reliability of the drop manipulation. Even if each of the drops already contains one cell, they still need to be analyzed at a specific on-chip location, or transported to a specific outlet with high probability of success, and without damage along the path.

In Chapter 3, we presented a drop manipulation method, which combines the functionality of electrowetting-on-dielectric (EWOD) with the high throughput of twophase flow microfluidics by integrating insulated electrode geometries just below the microchannel. The method can, besides trapping, releasing, splitting and guiding, also perform sorting of drops in flow. ${ }^{12}$ It is highly promising, because of, $i$ ) on-demand and fast actuation: electric fields can be switched rapidly, unlike mechanical valves or hydrostatic pressures, $i i$ ) non-invasiveness: the electric field does not penetrate the drop and therefore causes no (potentially harmful) Joule heating, iii) ease and flexibility of design, $i v$ ) ability to predict the electrostatic force based on a capacitive model. This technique, however, only showed sorting rates of 25 drops per second and has not been translated to a practical drop sorter. 
The present chapter focuses on the reliability and speed of sorting, while also durability and bio-compatibility are considered. In particular, we discuss the capabilities of a sorter similar to one that was recently introduced ${ }^{12}$ and has since then been improved to allow sorting at 1200 drops per second. The performance of the sorter is characterized. First, the theoretical dependence of the sorting force on the actuation voltage and drop size is explored. We show that the electrostatic and hydrodynamic forces on the drop can be estimated with moderate accuracy from analytical models, and used to predict the required sorting conditions. Subsequently, several working points around this condition are explored, to ascertain the quantitative requirements for $>99 \%$ sorting efficiency.

We demonstrate the performance of our sorter via different experiments. We show that the use of highly conductive liquids, like phosphate buffered saline, do not pose any limitations to the ability to sort drops; thus opening the road to its use for biological applications. We show that sorting speeds as high as 1200 drops per second can be achieved by using a low viscosity oil. Finally, we also report preliminary results of active sorting on demand, using fluorescence based detection of cells in drops, achieving a rate of 400 drops per second.

\subsection{Materials and Methods}

The setup for generating and sorting drops in ambient oil is illustrated in Fig. 5-1. In a $50 \mu \mathrm{m}$ high microchannel, drops are generated via a flow focusing device (FFD) with a $50 \mu \mathrm{m}$ wide orifice. Water drops are formed in the continuous oil phase consisting of Fluorinert FC-40. The generated drops are squeezed between the top and bottom wall of the microchannel. The channel itself is $200 \mu \mathrm{m}$ wide.

The pressures of the oil and water phases are separately adjusted by a custom written Labview program, which drives a home-made pressure controller consisting of two pressure regulators and three-way solenoid valves. The air pressure difference $(\Delta P)$ of the two closed vials containing water or oil can be independently regulated from 1-100 $k P a$. The drop radius, speed and inter-drop distance can be tuned via the magnitudes and ratio of the oil and water phase pressures. The oil flow rate can be set from 0 to $\sim 10 \mathrm{~mL} / \mathrm{h}$, corresponding to average velocities of 0 to $\sim 40 \mathrm{~cm} / \mathrm{s}$ in the main channel. The drop radii can be varied from around 25 to $60 \mu \mathrm{m}$, and the drop generation frequency $f_{\text {drop }}$ can be altered from 0 to 2500 drops per second. The pressure controller allows to create single drops on demand by applying a pressure pulse of chosen duration $(\Delta t)$ and magnitude $(\Delta P)$ to the water phase, or it can create drops continuously by setting an overpressure to the water phase.

An inverted microscope in combination with a high speed camera is used to visualize and record the sorting events. Matlab scripts are used to identify which outlet channel is taken by individual drops, and to extract parameters like drop radius, inter-drop distance, drop generation frequency and drop velocity from the video recordings. We also measure the position dependent overlap of the droplet with each electrode.

In Fig. 5-2a and b a topview of the microchip is shown. Polydimethylsiloxane (PDMS) microchannels are molded using standard soft lithography techniques. ${ }^{13}$ Electrode patterns are etched in an indium tin oxide (ITO) coated glass substrate using an $18 \% \mathrm{HCl}$ solution. 


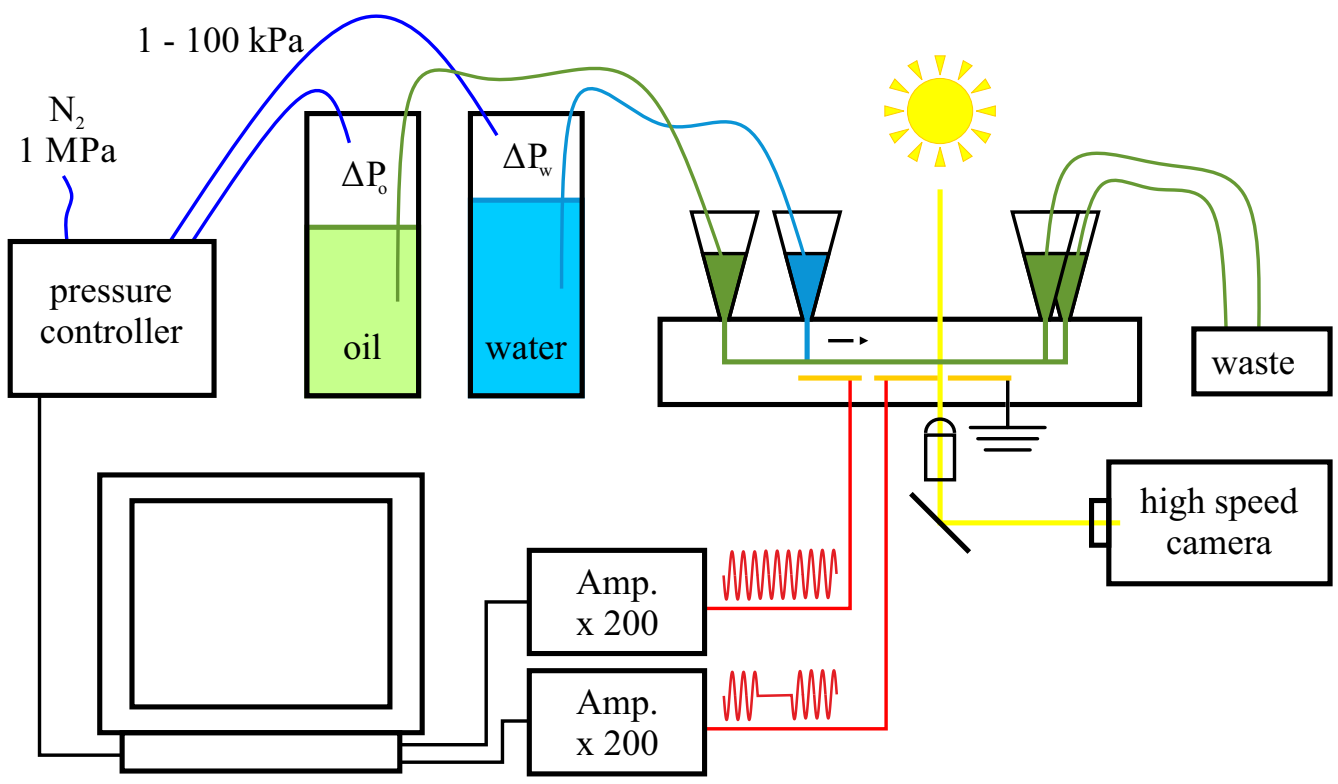

Fig. 5-1 Schematic overview of the experimental setup. DAq cards in the computer send two sine waves to two amplifiers attached to the electrodes on the microchip. The computer also controls the pressure of two closed vials - one containing water, the other oil - which allows to control the drop generation and flow rates at the flow focusing device in the chip. A microscope and high speed camera are used to capture the drops passing the electrodes in the channel at 5000 fps.

Atop the electrodes a PDMS layer of approximately $3 \mu \mathrm{m}$ is created by spin coating 600 $\mu L$ of PDMS at $6000 \mathrm{rpm}$ for $600 \mathrm{~s}$. This thin layer is partially cured at $65^{\circ} \mathrm{C}$ for $20 \mathrm{~min}$. Using a stereomicroscope, the electrode structure with partially cured layer is aligned with a previously fabricated PDMS microchannel. After alignment, the curing process is completed at $65^{\circ} \mathrm{C}$ for another hour to form a permanent seal between the channel and electrode substrate. The thin PDMS layer not only helps sealing the chip, but also serves as the dielectric required to insulate the drop from the electric currents.

Sorting takes place $3 \mathrm{~mm}$ downstream from the FFD where a set of 3 electrodes is aligned underneath the channel in front of a symmetric Y-shaped channel junction. The gap between the electrodes was designed to be $10 \mu m$, i.e. significantly larger than twice the thickness of the dielectric layer, to prevent short circuiting. Drops are guided towards the desired outlet by switching the center electrode. This is done via a custom made Labview program that generates two in-phase AC sine waves. One of the generated AC signals is modulated with a square pulse (from 0 to 1 ) for a duration of $1 / f_{\text {drop }}$, effectively switching the signal between grounded and active state. This modulated wave is sent towards the switching electrode. The unmodulated signal is continuously sent to the bottom electrode, which is always active. Both signals are pre-amplified 200-fold by an AC amplifier. The top electrode is continuously grounded (Fig. 5-2b). 


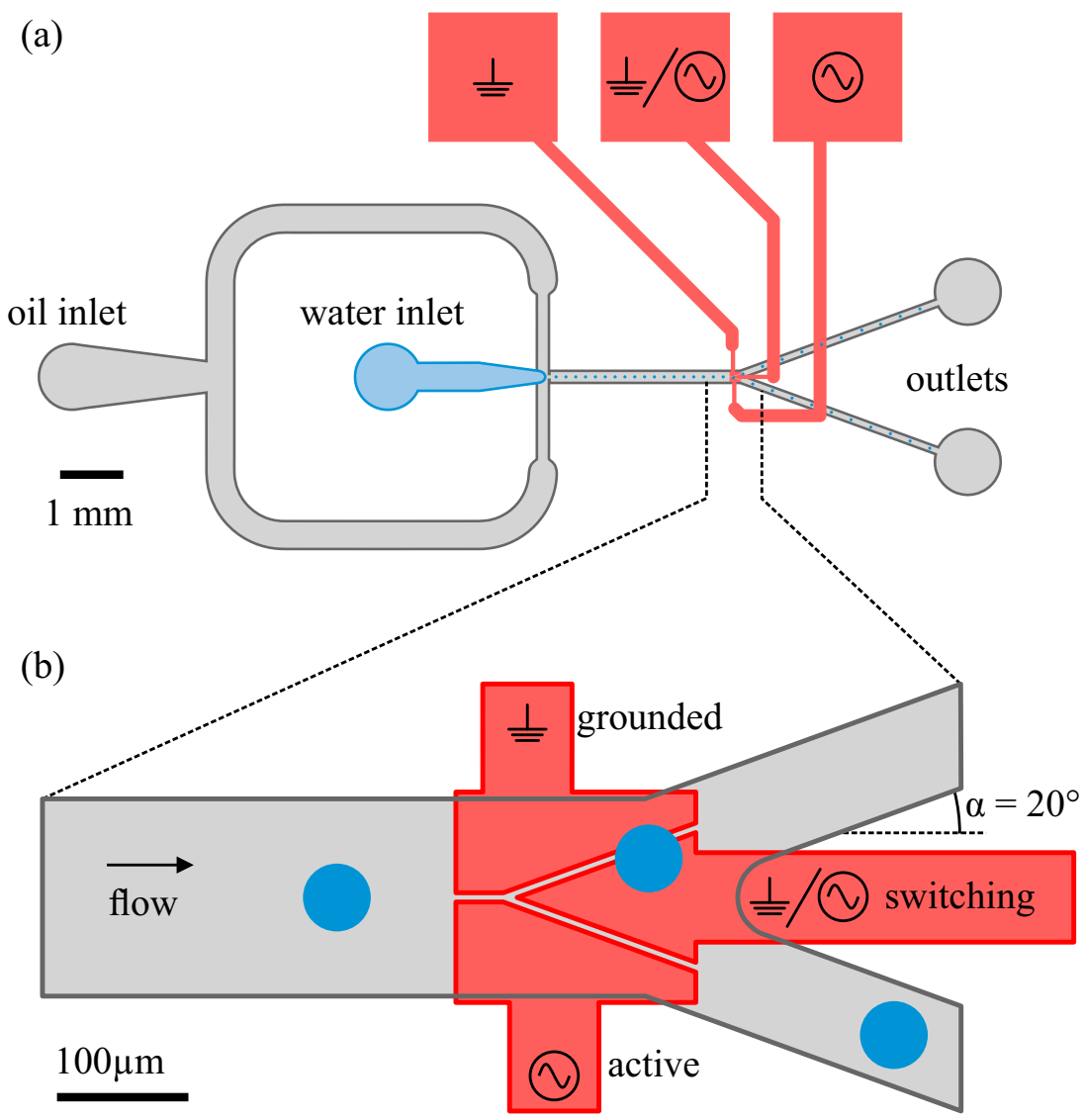

Fig. 5-2 (a) schematic overview of the microchannel geometry and the electrode structures incorporated beneath it. (b) detailed view of the sorter region showing the three-electrode geometry and which electrode is active, grounded or switching between active and ground state.

Besides the active sorting of uniform drops, which we perform to assess the capabilities and limitations of the sorter, also the sorting of drops with and without cells is explored. To this purpose, a microscope is additionally equipped with a $488 \mathrm{~nm}$ diode laser, of which the beam is expanded using a cylindrical lens and launched into the back aperture of a 40x objective, effectively illuminating a thin slice perpendicular to the channel direction. Emitted light from the fluorescently labeled cells is collected with the same objective, filtered and detected with a photomultiplier tube (PMT). A reconfigurable data acquisition card (PCIe-7842R) is used to acquire the PMT signal and generate a sorting pulse if a preset threshold is exceeded. The sorting pulse is further amplified with a highvoltage amplifier and sent to the central sorting electrode, effectively sorting single cellcontaining drops from empty drops. 


\subsection{Theory}

Our microchip functions in the low Reynolds number regime $(R e<10)$. Therefore, inertia can be neglected. Whether a drop will be sorted or not depends on the balance between the hydrodynamic drag force $F_{d r a g}$ from the surrounding oil phase and the electrostatic force $F_{e}$ caused by the voltage that is applied to the electrodes.

The working principle of our electrostatic sorter is analogous to that of the electrostatic traps previously reported for millimetric sessile drops. ${ }^{14}$ It can be easily understood by considering our co-planar electrodes as an electrical circuit consisting of two capacitors in series, as drawn in Fig. 5-3b. The electrostatic energy of a capacitor in an electric circuit that includes a voltage source is given by, ${ }^{15}$

$$
W_{c}=-\frac{1}{2} C_{t o t} U^{2}
$$

where $C_{\text {tot }}$ is the total capacitance and $U$ is the root-mean-square of the applied AC voltage. If the channel is filled only with oil, the system has a low intrinsic capacitance originating from the co-planar electrodes surrounded by the $3 \mu \mathrm{m}$ layer of PDMS and the oil. Substituting the oil above the electrodes with a conductive liquid, the representative electrical circuit changes into a set of two capacitors in series, namely the two insulating layers of PDMS. If the drop moves over the electrode gap (Fig. 5-3c) the total capacitance is dependent on the areas of the drop above each electrode, $A_{1}$ and $A_{2}$,

$$
C_{\text {tot }}=\frac{C_{1} C_{2}}{C_{1}+C_{2}}=c \frac{A_{1} A_{2}}{A_{1}+A_{2}}, \text { with } c=\frac{\epsilon_{0} \epsilon_{r}}{d},
$$

with $\epsilon_{r}$ the relative permittivity of PDMS, and $d$ the thickness of the insulating layer. For a typical water drop (with radius $R=30 \mu \mathrm{m}$ ) centered above an electrode gap of $10 \mu \mathrm{m}$, the capacitance increases from 0.7 to $4.1 \mathrm{fF}$, when the water displaces the oil. Ignoring the oil contribution, the total electrostatic energy gain as a drop moves across the electrodes (Fig. 5-3c) thus becomes:

$$
W_{e}=-\frac{1}{2} \frac{\epsilon_{0} \epsilon_{r}}{d} \frac{A_{1} A_{2}}{A_{1}+A_{2}} U_{t o t}^{2}
$$

It is therefore energetically favorable to exchange the oil with water, and this energy gain increases with the square of the applied potential. The electrostatic force that is exerted when the drop moves perpendicular to the gap can be calculated by taking the derivative with respect to the drop displacement $x$ :

$$
F_{e}=\frac{\epsilon_{0} \epsilon_{r}}{\pi d} U_{t o t}^{2} R \sin \left(\frac{\theta}{2}\right)(\pi+\sin (\theta)-\theta) \text { with } \theta=2 \cos ^{-1}\left(\frac{x}{R}\right)
$$

Here $\theta$ is the position dependent angle from the drop center with respect to the gap, as indicated in Fig. 5-3c. In Fig. 5-3a the non-dimensionalised electrostatic energy and force landscapes are plotted against the distance between the drop's center-of-mass and the center of the gap. The net electrostatic force is always perpendicular to the gap between the electrodes. In practice, this means that the drop will always try to center itself on the 
gap. External forces, like the drag force, will be opposed by this electrostatic force as the drop is pushed away from the gap, leading to either a new equilibrium position, or release from the electrodes if the external force exceeds the maximum trapping force. From the analytical formula for $F_{e}$ we can numerically determine that the maximum force is exerted when the drop is located at $x \sim 0.67 R$. Thus the electrostatic force has a maximum amplitude of

$$
F_{e l, \max }=0.58 \frac{\epsilon_{0} \epsilon_{r}}{d} U^{2} R
$$

This expression for the maximum force has been validated using a capillary force sensor. ${ }^{16}$ Previously, ${ }^{12}$ we analytically approximated the hydrodynamic drag force $F_{d}$ on a stationary drop by modelling the drop as a (cylindrical) pillar with no-slip boundary conditions in an infinitely wide channel. Since the drop diameter is about one third of the channel width $w$ we chose to neglect the effect of the channel walls on the drag force. This yields

$$
F_{d}=\left(\frac{24 \pi \mu u_{o i l} R^{2}}{h}\right)\left(1+\frac{2 K_{1}(q)}{q K_{0}(q)}\right)
$$

with $\mu$ the viscosity of the oil, $u_{\text {oil }}$ its velocity, $R$ the pillar (i.e. drop) radius, $h$ the channel height, and $K_{1}$ and $K_{2}$ modified Bessel functions of the second kind with $q=$ $2 \sqrt{3} R / h$. We also assume a constant oil velocity in the center region of the channel. For a moving drop we can then replace $u_{o i l}$ by the relative velocity $\Delta u=u_{\text {oil }}-u_{\text {drop }}$.
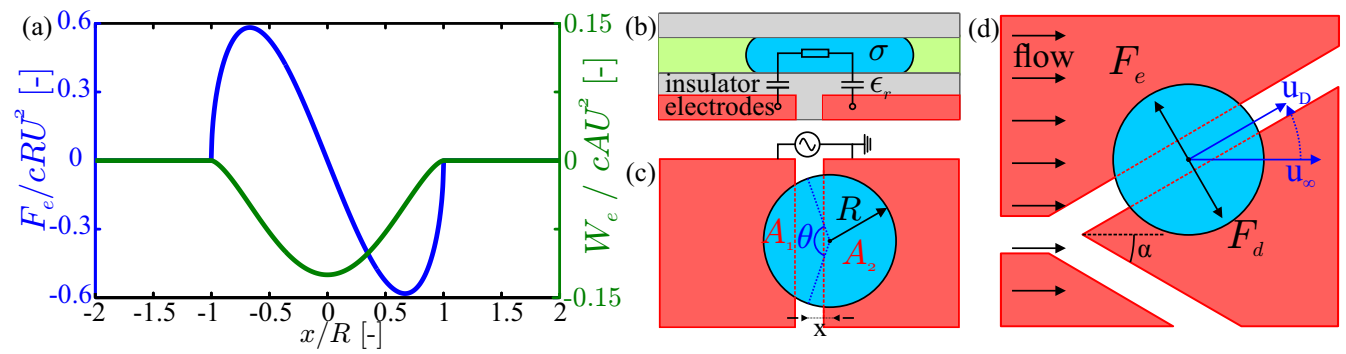

Fig. 5-3 (a) Non-dimensionalised graphs of the electrostatic potential well (green), and the resulting electrostatic force (blue) exerted on the drop as it traverses the gap, calculated from the analytical two-capacitors-in-series model, plotted against the normalized distance $x / R$, with $x$ the distance between the center of mass of the drop and the centerline of the electrode gap. (b) side view and (c) top view of a drop covering two electrodes with an applied potential difference. For conductive drops with negligible Ohmic resistance, the whole system can be considered as two capacitors in series. (d) a drop undergoing sorting. Under the influence of the electrostatic force the velocity vector of the drop decreases in magnitude and changes direction. In equilibrium, the drag force opposes the electrostatic force and the drop moves at constant velocity over the gap. 
Another factor that is of importance for the sorting operation is the inclination angle $\alpha$ of the Y-junction of the sorting electrodes (Fig. 5-3d). Before reaching the sorting geometry, the drop moves at constant velocity through the channel. The net force on the drop is zero, because viscous dissipation and drag are balanced. Under the action of the electrostatic force, the velocity vector decreases in magnitude and changes direction. The drag force adapts to this change in velocity until a new steady state is reached and the drop moves at a constant velocity over the gap between the electrodes. From a simple force balance (Fig. 5-3d), at constant drop velocity in the direction of the gap, we find that the requirement for a drop to stay on the gap is $F_{e, \max } \geq F_{d} \sin (\alpha)$. The smaller the inclination angle, the easier sorting should be. The total length of the electrode geometry is limited by the inter drop distance, because a consecutive drop should not be influenced by the voltage applied for the sorting of the previous drop. As a compromise between size and low inclination angle, an angle of 20 degrees was chosen.

Eq. 5.5 provides an estimate of the forces acting on a water drop during sorting. For example, the maximum possible electrostatic force exerted on a drop in FC-40 is $F_{e l, \max }=$ $5.2 \mu N$ (given $R=30 \mu m, \epsilon_{r} \sim 2.1, d=3 \mu m, U=220 V_{R M S}$ ). This simple formula, however, does not take into account the $10 \mu \mathrm{m}$ gap between the electrodes. A numerical calculation including the gap shows a $20 \%$ reduction to $F_{e l, \max }=4.1 \mu \mathrm{N}$. This force calculated from the model is reasonably accurate. ${ }^{12,16}$ However, not taking into account the initial electric energy from the geometry without droplet gives an overestimation of the electric force of $\sim 17 \%$. In practice, more errors result from determination of the drop radius from experimental images, and the uncertainty in the dielectric thickness, which not only comprises of the thin PDMS layer, but also of a thin layer of dynamically entrapped oil which alters with different experimental parameters. ${ }^{17}$

In experiments aimed at finding the maximum sorting speeds for drops in FC-40 (Fig. 5 -5) we estimate $\Delta u$ to be $\sim 0.2 \mathrm{~m} / \mathrm{s}$, which, from Eq. 5.6, results in $F_{d r a g} \sim 1.2 \mu N$ (for $R=30 \mu \mathrm{m}, h=40 \mu \mathrm{m}, \mu=5 \mathrm{mPa} s$ ). Note that $\Delta u$ depends on $\alpha$ and that, given all the assumptions and simplifications made to arrive at Eq. 5.6, the values obtained for $F_{d}$ should be considered an order of magnitude estimation.

At the highest sorting speeds the condition of a constant velocity along the gap does not hold, and the drop will be pulled off the gap. For successful sorting, however, a drop only has to be deflected a small distance perpendicular to the flow direction to be sent into that particular outlet. Therefore, sorting can still be possible at slightly higher flowrates than our model predicts. Furthermore, even if $F_{e, \max } \geq F_{d} \sin (\alpha)$, at high sorting speeds two drops or a drop and the wall can come close together, resulting in hydrodynamic interactions that make our model less predictive.

\subsection{Results}

All experiments showed that a $100 \%$ reliable sorting (all drops sorted as intended) could be achieved by increasing the voltage above a flowrate dependent threshold. Different sorting modes were explored.

In one series of experiments, the sorter was conditioned such that, without electrical actuation, all drops would leave the sorter via the same outlet. This was achieved by 
applying an excess pressure to the other outlet. For several combinations of oil and water flow rates, it was examined how the efficiency of sorting into the hydraulically disfavored outlet depended on the applied voltage. The amplitude of this continuously applied AC signal was increased stepwise until the efficiency had reached $100 \%$. Per step, about 400 sorting events were recorded and analyzed with Matlab scripts. Besides counting the percentage of drops going into either outlet, also the injection frequency, size and velocity of the drops were measured. The results are summarized in Table 5-1 and Fig. 5-4.

The minimum voltage required for $100 \%$ sorting increases both with $Q_{\text {oil }}$ and with $Q_{\text {water }}$. This is in accordance with the expected dependence on the total flow rate: a larger $Q_{\text {total }}$ implies a stronger drag force on the drop, which in turn implies that a stronger electrostatic force is needed to overcome this bias. Fig. 5-4 shows that for each experiment at fixed flow rates, the sorting efficiency increases gradually with the voltage. Closer inspection of the movies revealed that sometimes, an ongoing accumulation of subsequent drops in the same outlet channel, led to a favoring of the other channel. This is ascribed to the increase in hydraulic resistance as an outlet fills with drops. Most important for practical applications, however, is that a consistent $100 \%$ sorting percentage can be obtained by choosing an excess voltage slightly over $V_{100 \%}$.

The size distribution of the drops is monodisperse, as is usual for flow focusing devices. Using the maximum diameter $2 R$ in a transmission image (e.g. Fig. 5-5) to characterize the drop size, we found the standard deviation to be $\sim 3 \%$. To simplify the comparison between the experiments at different flow rates, we aimed to keep $R$ constant. As Table 5 - 1 shows, an almost constant value of $32 \pm 2 \mu \mathrm{m}$ could be achieved. Because the channel height was only $40 \mu \mathrm{m}$, this implies that the drops are slightly flattened. The nearly constant $R$ was achieved by setting the oil flow rate $Q_{\text {oil }}$ much higher than the water flow rate $Q_{\text {water }}$. In this regime, the drop radius is mainly determined by the size of the orifice of the FFD. The drop volume, which shows a stronger dependence on $R$, is constant within $\sim 10 \%$ between experiments. Mass conservation then dictates that the drop generation frequency $f_{\text {drop }}$ (varied from 175 to $556 \mathrm{~s}^{-1}$ ) should be proportional to

Table 5-1 Electric sorting of water drops in FC40 oil, against a pressure bias, at different flow rates. From video analysis the average drop generation frequency $f_{\text {drop }}$, average drop velocity $v_{\text {drop }}$ and the average drop radius $R$ are determined. Also the inter-experimental standard deviation is determined. The last column shows the minimum voltage that is needed to change the destination of all drops from one outlet (in absence of voltage) to the other. The number is obtained from a linear fit to the data shown in Fig. 5-4. Higher flow rates require higher voltage to sort $100 \%$ of the drops.

\begin{tabular}{cccccc}
\hline \hline $\begin{array}{c}\text { Qoil } \\
(m L / h)\end{array}$ & $\begin{array}{c}\mathrm{Q}_{\text {water }} \\
(\mu L / h)\end{array}$ & $\begin{array}{c}f_{\text {drop }} \\
(H z)\end{array}$ & $\begin{array}{c}v_{\text {drop }} \\
(\mathrm{mm} / \mathrm{s})\end{array}$ & $\begin{array}{c}\mathrm{R} \\
(\mu \mathrm{m})\end{array}$ & $\begin{array}{c}V_{100 \% \text { sorted }} \\
(\mathrm{V})\end{array}$ \\
\hline 2.0 & 70 & $175 \pm 12$ & $81 \pm 6$ & $33.7 \pm 0.8$ & 103 \\
2.5 & 75 & $227 \pm 17$ & $110 \pm 7$ & $31.4 \pm 0.6$ & 111 \\
2.5 & 150 & $422 \pm 19$ & $113 \pm 8$ & $32.1 \pm 0.6$ & 128 \\
3.0 & 180 & $556 \pm 32$ & $141 \pm 6$ & $31.1 \pm 0.5$ & 139 \\
\hline \hline
\end{tabular}


$Q_{\text {water }}$ within comparable accuracy; this appears to be the case. The drop velocity was measured to be proportional to the total flow rate; also this is in agreement with expectations.

In another set of experiments, the sorter was used without the pressure bias. In this mode the sorter electrodes are always active, to ensure that all drops go into the 'waste' outlet. Switching the center electrode allows to select one or multiple drops to sort towards the other outlet. Compared to the previous sorting mode, the required electrostatic force (at given flow rates) is lower. Because only small biases (like slight asymmetries in the channel structure, or the mentioned effect of downstream accumulation, or misalignment of the sorting electrodes beneath the channel) need to be overcome, the drops require only a small deflection to be steered into the correct channel. This should allow for faster sorting. To explore the limits in sorting rate, we first continued the experiments in FC40 oil.

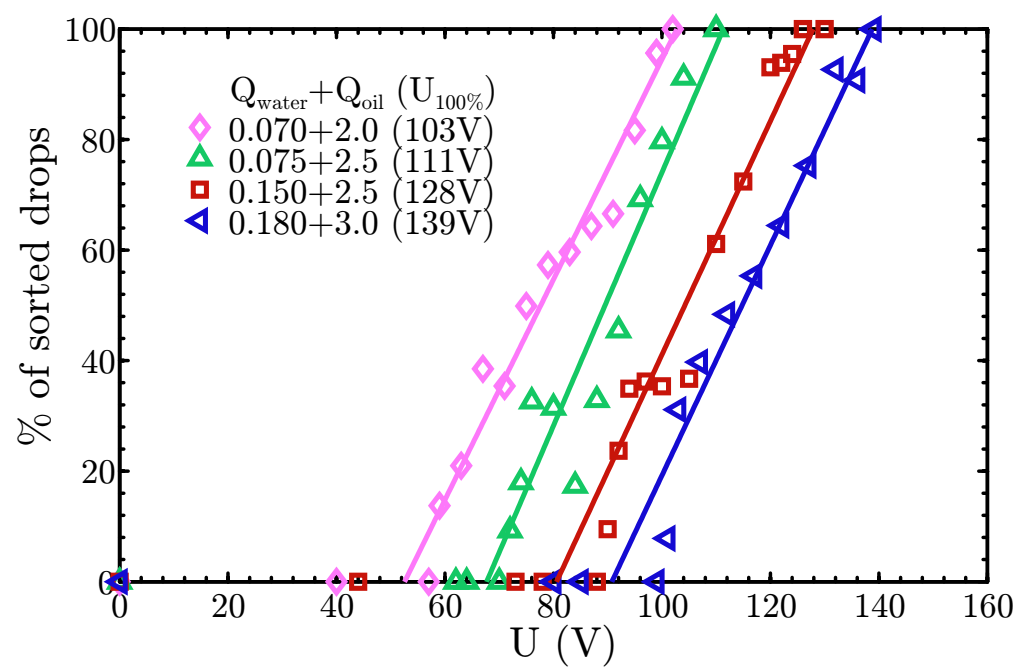

Fig. 5-4 Percentage of drops being actively sorted against the applied voltage for different water and (FC40) oil flow rate conditions in $\mathrm{mL} / \mathrm{h}$. Each data point is determined from a movie of $\sim 400$ drops.

Our protocol started with a maximization of the steering voltage; here we found that an upper limit for the voltage is not posed because of dielectric breakdown, but is posed by an electrical instability of the contact line resulting in the ejection of small satellite drops. ${ }^{18}$ Using a typical $50 \mathrm{~V} / \mu \mathrm{m}$ limit, dielectric breakdown is estimated to occur around 300 $V_{R M S}$ for the $3 \mu \mathrm{m}$ dielectric. Experiments show that contact line instability occurs around $250 V_{R M S}$. After fixing the voltage, pressure control on the oil and water phases was used to find the maximum rate of drop formation that would still result in reliable sorting. We consider sorting reliable if all drops are steered into the desired outlet. Preliminary experiments showed that this condition is more difficult to achieve than other sorting protocols, such as alternately steering drops to the outlets. 
We find that not the total flow rate, but the inter-drop distance ultimately limits the maximum sorting speed. At too high sorting rates drops stagnate at the tip of the Ychannel junction. The distance between the stagnant and consecutive drop then becomes too small, forcing the latter towards the undesired outlet via hydrodynamic interaction. The best achievement obtained for drops with $R \sim 25 \mu \mathrm{m}$ was a rate of $1200 \mathrm{~s}^{-1}$, which is a considerable improvement as compared to $25 \mathrm{~s}^{-1}$ in an earlier device. ${ }^{12}$ This experiment is shown in Fig. 5-5.
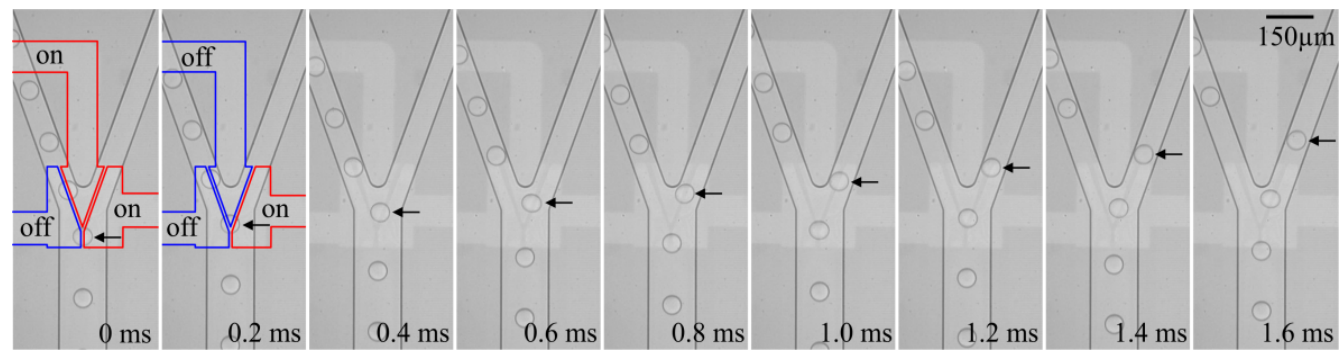

Fig. 5-5 shows a 1.6ms interval of the sorting of water drops in FC40 at 1200 per second. At $0 \mathrm{~ms}$ the center electrode is active, steering all drops to the left outlet. At 0.2ms the center electrode is switched off, resulting in drops going towards the right outlet. See Movie 5-1 for water drops sorted at 1200 drops per second.

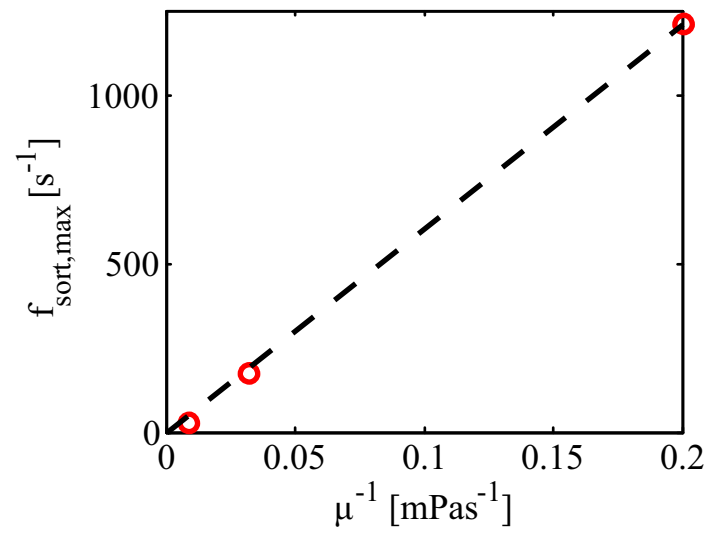

Fig. 5-6 The maximum sorting speeds achieved using oils of different viscosity $(\mu=169$, 32 and $5 \mathrm{mPas})$. In all experiments the channel geometry is the same, as well as the drop radius $(\sim 25 \mu \mathrm{m})$. The electrostatic force is independent of viscosity, while the drag force is proportional to the viscosity. Therefore the relation between maximum achievable sorting speed and reciprocal of the viscosity is close to linear. 
To inspect the role of the viscosity of the continuous phase, we also maximized the drop sorting rate for different oils. Although replacement of oil generally involves more changes than just the viscosity (e.g. also the interfacial tension), it was possible to make drops of a similar size $(R \sim 25 \mu \mathrm{m})$ and hence obtain the same (expected) relation between the voltage and the electric sorting force. Moreover, by adapting $Q_{\text {water }}$ to $Q_{\text {oil }}$ it was also possible to vary the overall flow rate while keeping the drop size constant. This allows quantitative comparison between the experiments and our model. According to the model, the maximum flow rate at which $100 \%$ sorting is still possible, should follow from a balance between the sorting force and the drag force. Keeping the maximum sorting force the same, it is then implied that the maximum sorting frequency should be inversely proportional to the oil viscosity. Experiments with 3 different oils suggests that this is indeed the case: sorting rates were $1213 \mathrm{~s}^{-1}$ for FC40 $(5 \mathrm{mPa} \mathrm{s}), 175 \mathrm{~s}^{-1}$ for light mineral oil $(31.5 \mathrm{mPa} \mathrm{s})$ and $30 \mathrm{~s}^{-1}$ for paraffin oil $(119 \mathrm{mPa} s)$. These viscosity values are obtained experimentally at an ambient temperature of $21^{\circ} \mathrm{C}$. Results are shown in Fig. $5-6$.

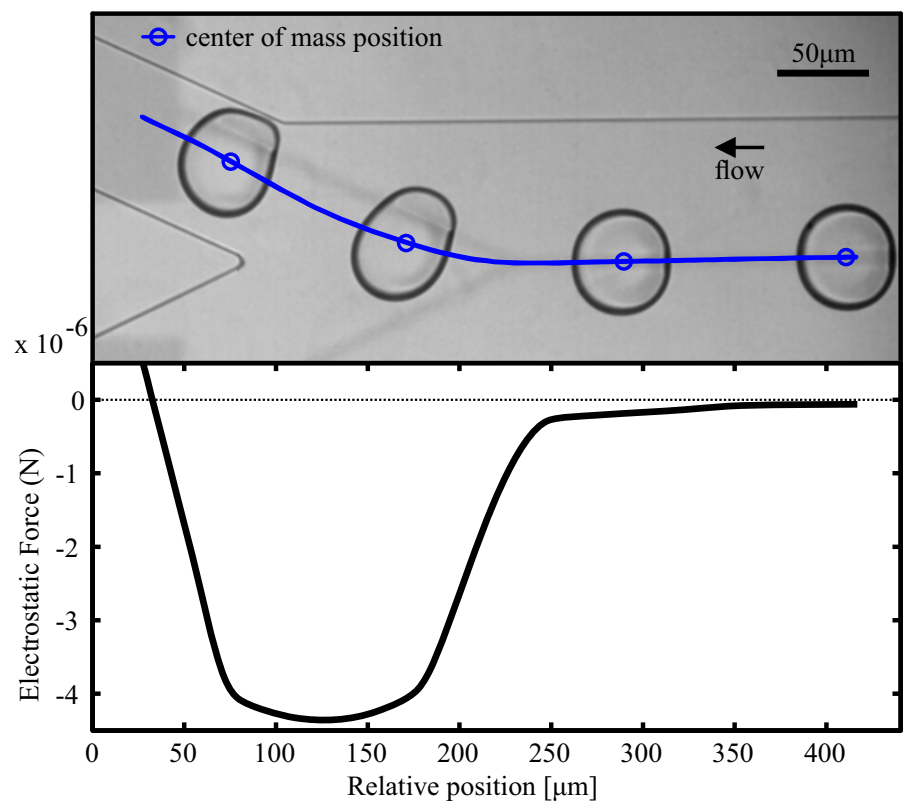

Fig. 5-7 (top) snapshot of four drops of $\mathrm{KCl}$ water dispersed in light mineral oil being sorted at 25 drops per second. Overlaid in blue: the averaged center of mass position of 27 consecutive drops undergoing sorting. (bottom) Graph of the averaged absolute electrostatic force on a series of 27 drops during sorting. See Movie 5-2 for the detection and measurement of drops during sorting and the resulting calculated electrostatic force. 
To further examine the sorting process under conditions optimized for maximum rate, we consider how the magnitude and direction of the sorting force develop along the path of the sorted drop. Fig. 5-7 shows a representative case where the sorting rate is (almost) maximal. For each video frame, the electrostatic force on the drops can be estimated. For this, we first measure the electrode areas covered by each individual drop. To translate this into a force, we use a numerically calculated calibration function for $F_{e}(x) / c R U^{2}$ that is similar to the expression of Eq. 5.4 and the graph in Fig. 5-3, but also takes into account the finite gap between the electrodes. Use of the relation between $x$, the distance of center of mass to the electrode gap, and $A_{1} / A_{2}$ for that case then allows to obtain the force from the measured areas. The underlying assumption that the drop footprint is circular is not met, because drops can deform under the influence of the electrostatic and drag force (as can be seen in Fig. 5-7). However, even an (estimated) error of 10-20\% still makes that the electrostatic force is known with much better accuracy than the hydrodynamic drag force as estimated via Eq. 5.6. While the electric and hydrodynamic forces are in the $\mu N$ range, the inertial force can simply be verified to be in the $p N$ range. This means that we can $i$ ) calculate the drag force by equating it to the electrostatic force, and $i$ i) compare it to the largest achievable electrostatic force. The comparison in Fig. 5-7 confirms that the electrostatic force exerted on the drop at the highest sorting rates is very close to the maximum value from our model. Conversely, a comparison with the drag force calculated via Eq. 5.6 suggests that the latter is underestimated by a factor of 4 by the model.

\subsection{Discussion}

It is interesting to examine which processes posed limitations to the sorting rates that could be achieved. The drop generation frequency was not a limiting factor in this work. The maximum voltage in our experiments was determined by the intrinsic instability of the drop against ejection of satellite drops, ${ }^{18}$ not by dielectric breakdown. Since this problem is caused by an excessive local electric field at the drop contact line, and the sorting force originates from the same field, this intrinsic limitation can only be improved by oil-water combinations with higher surface tension.

In our earliest channel designs, the tip of the Y-channel junction was very sharp, which led to drops being split at highest sorting rates. This problem was mitigated by giving the Y-junction a rounded corner (as in Fig. 5-5). However, this has the side effect of creating a wider stagnation zone at the junction of the outlet channels, where drops slow down as they near the channel wall. If the deflection of a drop is insufficient, this stagnation causes subsequent drops to approach each other too closely, leading either to coalescence or uncontrolled choice of the outlet channel by hydrodynamic interaction. This effect increases the required drop deflection for sorting, ultimately limiting the drop sorting frequency. To disfavor the close approach of the inner wall, the Y-shaped electrode geometry can be placed further away from Y-channel junction (see Fig. 5-7). Other biases, like an asymmetric presence of drops in either of the two outlets - causing a difference in hydraulic resistance - did not influence the sorting efficiency much in our experiments.

If the drag force becomes too large, the drops will no longer follow the electrode gap. In 
that case a smaller opening angle of the sorter would be needed, as this shifts the force balance in favor of the electric force. However this smaller angle would in turn require a longer distance between subsequent drops, since the active sorter should still be able to determine the fate of each individual drop. This issue can be remedied by adding two extra oil inlets just after the flow focusing junction, which increases the inter drop distance. This would, however, increase the drag force. In summary, it is not clear to what extent the handling speed of the present sorter could be further enhanced via geometric design.

Another aspect of practical relevance for any drop sorter is durability. While for each of the used oils the life time of the sorter was long enough to systematically explore the different voltages and flow rates, we did observe noteworthy differences in durability. Although each of the used oils shows similar wetting on PDMS (as was verified with experiments on PDMS slabs, where the contact angle of the water drop under the different oils is always larger than $160^{\circ}$ ), in a few cases we found that drops had begun to wet the dielectric substrate at the electrodes, ultimately leading to failure of the device. The time before this happens showed strong variations: drops of PBS (Phosphate Buffer Saline) solution in light mineral oil could be sorted at $100 \mathrm{~V}$ for at least 72 hours, without any noticeable detrimental effect on sorting, while drops of $\mathrm{KCl}$ solution in $\mathrm{FC}$-40 oil could be sorted at maximum rate at $220 \mathrm{~V}$ only for 6 hours. Additional diagnosis revealed that dielectric breakdown of the PDMS due to the applied voltage was not the cause of the degradation. The actual origin, however, could not be identified.

An explanation can be found by considering the oil film that separates the aqueous drop from the PDMS coating on the electrodes. If this film becomes too thin, direct contact of the drop can occur due to Van der Waals attraction, even though the Hamaker constants are small $\left(<2 \times 10^{21} \mathrm{~J}\right)$. The breakdown probability of the lubrication layer depends both on the film thickness and the 'exposure time' of the drop to the electric field, i.e. the time needed for the drop to pass the electrode. Here the electric field drives the thinning of the layer of oil, while its viscosity influences the time scale of this process. The thickness $h$ of a film subjected to an electric field is known to scale with the applied voltage as ${ }^{17}$

$$
h \sim(d \sqrt{R})^{2 / 3}\left(\frac{C a}{\eta}\right)^{2 / 3},
$$

where $C a$ is the capillary number

$$
C a=\frac{\mu v}{\gamma},
$$

with $\mu$ the oil viscosity and $\gamma$ the oil/water interfacial tension, and $\eta$ the electrowetting number

$$
\eta=\frac{\epsilon_{0} \epsilon_{r} U^{2}}{2 d \gamma}
$$

This suggests that, for a given drop radius $R$ and insulator thickness $d$, the film thickness $h \propto C a^{2 / 3} U^{-4 / 3}$. Therefore, three factors can promote the wetting of the substrate by 
water: a lower oil viscosity, a lower drop velocity, and a higher voltage. It is worthwhile to note that the capillary numbers for the maximum sorting speed experiments with different oils are the same, because $\gamma$ remains $\sim 50 \mathrm{mN} / \mathrm{m}$, and $\mu v$ is proportional to the drag force. Comparison between the before mentioned wetting case of FC-40 and the nonwetting mineral oil reveals that the FC-40 case indeed has a (sixteen times) thinner oil film.

Another aspect of durability is that also the content of the drop can favor wetting of the surface. In particular, amphiphilic biological molecules like proteins have a tendency to adsorb at the oil/water interface and subsequently at the channel walls, thereby making them hydrophilic. In our case this was observed with Dulbecco's Modified Eagle Medium as the drop phase. However, this type of issue is not an inherent aspect of our sorter. Mitigations of this aspect are already known, e.g. by using specific surfactants to cover the interface of the drop, ${ }^{19}$ or the channel walls. ${ }^{20}$

\subsection{Conclusion}

The present study demonstrates that simple design rules can be used to enhance the rate of drop sorting, when compared to an earlier prototype electrostatic sorter. The electrostatic force exerted by a coplanar electrode geometry can be controlled by either the geometry of the design, e.g. insulator thickness, electrode size, gap width, or the applied voltage. An additional way to favor the balance between the steering electrostatic and the opposing hydrodynamic forces, is to reduce the latter. Use of a low viscosity oil, and a small opening angle of the sorter were both applied, and contributed to the achievement of sorting rates up to $1200 \mathrm{~s}^{-1}$ for $160 \mathrm{pL}$ drops. The use of smaller drops could be yet another way to increase the sorting rate, since this would lower the drag force more strongly than the steering force. Ultimately, the specific application will determine to what extent the various degrees of freedom can be used: for example cells in drops might require specific drop volumes and (bio) compatible oils. We remark here that electrowetting sorters do not pose limitations to the drop sample material via electric fields: the sorting principle still works at physiological high salt concentrations, and the contents of the drop are not exposed to electric fields. The present work also showed that, although the proportionality of the drag force with drop velocity and oil viscosity provides a global design rule, an analytical estimate of this force is difficult to obtain, even if the presence of other nearby drops can be neglected.

Finally, we like to point out that from a practical point of view our sorter has the following advantages: $i$ ) the fabrication costs are low, due to the use of standard PDMS and ITO electrodes, ii) 100\% reliability of sorting can be achieved for a wide range of flow rates, iii) the durability of the device is promising, evidenced by a sorting experiment that lasted 3 days, iv) the sorting forces are strong, approximately 1000 times larger than reported in DEP sorting ${ }^{6}$ at a 10 times lower voltage, v) sorting speeds exceeding $1 \mathrm{kHz}$ can be reached, vi) sorting of highly conductive drops can be achieved, vii) the drop contents are not exposed to electric currents or Joule heating (as in DEP), viii) the physical concepts are simple, which allows users to design their own electrode geometries for their specific application. 


\subsection{Future Prospects}

In preliminary sorting experiments of drops containing fluorescently labeled cells, we were able to separate cell-laden drops from empty ones by sorting at 400 drops per second. The use of a surfactant served to protect all interfaces from the adsorption of drop contents. The decreased oil-water surface tension did not negatively affect the sorting. Sensitive detection of the cells fluorescence using a Photo Multiplier Tube was used in combination with the speed of a Field Programmable Gate Array to trigger the amplifier of the steering signal at a rate that was so fast, that the focal spot for the fluorescent excitation actually had to be placed just micrometers in front of the Y-junction. For a recording of this experiment, which underlines the potential of our method, see Movie 5-3 for live sorting of cell containing drops at 400 drops per second.

\section{Acknowledgements}

We like to thank Dr. Aigars Piruska and Prof. Wilhelm T.S. Huck from the PhysicalOrganic Chemistry group at the Radboud University (Netherlands) for performing the cell sorting experiments on their high speed fluorescence detection and actuation setup, as well as supplying fluorescently labeled cells and fluorinated surfactants.

\section{References}

1. Theberge, A.B., F. Courtois, Y. Schaerli, et al., Microdroplets in microfluidics: an evolving platform for discoveries in chemistry and biology. Angew Chem Int Ed Engl, 2010. 49(34): p. 5846-68.

2. Theberge, A.B., E. Mayot, A. El Harrak, et al., Microfluidic platform for combinatorial synthesis in picolitre droplets. Lab Chip, 2012. 12(7): p. 1320-6.

3. Abate, A.R., C.H. Chen, J.J. Agresti, et al., Beating Poisson encapsulation statistics using close-packed ordering. Lab on a Chip, 2009. 9(18): p. 2628-31.

4. Kemna, E.W., R.M. Schoeman, F. Wolbers, et al., High-yield cell ordering and deterministic cell-in-droplet encapsulation using Dean flow in a curved microchannel. Lab Chip, 2012. 12(16): p. 2881-7.

5. Baret, J.C., O.J. Miller, V. Taly, et al., Fluorescence-activated droplet sorting (FADS): efficient microfluidic cell sorting based on enzymatic activity. Lab on a Chip, 2009. 9(13): p. $1850-8$.

6. Ahn, K., C. Kerbage, T.P. Hunt, et al., Dielectrophoretic manipulation of drops for high-speed microfluidic sorting devices. Applied Physics Letters, 2006. 88(2): p. 3.

7. Franke, T., A.R. Abate, D.A. Weitz, et al., Surface acoustic wave (SAW) directed droplet flow in microfluidics for PDMS devices. Lab Chip, 2009. 9(18): p. 2625-7.

8. Wu, L., P. Chen, Y. Dong, et al., Encapsulation of single cells on a microfluidic device integrating droplet generation with fluorescence-activated droplet sorting. Biomed Microdevices, 2013. 15(3): p. 553-60. 
9. Ahn, B., K. Lee, R. Panchapakesan, et al., On-demand electrostatic droplet charging and sorting. Biomicrofluidics, 2011. 5(2): p. 24113.

10. Fradet, E., C. McDougall, P. Abbyad, et al., Combining rails and anchors with laser forcing for selective manipulation within 2D droplet arrays. Lab on a Chip, 2011. 11(24): p. 4228-34.

11. Abate, A.R., J.J. Agresti, and D.A. Weitz, Microfluidic sorting with high-speed single-layer membrane valves. Applied Physics Letters, 2010. 96(20).

12. de Ruiter, R., A.M. Pit, V.M. de Oliveira, et al., Electrostatic potential wells for on-demand drop manipulation in microchannels. Lab Chip, 2014. 14(5): p. 883-91.

13. Duffy, D.C., J.C. McDonald, O.J. Schueller, et al., Rapid Prototyping of Microfluidic Systems in Poly(dimethylsiloxane). Anal Chem, 1998. 70(23): p. 4974-84.

14. 't Mannetje, D., S. Ghosh, R. Lagraauw, et al., Trapping of drops by wetting defects. Nat Commun, 2014. 5: p. 3559.

15. Mugele, F. and J.-C. Baret, Electrowetting: from basics to applications. Journal of Physics: Condensed Matter, 2005. 17(28): p. R705.

16. 't Mannetje, D., A. Banpurkar, H. Koppelman, et al., Electrically tunable wetting defects characterized by a simple capillary force sensor. Langmuir, 2013. 29(31): p. 9944-9.

17. Staicu, A. and F. Mugele, Electrowetting-induced oil film entrapment and instability. Phys Rev Lett, 2006. 97(16): p. 167801.

18. Mugele, F. and S. Herminghaus, Electrostatic stabilization of fluid microstructures. Applied Physics Letters, 2002. 81(12): p. 2303-5.

19. Baret, J.C., Surfactants in droplet-based microfluidics. Lab on a Chip, 2012. 12(3): p. 422-33.

20. Zhou, J.W., D.A. Khodakov, A.V. Ellis, et al., Surface modification for PDMS-based microfluidic devices. Electrophoresis, 2012. 33(1): p. 89-104. 



\section{6}

\section{Electrowetting in the dielectric regime}

Electrowetting (EW) is a phenomenon that describes the decrease of the contact angle of drops of partially wetting conductive liquids upon applying a voltage between the liquid and an electrode on the substrate that is usually covered by a thin hydrophobic dielectric coating. In many applications including lab-on-a-chip systems, however, the electrostatic forces that can be exerted by sequential actuation of patterned electrodes to displace drops are more important than the reduction of the contact angle. Such forces are known as dielectrophoretic driving forces not only for conductive but also for dielectric liquids. Here, we investigate the relative importance of electric conductivity and dielectric permittivity for droplet actuation and we analyze the role of geometrical parameters such as drop size, insulator thickness and electrode spacing for sessile and sandwiched drops near substrates with embedded electrodes. Electric energies and resulting forces are calculated numerically for a wide range of parameters using a finite element solver. For simple cases, approximate lumped parameter models are developed to represent the behavior of the drop. Our analysis highlights the relative importance of translational and internal (deformational) degrees of freedom for sessile and for sandwiched drops. At first glance surprisingly, we find that the electric forces to actuate sandwiched drops in EW-based microfluidic sorting devices remain strong at high frequencies, while in general the EW effect strongly decreases for liquids in this so-called dielectric regime.

This chapter is in preparation for submission:

A.M. Pit, A. Cavalli, D. van den Ende, M.H.G. Duits and F. Mugele, "Electrowetting in the dielectric regime", (2017) 


\subsection{Introduction}

Droplet-based microfluidics has become a popular tool in lab-on-a-chip technology. Besides the general advantages of miniaturization, parallelization and high speed, the specific aspect of compartmentalization offers unique possibilities like the screening of many individual samples, and the use of statistical analysis. ${ }^{1,2}$ A key requirement for application of the technology is the ability to send each individual droplet to its designated location, with high reliability. Both passive and active methods have been implemented ${ }^{3}$. Passive methods include the use of surface wettability gradients, ${ }^{4}$ or geometrical structures. ${ }^{5-7}$ Active methods can be pneumatic membrane valves, surface acoustic waves, magnetic or optical tweezers, dielectrophoresis (DEP) and electrowetting-on-dielectric (EWOD). See Chapter 2 for more details.

One of the most successful techniques for active droplet manipulations in applications is digital microfluidics (DMF), where EWOD is used to actuate droplets. The main advantages are the easy integration with existing electronic systems, and the relatively large forces that can be exerted, yielding a high level of control over the droplets. This has resulted in many different forms of manipulations, like guiding, transport, mixing, jumping, splitting and merging of droplets. ${ }^{3,8}$ Especially in bioapplications DMF has found fruitful use for instance in combination with SPRi, PCR, MALDI-MS and more. ${ }^{9}$

The term electrowetting $(\mathrm{EW})^{10}$ refers to the decrease in contact angle (an improved wetting) that is observed when a droplet is subjected to an electric field. This contact angle change is merely an observable consequence of the ability of the drop to optimize its free energy after the electrically induced change in the energy landscape. This shifted minimum between electric and surface energy is described by the Young-Lippmann equation. However, in microfluidic devices the consequences of EW are different than for macroscopic sessile drops. Contact angles are not easily observable. Also, the confinement of the liquid by the walls decreases the freedom of deformation making capillary forces of less interest. Hence, it makes sense to analyze the (center of mass) displacement of the droplet instead. This can be achieved by considering the electric energy (or force) as a function of droplet position with respect to the electrode(s).

Both EWOD scenarios (contact angle change and droplet displacement) can be captured by considering electrostatics. While the physical ingredients of EWOD are well-known, its applications can sometimes present outcomes that are surprising. This can happen in particular if the used liquid phase differs from the standard aqueous systems, if the AC frequency becomes high, or if the relative dimensions of droplets, insulators and actuating electrodes become atypical.

In this chapter we focus on the situation that appears when using electrowetting in microfluidic devices. In this case the length scales of the insulator and the droplet approach each other, which, as we will show, changes the electrowetting behavior when using $\mathrm{AC}$ frequencies beyond which the liquid can no longer be considered a conductor. 


\subsection{Background}

\subsubsection{Frequency dependent contact angle}

The relationship between electrowetting and the applied frequency has already been researched in many publications. In particular papers by T.B. Jones et al. give a clear analytical picture of how the electric forces depend on the applied frequency. ${ }^{11-13}$ They show that (capacitive) electric circuit modeling and closed surface integration of the Maxwell stress both allow the calculation of the electric forces, and importantly, that these forces - despite acting at the contact line - do not depend on the contact angle of the droplet. This may seem counterintuitive, since the contact angle changes while electrowetting, but is verified by their energy and pressure arguments. For the parallel plate geometry, where a droplet is sandwiched between parallel electrodes as shown in Fig. 6-1b, they also determine a critical transition frequency $f_{c}$, where the liquid transitions from a conductor to a dielectric as:

$$
f_{c}=\frac{\sigma_{l}}{2 \pi \epsilon_{0}\left(\epsilon_{l}+\epsilon_{d} D / 2 d\right)}
$$

with $\sigma_{l}$ the liquid conductivity, $\epsilon_{0}$ the permittivity of vacuum, $\epsilon_{l}$ the relative permittivity of the liquid, $\epsilon_{d}$ the relative permittivity of the insulator, $D$ the liquid distance between the plates and $d$ the thickness of the dielectric insulator. For a numerical investigation of $\mathrm{AC}$ electrowetting we can look at the works of K.H. Kang et al. ${ }^{14}$, which for instance enabled the calculation of electrohydrodynamic flow inside the droplet undergoing electrowetting..$^{15,16}$

One general observation shared by all aforementioned papers is that the electrowetting effect severely decreases when using high frequencies, i.e. frequencies above the critical frequency where the liquid can no longer be considered a conductor. ${ }^{13,14}$ This decrease is observed for different electrowetting geometries. As an example we can use the classical EWOD geometry. In this geometry a sessile droplet atop a thin insulator decreases its contact angle upon application of an electric potential difference between the wire in the droplet and the insulated electrode beneath it (Fig. 6-1a). It has been observed that for this geometry electrowetting only works for conductive droplets, while at higher frequencies there is almost no contact angle decrease (Fig. 6-1c). ${ }^{14}$

The parallel plate electrode geometry (See Fig. 6-1b) is often used in the so-called Digital Microfluidics (DMF) setup, which is capable of moving droplets over many electrodes. For this geometry Chatterjee et al. test for a large range of polar liquids whether movement of the droplet occurs or not, for frequencies of 0.01, 0.1, 1 and $8 \mathrm{kHz}$, and for spacing between the electrodes from 100 to $300 \mu m .{ }^{17}$ They found that some liquids can be actuated for all combinations of these variables, and some for none. Interestingly, they also find that some liquids become unresponsive at high frequency, and some only for the smallest spacing. Also, experiments in our lab have confirmed that for the parallel plate geometry at high frequencies drops of different conductivity show virtually no contact angle change upon increasing the voltage frequency. ${ }^{18}$ In this geometry the change in contact angle $\Delta \cos \theta$ scales with the electric energy for low voltages. (See Fig. 6-1d) 
(a)
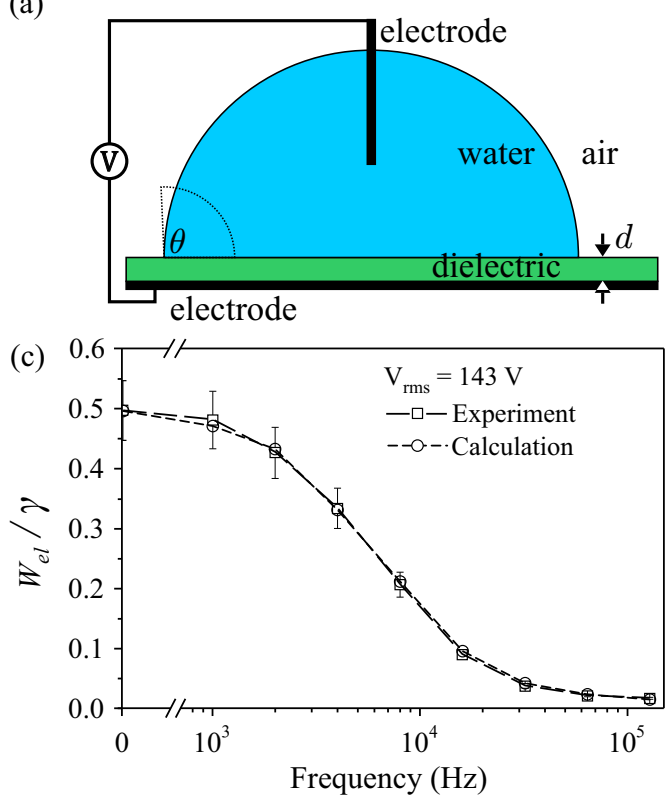

(b)
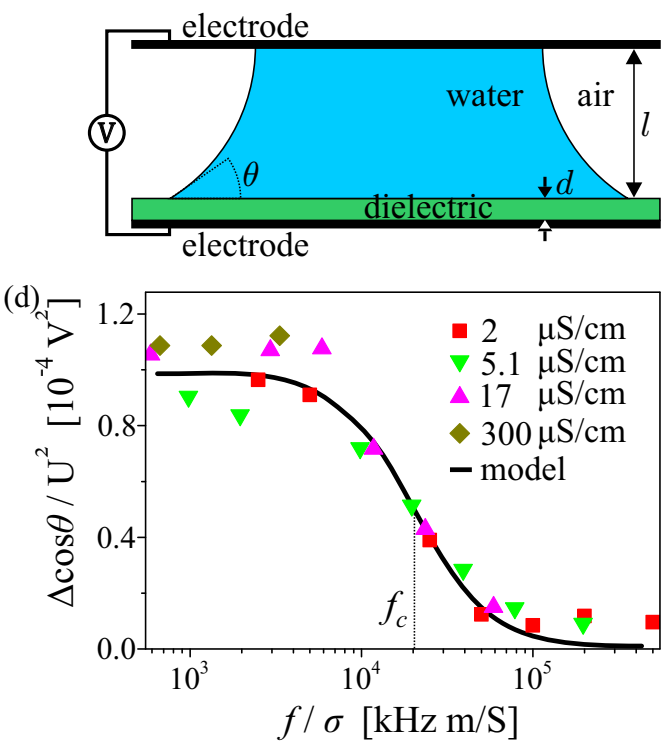

Fig. 6-1 Schematic overviews of: (a) the classical EWOD setup, where a metal wire is inserted into a macroscopic droplet, and (b) the parallel plate EWOD setup. (c) The frequency dependence of the electric energy (divided by the interfacial tension $\gamma$ ) for the classical EWOD setup. Reproduced with permission of Springer from Microfluid Nanofluid (2008) 5:263-271.14 (d) The frequency dependence (normalized with the conductivity $\sigma$ ) of the measured change in contact angle for the parallel plate EWOD geometry. ${ }^{18}$ For both geometries holds that beyond a critical frequency $f_{c}$ the liquid may be considered a dielectric and there is virtually no EW as observed by the small change in contact angle.

\subsubsection{Sorting efficiency for conductive and dielectric droplets}

Next let us consider the use of co-planar electrode geometry to sort tiny (i.e. $100 \mathrm{pL}$ sized) droplets in a microfluidic chip. In previous studies (Chapter 3 and 5) we have shown that conductive water droplets immersed in oil could be sorted effectively by directing them towards one of two outlets of a Y-junction via the electric force. ${ }^{19}$ In the present study we extend the sorting experiments by varying the AC frequency (Section 6.4), increasing it to the point that the droplets can no longer be considered perfect conductors. Based on the graphs in Fig. 6-1, the expectation is that the sorting efficiency should reduce severely, since the force that causes the contact angle change and the sorting efficiency have the same electric origin. In section 6.4 we will show, however, that within experimental errors the efficiency of droplet sorting in the microchannel does not decrease with frequency. 


\subsection{Scope of this chapter}

The aforementioned observations can be captured by considering the electric forces that act on the contactline. In these terms, it is remarkable that classical contact angle experiments on millimeter scale sessile droplets show a severe reduction in electrowetting force when going to high $\mathrm{AC}$ frequency, while in microfluidic sorting experiments this force is hardly reduced. In this chapter we analyze these seemingly contradictory experimental observations in more detail. We remind ourselves that for contact angle measurements the EWOD behavior can be understood from the energy minimization of the (adjustable) electric energy and the (conservative) surface energies. For the microfluidic sorting experiments the droplet is confined between walls, and only limited deformation is possible. Therefore, surface tension plays a less important role. At the same time the contact angle is not easily measured inside the channel and is thus impractical to use to describe the electric force. Given these contemplations and the notion that our experimental observations at high frequencies are most likely not caused by a frequency dependence of the surface tension, we explain the electrowetting behavior by focusing entirely on the electric energy directly, ignoring the changes in surface energy.

Lumped (electric) element models (LEM) have often been used to explain the phenomenon of electrowetting, including the transition from conductive to dielectric regime. Also, electrowetting of various types of dielectric liquids has been researched extensively. Remarkably, to our knowledge, no one has reported how the efficiency of EW can be maintained even deep in the dielectric regime, for appropriate combinations of length scales of the liquid and insulator, even though the existing theories show exactly what is required. The critical AC frequency $f_{c}$ plays an important role in this analysis. The conductivity and permittivity of the fluid phase, and geometrical factors involving both the droplet and the substrate are important in classifying, which regime (conductive or dielectric) applies in a particular experiment. This should be of interest for the manipulation of fluids for which the conductivity is so low, that they are dielectric even at the usual $\mathrm{AC}$ frequencies used in electrowetting. As we will see, the optimization of dimensions means that electric forces can be optimized, albeit to achieve a sorting force or a contact angle reduction.

As described by Jones $(2002)^{11}$ for simple geometries - like a liquid sandwiched between parallel plate electrodes - a lumped element model (LEM) gives analytical solutions of the electric potential field. The LEM allows calculation of the electric energy gain involved in electrowetting, and thereby an understanding of the driving force of the wetting of liquids that are conductive, dielectric or in-between. We will derive the expressions for this model, and examine its outcomes to find explicit expressions that dictate the difference between the electric force in the conductive and dielectric regime, which in turn allow the optimization of the electric force in the dielectric regime.

Droplets atop a co-planar electrode geometry - as is the case in the microfluidic channel - present a more complex case, particularly if the liquid is non-conducting. In that case the liquid cannot be represented as a parallel plate capacitor, and electric fringe fields around the electrode edges (and droplet contact line) cause sharp gradients in the electric field, which can play a role. To analyze this case, we use a simplified version of the numerical model based on the work of Hong, et al. $(2008)^{14}$ in which we combine the 
Poisson equation for electrostatics with the charge continuity equation to calculate the electric potential field. The results are then used to calculate the electric energy at each droplet position, and from that the electric force acting on the droplet. As we will see, in certain cases these forces are comparable for conductive and non-conductive drops, thereby explaining our observations.

\subsection{Sorting efficiency experiment}

Given that literature and our own experiments showed that the electrowetting force is strongly diminished in the dielectric regime (Fig. 6-1), we initially expected our EWOD based microfluidic sorter - described in Chapter 5 - to be able to distinguish between conductive and dielectric droplets simply by going to higher $\mathrm{AC}$ frequencies. Initial experiments however showed no clear difference in sorting force between DI water and water made extra conductive by adding $\mathrm{KCl}(3 \mathrm{mS} / \mathrm{cm})$. The following experiment was devised to measure the efficiency of sorting against frequency.

\subsubsection{Method}

The microfluidic sorter consists of three electrodes, placed just below the channel, and separated horizontally by a $10 \mu \mathrm{m}$ Y-shaped gap. In this co-planar EWOD geometry (as highlighted in Fig. 6-2a), an electric centering force is generated orthogonal to the electrode gap. ${ }^{20}$ The thereby induced motion of the droplet is counteracted by a hydrodynamic force, which puts a penalty on deviation from flow lines. The Y-junction in the PDMS channel assists in determining the fate of the drop; it forces the drop to go either left or right. Hence a small electrically induced deflection is already sufficient to seal the fate of the drop upon arrival at the junction.
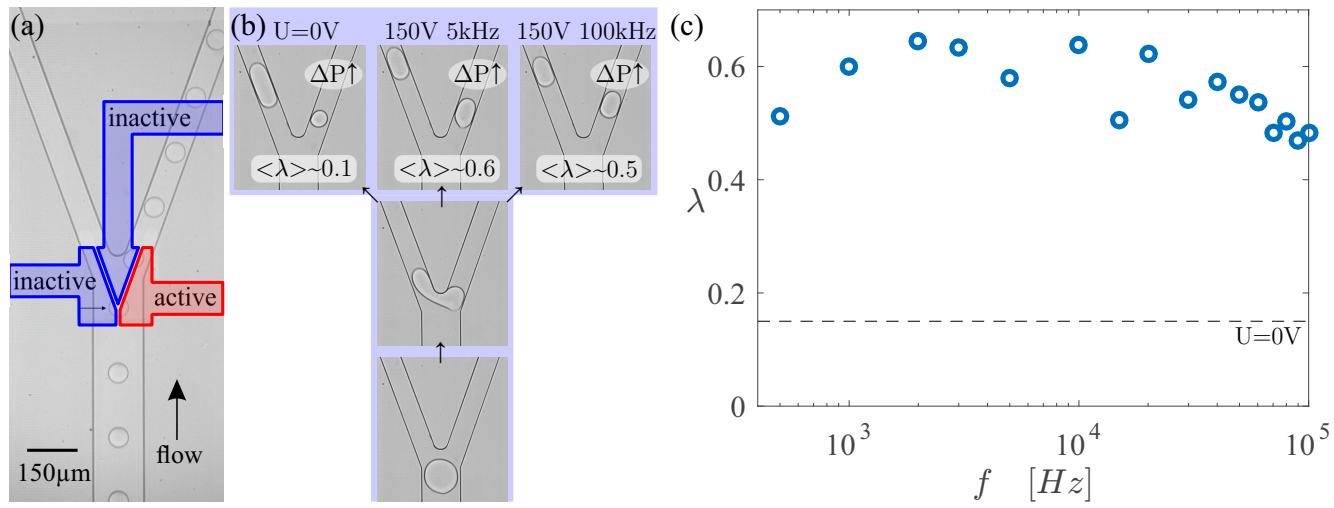

Fig. 6-2 (a) Top view of the sorter indicating the position of the electrodes (blue: ground, red: active), (b) Top view of large DI water drops splitting at the sorting junction under the influence of a pressure bias and zero (left), $5 \mathrm{kHz}$ (middle) and 100kHz (right) applied potential. (c) The frequency dependence of the sorting efficiency. There is hardly any difference between sorting in the conductive (《18 $\mathrm{kHz}$ ) or dielectric regime (》18 $\mathrm{kHz}$ ). 
To examine the influence of the $\mathrm{AC}$ frequency on the sorting force, the sorting experiment had to be redesigned. We define the sorting efficiency $(\lambda)$ as the fraction of the droplet phase that ends up in the targeted outlet. In previous experiments with small droplets (radius $R \sim 50 \mu \mathrm{m}$ ) we observed that given a certain balance of electric and hydrodynamic forces nearly all droplets choose the same outlet. This steep transition from one outlet to the other does not resemble an actual sorting efficiency, but a sorting cutoff position. To get a better correlation between the changing electrostatic force and the measured sorting efficiency we chose to use larger droplets which break up into two fragments at the Y-junction (see Fig. 6-2b) and measure the volume fractions in each channel. This generates a more gradual dependence of $\lambda$ on the electric force experienced by the droplets. To increase the detection range for $\lambda$, we apply a pressure bias between the microchannel outlets, such that without applying a voltage only $15 \%$ of the volume of DI water droplets end up in the channel for active sorting $(\lambda \sim 0.15)$. Next, a constant potential of $150 \mathrm{~V}$ is applied such that $\sim 60 \%$ of the droplets are actively sorted in the conductive regime $(\lambda \sim 0.6)$. Maintaining the (RMS) amplitude of the potential, $\lambda$ is then measured at various frequencies from 0.5 to $150 \mathrm{kHz}$. A decrease in electric force should result in a lower value for the efficiency.

\subsubsection{Result}

The experimental results in Fig. 6-2c show that DI water droplets are sorted at a fairly constant efficiency, even at frequencies as high as $100 \mathrm{kHz}$. Even above $100 \mathrm{kHz}$ the sorting efficiency decreases only slightly (not shown), which was caused by the limitations of the High Voltage amplifier (TREK PZD700A). Hence for the microfluidic sorter the efficiency is stable at least up to $100 \mathrm{kHz}$. In contrast, in classical EW with mm-sized droplets, the critical frequency $f_{c}$ where the droplet becomes dielectric, lies around $4 \mathrm{kHz}$. Filling in the values for the microfluidic experiment in Eq. 6.1 we find an approximate $f_{c}$ of $18 \mathrm{kHz}$. This experiment indicates that the sorter can sort dielectric droplets.

To further examine the sorting efficiency, the DI water is replaced with propylene carbonate (PC) which is approximately 10x less conductive (and thus $f_{c}$ lies 10x lower), yet still has a high relative permittivity $(\epsilon \sim 65)$. As shown in Movie 6-1, at $50 \mathrm{kHz}$, thus well above the critical frequency of PC, the sorting efficiency is still high. This proves that sorting also works for dielectric drops.

\subsection{Analytical model}

In order to explain the seemingly contradictory observations of electrowetting in the dielectric regime for the classical EWOD and microfluidic case we need to go back to the basics of electrostatics. The simplest geometry to start with is the parallel plate geometry. For this geometry, as depicted in Fig. 6-3, the Lumped Element Model (LEM) as described by Jones, et al. (2003) ${ }^{12}$ can determine the electric energy accurately. The parallel plate geometry is also the simplest geometry that can be analyzed by a 1-dimensional version of the numerical model described in Section 6.6. 


\subsubsection{Lumped Element Model}

In the case of electrowetting, the electric potential energy $W_{e}$ can be equated to the energy stored in the electric fields $\boldsymbol{E}$. The electric fields are present in the dielectric materials between electrodes of different potential, very similar to capacitors in an electronic device. A simple way to capture these properties is a Lumped Element Model (LEM). ${ }^{12}$ In this model, sketched in Fig. 6-3, the individual, parallel layers (electrodes, insulators, and liquids) can be described by parallel plate capacitors in series. For each parallel plate capacitor the electric field can be considered constant (ignoring edge effects), it is orthogonal to the plate, and it is given as $E_{z, i}=U_{i} / h_{i}$, with $U_{i}$ the potential falling over a capacitor and $h_{i}$ the distance between the parallel interfaces. In Fig. 6-3 the thin insulating layers covering the electrodes (dielectric constant $\epsilon_{d}$, thickness $h_{d}$ ) and the nonpolar ambient medium, e.g. air or oil, (dielectric constant $\sim \epsilon_{d}$, thickness $h_{l}$ ) are represented as parallel plate capacitors. The polarizable liquid (dielectric constant $\epsilon_{l} \gg$ $\epsilon_{d}$, conductivity $\sigma_{l}$, thickness $h_{l}$ ) is represented as a resistor and capacitor in parallel. Effectively, the LEM consists of 2 parallel circuits. Part I the insulators in series with the liquid. Part II the insulators in series with the ambient medium.

Since the ambient fluid can be replaced by the conductive liquid, the total energy will depend on the configuration, i.e. the position of the liquid-liquid interface. Using this LEM, we can get a quick overview of how different geometric parameters can influence the electric energy stored in the electric fields. In the diagram sketched in Fig. 6-3, the total electric energy is the sum of the energies of each capacitor in our system minus the energy expended by the battery in charging the active electrode:

$$
W_{E}=\sum W_{e, i}-W_{e, b a t}
$$

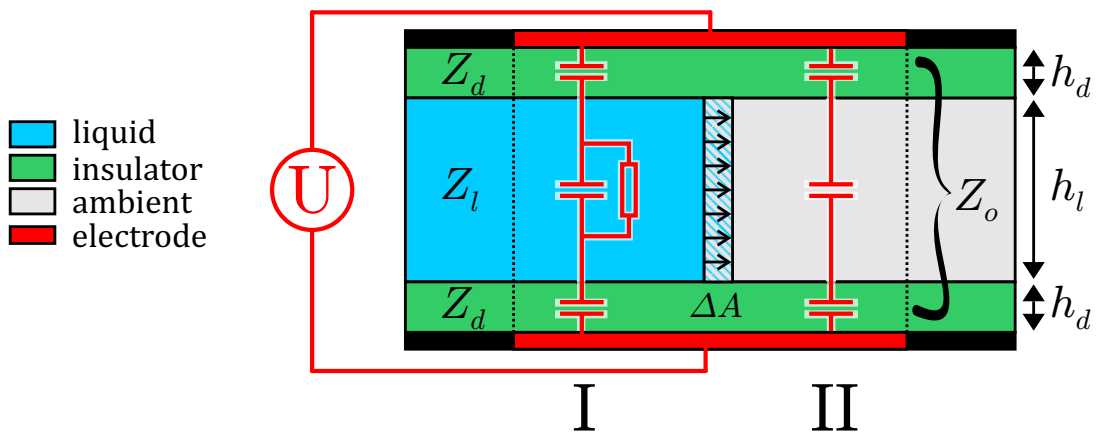

Fig. 6-3 Schematic of a parallel plate EWOD geometry and the electric circuit representations for the Lumped Element Model. On the left (I) the conductive polarizable liquid is represented by a resistor and capacitor in parallel. On the right (II) the dielectric ambient medium is simply a capacitor. When the polar liquid displaces a certain volume $\left(h_{l} \Delta A\right)$ of the ambient fluid this will result in an increase in electric energy. 
From electrostatics we know that the energy stored in each capacitor is

$$
W_{e}=\frac{1}{2} c A U^{2}
$$

with $c$ the capacitance per unit area $A$, and $U$ the potential difference across each capacitor. For now we only consider the left side (I) of Fig. 6-3. In part I, the impedances $\boldsymbol{Z}_{\boldsymbol{d}}, \boldsymbol{Z}_{\boldsymbol{l}}$ and $\boldsymbol{Z}_{\boldsymbol{d}}$ are in series and thus the total applied voltage $U_{T}$ is divided to partly fall across each insulator $\left(\boldsymbol{U}_{\boldsymbol{d}}\right)$, and the remaining part falls across the liquid $\left(\boldsymbol{U}_{\boldsymbol{l}}\right)$. In the Appendix 6A2 we prove that for our system the energy stored in the capacitors is half of the energy supplied by the battery. Therefore Eq. 6.2 and 6.3 can be rewritten as:

$$
W_{E}=-\frac{1}{2}\left(2 C_{\mathrm{d}} \boldsymbol{U}_{\mathbf{d}}^{\mathbf{2}}+C_{\mathrm{l}} \boldsymbol{U}_{\mathbf{l}}^{\mathbf{2}}\right)=-\frac{1}{2} U_{T}^{2}\left(2 C_{\mathrm{d}} \frac{\boldsymbol{U}_{\mathbf{d}}^{\mathbf{2}}}{\mathrm{U}_{\mathrm{T}}^{2}}+C_{1} \frac{\boldsymbol{U}_{\mathbf{l}}^{\mathbf{2}}}{\mathrm{U}_{\mathrm{T}}^{2}}\right)
$$

Since the impedances are in series, the current through them is the same. This means that the voltage division ratios $\left(\boldsymbol{U}_{\boldsymbol{i}} / U_{T}\right)$ are equal to the impedance ratio of the insulators $\left(\boldsymbol{Z}_{\boldsymbol{d}} / \boldsymbol{Z}_{\boldsymbol{T}}\right)$ and liquid $\left(\boldsymbol{Z}_{\boldsymbol{l}} / \boldsymbol{Z}_{\boldsymbol{T}}\right)$, with $\boldsymbol{Z}_{\boldsymbol{T}}=2 \boldsymbol{Z}_{\boldsymbol{d}}+\boldsymbol{Z}_{\boldsymbol{l}}$ the total impedance. Given the arrangement of the capacitors and resistor, the frequency dependence of the impedance ratios is given as (See Appendix 6A2):

$$
\left|\frac{\boldsymbol{Z}_{\boldsymbol{d}}}{\boldsymbol{Z}_{\boldsymbol{T}}}\right|^{2}=\frac{1+\left(\omega R_{l} C_{l}\right)^{2}}{4+\left(\omega R_{l}\right)^{2}\left(2 C_{l}+C_{d}\right)^{2}} \quad \text { and } \quad\left|\frac{\boldsymbol{Z}_{\boldsymbol{l}}}{\boldsymbol{Z}_{\boldsymbol{T}}}\right|^{2}=\frac{\left(\omega R_{l} C_{d}\right)^{2}}{4+\left(\omega R_{l}\right)^{2}\left(2 C_{l}+C_{d}\right)^{2}}
$$

with the resistance $R_{l}=h_{l} / \sigma_{l} A_{I}$, the capacitances $C_{i}=A_{I} \epsilon_{0} \epsilon_{i} / h_{i}$, and the applied frequency $\omega=2 \pi f$. Bold letters indicate complex variables, which can be out of phase with the applied potential $U_{T}$.

By combining Eq. 6.4 and 6.5 the total electric energy can now be rewritten as (See Appendix 6A3):

$$
W_{E}=-\frac{1}{2}\left(2 C_{d}\left|\frac{\mathbf{Z}_{\mathbf{d}}}{\mathbf{Z}_{\boldsymbol{T}}}\right|^{2}+C_{l}\left|\frac{\mathbf{Z}_{\mathbf{l}}}{\mathbf{Z}_{\boldsymbol{T}}}\right|^{2}\right) U_{T}^{2}=-\frac{C_{d}}{4}\left(\frac{1+\left(\omega R_{l} C_{l}\right)^{2}\left(1+\frac{C_{d}}{2 C_{l}}\right)}{1+\left(\omega R_{l} C_{l}\right)^{2}\left(1+\frac{C_{d}}{2 C_{l}}\right)^{2}}\right) U_{T}^{2}
$$

Eq. 6.6 allows the calculation of the electric energy for different parallel plate electrode geometries at different applied frequencies. It can also be used to find the critical center frequency $\omega_{c}$ between the conductive and dielectric regime. At this $\omega_{c}$ the energy gain is defined by $W_{E, t o t}=\frac{1}{2}\left(W_{E, \omega \rightarrow 0}+W_{E, \omega \rightarrow \infty}\right)$, and we find (See Appendix 6A4):

$$
\omega_{c}=\frac{1}{R_{l}\left(C_{l}+C_{d} / 2\right)}=\frac{\sigma_{l}}{\epsilon_{0}\left(\epsilon_{l}+\epsilon_{d} \frac{h_{l}}{2 h_{d}}\right)}
$$


Inspecting Eq. 6.6 more closely, we observe that it can be rewritten in the form of:

$$
W_{E}=-\frac{1}{4} c_{d}\left(\frac{1+\nu^{2} / \alpha}{1+\nu^{2} / \alpha^{2}}\right) A_{I} U_{T}^{2}
$$

with the two non-dimensional parameters

and

$$
\nu=\omega R_{l} C_{l}=\omega / \omega_{o}=\omega \epsilon_{0} \epsilon_{l} / \sigma_{l}
$$

$$
\alpha=\left(1+\frac{C_{d}}{2 C_{l}}\right)^{-1}=\left(1+\frac{\epsilon_{d} h_{l}}{2 \epsilon_{l} h_{d}}\right)^{-1}
$$

Here, $\nu$ is the applied frequency scaled with the central frequency $\omega_{0}$, which is independent of the variable $h_{l}$. And $\alpha$ is a geometry dependent factor which dictates the difference of electric energy between the conductive and dielectric regime. For a conductive liquid $(\omega \rightarrow$ 0) Eq. 6.6 can be distilled to:

$$
W_{E}=-\frac{1}{4} C_{d} U_{T}^{2}
$$

While for a pure dielectric liquid $(\omega \rightarrow \infty)$ it becomes:

$$
W_{E}=-\frac{1}{4} \alpha C_{d} U_{T}^{2}
$$

We note here that the only difference between the conductive and dielectric case is the geometric factor $\alpha$, which always has a value $<1$. Therefore, in our setup the dielectric case will always have a weaker electric energy contribution than the conductive case. The factor $\alpha$ tells us that the electric energy difference between conductive and dielectric case is determined by the ratio of capacitances of the insulating layers and the liquid.

This ratio of capacitances resembles the ratio of potentials falling across the insulator and droplet in the dielectric case. For $\alpha \rightarrow 1$, most of the electric potential falls over the insulator, similar to having a conductive droplet. While for $\alpha \rightarrow 0$, the potential largely falls over the droplet.

\subsubsection{Electric driving force}

So far we have only discussed the absolute electric energy for a given arrangement of the droplet phase. For practical purposes it is more interesting to know the force acting on a strongly polarizable liquid as it replaces a certain volume $\Delta A h_{l}$ (see Fig. 6-3) of a less polarizable ambient medium. To get a measure of this driving force the difference between the electric energy of the configuration with and without droplet has to determined (part II of Fig. 6-3) This initial contribution of the ambient medium (no droplet) and insulators we lump together and is given as:

$$
W_{e, 0}=-\frac{1}{2} C_{0} U_{T}^{2}=-\frac{1}{2} \frac{\epsilon_{d}}{2 d_{d}+d_{l}} A_{I I} U_{T}^{2}
$$


with $C_{0}$ the total capacitance of insulators and ambient medium combined. For ease the ambient medium is chosen to be an oil with same dielectric constant as the insulators. The difference in electric energy with and without drop is the electric potential energy gain $\Delta W_{E}$ :

$$
\Delta W_{E}=-\frac{1}{2}\left[\frac{c_{d}}{2}\left(\frac{1+\nu^{2} / \alpha}{1+\nu^{2} / \alpha^{2}}\right)-c_{0}\right] U_{T}^{2} \Delta A
$$

where $c_{0} / c_{d}=\left(1+h_{l} / 2 h_{d}\right)^{-1}$. This last expression (Eq. 6.14) is equivalent with a driving force (per unit width):

$$
F_{E}=\frac{c_{d}}{4}\left[\left(\frac{1+\nu^{2} / \alpha}{1+\nu^{2} / \alpha^{2}}\right)-\frac{2 h_{d}}{2 h_{d}+h_{l}}\right] U_{T}^{2}
$$

\subsubsection{Results from LEM}

To obtain an optimal connection between the LEM and our experiments, we use the parameter values as indicated in Table 6-1. The material properties correspond to the use of PDMS as insulator and propylene carbonate (PC) as the liquid. The layer thicknesses are typical values. Using Eq. 6.6, the transition from the conductive to the dielectric regime is studied by systematic variations of both the AC frequency $(f)$ and the thickness of the PC liquid $\left(h_{l}\right)$. For the potential $U_{T}$ a typical (RMS) value of $150 \mathrm{~V}$ is chosen.

For these parameters the normalized driving force per unit width $\tilde{F}_{E}[N / m]=$ $F_{E} /\left({ }_{1} C_{d} U_{T}^{2}\right)$ is plotted versus normalized frequency $\nu=2 \pi f \epsilon_{0} \epsilon_{l} / \sigma_{l}$ as the solid lines in Fig. 6-4. A first observation is that the critical frequency $\nu_{c}$ increases if $h_{l}$ becomes smaller; this is in perfect agreement with Eq. 6.7. Focusing on the conductive regime $\left(\nu \ll \nu_{c}\right)$, where the total potential falls across the insulating layers only, we find that $\tilde{F}_{E}$ decreases as $h_{l}$ decreases. This effect, which only becomes significant for $h_{l}<20 \mu m$, is solely due to the dependence of the initial background energy $W_{e, 0}$ of the ambient medium on $h_{l}$, because there is no electric field in the conductive liquid.

Looking at the dielectric regime $\left(\nu \gg \nu_{c}\right)$, where the electric field penetrates into the liquid, we observe a decrease in $\tilde{F}_{E}$ compared to the conductive regime. As explained before, this difference is caused by the factor $\alpha$ which increased with increasing $h_{l}$. Therefore, the energy decrease from conductive to dielectric regime is small for thin liquid layers, while it is relatively large for thick PC layers.

Table 6-1 Material properties and layer thicknesses used in the LEM calculations.

$\begin{array}{lll} & \text { Insulators } & \text { Liquid (PC) } \\ \text { Permittivity } \epsilon & 2.7 & 65 \text { (measured) } \\ \text { Layer thickness } h[\mu \mathrm{m}] & 2.0 & 2-1280 \\ \text { Conductivity } \sigma[\mu S / \mathrm{m}] & - & 60 \text { (measured) }\end{array}$




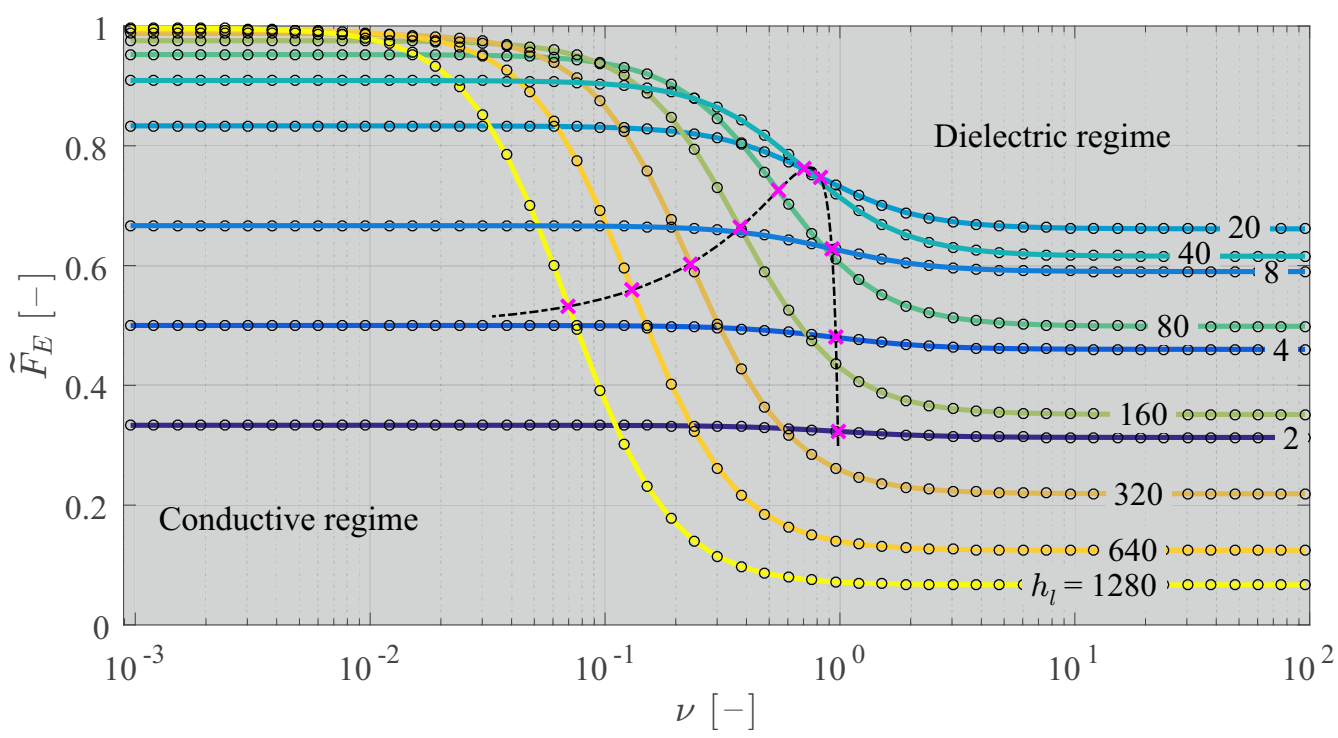

Fig. 6-4 Normalized electric force $\tilde{F}_{E}$ against normalized frequency $\nu$ for different liquid heights $h_{l}$ in the parallel plate geometry (see Fig. 6-3 for schematic). The numbers on the right indicate the height $h_{l}$ in $\mu \mathrm{m}$ for each curve. Colored lines are calculated with the analog LEM. Black circles are results from a 1 D numerical simulation (See Section 6.6). The dashed black line shows the critical frequency and the corresponding magnitude of the electric force for different liquid heights $\left(h_{l}\right)$. The magenta crosses indicate the critical frequency transition point for the selected liquid heights.

For practical applications it could be desirable to find the maximal driving force $F_{E}$ in the dielectric regime. This value can be obtained by maximizing Eq. 6.15 or equivalently by maximizing $\frac{1}{2 h_{d}+\frac{\epsilon_{d}}{\epsilon_{l}} h_{l}}-\frac{1}{2 h_{d}+h_{l}}$, as a function of $h_{l}$.

Using the values in Table $6-1$, the corresponding $h_{l}=19.6 \mu \mathrm{m}$. Indeed, Fig. $6-4$ shows the largest $\tilde{F}_{E}$ in the dielectric regime for $h_{l}=20 \mu \mathrm{m}$.

The principal outcome of the LEM calculations is clear: thinner liquid films (and thicker insulator layers) will show a smaller contrast between the driving force $F_{E}$ in the conductive and dielectric regimes. For dielectric materials the largest $F_{E}$ is obtained at a certain optimal value of $\alpha$, which depends on the ratio of the dielectric constants of the chosen materials $\epsilon_{l} / \epsilon_{d}$ and the relative heights $2 h_{d} / h_{l}$.

The LEM thus gives a clear understanding of how the electric driving force (for EW) changes with relative length scales and dielectric constants. But is only accurate when a parallel plate electrode geometry is used. For this geometry the electric fringe fields are inconsequential, since these fringe fields move along with the liquid unaltered, and thus have no influence on the electric force. For our microfluidic sorter which consists of coplanar electrodes, or the classic electrowetting setup that uses a wire as one of the electrodes, the LEM is less accurate.

For conductive liquids the LEM still works fine, since the only capacitor of interest is 
the dielectric insulator, which can still be considered a parallel plate capacitor, because the conductive liquid can be considered an electrode with uniform potential, and no electric field present in the liquid. A dielectric liquid, however, is no longer located between two parallel plates in the co-planar or classical wire-in-drop method. This means it can no longer be represented as a capacitor and resistor in parallel. Some articles use an elliptic integral to estimate the liquid capacitance, ${ }^{21}$ but this is still not very accurate, in particular when the drop is not centered above the two electrodes.

Also, for the co-planar geometry the electric fringe fields at the electrode edges have to be taken into account, in particular if the droplet contact line is near the electrode edge. But in the dielectric regime, the capacitances of the insulating layers and specifically the liquid are no longer clearly defined.

To address these shortcomings of the LEM, we need a more sophisticated, numerical model to determine the electric potential field for a droplet atop a coplanar electrode geometry. This will be covered in the next section.

\subsection{Numerical model}

The numerical model is kept relatively simple. We use the Poisson equation for electrostatics:

$$
\nabla \cdot\left(\epsilon_{r} \nabla \phi\right)=-\rho_{E}
$$

which gives the relation between charge density $\rho_{E}$ and electric potential $\phi$, depending on the permittivity of the medium $\epsilon_{r}$. Further, we take into account charge conservation:

$$
-\frac{\partial \rho_{E}}{\partial t}=\nabla \cdot \boldsymbol{J}
$$

Here the change of charge density $\rho_{E}$ over time, is related to the divergence of the current density:

$$
\boldsymbol{J}=-\sigma \nabla \phi
$$

Combining these two equations we end up with a second order differential equation:

$$
\nabla \cdot\left(\epsilon_{r} \nabla \dot{\phi}\right)=\nabla \cdot(-\sigma \nabla \phi)
$$

Assuming a sinusoidal potential:

$\phi=\phi_{0} R e\left[e^{i \omega t}\right]$, and thus $\dot{\phi}=\phi_{0} R e\left[i \omega e^{i \omega t}\right]$,

the differential equation becomes:

$$
\nabla \cdot\left(\left(\epsilon_{0} \epsilon_{r}-\frac{i \sigma}{\omega}\right) \nabla \phi\right)=0
$$


(a)

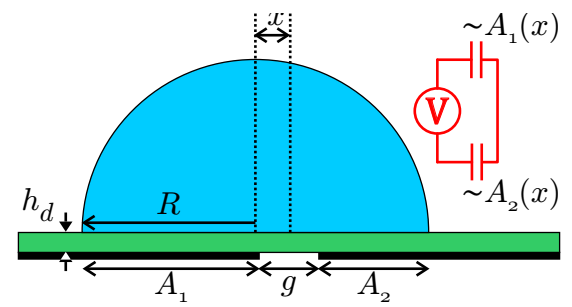

(b)

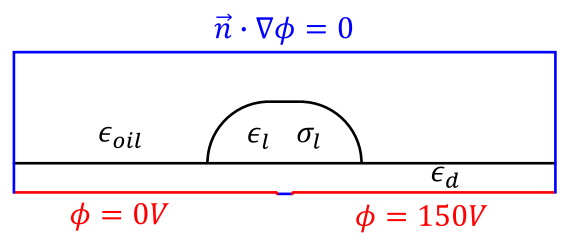

Fig. 6-5 (a) Schematic representation of electrowetting on a co-planar electrode geometry. For simplicity we consider the drop as a 2D semicircular cap. Red schematic shows the corresponding electronic circuit assuming a conductive droplet. (b) Boundary conditions as applied in the numerical simulation.

The boundary conditions on the electric potential $\phi$ are Dirichlet conditions for dictating the potential on the electrodes, and Neumann conditions for all the other outer edges. (See Fig. 6-5b) Solving the PDE using COMSOL software gives the distribution of the potential field. Once the potential field is numerically determined, the total electric energy $W_{E}$ stored in the system and averaged over one oscillation cycle, can be calculated with a volume integral over the entire domain:

$$
W_{E}=-\frac{1}{2} \epsilon_{0} \int \epsilon_{r}\left(\left|\frac{\partial \phi_{r m s}}{\partial x}\right|^{2}+\left|\frac{\partial \phi_{r m s}}{\partial y}\right|^{2}+\left|\frac{\partial \phi_{r m s}}{\partial z}\right|^{2}\right) d V
$$

with $\phi_{r m s}=\phi_{0} / \sqrt{2}$. Similar to before (Section 6.5.2), $\Delta W_{E}$ is determined by subtracting the initial electric energy, which is determined from a numerical calculation in absence of a droplet.

\subsubsection{Validation of model}

To determine whether the numerical model is capable of calculating the electric energy, it is first executed in 1-D using the same parameters as used for the LEM in Section 6.5.3. The initial electric energy is also numerically determined and subtracted. The resulting values for the electric force per unit width $F_{E}=-\Delta W_{E} / \Delta A$ are superimposed on Fig. 6-4c (black circles). As can be seen, the analog LEM and numerical PDE give the same result.

\subsection{Numerical Results}

We now analyze a 2-D coplanar electrode geometry, as shown in Fig. 6-6a. Here, the liquid is a semicircular cap $(\theta=\pi / 2)$ that can be positioned a distance $x$ with respect to the center of the gap $g$ between the electrodes. The $x$-position determines the size of the areas $A_{1}(x)$ and $A_{2}(x)$ that the liquid covers over each of the electrodes. This allows us to calculate the electric energy at each position, which in turn allows calculation of the electric force exerted on the liquid: $F_{E}=-\Delta W_{E} / \Delta x$. 
So, in 2-D it is quite simple to calculate the analytical result of the conductive case using the LEM. Since we know from the parallel plate analysis that the conductive case will have the strongest electric force, this allows us to normalize the numerical results by dividing by the results from the LEM for a conductive droplet. This in turn allows us to compare the numerical results while changing geometric parameters like the insulator thickness $h_{d}$ and the droplet radius $R$, which should play a similar role as the liquid thickness $h_{l}$ from the parallel plate geometry.

\subsubsection{Electric energy}

In Fig. 6-6a the non-dimensional electric energy $\widetilde{W}_{E}$ is plotted against the non-dimensional position with respect to the gap $\tilde{x}$. (See section 6.7.3 and 6.7.4 for normalization method.) The numerically generated data are calculated for insulator thicknesses $h_{d}$ of $0.5,2.5$ and $10 \mu \mathrm{m}$ and for a conductive $\left(\omega \ll \omega_{c}\right)$ and dielectric $\left(\omega \gg \omega_{c}\right)$ droplet, while keeping the gap width $g=10 \mu \mathrm{m}$, contact radius $R=50 \mu \mathrm{m}$ and contact angle $\theta=\pi / 2$ constant. We observe that the conductive cases follow the analog model well for each insulator thickness. For the thicker insulator we do find a wider, more gradual curve extending beyond $\tilde{x}=1$. This is because a thicker insulator allows the drop to be affected the electric field further away from the gap, due to fringe effects of the field distribution that are not taken into account in the LEM modeling.

For the dielectric cases there is a reduction in electric energy relative to the conductive cases. For the thick insulator this difference is only small. With a thin insulator we see a much larger relative reduction in electric energy, and also a change in the overall shape of the curve. The energy curve is flat when the droplet is near the center, yet lowers reasonably steeply where the edge of the drop reaches the edge of the electrode. This is caused by the radially extending electric field at these sharp corners, causing strong gradients much more notably 'felt' for thin insulators, and which will locally affect the electric force exerted on the droplet.

In Fig. 6-6b the numerically calculated normalized electric energy is plotted once again, but now the insulator thickness $h_{d}$ is kept constant at $2.5 \mu \mathrm{m}$, while the liquid radius $R$ is set to 25,50 , and $100 \mu \mathrm{m}$. Again we notice that the conductive cases overlap nicely, except beyond $\tilde{x}=1$. Now the small radius liquid feels the electric field slightly further away from the electrode gap, since the insulator thickness is relatively larger compared for the small radius. For the dielectric cases the reduction in electric energy is largest for the liquid with the largest radius. In Appendix 6B images are shown of the numerically determined electric potential field for each combination of $h_{d}$ and $R$ as described above. Also the electric field lines are shown.

\subsubsection{Electric force}

To make a closer connection with the droplet sorting experiments, it is more appropriate to compare the electric forces exerted on the droplet in the conductive and dielectric regimes. The electric force can be determined by taking the derivative of the electric energy with respect to position: $F_{E}=-\Delta W_{E} / \Delta x$. The numerically calculated normalized forces for altering insulator thicknesses are shown in Fig. 6-6c. We note that for the thin insulator, significant differences are found between the conductive and dielectric cases. 
For the conductive droplet, the numerical results follow the straight line of the 2D LEM reasonably well. For the thick insulator we explained before that the energy change is more gradual as the drop is moving across the gap. This results in a lower force for increasing insulator thickness.

For dielectric liquid we see a much weaker force near the center of the gap. However, as the liquid edge moves over the gap, the strong gradient in the electric field caused by the electrode edge causes an increase in the electric force. For the thick insulator that means there is actually little difference between the conductive and dielectric case. Comparing the dielectric cases, we find that the maximum value of the normalized electric forces for a thick and thin insulator are actually not very different.
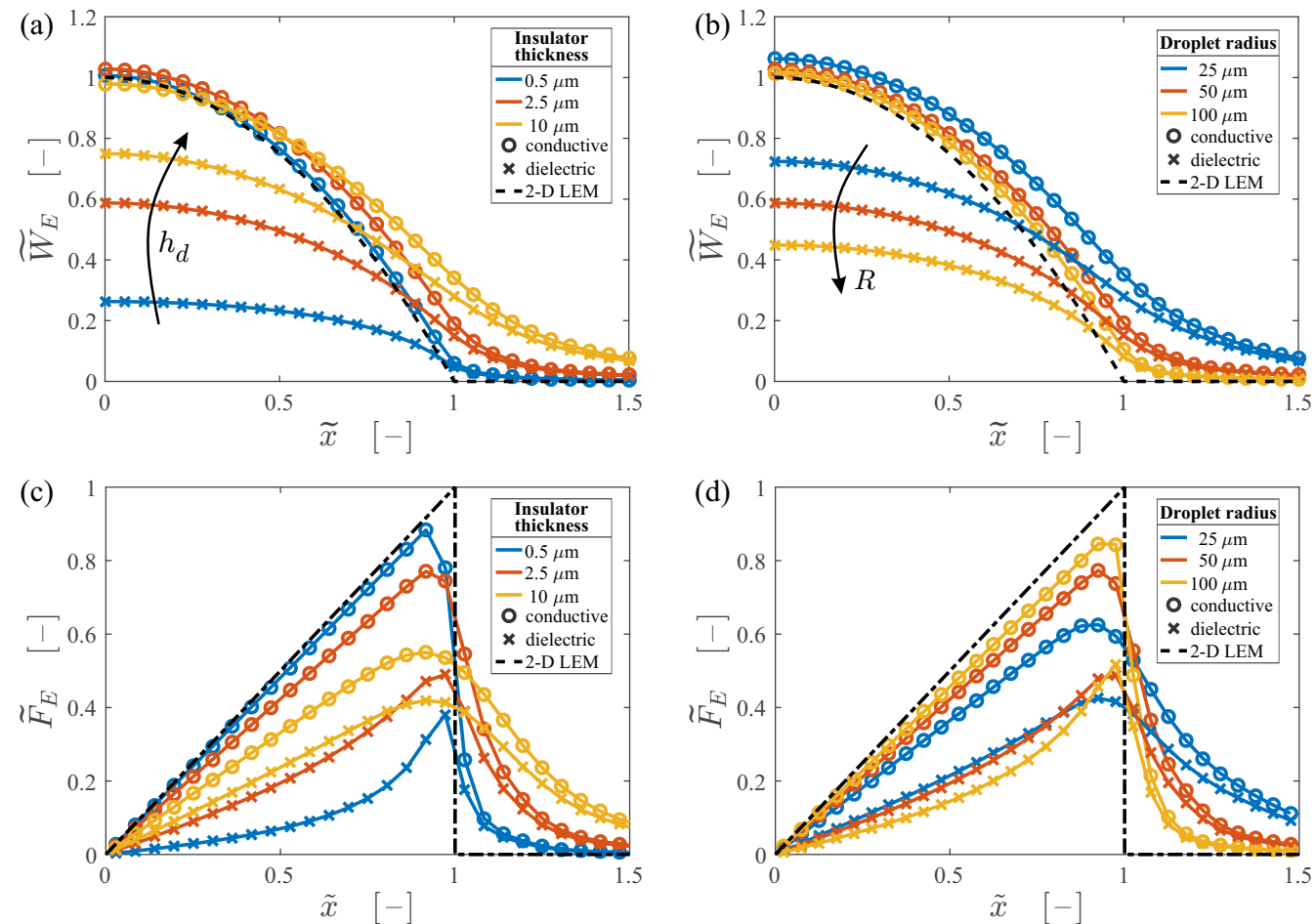

Fig. 6-6 (a) Non-dimensionalized electric energy against the droplet position numerically calculated for the conductive and dielectric regime, and for large and small geometric ratio $\left(h_{d} / R\right)$. The black parabola shows the theoretical prediction of the $2 D$ analog LEM, i.e. $\widetilde{W}_{E}=1-\tilde{x}^{2}$. The insulator thickness is varied using a constant drop radius of 50 $\mu \mathrm{m}$. In (b) the drop radius is varied using a constant $2.5 \mu \mathrm{m}$ insulator thickness. (c) Non-dimensionalized electric force against the droplet position numerically calculated for the conductive and dielectric regime, and for large and small geometric ratio $\left(h_{d} / R\right)$. Black line shows the theorical prediction of the $2 D$ analog $L E M \tilde{F}_{E}=\tilde{x}$. The insulator thickness is varied using a $50 \mu \mathrm{m}$ drop radius. In (d) the drop radius is varied with a constant $2.5 \mu \mathrm{m}$ insulator thickness. 
In Fig. 6-6d the electric forces for altering radius are plotted. We observe that for conductive liquids a larger radius causes a larger force. For the dielectric cases there is little difference. The larger radius does have a lower force near the gap, but near the edge the maximum force is comparable.

\subsubsection{Normalization of electric energy}

The magnitude of the electric energy vary strongly when altering parameters such as the thickness of the dielectric layer, or the dielectric constants. To allow comparing the electric energy contribution of different co-planar geometries, we normalize the electric energy with $W_{0}$, the latter being the analog result of a 2-D lumped element model for a conductive droplet at $x=0$ (See Fig. 6-5a). We start with the expression for the position dependent electric energy for a conductive droplet moving over coplanar electrodes, where $C_{T}$ is the combined capacitance of the insulators, given as on $\frac{\epsilon_{0} \epsilon_{d}}{h_{d}} A_{1}$ and $\frac{\epsilon_{0} \epsilon_{d}}{h_{d}} A_{2}$ :

$W_{E}=-\frac{1}{2} C_{T} U^{2}=-\frac{\epsilon_{0} \epsilon_{d}}{2 h_{d}} \frac{A_{1} A_{2}}{A_{1}+A_{2}} U^{2}$

with $A_{1}=b(a-x)$ and $A_{2}=b(a+x)$,

with $a=R-\frac{g}{2}$, and $b$ the considered width of the drop.

Filling in the expressions, the factor $\frac{A_{1} A_{2}}{A_{1}+A_{2}}$ can be rewritten as $\frac{a b}{2}\left(1-\frac{x^{2}}{a^{2}}\right)$, which contains a constant $(a b / 2)$ and an $x$ dependent part $\left(1-\frac{x^{2}}{a^{2}}\right)$. For normalization we can now chose:

$\widetilde{W}_{E}=W_{E} / W_{0}$ with $W_{0}=-\frac{a b}{2} \frac{\epsilon_{0} \epsilon_{r}}{2 h_{d}} U^{2}$, and $\tilde{x}=\frac{x}{a}$.

This leads to the simple parabolic function $\widetilde{W}_{E}=1-\tilde{x}^{2}$ for $\tilde{x}<1$.

\subsubsection{Normalization of electric force}

Normalization similar to the energy case leads to the linear function:

$$
\tilde{F}_{E}=\tilde{x} \text { with } \tilde{F}_{E}=\frac{a}{2 W_{0}} F_{E} \text { and } \tilde{x}=\frac{x}{a} \text { for } \tilde{x}<1
$$

\subsection{Electrowetting with dielectric liquids}

For classical electrowetting experiments with a single insulator-covered electrode and a ground wire in the droplet as the counter electrode (see Fig. 6-1a), the electric energy of the system does not change upon lateral movement of the liquid. However, the contact radius, and thus the contact angle, does adapt - at least for conductive droplets - to minimize the sum of the electric and surface energies. This highlights a simplification that was made in the numerical model: the shape of the droplet was kept constant. A more complete numerical analysis allowing both center-of-mass displacement and droplet deformation would however involve rather intensive computations without significantly changing the findings for the position dependent force. For electrowetting on co-planar 
electrodes, positional changes of the droplet would not need to be considered, because unlike the sorting experiment where there is a balance with a hydrodynamic force - the restoring force (shown in Fig. 6-6c and d) would always keep the droplet centered above the gap at $x=0$.

An approximate way to examine the effect of a transition from the conductive to the dielectric regime on the contact angle is to use the electric energy at $x=0$. Since the background energy (in absence of the droplet) is constant, changes in the electric energy of the system can also be regarded as changes in the electric contact line force for electrowetting. Increases in $\Delta W_{E}$ caused by a change in parameters should directly lead to lower contact angles (and vice versa for decreases in $\Delta W_{E}$ ). This means that the relative change in contact angle as the droplet transitions from conductive to dielectric, can still be predicted. The results in Fig. 6-6b already indicate that the most dramatic changes in contact angle, i.e the most dramatic losses of electrowetting, are to be expected for dielectric liquids above a thin insulator.

\subsubsection{Experimental results}

From the analog model, supported by the numerical model, we found that the factor $\alpha$ represents the proportion of electric energy in the dielectric regime compared to the conductive case. Knowing the parameters that govern the frequency-dependent effectivity of electrowetting, it is possible to design experiments where electrowetting remains effective in the dielectric regime. The first change is to increase $h_{d}$ by using a thick insulating layer of $12 \mu \mathrm{m}$, which in regular experiments lies around $100 \mathrm{~nm}$ to $2 \mu \mathrm{m}$. The second change is decreasing $h_{l}$ by using a smaller droplet radius. Even though $h_{l}$ is not clearly defined for droplets atop a co-planar electrode geometry, its value should be comparable to the radius of the droplet, albeit slightly larger since the electric field lines take an elliptical path through the droplet.

Fig. 6-7a shows snapshots of a small $(0.06 \mu L)$ droplet of propylene carbonate in bromohexadecane atop a coplanar electrode geometry insulated by a $12 \mu \mathrm{m}$ SU8 layer hydrophobized with a $\sim 50 \mathrm{~nm}$ spincoated layer of Cytop. The gap between the electrodes is $50 \mu \mathrm{m}$. A potential of $150 \mathrm{~V}$ is applied at logarithmically increasing AC frequencies ranging from $10^{2.6}$ to $10^{5} \mathrm{~Hz}$.

Fig. 6-7b shows the experimentally measured change in contact angle $\Delta \cos \theta=$ $\cos \theta_{e w}-\cos \theta_{0 V}$ against the applied $\mathrm{AC}$ frequency for droplets of different volumes. Droplet volumes of $0.06,0.68,3.1$ and $9.8 \mu L$ are used, which corresponds to values for $\alpha$ of $0.65,0.45,0.33$ and 0.25 , respectively, assuming $R=h_{l}$. Two effects can be observed when the volume of the droplet decreases, or in other words, when $h_{l}$ becomes smaller. The critical frequency $f_{c}$ goes up, as predicted by Eq. 6.7. Also, while the contact angle change in the conductive regime is similar for all droplet sizes, $\Delta \cos \theta$ in the dielectric regime is clearly different. By decreasing the droplet size, we have increased the efficiency of EW in the dielectric regime.

One interesting observation from Fig. $6-7$ is that $\Delta \cos \theta$ slightly rises at the initial transition frequency from the conductive to the dielectric regime, something which is not observed in classical electrowetting. This rise has recently been observed more strongly in DMF devices that use interdigitated electrodes. ${ }^{22}$ It therefore seems plausible that it is 
caused by the presence of electrode edges and the resulting strong electric field gradients at these edges.
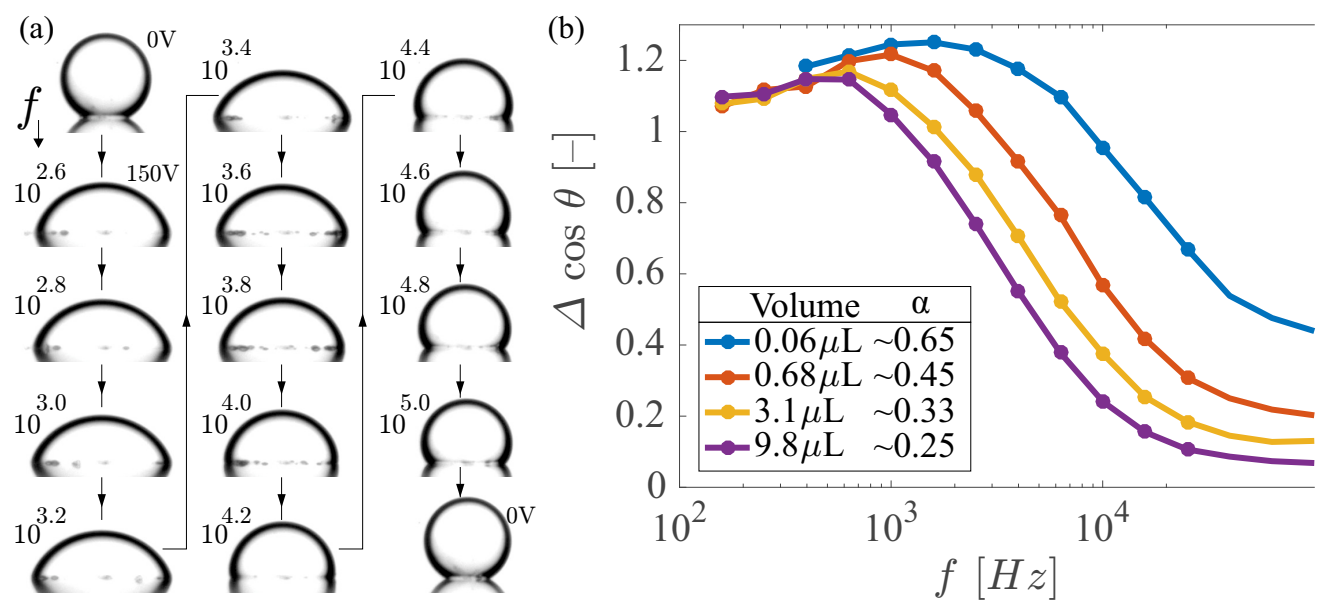

Fig. 6-7 (a) electrowetting experiment for measuring the change in contact angle of a $0.06 \mu \mathrm{L}$ propylene carbonate droplet in bromohexadecane on a coplanar electrode geometry

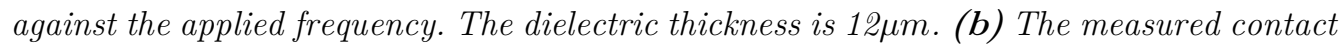
angle versus applied frequency for different droplet volumes. As can be seen, for smaller volumes (and thus larger $\alpha$ ) the electrowetting effect is stronger in the dielectric regime.

\subsection{Discussion}

We observed two apparently contradictive behaviors: on one hand, a droplet in a classical needle-in-drop EW geometry shows a strong decrease in electric force in the dielectric regime, as indicated by a lack of contact angle change, while on the other hand, a droplet in a microfluidic sorter showed hardly any decrease in electric force. Use of a lumped element model (LEM) describing the parallel plate geometry is sufficient to understand that the decrease in electric energy in the dielectric regime lies in relative geometric length scales and dielectric contrast as dictated by the factor $\alpha=\left(1+\epsilon_{d} h_{l} /\left(2 \epsilon_{l} h_{d}\right)\right)^{-1}$.

For complex geometries the LEM is not accurate in the dielectric regime where the insulators and liquid can no longer be described as parallel plate capacitors. The factor $\alpha$ was easily determined for the parallel plate geometry since the capacitances of all the material volumes involved are known. Unless the electric field distribution is known (for instance from numerical simulation), we cannot determine the effective capacitance of the non-conductive liquid for the needle-in-drop or co-planar geometry. We can, however, use the qualitative argument that in the classical EW geometry the droplet is relatively large compared to the insulator thickness, let's say in the order of millimeters versus micrometers. In this geometry the distance from insulator to grounding wire in the droplet is comparable to the liquid length scale $h_{l}$ as defined in the parallel plate geometry. Similarly, for a droplet on a co-planar electrode geometry, the radius $R$ is - in a non-exact sense - comparable to the liquid length scale $h_{l}$. Therefore, a larger droplet effectively 
decreases the factor $\alpha$ causing a decrease of electrowetting force.

Thus, a relatively large droplet will have a relatively low effective capacitance compared to the insulating layer. Since the droplet and insulating layer are in series this results in the applied potential mostly falling over the big droplet. This in turn means a stronger electric field (and therefore the larger portion of the electric energy) is present inside the droplet. In this case, increasing the contact area between droplet and insulator layer does not produce enough electric energy gain, to compensate for the increase in surface energy. The contact angle therefore hardly changes.

In the case of the microfluidic sorter the droplets are much smaller. Given that the insulator layer thickness is similar to classical EW experiments, the ratio between the lengthscales of the liquid and the insulator $h_{d} / h_{l}$ is now much smaller, while the permittivity ratio $\epsilon_{l} / \epsilon_{d}$ is still large. This means the effective capacitance of the liquid is larger than that of the insulators, resulting in the electric field still being located primarily inside the insulator, similar to having a conductive liquid. In fact, comparison with the results from the parallel plate LEM shown in Fig. 6-4 indicated that the optimum droplet size $\left(h_{l}=20 \mu m\right)$ and the droplet radius in the microchannel $(R \sim 50 \mu m$, handwavingly comparable to $h_{l}$ ) are similar. This explains why the electric forces were strong, even in the dielectric regime, leading to successful sorting.

As mentioned, for complex geometries the LEM is not very accurate in the dielectric regime. In these cases the numerical model offers significant improvements. It allows accurate calculation of electric energy for different electrode geometries, different droplet positions and variable length scales of the insulator and droplet. Furthermore, the numerical model takes into account fringe fields at the electrode and droplet edges, which are important if the drop size and insulating layer thickness become comparable.

The results from the numerical model indeed show differences with the LEM, mainly resulting from fringe effects. In the conductive regime, and with a thin insulator, the LEM and numerical model match rather well, and therefore the simple LEM is most useful in designing the geometrical parameters of an electrowetting setup. For thicker insulators, the force calculated from the LEM is overestimated, because a thicker insulator results in a more gradual transitions when a droplet moves over the electrode gap. This leads to a wider electric energy curve against drop position, which in turn results in a smaller normalized electric force.

We observe in Fig. 6-6c that for the dielectric cases, the shapes of the normalized force curves for a thick and thin insulator are different. Still, because of the strong electric fields at the electrode edges the maximum normalized electric force, achieved at the x-position where the droplet leaves one of the electrodes, is similar for all insulator thicknesses. For the microfluidic sorting of droplets this maximum electric force is the most important parameter, which means that the maximum sorting rate remains comparable for all chosen geometric parameters. For other geometries, e.g. a DMF setup which is comparable to the parallel plate geometry, these fringe effects do not assist the movement of the droplet, and the force remains constant as the droplet moves over the actuated electrode. Here, a thicker insulator will be better at transporting dielectric liquids.

To find the electric forces for practical applications, the curves of the electric forces in Fig. 6-6c have to be multiplied by the scaling factor. While this means that the force will 
become weaker for thicker insulator layers, this trend can be more than compensated via the possibility to use an increased voltage, because the threshold voltage for dielectric breakdown in general scales linearly with the insulator thickness. Since the electric force is proportional to the squared voltage ${ }^{23}$ this means that a thicker insulator can actually generate a much larger electric force as long as the voltage amplifier can handle the increased demand.

Ultimately in experiments with dielectric liquids on thin insulating layers it might be unwise to rely on the fringe electric fields present at the edge of electrodes for actuating droplets. The large electric fields could be prone to dielectric breakdown.

\subsection{Conclusion}

We have shown that the apparently contradicting observations when using EWOD to actuate droplets in the dielectric regime, can be understood by looking carefully at the material properties and length scales of all the elements in the electrowetting configuration. A simple lumped element model was used to explain how the electric energy gain obtained by electrowetting depends on thicknesses, dielectric constants, liquid conductivity and AC frequency. This allowed to identify which regime (conductive or dielectric) is addressed in experiments, and also how the driving force for EW depends on the $\mathrm{AC}$ frequency. For a more accurate determination of electric energy gain, a finite element model was designed, and validated in $1 \mathrm{D}$ by comparison with the LEM. Application of the 2D numerical model to the case of droplets on coplanar EWOD electrodes yielded a quantitative insight in how the electrostatic trapping force depends on geometry in both the conductive and dielectric regimes, and an explanation for the experimentally observed possibility to even sort dielectric droplets. Also the qualitative dependence of the contact angle change on the AC frequency could be extracted from the numerical 2D model. It gave a good correspondence with our experimental observations.

The LEM or numerical models should be helpful for designing electrowetting setups, especially when dielectric liquids need to be actuated. 


\section{Appendix 6A: The lumped element model}

\section{A1 Describing the parallel plate system}

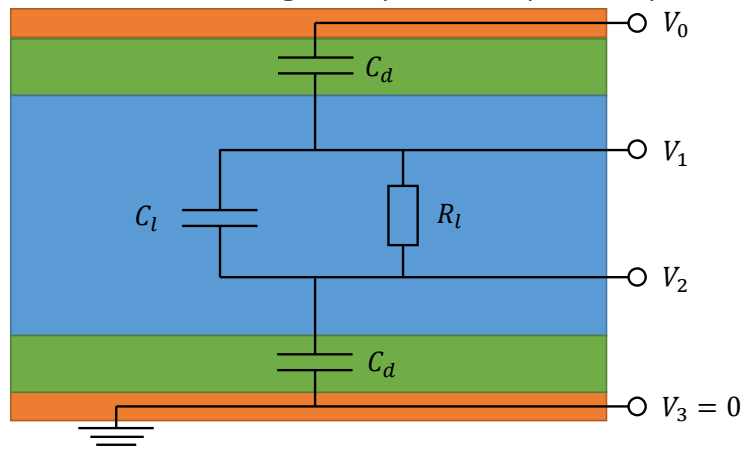

Definitions for potential and impedance:

$$
\begin{aligned}
& V_{0}=Z_{t o t} I \quad V_{0}-V_{1}=V_{2}=\frac{1}{j \omega C_{d}} I \quad V_{1}-V_{2}=\left(\frac{1}{R_{l}}+j \omega C_{l}\right)^{-1} I \\
& Z_{t o t}=2 Z_{d}+Z_{l}=\frac{2}{j \omega C_{d}}+\frac{R_{l}}{1+j \omega R_{l} C_{l}}
\end{aligned}
$$

General rules for charge on capacitors:

$$
Q=C V \quad \& \quad W_{E}=\frac{1}{2} Q V \quad \rightarrow \quad W_{E}=\frac{1}{2} C V^{2}
$$

The total electric energy of the system is the energy supplied by the battery and the energy stored in the insulating layers and liquid:

$$
W_{E, t o t}=W_{E}+W_{E, b a t}
$$

First we determine the electric energy in the two insulating layers:

$Q_{d}=C_{d} V_{2}$ with $V_{2}=\frac{Z_{d}}{2 Z_{d}+Z_{1}} V_{0}$ then

$2 * W_{E, d}=Q_{d} V_{2}=C_{d}\left|\frac{Z_{d}}{2 Z_{d}+Z_{l}}\right|^{2} V_{0}^{2} \cos ^{2}(\omega t+\zeta)=C_{d}\left|\frac{Z_{d}}{2 Z_{d}+Z_{l}}\right|^{2} V_{e f f}^{2}$

where $V_{\text {eff }}$ is the RMS value of $V_{0}$. And for the electric energy in the complex liquid, where the potential $V_{1}-V_{2}=\frac{Z_{l}}{2 Z_{d}+Z_{l}} V_{0}$, we get:

and thus

$$
Q_{l}=C_{l}\left(V_{1}-V_{2}\right)=C_{l} \frac{Z_{l}}{2 Z_{d}+Z_{l}} V_{0}
$$

$$
W_{E, l}=\frac{1}{2} Q_{l}\left(V_{1}-V_{2}\right)=\frac{1}{2} C_{l}\left|\frac{Z_{l}}{2 Z_{d}+Z_{l}}\right|^{2} V_{e f f}^{2}
$$


The total energy stored in the capacitors is now the sum of all electric field energy:

$$
W_{E}=\left[C_{d}\left|\frac{Z_{d}}{2 Z_{d}+Z_{l}}\right|^{2}+\frac{1}{2} C_{l}\left|\frac{Z_{l}}{2 Z_{d}+Z_{l}}\right|^{2}\right] V_{e f f}^{2}
$$

To charge the capacitors the battery has to provide the charges to the top plate $(Q=$ $\left.C_{d} V_{2}\right)$. To do so the battery has expended electric energy:

$$
W_{E, \text { bat }}=-C_{d} V_{2} V_{0}=-C_{d}\left|\frac{Z_{d}}{2 Z_{d}+Z_{l}}\right| V_{0} \cos (\omega t+\zeta) V_{0} \cos (\omega t)=-C_{d} \operatorname{Re}\left\{\frac{Z_{d}}{2 Z_{d}+Z_{l}}\right\} V_{e f f}^{2}
$$

The total electric energy of the entire system therefore becomes:

$$
W_{E, t o t}=W_{E}+W_{E, b a t}=\left[C_{d}\left\{\left|\frac{Z_{d}}{2 Z_{d}+Z_{l}}\right|^{2}-\operatorname{Re}\left\{\frac{Z_{d}}{2 Z_{d}+Z_{l}}\right\}\right\}+\frac{1}{2} C_{l}\left|\frac{Z_{l}}{2 Z_{d}+Z_{l}}\right|^{2}\right] V_{e f f}^{2}
$$

Which, in our case when the liquid displaces the ambient medium, has a negative value, indicating energy was put into the system by the battery.

\section{A2 The sum of electric field energy equals the total electric energy}

For a single capacitor charged by a battery we know that the energy stored in the capacitor is half of the energy provided by the battery, while the other half is lost to heat (or radiation). Our assumption is that even in our system with a complex liquid the energy stored in the electric fields of the capacitors is half the energy provided by the battery, thus $-W_{E, \text { bat }} / 2=W_{E}$.

In other words, we have to prove that in this case the total electric energy gained to our system $W_{E \text {,tot }}$, which is defined as the energy supplied by the battery $W_{E \text {,bat }}$ plus the energy stored in electric fields $W_{E}$, equals minus the energy stored in the electric field. Thus, $W_{E, t o t}=W_{E}+W_{E, \text { bat }}=-W_{E}$ has to hold.

To verify this assumption, for which we first need to find expressions for the impedances ratios described before:

$$
\begin{gathered}
\frac{Z_{d}}{2 Z_{d}+Z_{l}}=\frac{1 / j \omega C_{d}}{\frac{2}{j \omega C_{d}}+\frac{R_{l}}{1+j \omega R_{l} C_{l}}}=\frac{\left(1+j \omega R_{l} C_{l}\right)}{2+j \omega R_{l}\left(2 C_{l}+C_{d}\right)} \\
\frac{Z_{l}}{2 Z_{d}+Z_{l}}=\frac{\frac{R_{l}}{1+j \omega R_{l} C_{l}}}{\frac{2}{j \omega C_{d}}+\frac{R_{l}}{1+j \omega R_{l} C_{l}}}=\frac{j \omega R_{l} C_{d}}{2+j \omega R_{l}\left(2 C_{l}+C_{d}\right)}
\end{gathered}
$$


Which result in:

$\left|\frac{Z_{d}}{2 Z_{d}+Z_{l}}\right|^{2}=\frac{\left(1+j \omega R_{l} C_{l}\right)}{2+j \omega R_{l}\left(2 C_{l}+C_{d}\right)} * \frac{\left(1-j \omega R_{l} C_{l}\right)}{2-j \omega R_{l}\left(2 C_{l}+C_{d}\right)}=\frac{1+\left(\omega R_{l} C_{l}\right)^{2}}{4+\left(\omega R_{l}\right)^{2}\left(2 C_{l}+C_{d}\right)^{2}}$

$\left|\frac{Z_{l}}{2 Z_{d}+Z_{l}}\right|^{2}=\frac{j \omega R_{l} C_{d}}{2+j \omega R_{l}\left(2 C_{l}+C_{d}\right)} * \frac{-j \omega R_{l} C_{d}}{2-j \omega R_{l}\left(2 C_{l}+C_{d}\right)}=\frac{\left(\omega R_{l} C_{d}\right)^{2}}{4+\left(\omega R_{l}\right)^{2}\left(2 C_{l}+C_{d}\right)^{2}}$

$\operatorname{Re}\left\{\frac{Z_{d}}{2 Z_{d}+Z_{l}}\right\}=\operatorname{Re}\left\{\frac{\left(1+j \omega R_{l} C_{l}\right)}{2+j \omega R_{l}\left(2 C_{l}+C_{d}\right)} * \frac{2-j \omega R_{l}\left(2 C_{l}+C_{d}\right)}{2-j \omega R_{l}\left(2 C_{l}+C_{d}\right)}\right\}=\frac{2+\left(\omega R_{l}\right)^{2} C_{l}\left(2 C_{l}+C_{d}\right)}{4+\left(\omega R_{l}\right)^{2}\left(2 C_{l}+C_{d}\right)^{2}}$

The statement $-W_{E, b a t} / 2 \equiv W_{E}$ after implementing the found impedance ratios now boils down to:

$$
\begin{aligned}
\frac{1}{2} C_{d} \operatorname{Re}\left\{\frac{Z_{d}}{2 Z_{d}+Z_{l}}\right\} & =C_{d}\left|\frac{Z_{d}}{2 Z_{d}+Z_{l}}\right|^{2}+\frac{1}{2} C_{l}\left|\frac{Z_{l}}{2 Z_{d}+Z_{l}}\right|^{2} \\
\frac{1}{2} C_{d} \frac{2+\left(\omega R_{l}\right)^{2} C_{l}\left(2 C_{l}+C_{d}\right)}{4+\left(\omega R_{l}\right)^{2}\left(2 C_{l}+C_{d}\right)^{2}} & =C_{d} \frac{1+\left(\omega R_{l} C_{l}\right)^{2}}{4+\left(\omega R_{l}\right)^{2}\left(2 C_{l}+C_{d}\right)^{2}}+\frac{1}{2} C_{l} \frac{\left(\omega R_{l} C_{d}\right)^{2}}{4+\left(\omega R_{l}\right)^{2}\left(2 C_{l}+C_{d}\right)^{2}} \\
\frac{1}{2} C_{d}\left[2+\left(\omega R_{l}\right)^{2} C_{l}\left(2 C_{l}+C_{d}\right)\right] & =C_{d}\left[1+\left(\omega R_{l} C_{l}\right)^{2}\right]+\frac{1}{2} C_{l}\left(\omega R_{l} C_{d}\right)^{2} \\
C_{d}+\left(\omega R_{l}\right)^{2} C_{l} C_{d}\left(C_{l}+\frac{1}{2} C_{d}\right) & =C_{d}+\left(\omega R_{l}\right)^{2} C_{l} C_{d}\left(C_{l}+\frac{1}{2} C_{d}\right)
\end{aligned}
$$

Q.E.D.

\section{A3 Electric energy}

And thus we can simply use $W_{E, t o t}=-W_{E}$ to calculate the total electric energy gain, without having to take into account the battery contribution. $W_{E, t o t}$ now can be written as:

$$
\begin{aligned}
W_{E, \text { tot }} & =-\left[C_{d} \frac{1+\left(\omega R_{l} C_{l}\right)^{2}}{4+\left(\omega R_{l}\right)^{2}\left(2 C_{l}+C_{d}\right)^{2}}-\frac{1}{2} C_{l} \frac{\left(\omega R_{l} C_{d}\right)^{2}}{4+\left(\omega R_{l}\right)^{2}\left(2 C_{l}+C_{d}\right)^{2}}\right] V_{\text {eff }}^{2} \\
& =-C_{d} \frac{1+\left(\omega R_{l}\right)^{2} C_{l}^{2}+\frac{1}{2}\left(\omega R_{l}\right)^{2} C_{l} C_{d}}{4+\left(\omega R_{l}\right)^{2}\left(2 C_{l}+C_{d}\right)^{2}} V_{e f f}^{2} \\
& =-C_{d} \frac{1+\frac{1}{2}\left(\omega R_{l}\right)^{2} C_{l}\left(2 C_{l}+C_{d}\right)}{4+\left(\omega R_{l}\right)^{2}\left(2 C_{l}+C_{d}\right)^{2}} V_{e f f}^{2} \\
& =-\frac{1}{4} C_{d} \frac{1+\left(\omega R_{l}\right)^{2} C_{l}\left(C_{l}+C_{d} / 2\right)}{1+\left(\omega R_{l}\right)^{2}\left(C_{l}+C_{d} / 2\right)^{2}} V_{e f f}^{2} \\
& =-\frac{1}{4} C_{d} \frac{1+\left(\omega R_{l} C_{l}\right)^{2}\left(1+C_{d} / 2 C_{l}\right)}{1+\left(\omega R_{l} C_{l}\right)^{2}\left(1+C_{d} / 2 C_{l}\right)^{2}} V_{e f f}^{2}
\end{aligned}
$$


This equation for $W_{E, t o t}$ can be rewritten in the form of:

With

$$
W_{E, t o t}=-\frac{1}{4} C_{d} V_{e f f}^{2}\left[\frac{1+\nu^{2} / \alpha}{1+\nu^{2} / \alpha^{2}}\right]
$$

$\nu \equiv \omega R_{l} C_{l} \quad$ and $\quad \alpha=\left(1+\frac{C_{d}}{2 C_{l}}\right)^{-1}=\left(1+\frac{\epsilon_{d} h_{l}}{2 \epsilon_{l} h_{d}}\right)^{-1}$

What information can be extracted from this equation? We get the expression for the electric energy in the conductive regime, let $\omega \rightarrow 0$ :

$$
W_{E, \omega \rightarrow 0}=-\frac{1}{4} C_{d} V_{e f f}^{2}
$$

And we get the expression for the electric energy in the dielectric regime, $\omega \rightarrow \infty$ :

$$
W_{E, \omega \rightarrow \infty}=-\frac{1}{4} \alpha C_{d} V_{e f f}^{2}
$$

\section{A4 Critical frequency}

The critical transition frequency can be found at the value of $W_{E, t o t}$ halfway between $W_{E, \omega \rightarrow 0}$ and $W_{E, \omega \rightarrow \infty}$. Thus when

$$
\begin{aligned}
W_{E, \text { tot }} & =\frac{W_{E, \omega \rightarrow 0}+W_{E, \omega \rightarrow \infty}}{2} \\
-\frac{1}{4} C_{d} V_{\text {eff }}^{2}\left[\frac{1+\nu^{2} / \alpha}{1+\nu^{2} / \alpha^{2}}\right] & =-\frac{1}{4} C_{d} V_{\text {eff }}^{2}\left(\frac{1}{2}+\frac{\alpha}{2}\right) \\
1+\nu^{2} / \alpha & =\frac{1}{2}+\frac{\alpha}{2}+\frac{\nu^{2}}{2 \alpha^{2}}+\frac{\nu^{2}}{2 \alpha} \\
1-\nu^{2} / \alpha^{2}+\nu^{2} / \alpha-\alpha & =0 \\
\left(\nu^{2} / \alpha^{2}-1\right)(1-\alpha) & =0
\end{aligned}
$$

Solutions:

$(\nu / \alpha)^{2}=1$ or $\alpha=1$

$$
\begin{gathered}
\rightarrow \quad \nu / \alpha=\omega R_{l} C_{l}\left(1+C_{d} / 2 C_{l}\right)= \pm 1 \\
\rightarrow \quad \omega_{c}=\left[R_{l} C_{l}\left(1+\frac{C_{d}}{2 C_{l}}\right)\right]^{-1}
\end{gathered}
$$

The solution $\alpha=1$ results in $C_{d} /\left(2 C_{l}\right)=0$. This is nonphysical, and happens when the capacitance of the liquid is infinitely large, and all the potential falls across the insulating layers in both the conductive and dielectric regime, resulting in a constant electric energy. Getting the factor $\alpha$ closer to 1, however, is important when optimizing the electric energy on the dielectric regime. In other words, increasing $C_{d}$ and decreasing $C_{l}$ will ensure more optimal electrowetting in the dielectric regime. 


\section{Appendix 6B: Electric potential and electric field lines}

In Fig. 6-6 the numerically determined electric energy (and resulting electric force) against droplet position are depicted for drops of varying radius $(R=25,50,100 \mu \mathrm{m})$, and for varying insulator thickness $(d=0.5,2.5,10 \mu \mathrm{m})$ for both the conductive and dielectric regime. For all these parameters the electric potential is plotted in Fig. 6-9. Also equally spaced electric field lines are plotted. The color of the lines indicate the electric field strength $\sqrt{\left(\frac{d U^{2}}{d x}+\frac{d U^{2}}{d y}\right)}$. Fig. 6-8 shows the setup with no drop.

As expected, for the conductive regime the electric field lines do not penetrate the droplet, thus the potential inside the droplet is constant $(0 \mathrm{~V})$. Almost the entire added electric field energy (compared to no droplet present) is located in the insulating layer between the droplet and electrodes (most clearly visible for the $10 \mu \mathrm{m}$ insulator.

For the dielectric cases we do observe a potential drop across the droplet, most notably for the thinnest insulator and largest drop size. In this case the potential distribution and the electric field lines are very similar to the 'no drop'-case in Fig. 6-8, indicating that there is hardly any gain in electric energy caused by the presence of the droplet, and thus that the electrowetting effect will be minimal.

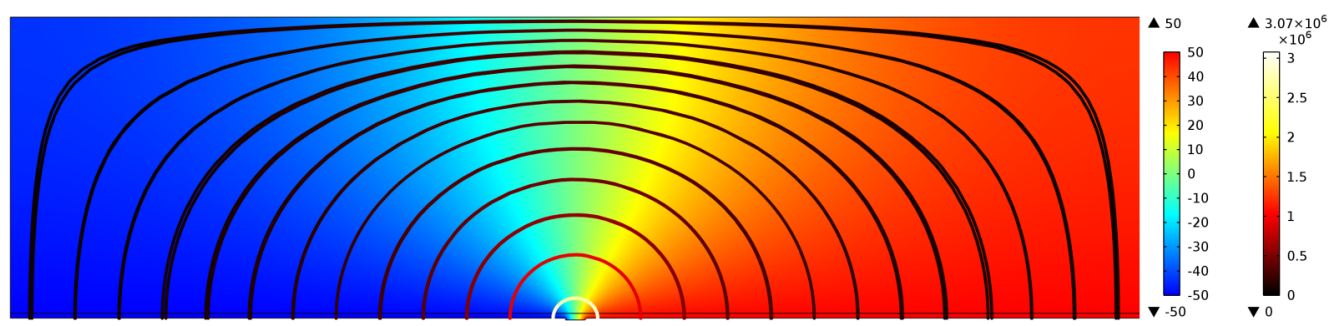

Fig. 6-8 Electric potential and electric field lines if no droplet is present. Electric field lines should form a semicircle from one electrode to the other. A condition that holds in near the gap, but not near the boundaries. 
conductive $\quad \mathrm{d}=0.5 \mu \mathrm{m}$

dielectric
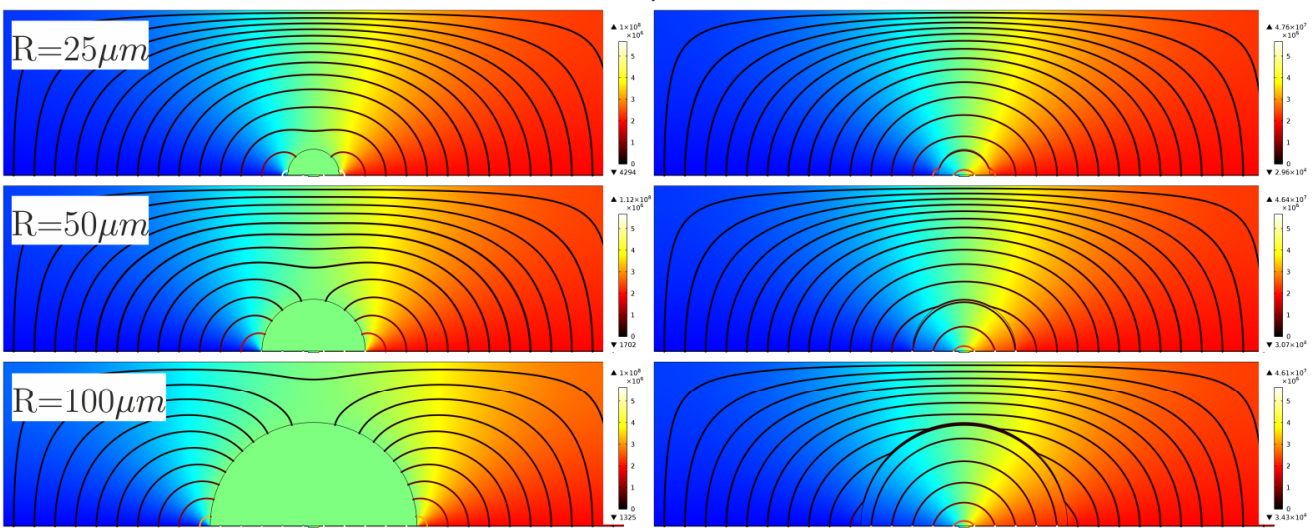

\section{$\mathrm{d}=2.5 \mu \mathrm{m}$}
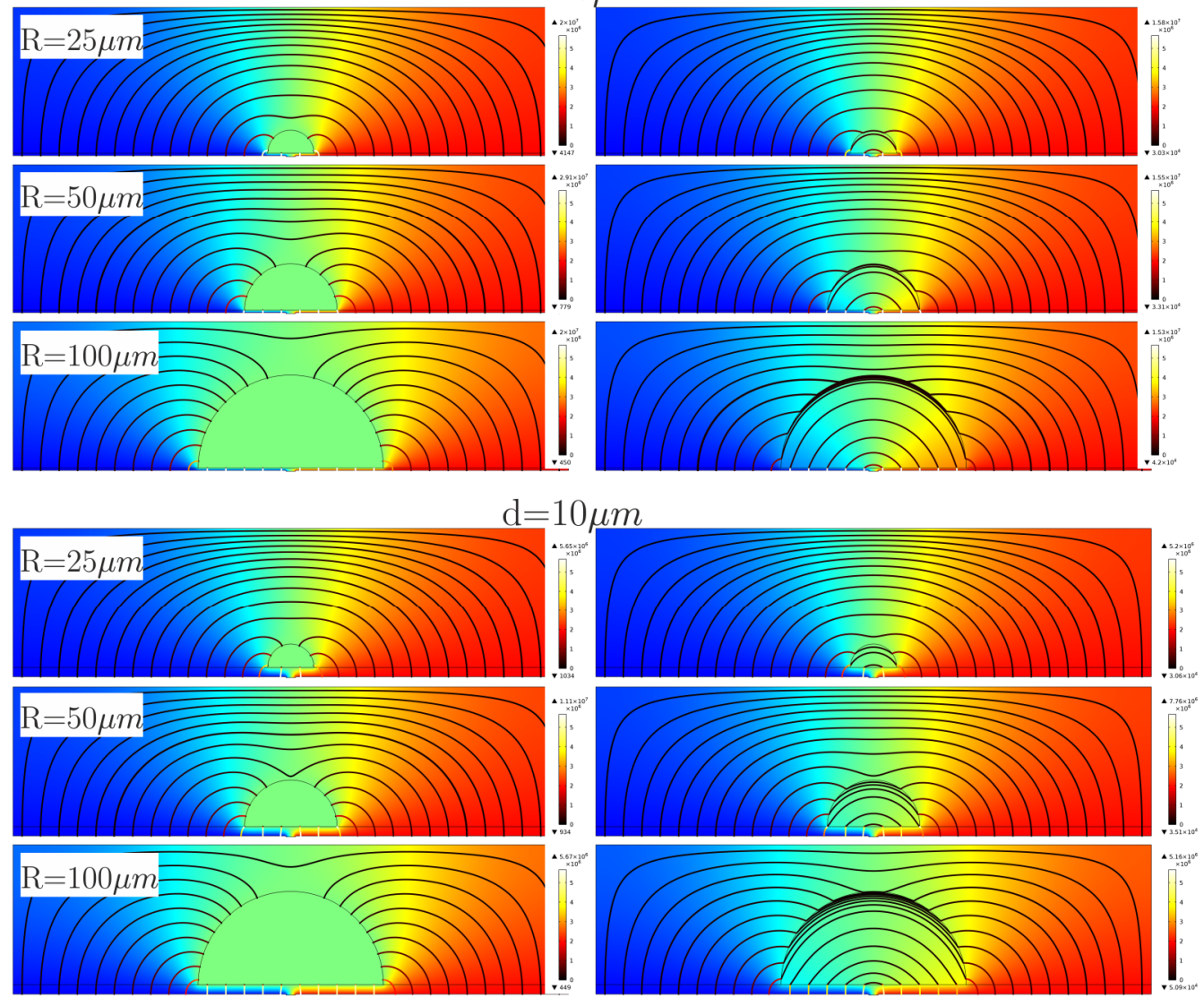

Fig. 6-9 Electric potential and electric field lines for conductive and dielectric drops of 25, 50 and 100 $\mathrm{m}$ atop insulators of 0.5, 2.5 and $10 \mu \mathrm{m}$. 


\section{References}

1. Casadevall i Solvas, X. and A. deMello, Droplet microfluidics: recent developments and future applications. Chemical Communications, 2011. 47(7): p. 1936-42.

2. Seemann, R., M. Brinkmann, T. Pfohl, et al., Droplet based microfluidics. Reports on Progress in Physics, 2012. 75(1).

3. Pit, A.M., M.H.G. Duits, and F. Mugele, Droplet Manipulations in Two Phase Flow Microfluidics. Micromachines, 2015. 6(11): p. 1768-93.

4. Ito, Y., M. Heydari, A. Hashimoto, et al., The movement of a water droplet on a gradient surface prepared by photodegradation. Langmuir, 2007. 23(4): p. 1845-50.

5. Sun, C., X.W. Zhao, Y.H. Han, et al., Control of water droplet motion by alteration of roughness gradient on silicon wafer by laser surface treatment. Thin Solid Films, 2008. 516(12): p. 4059-63.

6. Sun, M., S.S. Bithi, and S.A. Vanapalli, Microfluidic static droplet arrays with tuneable gradients in material composition. Lab on a Chip, 2011. 11(23): p. 3949-52.

7. Dangla, R., S.C. Kayi, and C.N. Baroud, Droplet microfluidics driven by gradients of confinement. Proceedings of the National Academy of Sciences of the United States of America, 2013. 110(3): p. 853-8.

8. Cho, S.K., H.J. Moon, and C.J. Kim, Creating, transporting, cutting, and merging liquid droplets by electrowetting-based actuation for digital microfluidic circuits. Journal of Microelectromechanical Systems, 2003. 12(1): p. 70-80.

9. Malic, L., D. Brassard, T. Veres, et al., Integration and detection of biochemical assays in digital microfluidic LOC devices. Lab on a Chip, 2010. 10(4): p. 418-31.

10. Mugele, F. and J.-C. Baret, Electrowetting: from basics to applications. Journal of Physics: Condensed Matter, 2005. 17(28): p. R705.

11. Jones, T.B., On the relationship of dielectrophoresis and electrowetting. Langmuir, 2002. 18(11): p. 4437-43.

12. Jones, T.B., J.D. Fowler, Y.S. Chang, et al., Frequency-based relationship of electrowetting and dielectrophoretic liquid microactuation. Langmuir, 2003. 19(18): p. 7646-51.

13. Jones, T.B., K.L. Wang, and D.J. Yao, Frequency-dependent electromechanics of aqueous liquids: Electrowetting and dielectrophoresis. Langmuir, 2004. 20(7): p. 2813-8.

14. Hong, J.S., S.H. Ko, K.H. Kang, et al., A numerical investigation on AC electrowetting of a droplet. Microfluidics and Nanofluidics, 2008. 5(2): p. 263-71.

15. Lee, H., S. Yun, S.H. Ko, et al., An electrohydrodynamic flow in ac electrowetting. Biomicrofluidics, 2009. 3(4): p. 44113.

16. Garcia-Sanchez, P., A. Ramos, and F. Mugele, Electrothermally driven flows in ac electrowetting. Phys Rev E Stat Nonlin Soft Matter Phys, 2010. 81(1 Pt 2): p. 015303.

17. Chatterjee, D., B. Hetayothin, A.R. Wheeler, et al., Droplet-based microfluidics with nonaqueous solvents and solutions. Lab on a Chip, 2006. 6(2): p. 199-206.

18. Kumar, A., M. Pluntke, B.J.F. Cross, et al., Charged droplet generation and finite conductivity effects in AC electrowetting. Proc. of the Materials Research Society, 2005.

19. Pit, A.M., R. de Ruiter, A. Kumar, et al., High-throughput sorting of drops in microfluidic chips using electric capacitance. Biomicrofluidics, 2015. 9(4): p. 044116. 
20. 't Mannetje, D., A. Banpurkar, H. Koppelman, et al., Electrically tunable wetting defects characterized by a simple capillary force sensor. Langmuir, 2013. 29(31): p. 9944-9.

21. Jones, T.B., M. Gunji, M. Washizu, et al., Dielectrophoretic liquid actuation and nanodroplet formation. Journal of Applied Physics, 2001. 89(2): p. 1441-8.

22. Geng, H., J. Feng, L.M. Stabryla, et al., Dielectrowetting manipulation for digital microfluidics: creating, transporting, splitting, and merging of droplets. Lab Chip, 2017. 17(6): p. $1060-8$.

23. Verheijen, H.J.J. and M.W.J. Prins, Reversible electrowetting and trapping of charge: Model and experiments. Langmuir, 1999. 15(20): p. 6616-20. 



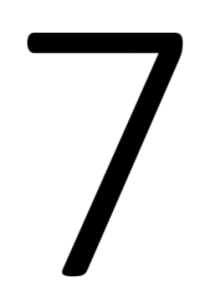

\section{Conclusions and Outlook}

\subsection{Conclusions}

We have developed a tool for the manipulation of tiny water droplets in oil flow. Each required manipulation, i.e. guiding, trapping, release, sorting, merging, and splitting, has been achieved by embedding electrodes in the microchannel walls. Clever branching electrode geometries allowed for the guiding of droplets into parallel streamlines in a wide channel. Zigzagging electrode pairs enabled the trapping of multiple droplets following these different streamlines. Droplets have been sorted at 1200 drops per second. Triggering the sorting electrode to the detected optical signal of fluorescently labeled cells allowed for sorting cell containing droplets at 400 drops per second. Compared to commercial fluorescence assisted cell sorters (FACS) we are still one order of magnitude slower in sorting frequency. We discovered that, because of the relatively small droplet size, even for non-conductive liquids the electric force is large enough for sorting.

Our technique is quite robust, as indicated by the ability to sort droplets for multiple days without detrimental effects. Experimental failures occurred mainly when using very high voltages, resulting in increased wettability of the substrate after a couple of hours. Besides robustness, our technique also excels at strength, accuracy and controllability (by electronics) as compared to other existing techniques.

The choice to use oil flow was dictated by the need for picoliter droplets; a size that cannot be achieved by standard digital microfluidics. As a result of using oil flow there is a strong reduction in the amount of electrodes needed to transport droplets, as compared to DMF. Also much faster movement and higher throughput can be achieved in oil flow. However, the oil flow - and thus droplet transport - is always in one direction, potentially limiting capabilities. Chips need to be leak-tight, and accidents can have expensive consequences. 
While all chips required the use of microfabrication techniques, the most intricate chip design and fabrication can be found in the two-sided chip discussed in Chapter 4. This chip consists of two separate chip halves: one side has insulated electrodes and an open microchannel; the other side includes hydrophilized gold patches enclosed by a hydrophobized glass slide. The gold patches would be required for SPR measurements, but the modular design allows the replacement of this chip half for other detection techniques. Also crucial: the hydrophilic pinning force was strong enough to oppose the drag exerted on the drop, yet the combination of electric and drag force was capable of pulling the droplet from the patch.

Eventhough it is commonly understood that the electrowetting effect extremely diminishes for dielectric drops, our electrostatic potential wells had no difficulty sorting liquids in the dielectric regime. By returning to the theory of electrostatics we explain why our electrostatic potential wells are capable of manipulating dielectric liquids so well. In the end it turns out to be a simple answer: because the droplets in the microchannel are approximately 50 times smaller compared to standard electrowetting setups (while the insulator thickness remains comparable), the contrast in length scales $1 / R$ and $1 / d$ is diminished, while the inherent dielectric contrast between the liquid $(\epsilon \sim 65)$ and insulator $(\epsilon \sim 3)$ remains. This means that the presence of the small droplet allows the build up of a (much) stronger electric field in the system as compared to not having the droplet, which results in a relatively strong electric force.

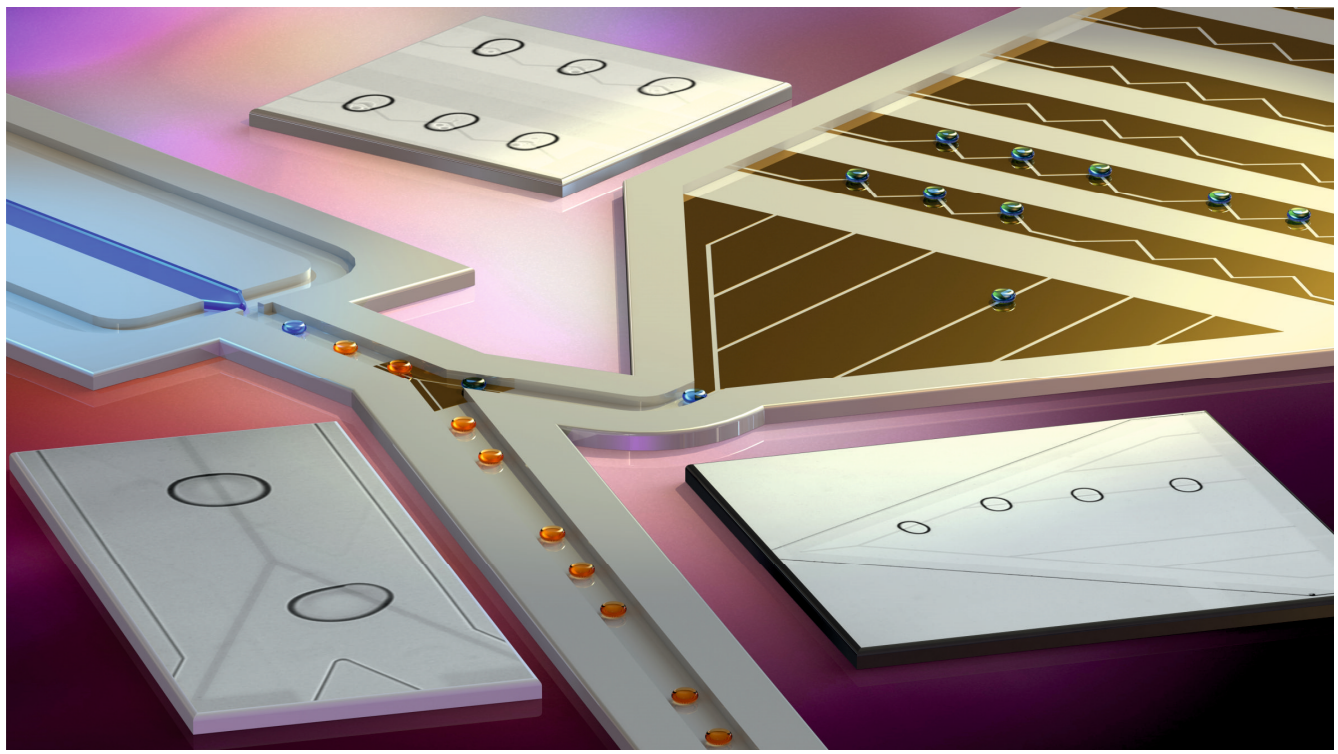

Fig. 7-1 An artist impression of what a microfluidic chip with incorporated droplet generator, droplet sorter, droplet guide and droplet trapping array would look like. 


\subsection{Outlook}

So far, all electric droplet manipulations have been achieved in separate microfluidic chips. Eventually, all droplet manipulations, i.e. droplet generation, sorting, guiding, trapping, and release, will need to be combined with a detection techniques for active cell sorting, e.g. fluorescence, and a diagnostic technique, e.g. SPRi.

Guiding, trapping and release have been combined, but a task still lies in integrating the sorting of droplets. This is not as straightforward as it may seem. Preferentially, one does not wish to drastically alter flow rates while the chip is active. The difference in flow speeds for high speed sorting (milliseconds) and slowly guiding and trapping droplets for analysis (minutes) makes integration difficult. This problem is not insurmountable though, since flowrates can be altered simply by changing relative channel cross sections and the active sorting of droplets can be halted (sending all drops to waste) if all trapping locations are in use.

Another less explicit finding of the thesis work is the discovery that integration of different functionalities can be far from trivial. The project barely got to the stage of studying biological cells inside droplets, partly because of this. The integration of a diagnostics tool like SPRi with the microchip is a task that would require substantial engineering research. An SPR imager is a highly complex system. The use of oil in such complex devices is often undesirable, whereas a spill can cause thousands of euros of damage. Also, the addition of a laser and photomultiplier tube for fluorescence detection, or the addition of electrical wiring, solid state switches and PCBs for electrowetting, makes combining the techniques challenging.

Material properties are another complication when combining different techniques. There is a limited number of materials available to be used as insulator for electrowetting based on requirements for hydrophobicity, a small contact angle hysteresis, inertness, dielectric constant, and ability to form into a homogeneous thin layer. As an example, excellent electrowetting materials like Teflon or parylene-C are difficult to incorporate into fabrication of a leak-tight microfluidic chip. Using fluorinated oils can slowly dissolve fluor-based insulators; silicon oils can dissolve PDMS; in alkane-based oils small hydrocarbons will swell the PDMS, while large hydrocarbons will increase the viscosity and thus the drag force. The tendency of biomolecules, e.g. BSA, to interact with channel walls is another problem that is difficult to resolve. Working in oil, and adding surfactants should make working with biomolecules possible.

As for the viability of electrostatic potential wells in the 'real world', that is a difficult assessment to make. If a technique works with droplets in oil or air, and one surface of the device is available for the placement of electrodes, and droplets do not have to return to the same location twice, and you are capable of microfabrication, then electrostatic potential wells are a great solution. 



\section{Supplemental Movies}

In this thesis several references are made to movies. In the digital version of the manuscript these movies can be viewed by clicking on the images below. The digital version can be downloaded from research.utwente.nl or via DOI: 10.3990/1.9789036544313

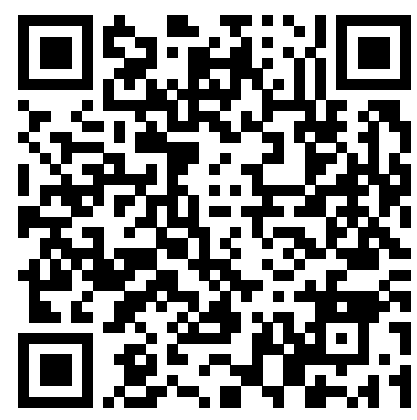

For as long as possible, the movies will also be available on Youtube by following the link in the QR code.

\section{Chapter 1: Introduction}

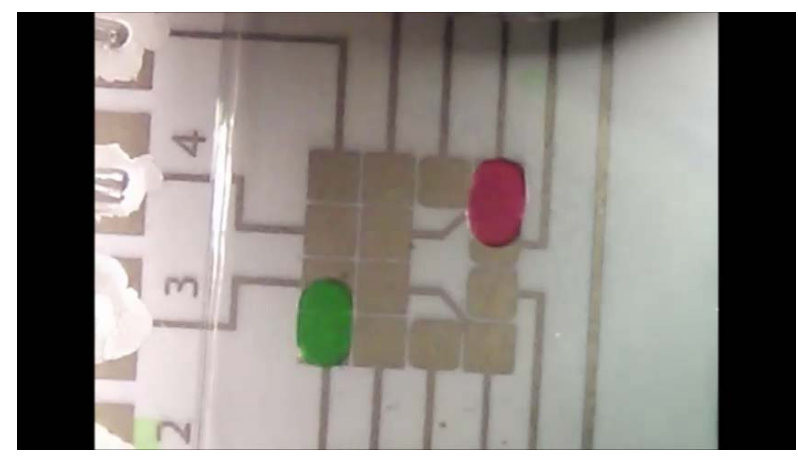

Movie 1-1: Droplet manipulations using the parallel plate electrode geometry as used in Digital Microfluidics. The chip was created during the DigiBio hackathon (December 1213, 2015). Experiment performed in the Physics of complex fluids lab. First simultaneous transport of two droplets is tested. Second, the droplet is split on purpose. Third, we test how fast drops can be transported across the electrodes, and finally we test how fast droplets can be split in two. The bottom substrate is silver ink printed electrodes on photopaper taped to a regular microscope slide. As a dielectric (+- 10um) PTFE foil is used, with a droplet of silicon oil between the film and printed electrodes for better 
connection (no air). On top of the PTFE foil a drop of FC40 is spread for lubrication. The top substrate is an ITO covered microscope slide. This side has no insulation, except for a spincoated layer (50nm) of $0.9 \%$ Cytop solution and baking at 200 degrees Celsius for 1 hour. Droplet: Millipore water $+K C l($ conductivity $=3 \mathrm{mS} / \mathrm{cm})+$ green or red food dye.

\section{Chapter 4: Hydrophilic patch}

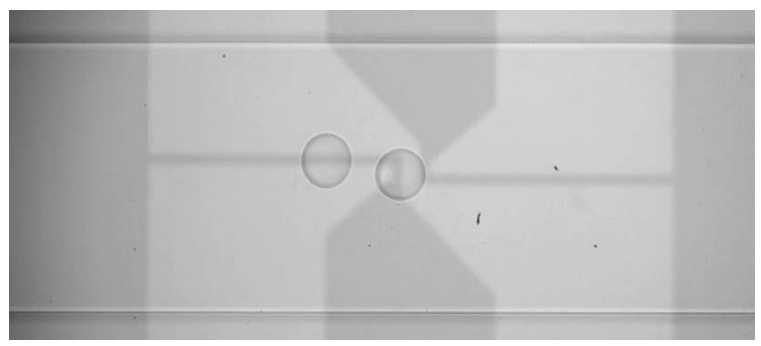

Movie 4-1: The continuous coalescence and release of two droplets at 130V. Movie of the continuous coalescence and release of two droplets at $130 \mathrm{~V}$. Droplets are generated upstream by the flow focusing device. Based on the balance between the drag force (controlled by oil flow rate) and electrostatic force (controlled by the voltage) regimes can be set where below a critical radius a droplet will remain trapped, while above this radius a droplet will release. In this case the critical radius lies between the radius of 1 and 2 coalesced drops. At higher voltages it is also possible to coalesce exactly 3, 4, 5, or 6 droplets. Frame rate: 125 fps; integration time: 1/5000s.

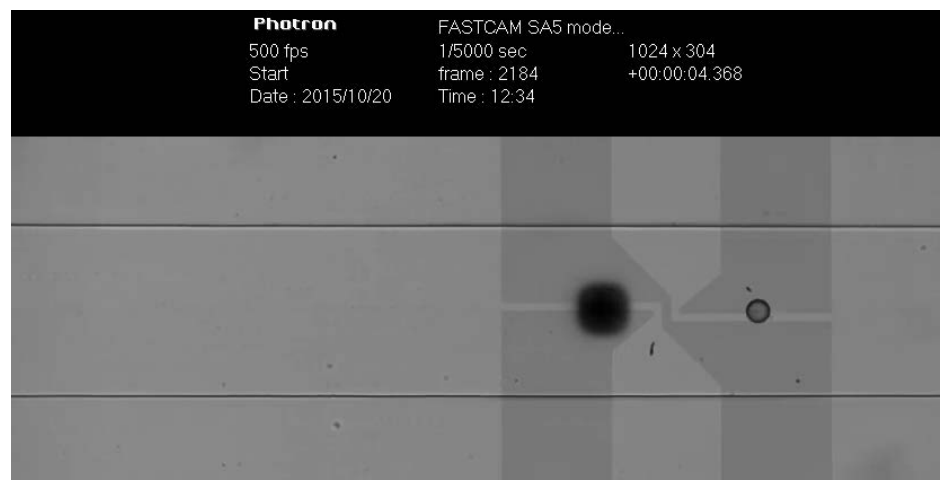

Movie 4-2: Droplets in flow passing and not interacting with the hydrophilic patch. To illustrate the presence of an oil film between the droplet and the hydrophilic patch we show that droplets do not wet the patch in flow. This indicates the need to actively trap a droplet to allow the oil film to break up passively. Frame rate: $125 \mathrm{fps}$; integration time: 1/5000s. 


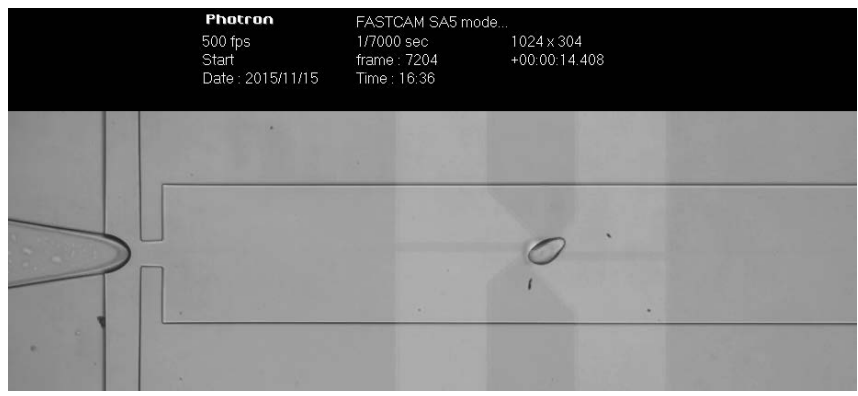

Movie 4-3: Trapping, coalescing, oil film break up, pinning on the hydrophilic patch, and active release by electrowetting. Microscopic movie showing the entire process of 4 droplets being generated on demand (flowrate $285 \mu \mathrm{L} / \mathrm{h}$ ) and merging on the trapping electrodes at a potential of $300 \mathrm{~V}$ at $4 \mathrm{kHz}$. When the trapping electrodes are turned off, the droplet slowly covers the hydrophilic patch indicating that the oil film has indeed been squeezed out between droplet and substrate. After 5.5 seconds the pull off electrodes are activated (300 V, 50Hz, see Snapshot) and the droplet is released from the patch, and flows further down the channel dragged by the oil flow. Notice how afterwards, while flowing in the channel the droplet oscillates at 50Hz, indicating a small Coulomb force pulling on the droplet, which must be caused by a slight charging of the droplet during the trapping or release process. Frame rate: $167 \mathrm{fps}$; integration time: 1/7000 s.

\section{Chapter 5: High speed sorting}

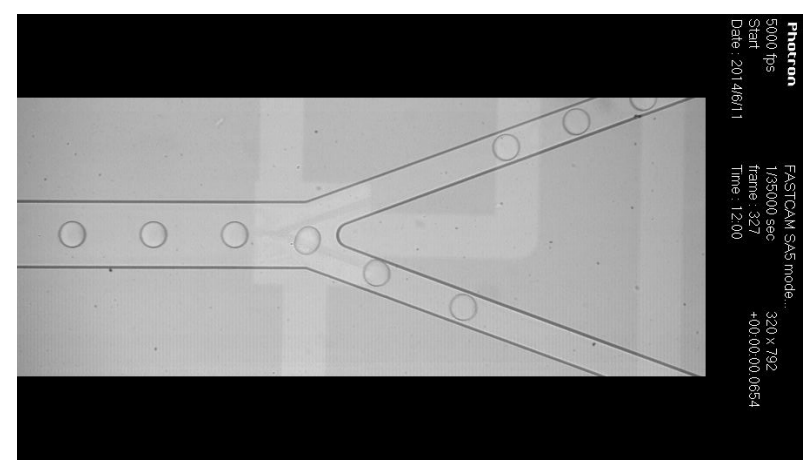

Movie 5-1 for Fig. 5-5: droplet sorting at 1200 drops per second. The sorting of KCl water drops $(R \sim 25 \mu \mathrm{m})$ in FC-40 oil at 1200 drops per second. The sorting electrode is switched on and off every $0.05 \mathrm{~s}$, switching the drops from one outlet to the other. The original movie was 2 seconds (10000 frames 2400 drops), without any drop being sorted in the wrong channel. Movie slowed down 200 times. Channel width: $150 \mu \mathrm{m}$. Recording frame rate: 5000 fps. Recording integration time: 1/35000s. Video frame rate: 25 fps. 


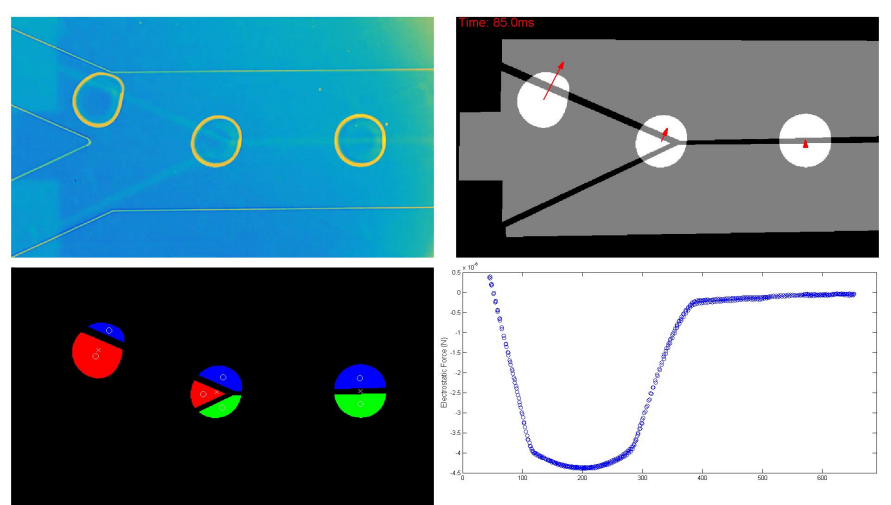

Movie 5-2 for Figure 5-7: analysis of electric force during droplet sorting. Top left: The original movie of $\mathrm{KCl}$ water drops being sorted in light mineral oil at 25 drops per second, recorded at 2000 frames per second. Bottom left: The detection and measurement of the area covered by each drop over each of the three electrodes. Bottom right: live updated plot of the absolute electrostatic force calculated from the measured area ratios. Top right: Overlay plot of the electrode geometry, the detected drop. The red arrows indicate the magnitude and direction of the electrostatic force.

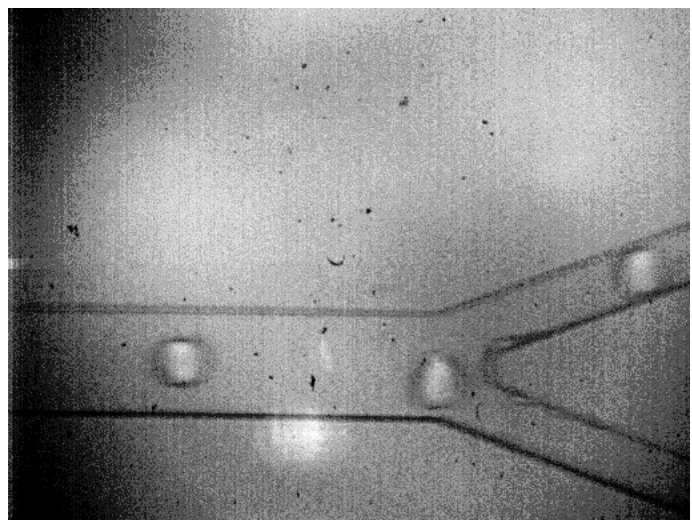

This experiment was performed together with Dr. Aigars Piruska at the PhysicalOrganic Chemistry group at the Radboud University (Netherlands).

Movie 5-3: The sorting of individual drops based on the fluorescence signal detected by the PMTs. Drops are made from a PBS solution containing fluorescently labeled cells, and are generated at 400 drops per second. The PMT detects the fluorescence intensity and the voltage is read in by an FPGA card. When the fluorescence intensity exceeds a pre-set threshold value, the FPGA sends a trigger to the camera. The camera then records 5 frames. At the same time the FPGA sends a $2 \mathrm{~ms}$ voltage signal (10kHz sine, 200 VRMS) to the switching electrode, effectively sorting a single drop containing a fluorescently labeled cell. This movie shows 5 recorded frames, for each trigger signal detected by the PMT. It can be seen that the previous drop is sorted towards the upper channel, and the other (cell-containing) is sorted to the lower channel. Recording frame rate: 2000 fps. 


\section{Chapter 6: Electrowetting in dielectric regime}

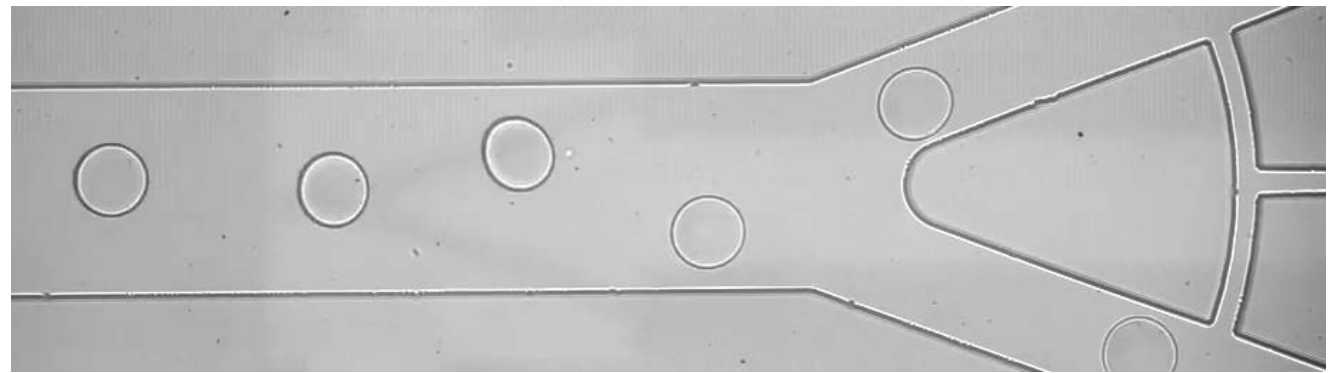

Movie 6-1: the sorting of propylene carbonate (95 V; $50 \mathrm{kHz} ; 50 \mathrm{dps}$ ). Proving that dielectric liquids can be electrically actuated in the microfluidic sorter. 



\section{Summary}

Motivated by the desire to analyze individual cells, the objective of this research is the design, fabrication and implementation of a microfluidic chip capable of manipulating small water droplets in oil flow. In this work droplets are manipulated by electric forces that arise when applying an electric potential over electrodes embedded in the microchannel substrate. Different electrode geometries allow for different actuations. While in oil flow droplets can be guided along a rail, trapped at a specific location, split in two, merged to form larger droplets, and sorted at high speed based on content.

An introduction to electrowetting theory and its applications is given in Chapter 1. To put the technical developments of this thesis in perspective, Chapter 2 contains a review of droplet manipulations in two-phase microfluidics in a broader sense, i.e. using other principles and designs. Passive and active techniques are discussed. Active techniques range from pressure driven to electrical, magnetic, acoustic and optical actuation. The working mechanisms and possibilities of these techniques are explained. The focus lies specifically on the manipulations required for our device: to generate, sort, guide, trap and release the droplets. Furthermore, the different techniques are compared for factors like reliability, force, controllability and cost. According to us the most predictable, controllable and reliable technique capable of performing all the required tasks in a microchannel is the use of electric potential wells.

In chapter 3 the theory and application of electric potential wells is discussed. They are used for the guiding and trapping of droplets in oil flow. Guiding is facilitated by an electrode geometry consisting of multiple electrodes that create different paths for droplets depending on which electrodes are actuated. In this case the electrodes branch into 6 paths which laterally guide droplets to 6 lanes in the microchannel. Consecutively, 36 guided droplets are trapped against the oil flow at arrays of trapping electrodes. Other actuations accomplished in this chapter are the splitting and sorting of droplets.

Chapter 4 is an extension of the capability to trap and release droplets. When using SPR as a diagnostics tool, the contents of a droplet will need to interact with antibodies printed on the substrate. These patches of antibodies will form hydrophilic areas in an otherwise hydrophobic channel. We develop a fabrication process to create a dual-sided microchip with actuation electrodes in the bottom substrate, and hydrophilic gold patches on the hydrophobic top substrate. We focus on the trapping and release of droplets in the presence of a hydrophilic patch. Eventually we were capable of electrically trapping a droplet long enough for the oil film between the drop and the patch to break up. The droplet then sticks to the hydrophilic patch in oil flow. By using a secondary pair of electrodes the droplet can be pulled from the patch. 
In chapter 5 we optimize the system for sorting droplets as described in chapter 3 to enable the sorting of droplets at high speeds. The working principle of our sorter is based on a contrast in conductivity between the drop and the continuous ambient phase, which ensures successful operation even for drops of highly conductive biological media like phosphate buffered saline. Moreover, since the electric field does not penetrate the drop, its content is protected from electrical currents and Joule heating. A simple capacitive model allows quantitative prediction of the electrostatic forces exerted on drops. The maximum achievable sorting rate is determined by a competition between electrostatic and hydrodynamic forces. Sorting speeds up to 1200 per second are demonstrated for conductive drops of $160 \mathrm{pL}$ in low viscosity oil. Finally, together with the Radboud university, experiments are performed to sort droplets containing fluorescently labeled cells.

In traditional electrowetting setups the electric forces strongly decrease when going to high AC frequencies. Our method used for manipulating droplets electrically turns out to be - not only very versatile, but also - applicable even beyond the usual AC frequencies for electrowetting, as evidenced by successful high speed sorting experiments using liquids in this so-called 'dielectric regime'. Chapter 6 is a more theoretical chapter on electrowetting and the difference in actuating at different frequencies where a drop can be considered conductive or a dielectric. By going back to the basics of electrostatics the parameters that dictate the magnitude of electric energy and electric force are described. It turns out that the decrease in electric energy in the dielectric regime lies in relative geometric length scales of the droplet and insulating layer and the dielectric contrast between these two materials.

Ultimately, we demonstrated a novel combination of techniques for aqueous droplet manipulations in two phase microfluidics. The control by electronics makes our system very accurate and manageable. The relatively large and predictable forces acting on the droplet are advantageous, but the complex fabrication method are costly. Whether the downsides outweigh the advantages for practical applications remains to be seen. 


\section{Samenvatting}

Gemotiveerd door het verlangen om individuele cellen te analyseren, is het doel van dit onderzoek een microfluïdische chip te ontwerpen, fabriceren en implementeren, die kleine waterdruppels in stromende olie kan manipuleren. In dit werk worden druppels gemanipuleerd door elektrische krachten, die ontstaan door het aanbrengen van een elektrische potentiaal tussen elektroden, die in de bodem van het microkanaal zijn ingebouwd. Verschillende elektrodegeometrieën zorgen voor verschillende acties. Terwijl de druppels in de olie stromen kunnen ze langs een spoor worden gestuurd, of vast worden gehouden op een specifieke locatie, of in tweeën gesplitst, of samengevoegd tot grotere druppels, of op hoge snelheid gesorteerd op basis van hun inhoud.

In hoofdstuk 1 wordt de theorie van electrowetting en de toepassingen ervan beschreven. Om de technische ontwikkelingen van dit proefschrift in perspectief te plaatsen, bevat hoofdstuk 2 een overzicht van druppelmanipulaties in tweefase microfluidica in bredere zin, d.w.z. met gebruik van andere technieken. Passieve en actieve technieken worden besproken. Actieve technieken variëren van op druk gebaseerde naar elektrische, magnetische, akoestische en optische besturing. De werkmechanismen en mogelijkheden van deze technieken worden uitgelegd. De nadruk ligt specifiek op de benodigde manipulaties voor ons apparaat: het genereren, sorteren, begeleiden, vasthouden en loslaten van de druppels. Bovendien worden de verschillende technieken vergeleken met betrekking tot factoren zoals betrouwbaarheid, kracht, controleerbaarheid en onkosten. Volgens ons is de meest voorspelbare, regelbare en betrouwbare techniek, die alle vereiste taken in een microkanaal kan uitvoeren, het gebruik van elektrische potentiaalputten.

In hoofdstuk 3 wordt de theorie en toepassing van elektrische potentiaalputten besproken. Ze worden gebruikt voor het sturen en vasthouden van druppels in stromende olie. Sturen wordt gedaan door een elektrodegeometrie bestaande uit meerdere elektroden, die verschillende paden voor druppels creëren, afhankelijk van welke elektroden worden geactiveerd. In dit geval vertakken de elektroden in 6 paden, die lateraal druppels leiden naar 6 banen in de microkanaal. Opeenvolgend worden 36 gestuurde druppels vastgehouden tegen de oliestroom door een reeks van vasthoud-elektroden. Andere handelingen die worden verwezenlijkt zijn het splitsen en sorteren van druppels.

Hoofdstuk 4 is een uitbreiding van de mogelijkheid om druppels vast te houden en weer los te laten. Bij gebruik van SPR als diagnostisch hulpmiddel, moet de inhoud van een druppel interactie aangaan met antilichamen, die op het substraat zijn geprint. Deze spots met antilichamen zullen hydrofiele gebieden vormen in een voor de rest hydrofoob kanaal. We ontwikkelen een fabricageproces om een dubbelzijdige microchip te creëren met manipulatie-elektroden in de bodem en hydrofiele gouden spots op de hydrofobe 
bovenkant. Met deze chip richten we ons op het vasthouden en loslaten van druppels in aanwezigheid van een hydrofiele spot. Uiteindelijk waren we in staat om een druppel lang genoeg elektrisch vast te houden, zodat de olielaag tussen de druppel en de spot op kan breken. De druppel blijft vervolgens - ondanks de stromende olie - vastplakken aan de hydrofiele spot. Door gebruik te maken van een secundair paar elektroden kan de druppel van de hydrofiele spot worden losgetrokken.

In hoofdstuk 5 optimaliseren we het sorteersysteem, zoals beschreven in hoofdstuk 3, om de druppels op hoge snelheid te sorteren. Het werkingsprincipe van onze sorteerder is gebaseerd op een contrast in de conductiviteit tussen de druppel en de olie. Hierdoor is een succesvolle werking garandeert, zelfs voor druppels van zeer geleidende biologische media, zoals een fysiologische zoutoplossingen. Aangezien het elektrische veld niet in de druppel doordringt is de inhoud ervan beschermd tegen elektrische stromen en opwarming. Een simpel capacitief model maakt het mogelijk om een kwantitatieve voorspelling van de elektrostatische kracht op de druppels te geven. De maximaal haalbare sorteersnelheid wordt bepaald door de competitie tussen elektrostatische en hydrodynamische krachten. Sorteersnelheden tot 1200 per seconde zijn behaald voor geleidende druppels van $160 \mathrm{pL}$ door gebruik te maken van olie met een lage viscositeit. Ook hebben we samen met de Radboud universiteit sorteerexperimenten uitgevoerd met druppels, die fluorescent gelabelde cellen bevatten.

Bij traditionele electrowettingexperimenten neemt de elektrische kracht sterk af bij hoge frequenties van de wisselstroom. Onze methode blijkt niet alleen zeer veelzijdig, maar ook bruikbaar te zijn buiten de gebruikelijke wisselstroom frequenties. Dit blijkt uit succesvolle sorteerexperimenten met druppels in dit zogenaamde 'diëlektrische regiem'. Hoofdstuk 6 is een meer theoretisch hoofdstuk over electrowetting en het gebruik van verschillende frequenties, waarbij een druppel de ene keer geleidend dan wel diëlektrisch kan worden beschouwd. Door terug te gaan naar de basis van elektrostatica worden de parameters die de grootte van elektrische energie en elektrische kracht dicteren beschreven. Het blijkt dat de daling van de elektrische energie in het diëlektrische regime ligt in relatieve geometrische lengteschalen van de druppel en de isolerende laag en het diëlektrische contrast tussen deze materialen.

Uiteindelijk hebben we door middel van een nieuwe combinatie van technieken waterdruppels in olie gemanipuleerd. De controle door middel van elektronica maakt ons systeem zeer accuraat en beheersbaar. De relatief grote - en voorspelbare krachten, die op de druppel werken, zijn gunstig, maar de complexe fabricagemethode maakt ons systeem prijzig. Of de nadelen groter zijn dan de voordelen voor praktische toepassingen, valt nog te bezien. 


\section{List of publications}

\section{Scientific journals}

Trapping of drops by wetting defects

D.J.C.M. 't Mannetje, S. Ghosh, R. Lagraauw, S. Otten, A.M. Pit, C. Berendsen, J. Zeegers, D. van den Ende, and F. Mugele

Nature Communications, 5, 3559 (2014)

Electrostatic potential wells for on-demand drop manipulation in microchannels

R. de Ruiter, A.M. Pit, V.M. de Oliveira, M.H.G. Duits, D. van den Ende, and F. Mugele Lab on a Chip 14 (5), 883-891 (2014)

High-throughput sorting of drops in microfluidic chips using electric capacitance A.M. Pit, R. de Ruiter, A. Kumar, D. Wijnperlé, M.H.G. Duits, and F. Mugele, Biomicrofluidics 9 (4), 044116, (2015)

Droplet manipulations in two phase flow microfluidics

A.M. Pit, M.H.G. Duits, and F. Mugele

Micromachines 6 (11), 1768-1793 (2015)

Electrode-assisted trapping and release of droplets on hydrophilic patches in a hydrophobic microchannel

A.M. Pit, S. Bonestroo, D. Wijnperlé, M.H.G. Duits, and F. Mugele

Microfluidics and Nanofluidics 20 (9), 123 (2016)

Jumping drops on hydrophobic surfaces, controlling energy transfer by timed electric actuation

Z. Wang, D. van den Ende, A.M. Pit, R. Lagraauw, D. Wijnperlé, F. Mugele

Soft Matter 13, 4856-4863 (2017)

Electrowetting in the dielectric regime

A.M. Pit, A. Cavalli, D. van den Ende, M.H.G. Duits and F. Mugele

Article in preparation (2017) 


\section{Conference contributions}

\section{Presentations}

- International Meeting on Electrowetting (2014), Cincinnati

Electrostatic drop control in microfluidics

- Physics@FOM (2016), Veldhoven

Electrostatic droplet manipulations in two-phase flow microfluidics

- JMBC Burgersdag, (2016), Lunteren

Electrostatic droplet manipulations in two-phase flow microfluidics

- Soft matter+ day (2017), Enschede

Electrowetting: How it works, $\mathcal{E}$ what it is used for

\section{Posters}

- MESA+ day (2012), Enschede

- My drop will (not) go on: trapping in electrically tunable potential wells $\delta$

D.J.C.M. 't Mannetje, A.M. Pit, R. de Ruiter, R. Lagraauw, H.T.M. van den Ende, F. Mugele

$1^{\text {st }}$ prize winner MESA+ day

- STW Jaarcongres (2013), Utrecht

CellSPRead: Paving the way towards personalized cancer therapy

I. Stojanovic, A.M. Pit, R.B.M. Schasfoort, M.H.G. Duits, F. Mugele, and L.W.M.M.

Terstappen

$1^{\text {st }}$ prize winner of the Simon Stevin Leerling-prijs

- MicroTAS (2013), Freiburg

Electrostatic potential wells for manipulations of drops in microchannels

R. de Ruiter, A.M. Pit, V. Martins de Oliveira, D. Wijnperlé, M.H.G. Duits, H.T.M. van den Ende, and F. Mugele

- FLOW14 (2014), Enschede

- Physics@FOM (2013, 2014), Veldhoven

- JMBC Burgersdag (2015), Lunteren 


\section{Acknowledgements}

Perhaps the best and worst part about writing a thesis: the acknowledgements. It means that I am close to finish. It means that I have to look back on my successes and failures over the past years. It is the one part of the thesis that people will actually read. There is a fine line to tread between honesty and ego stroking. And worst of all: what if I forget to mention someone? I have to come across as a well-rounded individual by making semifunny, or semi-wise statements. With that in mind, I think writing acknowledgements is a conspiracy towards new PhD students: we are pretending that doing a PhD is not essentially a solo endeavor.

I would like to thank ...

... my favorite professor Frieder Mugele for giving me the chance to do my $\mathrm{PhD}$ at the Physics of Complex Fluids group. The research in your group touches such a wide range of topics that there is a steep learning curve for any new person joining the group. It was a pleasure to learn about each and every subject, and I am sure this variety of topics will help me in the future. You are always honest with your judgement, which is refreshing in a society where feelings increasingly trump rationality. I fondly remember the $\mathrm{PhD}$ dinner at your home, even though I did have to very subtly invite myself. Thank you for letting me enjoy your group for an extended period. Your support helped me and motivated me to finish.

... my favorite supervisor Michèl Duits for his guidance. You have been indispensable, especially when writing publications and this thesis. Whenever I send you a piece of text, you have comments available within no-time, with subtle changes that make the story flow more naturally. Our complementary dispositions make working together productive. Where I focus on details, you keep the overview, and where I improvise, you force me to make a planning - with varying rates of success. When I feel uninspired, you give an extra push $\vec{J}=\vec{F} \cdot \Delta t$. Thank you.

... all the opponents of the promotion committee - Leon Terstappen, Mireille Claessens, Jan Eijkel, Jaap den Toonder, and Patrick Onck - who were willing to take the time to read my thesis and think of appropriate questions. I do hope you appreciate my efforts.

... my favorite Dirk van den Ende. I always admire the way you combine intellect and social skills. How you manage to explain physical problems by understanding the level of 
the listener. Also the occasional 'well-deserved' stomp was a pleasure to receive.

... all my favorite secretaries: Annelies Cohn-Guichelaar, Lisette Hampsink, Edith van Eijk, Isabel Benes-Gollenbeek. It goes without saying that without your contributions nothing would be achieved in the group whatsoever.

... all my favorite current colleagues: Alessandro Beltram, Aram Klaassen, Beybin Ilhan, Carla Annink, Chamy Mahendran, Davood Baratian, Daniel Wijnperlé, Igor Sîretanu, Jun (Ju-in) Gao, Martin Haagh, Nathalie Schilderink, Olena Kudina, Simone Rinie van Lin, Stelian Pintea, and Qierui Zhang.

... all my favorite past colleagues: Agata Brzozowska, Alberto Gomez-Casado, Andrea Cavalli, Armando Maestro-Martin, Burak Eral, Cunlu Zhao, Daniel Ebeling, Daniel Hagedoorn, Dieter 't Mannetje, Duc Nguyen, Edoardo Mazzi, Fei Liu, Ivan Stojanovic, Ivo Roghair, Jolet de Ruiter, Jorick van 't Oever, Jung Min Oh, Lei Wang, Mariska Grevelink, Martien Cohen-Stuart, Nicolao Cerquiera Lima, Riëlle de Ruiter, Sissi de Beer, Yan Zhang, and Zhantao Wang.

... all my favourite Indians: Aditya Narayanan, Arun Banpurkar, Arun Gnanappa, Bijoy Bera, Chandra Murade, Dileep Mampallil, Dirhendra Tiwari, Kartikeya Mishra, Naveen Kumar, Omkar Deshmukh, Ranabir Dey, Sachin Nair, and Somnath Ghosh.

... all my favorite bachelor and master students whom I annoyed tremendously helped occasionally: Arne te Nijenhuis, Harmen Hoek, Mathijs van Gorcum, Rudy Lagraauw, Simon Otten, Vitor Martins de Oliveira. Special thanks go to Sander Bonestroo who worked his ass off in the lab to get that final successful experiment running, resulting in the publication of Chapter 4. And special thanks to Anand Kumar for contributing to the high speed sorting experiments.

All these people have made my stay at PCF memorable, pleasurable, interesting, successful, and/or entertaining. For instance, my two paranymphs (and former office mates) Aram and Martin. When I first met Aram I thought he was an arrogant little bugger. Since then my opinion has not altered. However, I cannot deny that Aram is always helpful to people in need, loves to play devil's advocate in a discussion, and has your back in difficult situations. Martin is a food technician and therefore knows everything. Besides being down to earth, he is a social critter that always regales us with stories of his weekend adventures. Thank you both for willing to take the heavy burden of paranymphhood.

When mentioning Martin, Simone is just a keystroke on Skype away. Her omnipresent laugh brightens the Meander. Simone and Aditya love to organize group outings and 'borrels' together. Those days often end at Happy Italy or the Beiaard, together with regulars Sachin, Qierui, Aram, Martin, Beybin, and Alessandro, or irregularly supplemented by Carla, Michèl, Davood and Chamy. And let's not forget the movie nights at Aram's or my house, a PubQuiz, burgers at the Haagh's and Indian BBQ at Aditya's. 
I also wish to thank my former office mates from the numerical inclination: Ivo, Andrea and Nicolao. Your presence brought warmth to my heart, most likely caused by the incessant rattling of that monstrous computer. Thanks to you I realize that numerical simulations are more than just copying formulas from other people's work and looking out of the window with your legs raised up while the computer does all the work.

I cannot forget the fun times we had on game nights with Riëlle, Jolet, Dieter, Andrea and he who must not be named. Also, Riëlle, thank you for the cooperation we had while publishing our Lab on a Chip paper. And Andrea for the discussions and explanations of numerical calculations for electrowetting. I surely learned a lot about publishing an article from both of you.

I speak my appreciation for all the people who have taken the red pill, all the Joylent aficionados and all the highly skilled Frisbee players that made the lunch breaks more active. I firmly believe that a bit of exercise during a mostly sedentary existence is a necessity.

Lastly, I would like to thank my parents for raising me in a safe and free environment. Without me noticing, they instilled in me the desire for education and the ability of logical thinking. They sheltered me from the notion of the existence of an omnipresent, omnipotent, omniscient being. One of my oldest memories pertains standing outside our house in Belgium on a bright day, my father holding an apple and an orange, mimicking the path the earth travels around the sun. And - while pointing at the sun - stating that, if the sun exploded now, it would take the light 8 minutes to travel from the sun towards earth. As this story shows, as a child I was not confronted with the harshness of the outside world, but also not shielded from acknowledging its existence. 



\section{About the author}

Arjen Michiel Pit was born the $18^{\text {th }}$ of April 1983 in the hospital of Drachten, the Netherlands to his father Jes Pit and mother Ida Pit-Galama. Together with his brothers Jurjen and Marcel he lived his formative years in Zedelgem, Belgium. At the age of 8 the family moved to the rustic village of Epe, Netherlands, where Arjen attended the RSGNOV from which he obtained his $\mathrm{VWO}^{+}$degree.

In 2007 he acquired his bachelor's degree in Biomedical Engineering at the University of Twente. His final bachelor assignment was performed at the BioPhysical Engineering group of Vinod Subramaniam. His work involved assisting in the development of the StarCount system: a device for low-cost point-of-care determination of the white blood cell count of HIV patients from a pinprick of their whole blood.

In 2011 he received his master's degree in Biomedical Engineering at the University of Twente. His final Masters' assignment was executed at the Medical Cell BioPhysics group of Leon Terstappen. Under the supervision of Cees Otto his work concerned the enhancement of the Raman setup to enable $4 \mathrm{D}$ analysis. This resulted in $3 \mathrm{D}$ visualization of the distribution of DNA (nucleus and nucleoli), lipid vesicles, the cell membrane and the presence of gold nanorods inside SKBR3 cancer cells.

He then started his $\mathrm{PhD}$ research at the Physics of Complex Fluids group within the MESA+ Institute for Nanotechnology and the Department of Science and Technology of the University of Twente under supervision of prof. Frieder Mugele and Dr. Michèl Duits. The research entailed the manipulation of water droplets in microfluidic channels by use of electrodes, the results are published in this thesis.

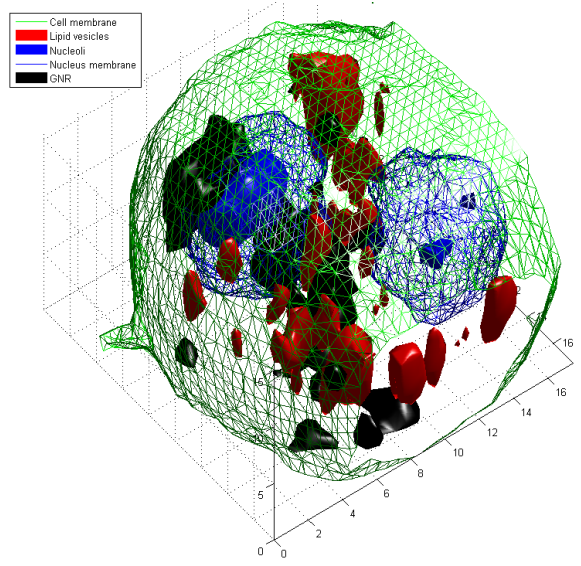

$3 D$ representation of an immobilized SKBR3 cancer cell, showing distribution of the lipid vesicles (red), cell nucleus (blue, wire frame) and nucleoli (blue), cell membrane (green wire frame) and gold nanorods (black) absorbed by the cell.

Molecular specificity determined from specific peaks of the a Raman spectral scan at each $x, y, z$ location. 